\title{
SAMPLE DATA FOR THE GEOLOGIC MAP OF THE ISLAND OF HAWAII
}

\author{
Compiled by Edward W. Wolfe and Jean Morris
}

These maps and tables give locations, results, and documentary data for 1,783 analyzed rocks (table 1) and 242 radiocarbon ages (table 2). These data support the Geologic Map of the Island of Hawaii (Map I-2524-A), which provides descriptions of all map units and geologic context for the samples. Most samples were collected during the course of the geologic mapping, and all were related to specific geologic map units.

Samples are listed by $71 / 2$-minute topographic quadrangle, and each quadrangle is represented by a two-letter code (fig. 1). Within each quadrangle, analyzed rock samples are numbered in sequence from south to north, beginning with 1 (for example, BO-
1). Gaps in numbering represent analyzed samples that were culled from the set either because of poor quality, indicated by excessive analyzed $\mathrm{H}_{2} \mathrm{O}$ or loss on ignition, or redundancy.

Some radiocarbon ages are irreconcilable with well established stratigraphic constraints and have been omitted from the data set. Such ages are generally too young and are commonly determined for charcoal collected at the bases of aa flows. Repeated experience indicates that charcoal fragments (for example, from forest fires long after a lava flow was emplaced) readily sift through and contaminate any charcoal that might have been preserved when the flow was emplaced.

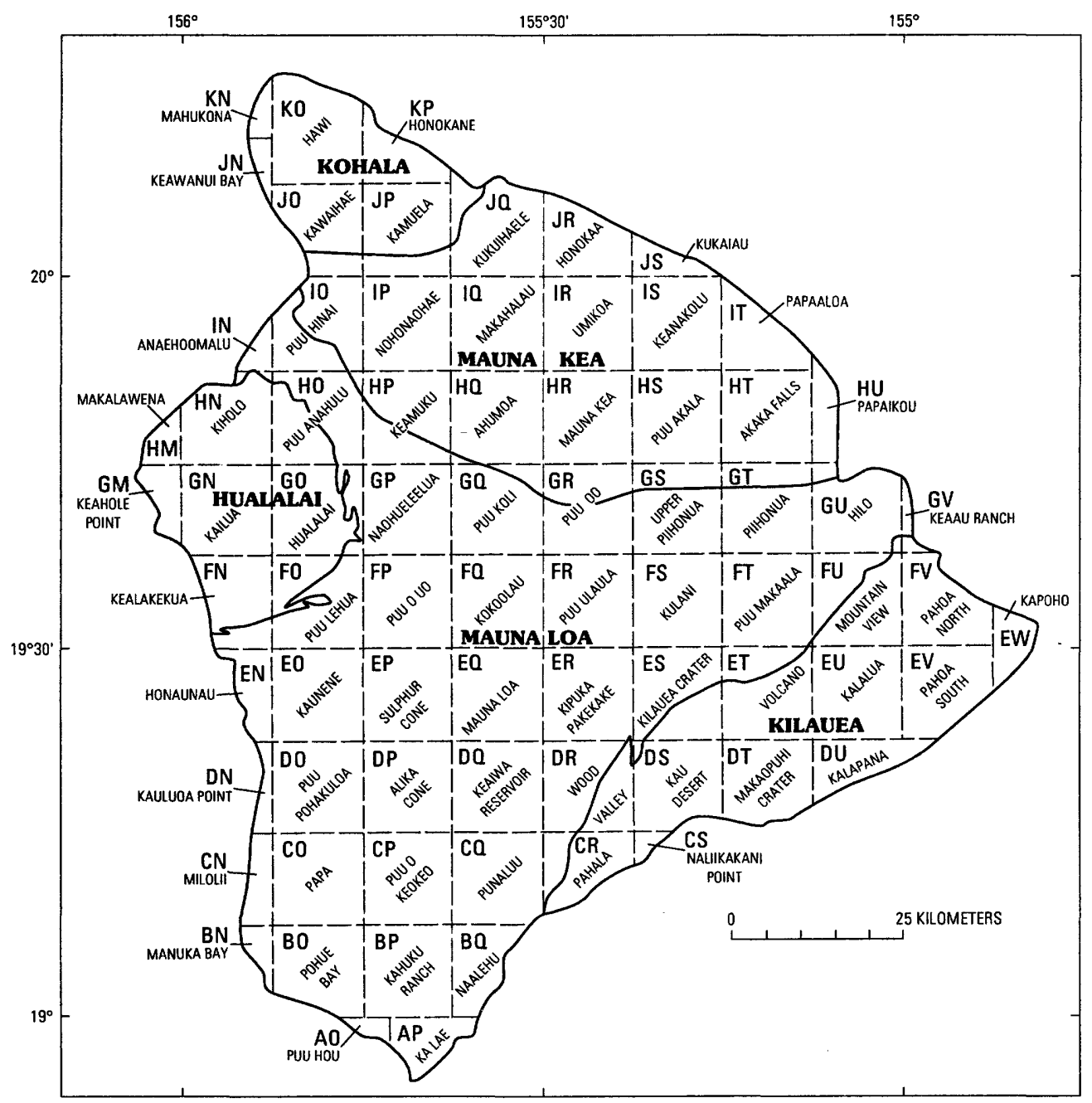

Figure 1. Index map identifying 71/2-minute quadrangles, quadrangle codes, and volcano boundaries. 
Table 1.-Chemical analyses

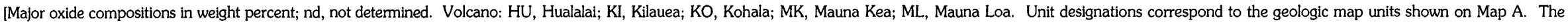

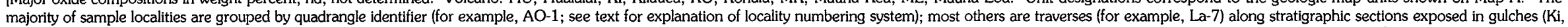



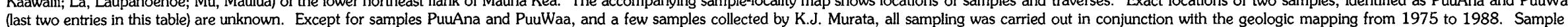

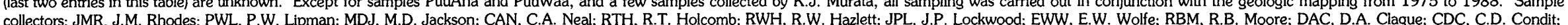
FAT, F.A. Trusdell; JBB, J.M. Buchanan-Banks; WSW, W.S. Wise; MAK, M.A. Kuntz; MOG, M.O. Garcia; SCP, S.C. Porter; KJM, K.J. Murata.

Remarks: a X-ray fluorescence analyses; analyst, J.M. Rhodes, University of Massachusetts, Amherst, Mass.

b X-ray fluorescence analyses; $\mathrm{H}_{2} \mathrm{O}$ specifically determined only for those samples analyzed for $\mathrm{CO}_{2}$; otherwise, $\mathrm{H}_{2} \mathrm{O}$ content is loss on ignition. Analysts: A.J. Bartel, E. Robb, D. Siems, K. Stewart, J. Taggart, U.S. Geological Survey, Denver, Colo.; J. Evans, R. Johnson, Reston, Va.

c Rapid-rock analyses using single-solution procedure of Shapiro (1975). Analysts: J. Gillison, Z. Hamlin, M. Kavulak, D. Kay, D. Kobilis, L. Mei, H. Smith, R. Somers, U.S. Geological Survey, Reston, Va. X-ray fluorescence analyses; analyst, F.A. Frey, Massachusetts Institute of Technology, Cambridge, Mass.

e Classical chemical analyses; analysts: P. Buschman, E.S. Daniels, E.L. Munson, D.F. Powers, R.T. Okamura, U.S. Geological Survey, Denver, Colo. Collected by K.J. Murata (unpub. data, 1962-1966). Lava flow of Waawaa Trachyte Member of the Hualalal Volcanics, collected from Pun An of

(Cross, 1904). The data of this table are available as a digital electronic file (Wolfe, 1996)

\begin{tabular}{|c|c|c|c|c|c|c|c|c|c|c|c|c|c|c|c|c|c|c|c|c|}
\hline $\begin{array}{l}\text { Sample } \\
\text { locality } \\
\text { No. }\end{array}$ & $\begin{array}{l}\text { Vol- } \\
\text { cano }\end{array}$ & Unit & $\begin{array}{l}\text { Map } \\
\text { sheet } \\
\text { No. }\end{array}$ & $\begin{array}{l}\text { Laboratory } \\
\text { No. }\end{array}$ & Remark & Collector & $\mathrm{SiO}_{2}$ & $\mathrm{Al}_{2} \mathrm{O}_{3}$ & $\mathrm{Fe}_{2} \mathrm{O}_{3}$ & $\mathrm{FeO}$ & $\mathrm{MgO}$ & $\mathrm{CaO}$ & $\mathrm{Na}_{2} \mathrm{O}$ & $\mathrm{K}_{2} \mathrm{O}$ & $\mathrm{H}_{2} \mathrm{O}$ & $\mathrm{TiO}_{2}$ & $\mathrm{P}_{2} \mathrm{O}_{5}$ & $\mathrm{MnO}$ & $\mathrm{CO}_{2}$ & Total \\
\hline $\mathrm{AO}-1$ & $\mathrm{ML}$ & k5-1868 & 3 & ML-84 & $a$ & JMR & 46.90 & 8.29 & 12.57 & nd & 22.21 & 6.58 & 1.16 & .25 & nd & 1.30 & .15 & .16 & nd & 99.57 \\
\hline AO-2 & ML & k5-1868 & 3 & ML-83 & a & JMR & 50.97 & 12.71 & 12.08 & nd & 9.18 & 9.96 & 2.09 & .40 & nd & 2.04 & .24 & .16 & nd & 99.83 \\
\hline AP-1 & ML & k1o & 3 & SW-29 & a & JMR & 50.95 & 13.32 & 1.83 & 9.04 & 8.38 & 11.10 & 2.18 & .38 & nd & 1.87 & .23 & .17 & nd & 99.45 \\
\hline AP-2 & ML & kh & 3 & SW-43 & a & PWL & 50.87 & 13.93 & 12.67 & nd & 6.60 & 10.72 & 1.91 & .22 & nd & 2.35 & .24 & .18 & nd & 99.69 \\
\hline AP-3 & ML & kh & 3 & sw-42 & a & PWL & 50.23 & 11.97 & 11.74 & nd & 12.75 & 9.30 & 2.70 & .25 & nd & 1.78 & .19 & .17 & nd & 101.08 \\
\hline AP-4 & ML & kh & 3 & SW-40 & $\mathrm{a}$ & PWL & 45.71 & 7.11 & 12.27 & nd & 25.72 & 5.71 & 1.45 & .15 & nd & 1.06 & .11 & .17 & nd & 99.46 \\
\hline BO-1 & $\overline{M L}$ & k3 & 3 & SW-25 & a & JMR & 51.58 & 13.53 & 2.30 & 8.64 & 7.59 & 10.26 & 1.93 & .45 & nd & 2.07 & .26 & .16 & nd & 98.77 \\
\hline BO-2 & ML & $k 3$ & 3 & SW-6 & $\mathrm{a}$ & JMR & 50.76 & 13.22 & 2.34 & 8.69 & 7.92 & 9.98 & 2.37 & .39 & nd & 2.00 & .25 & .18 & nd & 98.10 \\
\hline BO-3 & ML & k3 & 3 & SW-24 & a & JMR & 51.11 & 13.53 & 2.20 & 8.66 & 7.34 & 10.21 & 2.54 & .44 & nd & 2.05 & .28 & .17 & nd & 98.53 \\
\hline $\mathrm{BO}-4$ & $\mathrm{ML}$ & k5-1907 & 3 & ML-121 & $a$ & JMR & 51.65 & 13.49 & 12.05 & nd & 7.47 & 10.49 & 2.17 & .36 & nd & 2.07 & .23 & .16 & nd & 100.14 \\
\hline BO-5 & ML & $k 3$ & 3 & SW-23 & $\mathrm{a}$ & JMR & 48.73 & 9.98 & 2.89 & 8.66 & 18.06 & 7.52 & 1.81 & .31 & nd & 1.46 & .20 & .16 & nd & 99.78 \\
\hline BO-7 & $\mathrm{ML}$ & k3 & 3 & SW-21 & a & JMR & 51.23 & 13.80 & 2.83 & 8.41 & 6.75 & 10.30 & 2.40 & .42 & nd & 2.20 & .26 & .17 & nd & 98.77 \\
\hline BP-1 & ML & k5-1868 & 3 & ML-86 & a & JMR & 50.74 & 12.82 & 12.13 & nd & 8.97 & 10.05 & 2.07 & .40 & nd & 2.04 & .23 & .17 & nd & 99.62 \\
\hline BP-2 & ML & k5-1868 & 3 & ML-87 & a & JMR & 47.38 & 9.40 & 12.55 & nd & 19.02 & 7.46 & 1.49 & .28 & nd & 1.49 & .18 & .15 & nd & 99.40 \\
\hline BP-3 & ML & k2 & 3 & SW-27 & a & JMR & 51.03 & 13.67 & 2.80 & 8.35 & 7.41 & 10.30 & 2.54 & .37 & nd & 1.93 & .25 & .18 & nd & 98.83 \\
\hline BP-4 & ML & k5-1868 & 3 & ML-120 & a & JMR & 48.32 & 10.10 & 12.28 & nd & 17.01 & 7.91 & 1.53 & .32 & nd & 1.58 & .19 & .16 & nd & 99.40 \\
\hline BP-5 & ML & $\mathrm{kc4}$ & 3 & SW-28 & a & JMR & 49.87 & 11.75 & 2.58 & 8.63 & 12.76 & 8.97 & 2.38 & .37 & nd & 1.85 & .23 & .17 & nd & 99.56 \\
\hline BP-6 & ML & k5-1887 & 3 & ML-113 & a & JMR & 51.59 & 13.34 & 12.06 & nd & 7.82 & 10.21 & 2.11 & .32 & nd & 1.96 & .21 & .16 & nd & 99.78 \\
\hline BP-13 & ML & k5-1907 & 3 & ML-118 & a & JMR & 51.25 & 13.51 & 12.03 & nd & 7.49 & 10.54 & 2.20 & .36 & nd & 2.06 & .22 & .16 & nd & 99.82 \\
\hline BQ-1 & ML & k1y & 3 & SW-31 & a & JMR & 50.57 & 13.37 & 2.73 & 8.05 & 7.23 & 10.09 & 1.72 & .41 & nd & 2.01 & .25 & .17 & nd & 96.60 \\
\hline $\mathrm{BQ}-2$ & ML. & $k$ & 3 & SW-17 & a & PWL & 50.34 & 12.87 & 2.38 & 8.64 & 8.10 & 10.05 & 2.56 & .34 & nd & 2.07 & .23 & .17 & nd & 97.75 \\
\hline$B Q-3$ & ML & $k$ & 3 & SW-16 & a & PWL & 49.87 & 13.31 & 1.50 & 9.44 & 7.11 & 10.95 & 2.42 & .34 & nd & 2.15 & .24 & .16 & nd & 97.49 \\
\hline$B Q-4$ & ML & k & 3 & SW-15 & a & PWL & 49.87 & 12.88 & 1.49 & 9.48 & 8.79 & 9.80 & 2.38 & .33 & nd & 1.98 & .22 & .17 & nd & 97.39 \\
\hline BQ-5 & ML & k & 3 & SW-18 & a & JMR & 49.97 & 13.99 & 2.56 & 9.22 & 5.68 & 9.90 & 2.97 & .51 & nd & 2.64 & .31 & .19 & nd & 97.94 \\
\hline$B Q-6$ & ML & K3 & 3 & SW-8 & a & JMR & 48.74 & 10.71 & 1.56 & 9.53 & 14.70 & 8.22 & 1.98 & .40 & nd & 1.75 & .25 & .16 & nd & 98.00 \\
\hline$B Q-7$ & ML & K3 & 3 & SW-7 & a & JMR & 50.61 & 13.04 & 1.53 & 9.08 & 7.78 & 9.99 & 2.50 & .50 & nd & 2.20 & .32 & .15 & nd & 97.70 \\
\hline $\mathrm{CN}-1$ & ML & k5-1919 & 3 & ML-HAW8 & a & JMR & 51.94 & 13.81 & 12.04 & nd & 7.14 & 10.64 & 2.31 & .38 & nd & 2.10 & .23 & .17 & nd & 100.76 \\
\hline $\mathrm{CO}-1$ & ML & k4 & 3 & SW-19 & a & JMR & 50.50 & 12.53 & 2.49 & 8.47 & 10.17 & 9.29 & 2.43 & .41 & nd & 1.87 & .27 & .17 & nd & 98.60 \\
\hline $\mathrm{CO}-2$ & ML & $\mathrm{k} 3$ & 3 & SW-3 & a & JMR & 48.69 & 10.21 & 2.27 & 9.21 & 17.08 & 7.75 & 1.75 & .30 & nd & 1.50 & .21 & nd & nd & 98.97 \\
\hline $\mathrm{CO}-4$ & $\mathrm{ML}$ & k5-1926 & 3 & ML-HAW9 & a & JMR & 51.69 & 13.48 & 11.47 & nd & 7.81 & 10.49 & 2.25 & .35 & nd & 2.04 & .24 & .17 & nd & 99.99 \\
\hline
\end{tabular}


Table 1.-Chemical analyses-Continued

\begin{tabular}{|c|c|c|c|c|c|c|c|c|c|c|c|c|c|c|c|c|c|c|c|c|}
\hline $\begin{array}{c}\text { Sample } \\
\text { locality } \\
\text { No. }\end{array}$ & $\begin{array}{l}\text { Vol- } \\
\text { cano }\end{array}$ & Unit & $\begin{array}{l}\text { Map } \\
\text { sheet } \\
\text { No. }\end{array}$ & $\begin{array}{l}\text { Laboratory } \\
\text { No. }\end{array}$ & Remark & Collector & $\mathrm{SiO}_{2}$ & $\mathrm{Al}_{2} \mathrm{O}_{3}$ & $\mathrm{Fe}_{2} \mathrm{O}_{3}$ & $\mathrm{FeO}$ & $\mathrm{MgO}$ & $\mathrm{CaO}$ & $\mathrm{Na}_{2} \mathrm{O}$ & $\mathrm{K}_{2} \mathrm{O}$ & $\mathrm{H}_{2} \mathrm{O}$ & $\mathrm{THO}_{2}$ & $\mathrm{P}_{2} \mathrm{O}_{5}$ & $\mathrm{MnO}$ & $\mathrm{CO}_{2}$ & Total \\
\hline $\mathrm{CP}-1$ & ML & k5-1887 & 3 & ML-97 & $\mathrm{a}$ & JMR & 51.31 & 13.07 & 12.01 & nd & 8.37 & 10.05 & 1.98 & .32 & nd & 1.92 & .20 & .17 & nd & 99.40 \\
\hline $\mathrm{CP}-2$ & ML & k5-1916 & 3 & ML-89 & a & JMR & 51.60 & 13.43 & 12.04 & nd & 7.62 & 10.45 & 2.23 & .36 & nd & 2.06 & .22 & .17 & nd & 100.18 \\
\hline $\mathrm{CP}-3$ & ML & k2 & 3 & D-305817 & b & MDJ & 51.70 & 13.90 & 12.40 & nd & 6.69 & 10.80 & 2.31 & .40 & .00 & 2.15 & .26 & .17 & nd & 100.78 \\
\hline CP-4 & ML & k5-1926 & 3 & ML-90 & $\mathrm{a}$ & JMR & 51.28 & 13.40 & 12.07 & nd & 7.79 & 10.49 & 2.15 & .35 & nd & 2.02 & .23 & .16 & nd & 99.94 \\
\hline CP-5 & ML & k1 & 3 & D-305818 & b & MDJ & 51.70 & 13.60 & 12.00 & nd & 7.84 & 10.40 & 2.32 & .42 & .00 & 2.05 & .26 & .17 & nd & 100.76 \\
\hline $\mathrm{CP}-6$ & ML & k2 & 3 & D-305819 & b & MDJ & 51.60 & 13.90 & 12.00 & nd & 7.34 & 10.80 & 2.29 & .39 & .00 & 2.08 & .25 & .17 & nd & 100.82 \\
\hline CP-7 & ML & k5-1887 & 3 & ML-95 & a & JMR & 51.93 & 13.29 & 12.16 & nd & 7.55 & 10.15 & 2.02 & .33 & nd & 1.99 & .21 & .17 & nd & 99.80 \\
\hline CP- 8 & ML & k2 & 3 & D-305820 & $\mathrm{b}$ & MDJ & 51.80 & 13.50 & 12.30 & nd & 7.47 & 10.70 & 2.21 & .40 & .04 & 2.08 & .25 & .17 & nd & 100.92 \\
\hline CP-9 & ML & $k 5-1887$ & 3 & ML-93 & a & JMR & 51.71 & 13.35 & 12.00 & nd & 7.50 & 10.07 & 2.13 & .33 & nd & 1.98 & .21 & .16 & nd & 99.44 \\
\hline CP-11 & $\mathrm{ML}$ & k4 & 3 & D-305821 & b & MDJ & 51.50 & 13.10 & 12.10 & nd & 9.05 & 9.92 & 2.34 & .50 & .00 & 2.29 & .31 & .16 & nd & 101.27 \\
\hline $\mathrm{CP}-12$ & ML & $k 3$ & 3 & D-305822 & b & MDJ & 51.80 & 13.90 & 12.20 & nd & 6.88 & 10.80 & 2.32 & .41 & .00 & 2.16 & .27 & .17 & nd & 100.91 \\
\hline CP- 13 & $\mathrm{ML}$ & k5-1907 & 3 & ML-91 & a & JMR & 51.76 & 13.51 & 11.97 & nd & 7.51 & 10.43 & 1.84 & .36 & nd & 2.04 & .22 & .17 & nd & 99.81 \\
\hline CP-14 & ML & k4 & 3 & SW-70 & a & JMR & 51.52 & 12.74 & 11.94 & nd & 9.33 & 9.64 & 2.35 & .41 & nd & 1.91 & .25 & .16 & nd & 100.25 \\
\hline CP-15 & ML & k5-1916 & 3 & ML-62A & a & JMR & 51.40 & 13.25 & 11.93 & nd & 7.63 & 10.42 & 2.09 & .36 & nd & 2.05 & .22 & .16 & nd & 99.51 \\
\hline CQ-1 & ML & k2 & 3 & SW-36 & a & JMR & 51.04 & 13.67 & 2.99 & 8.09 & 6.81 & 10.25 & 2.38 & .42 & nd & 2.09 & .26 & .18 & nd & 98.18 \\
\hline $\mathrm{CQ}-2$ & ML & $n$ & 3 & SW-99 & a & JMR & 51.18 & 14.19 & 12.67 & nd & 7.73 & 9.88 & 1.69 & .21 & nd & 2.31 & .21 & .18 & nd & 100.25 \\
\hline $\mathrm{CQ}-3$ & ML & $\mathrm{n}$ & 3 & SW-100 & a & JMR & 50.01 & 13.87 & 12.84 & nd & 8.63 & 10.16 & 1.75 & .06 & nd & 2.11 & .20 & .18 & nd & 99.81 \\
\hline$C Q-4$ & ML. & $\mathrm{n}$ & 3 & SW-101 & a & JMR & 51.34 & 13.79 & 12.37 & nd & 7.45 & 10.63 & 1.88 & .12 & nd & 2.05 & .19 & .18 & nd & 100.00 \\
\hline$C Q-5$ & ML & $\mathrm{n}$ & 3 & SW-98 & a & JMR & 50.76 & 13.72 & 12.93 & nd & 7.04 & 10.67 & 1.97 & .08 & nd & 2.25 & .22 & .18 & nd & 99.82 \\
\hline CQ-6 & $\overline{M L}$ & kiy & 3 & SW-9 & a & PWL & 51.55 & 13.70 & 2.99 & 8.13 & 7.19 & 10.23 & 2.94 & .45 & nd & 2.15 & .28 & .18 & nd & 99.79 \\
\hline $\mathrm{CQ}-8$ & ML & k1y & 3 & SW-10 & a & PWL & 50.70 & 13.83 & 1.88 & 8.99 & 6.45 & 10.26 & 2.84 & .45 & nd & 2.20 & .30 & .18 & nd & 98.08 \\
\hline$C Q-10$ & $\mathrm{KI}$ & p1o & 3 & D-287566 & b & CAN & 48.60 & 12.00 & 12.80 & nd & 12.90 & 9.60 & 1.97 & .32 & .00 & 2.29 & .22 & .17 & nd & 100.87 \\
\hline CQ-11 & ML & $n$ & 3 & SW-45 & a & PWL & 50.81 & 13.58 & 2.34 & 8.99 & 6.60 & 10.49 & 2.37 & .29 & nd & 2.29 & .22 & .17 & nd & 98.15 \\
\hline $\mathrm{CQ}-12$ & $\mathrm{ML}$ & $n$ & 3 & SW-46 & a & PWL & 51.08 & 13.91 & 12.75 & nd & 6.69 & 10.72 & 2.41 & .23 & nd & 2.34 & .24 & .18 & nd & 100.55 \\
\hline$C Q-13$ & ML & $n$ & 3 & SW-47 & a & PWL & 51.23 & 13.77 & 12.66 & nd & 6.56 & 10.62 & 2.06 & .25 & nd & 2.33 & .23 & .18 & nd & 99.89 \\
\hline CQ-14 & ML & $n$ & 3 & SW-48 & a & PWL & 50.39 & 14.27 & 12.04 & nd & 7.08 & 10.97 & 1.93 & .10 & nd & 2.29 & .19 & .18 & nd & 99.44 \\
\hline CQ-15 & ML & $n$ & 3 & SW-49 & a & PWL & 49.84 & 13.29 & 12.52 & nd & 9.19 & 10.23 & 1.76 & .07 & nd & 2.06 & .18 & .18 & nd & 99.32 \\
\hline$C Q-16$ & ML & $\mathrm{n}$ & 3 & SW-50 & a & PWL & 50.43 & 14.32 & 12.51 & nd & 7.49 & 10.57 & 1.71 & .10 & nd & 2.29 & .18 & .19 & nd & 99.79 \\
\hline$C Q-17$ & ML & $n$ & 3 & SW-53 & a & PWL & 49.99 & 14.46 & 12.99 & nd & 6.84 & 10.90 & 2.05 & .06 & nd & 2.36 & .22 & .19 & nd & 100.06 \\
\hline$C Q-18$ & ML & $n$ & 3 & SW-52 & a & PWL & 50.10 & 13.72 & 12.59 & nd & 9.38 & 9.85 & 2.13 & .10 & nd & 2.15 & .20 & .17 & nd & 100.39 \\
\hline CQ-19 & $\mathrm{ML}$ & k10 & 3 & SW-11 & a & JMR & 50.60 & 13.66 & 2.91 & 8.13 & 7.36 & 10.26 & 2.11 & .35 & nd & 2.02 & .24 & .17 & nd & 97.81 \\
\hline$C Q-20$ & $\overline{M L}$ & k1y & 3 & SW-89 & a & PWL & 51.77 & 13.60 & 12.48 & nd & 6.06 & 10.00 & 2.86 & .50 & nd & 2.47 & .34 & .19 & nd & 100.27 \\
\hline$C Q-21$ & $\mathrm{ML}$ & $\mathrm{k} 2$ & 3 & SW-12 & $a$ & PWL & 51.31 & 13.77 & 2.86 & 8.04 & 7.13 & 10.28 & 2.91 & .41 & nd & 2.06 & .25 & .17 & nd & 99.19 \\
\hline $\mathrm{CQ}-22$ & $\mathrm{ML}$ & k3 & 3 & SW-76 & a & PWL & 48.67 & 10.44 & 12.51 & nd & 15.42 & 7.89 & 2.01 & .42 & nd & 1.84 & .29 & .18 & nd & 99.67 \\
\hline $\mathrm{CQ}-23$ & $\mathrm{ML}$ & $n$ & 3 & SW-91 & a & JMR & 51.32 & 13.61 & 11.85 & nd & 7.43 & 10.77 & 1.88 & .23 & nd & 2.07 & .19 & .17 & nd & 99.52 \\
\hline $\mathrm{CQ}-24$ & $\mathrm{ML}$ & $n$ & 3 & SW-79 & a & PWL & 50.04 & 12.96 & 12.00 & nd & 10.03 & 10.62 & 2.03 & .21 & nd & 2.08 & .21 & .16 & nd & 100.34 \\
\hline $\mathrm{CQ}-25$ & ML. & $n$ & 3 & SW-80 & a & PWL & 51.39 & 13.90 & 12.02 & nd & 7.17 & 10.85 & 2.25 & .20 & nd & 2.13 & .21 & .18 & nd & 100.30 \\
\hline$C Q-26$ & ML & $n$ & 3 & SW-78 & a & PWL & 49.80 & 12.95 & 12.54 & nd & 10.26 & 10.02 & 1.91 & .15 & nd & 2.08 & .19 & .18 & nd & 100.08 \\
\hline $\mathrm{CQ}-27$ & ML & $n$ & 3 & SW-77 & a & PWL & 50.26 & 12.37 & 12.34 & nd & 11.23 & 9.59 & 1.92 & .25 & nd & 1.86 & .19 & .18 & nd & 100.19 \\
\hline$C Q-28$ & $\mathrm{ML}$ & $\mathrm{n}$ & 3 & SW-81 & a & PWL & 42.70 & 4.07 & 12.64 & nd & 34.90 & 3.26 & .37 & .08 & nd & .65 & .06 & .17 & nd & 98.90 \\
\hline
\end{tabular}


Table 1.-Chemical analyses-Continued

\begin{tabular}{|c|c|c|c|c|c|c|c|c|c|c|c|c|c|c|c|c|c|c|c|c|}
\hline $\begin{array}{c}\text { Sample } \\
\text { locality } \\
\text { No. }\end{array}$ & $\begin{array}{l}\text { Vol- } \\
\text { cano }\end{array}$ & Unit & $\begin{array}{c}\text { Map } \\
\text { sheet } \\
\text { No. }\end{array}$ & $\begin{array}{l}\text { Laboratory } \\
\text { No. }\end{array}$ & Remark & Collector & $\mathrm{SiO}_{2}$ & $\mathrm{Al}_{2} \mathrm{O}_{3}$ & $\mathrm{Fe}_{2} \mathrm{O}_{3}$ & $\mathrm{FeO}$ & $\mathrm{MgO}$ & $\mathrm{CaO}$ & $\mathrm{Na}_{2} \mathrm{O}$ & $\mathrm{K}_{2} \mathrm{O}$ & $\mathrm{H}_{2} \mathrm{O}$ & $\mathrm{TiO}_{2}$ & $\mathrm{P}_{2} \mathrm{O}_{5}$ & $\mathrm{MnO}$ & $\mathrm{CO}_{2}$ & Total \\
\hline CQ-29 & $\mathrm{ML}$ & $n$ & 3 & SW-90 & $a$ & JMR & 49.86 & 12.83 & 12.34 & nd & 9.80 & 10.52 & 1.64 & .21 & nd & 2.16 & .21 & .18 & nd & 99.75 \\
\hline$C Q-30$ & ML & $k 2$ & 3 & D-305823 & b & MDJ & 51.90 & 13.70 & 12.80 & nd & 6.69 & 10.70 & 2.34 & .40 & .00 & 2.22 & .27 & .18 & nd & 101.20 \\
\hline CR-2 & $\mathrm{KI}$ & pld3 & 3 & D-278805 & $b$ & CAN & 49.80 & 13.20 & 12.50 & nd & 8.39 & 11.10 & 2.23 & .48 & .00 & 2.62 & .26 & .17 & nd & 100.75 \\
\hline CR-3 & $\mathrm{KI}$ & p4o & 3 & D-315371 & b & CAN & 50.30 & 13.20 & 12.10 & nd & 8.04 & 11.70 & 2.21 & .44 & .00 & 2.57 & .25 & .16 & nd & 100.97 \\
\hline CR-4 & $\mathrm{KI}$ & p3 & 3 & D-278806 & $b$ & CAN & 50.50 & 12.50 & 12.60 & nd & 9.85 & 10.50 & 1.95 & .37 & .00 & 2.30 & .23 & .17 & nd & 100.97 \\
\hline CR-5 & ML & $\mathrm{K} 3$ & 3 & D-315391 & $\mathrm{b}$ & CAN & 52.10 & 13.60 & 12.00 & nd & 7.31 & 11.00 & 2.23 & .34 & .00 & 2.27 & .23 & .17 & nd & 101.25 \\
\hline CR-7 & $\mathrm{KI}$ & p4o & 3 & D-315390 & b & CAN & 50.00 & 13.00 & 12.50 & nd & 7.91 & 11.60 & 2.26 & .46 & .00 & 2.59 & .26 & .17 & nd & 100.75 \\
\hline CR-8 & KI & p4o & 3 & D-278707 & b & CAN & 51.50 & 13.70 & 12.20 & nd & 6.75 & 11.30 & 2.26 & .43 & .00 & 2.56 & .26 & .17 & nd & 101.13 \\
\hline CR-9 & KI & p4o & 3 & D-315389 & $\mathrm{b}$ & CAN & 50.50 & 12.70 & 12.70 & nd & 9.59 & 10.60 & 2.19 & .37 & .00 & 2.33 & .23 & .17 & nd & 101.38 \\
\hline CR-10 & ML & k1o & 3 & D-306264 & b & MDJ & 51.40 & 13.10 & 12.60 & nd & 8.57 & 10.40 & 2.15 & .33 & .00 & 2.03 & .22 & .17 & nd & 100.97 \\
\hline CR-11 & $\mathrm{KJ}$ & p3 & 3 & D-287563 & $b$ & CAN & 50.30 & 13.30 & 13.20 & nd & 7.81 & 11.10 & 2.23 & .44 & .00 & 2.50 & .25 & .18 & nd & 101.31 \\
\hline CR-13 & ML & $k$ & 3 & D-306263 & b & MDJ & 51.70 & 13.80 & 12.20 & nd & 6.99 & 10.60 & 2.33 & .41 & .00 & 2.10 & .26 & .17 & nd & 100.56 \\
\hline CR-14 & ML & $\mathrm{k} 3$ & 3 & D-306257 & b & MDJ & 52.00 & 13.50 & 12.10 & nd & 7.43 & 10.90 & 2.22 & .35 & .00 & 2.26 & .24 & .17 & nd & 101.17 \\
\hline CR-15 & KI & $p c 2$ & 3 & D-287565 & b & CAN & 51.20 & 13.40 & 14.10 & nd & 6.01 & 10.10 & 2.43 & .53 & .00 & 3.27 & .34 & .19 & nd & 101.57 \\
\hline CR-17 & ML & $k$ & 3 & D-306256 & b & MDJ & 50.90 & 13.70 & 12.30 & nd & 7.31 & 11.30 & 2.26 & .41 & .00 & 2.49 & .26 & .17 & nd & 101.10 \\
\hline CR-18 & $\mathrm{Kl}$ & p1y & 3 & D-287569 & b & CAN & 48.90 & 11.80 & 12.80 & nd & 14.20 & 9.32 & 1.74 & .32 & .00 & 2.08 & .20 & .18 & nd & 101.54 \\
\hline CR-19 & $\mathrm{KI}$ & $\mathrm{p3}$ & 3 & D-287564 & $b$ & CAN & 49.90 & 12.10 & 12.60 & nd & 11.60 & 9.90 & 1.97 & .33 & .00 & 2.12 & .20 & .17 & nd & 100.89 \\
\hline CR-20 & $\mathrm{KI}$ & pc3 & 3 & D-314264 & b & CAN & 49.10 & 11.60 & 12.70 & nd & 12.80 & 10.10 & 1.88 & .32 & .00 & 2.20 & .21 & .17 & nd & 101.08 \\
\hline CR-21 & $\mathrm{KI}$ & p5-1823 & 3 & D-287562 & $b$ & CAN & 47.30 & 10.10 & 13.10 & nd & 17.40 & 8.36 & 1.76 & .42 & .00 & 2.06 & .25 & .17 & nd & 100.92 \\
\hline CR-22 & $\mathrm{KI}$ & $\mathrm{p} 40$ & 3 & D-315397 & $b$ & CAN & 49.50 & 12.60 & 12.90 & nd & 9.39 & 11.10 & 2.14 & .44 & .00 & 2.50 & .25 & .17 & nd & 100.99 \\
\hline CR-23 & $\mathrm{KI}$ & p3 & 3 & D-315396 & b & CAN & 49.50 & 11.60 & 13.00 & nd & 12.40 & 9.71 & 2.00 & .37 & .00 & 2.27 & .24 & .17 & nd & 101.26 \\
\hline CR-24 & ML. & $k$ & 3 & D-306259 & $\mathrm{b}$ & MDJ & 51.50 & 13.60 & 12.30 & nd & 7.52 & 10.70 & 2.16 & .28 & .00 & 2.06 & .21 & .17 & nd & 100.50 \\
\hline CR-25 & $\mathrm{KI}$ & p2 & 3 & D-315398 & $b$ & CAN & 50.20 & 12.30 & 12.40 & nd & 11.00 & 10.20 & 2.02 & .32 & .00 & 1.95 & .21 & .17 & nd & 100.77 \\
\hline CR-26 & $\mathrm{KI}$ & p2 & 3 & D-287561 & b & CAN & 50.40 & 12.80 & 12.60 & nd & 8.95 & 10.50 & 2.14 & .45 & .15 & 2.61 & .27 & .17 & nd & 101.04 \\
\hline CR-27 & $\mathrm{KI}$ & p4o & 3 & D-315373 & b & CAN & 50.20 & 13.30 & 12.50 & nd & 7.75 & 11.80 & 2.25 & .45 & .00 & 2.62 & .26 & .17 & nd & 101.30 \\
\hline CR-28 & $\mathrm{KI}$ & $\mathrm{p} 4 \mathrm{y}$ & 3 & D-315372 & $b$ & CAN & 51.60 & 13.60 & 13.00 & nd & 6.58 & 10.20 & 2.50 & .54 & .00 & 2.94 & .31 & .17 & nd & 101.44 \\
\hline CR-29 & $\mathrm{KI}$ & p5-1823 & 3 & D-315393 & b & CAN & 50.30 & 13.30 & 13.20 & nd & 7.86 & 11.10 & 2.30 & .44 & .00 & 2.50 & .25 & .18 & nd & 101.43 \\
\hline CR-30 & $\mathrm{K}]$ & p3 & 3 & D-315392 & b & CAN & 50.10 & 12.60 & 12.80 & nd & 9.77 & 10.10 & 2.07 & .39 & .00 & 2.36 & .24 & .17 & nd & 100.60 \\
\hline CR-31 & KI & p4o & 3 & D-287084 & $b$ & MDJ & 50.70 & 13.70 & 12.40 & nd & 7.40 & 11.10 & 2.33 & .42 & .00 & 2.49 & .25 & .17 & nd & 100.96 \\
\hline CR-32 & $\mathrm{Kl}$ & p3 & 3 & D-315388 & $b$ & CAN & 49.80 & 12.50 & 12.80 & nd & 10.70 & 10.10 & 2.00 & .36 & .16 & 2.29 & .23 & .17 & nd & 101.11 \\
\hline CR-34 & ML & $\mathrm{k} 3$ & 3 & SW-13 & a & PWL & 51.36 & 13.71 & 2.28 & 8.72 & 7.03 & 10.29 & 2.43 & .39 & nd & 2.05 & .25 & .17 & nd & 98.68 \\
\hline CR-35 & ML & $\mathrm{K} 3$ & 3 & D-306258 & b & MDJ & 51.60 & 12.90 & 12.50 & nd & 8.54 & 10.60 & 2.17 & .39 & .00 & 2.37 & .26 & .17 & nd & 101.50 \\
\hline CR-36 & ML & $\mathrm{K} 3$ & 3 & D-287101 & b & MDJ & 50.80 & 13.00 & 12.90 & nd & 8.48 & 10.10 & 2.15 & .43 & .00 & 2.34 & .27 & .18 & nd & 100.65 \\
\hline CR-37 & KI & p3 & 3 & D-278799 & b & CAN & 51.60 & 13.30 & 13.00 & nd & 6.11 & 9.46 & 2.57 & .74 & .00 & 3.01 & .40 & .18 & nd & 100.37 \\
\hline CR-39 & $\mathrm{KI}$ & p4o & 3 & D-278800 & b & CAN & 50.40 & 13.20 & 12.50 & nd & 7.46 & 11.60 & 2.10 & .46 & .00 & 2.62 & .27 & .17 & nd & 100.78 \\
\hline CR-40 & $\mathrm{KI}$ & $\mathrm{p} 3$ & 3 & D-278801 & $b$ & CAN & 52.10 & 13.10 & 13.80 & nd & 5.24 & 9.01 & 2.77 & .87 & .00 & 3.58 & .45 & .18 & nd & 101.10 \\
\hline CR-41 & KI & p4y & 3 & D-278802 & b & CAN & 50.80 & 12.80 & 12.60 & nd & 8.77 & 10.60 & 2.09 & .38 & .00 & 2.38 & .24 & .17 & nd & 100.83 \\
\hline CR-42 & $\mathrm{KI}$ & p4o & 3 & D-278803 & $b$ & CAN & 50.70 & 13.10 & 12.40 & nd & 7.87 & 11.50 & 2.09 & .43 & .00 & 2.51 & .25 & .17 & nd & 101.02 \\
\hline CR-43 & $\mathrm{KI}$ & p4y & 3 & D-278804 & b & CAN & 51.30 & 13.50 & 12.40 & nd & 6.72 & 10.80 & 2.28 & .48 & .00 & 2.73 & .29 & .17 & nd & 100.67 \\
\hline CR-44 & ML & $\mathrm{k} 3$ & 3 & D-287082 & b & MDJ & 51.10 & 12.80 & 12.50 & nd & 8.72 & 10.20 & 2.12 & .41 & .00 & 2.39 & .26 & .17 & nd & 100.67 \\
\hline
\end{tabular}


Table 1.-Chemical analyses-Continued

\begin{tabular}{|c|c|c|c|c|c|c|c|c|c|c|c|c|c|c|c|c|c|c|c|c|}
\hline $\begin{array}{l}\text { Sample } \\
\text { locality } \\
\text { No. }\end{array}$ & $\begin{array}{l}\text { Vol- } \\
\text { cano }\end{array}$ & Unit & $\begin{array}{c}\text { Map } \\
\text { sheet } \\
\text { No. }\end{array}$ & $\begin{array}{c}\text { Laboratory } \\
\text { No. }\end{array}$ & Remark & Collector & $\mathrm{SiO}_{2}$ & $\mathrm{Al}_{2} \mathrm{O}_{3}$ & $\mathrm{Fe}_{2} \mathrm{O}_{3}$ & $\mathrm{FeO}$ & $\mathrm{MgO}$ & $\mathrm{CaO}$ & $\mathrm{Na}_{2} \mathrm{O}$ & $\mathrm{K}_{2} \mathrm{O}$ & $\mathrm{H}_{2} \mathrm{O}$ & $\mathrm{TiO}_{2}$ & $\mathrm{P}_{2} \mathrm{O}_{5}$ & $\mathrm{MnO}$ & $\mathrm{CO}_{2}$ & Total \\
\hline CR-45 & $\mathrm{ML}$ & $k$ & 3 & D-306261 & $\mathrm{b}$ & MDJ & 51.20 & 13.20 & 12.40 & nd & 8.41 & 10.50 & 2.04 & .26 & .19 & 2.00 & .20 & .17 & nd & 100.57 \\
\hline CR-46 & $\mathrm{KI}$ & p3 & 3 & D-287554 & b & $\mathrm{CAN}$ & 49.80 & 12.00 & 12.40 & nd & 12.80 & 9.63 & 1.93 & .30 & .00 & 2.13 & .20 & .17 & nd & 101.36 \\
\hline CS-1 & $\mathrm{KI}$ & p3 & 2 & D-284994 & b & CAN & 50.60 & 12.60 & 12.90 & nd & 9.29 & 10.70 & 2.10 & .36 & .00 & 2.39 & .24 & .18 & nd & 101.36 \\
\hline CS-2 & $\mathrm{KI}$ & p3 & 2 & D-284995 & b & CAN & 49.90 & 12.30 & 12.80 & nd & 10.60 & 10.40 & 1.96 & .34 & .01 & 2.29 & .22 & .18 & nd & 101.00 \\
\hline CS-3 & $\mathrm{KI}$ & p4o & 2 & D-284996 & $b$ & CAN & 50.60 & 13.30 & 12.60 & nd & 7.45 & 11.50 & 2.23 & .45 & .00 & 2.61 & .26 & .17 & nd & 101.17 \\
\hline $\mathrm{CS}-4$ & $\mathrm{Kl}$ & p4y & 2 & D-284999 & b & CAN & 51.60 & 13.70 & 12.40 & nd & 6.82 & 10.90 & 2.43 & .50 & .00 & 2.73 & .29 & .17 & nd & 101.54 \\
\hline CS-7 & $\mathrm{KI}$ & p4o & 2 & D-315409 & b & CAN & 50.80 & 13.30 & 12.50 & nd & 7.14 & 11.60 & 2.30 & .45 & .00 & 2.59 & .25 & .17 & nd & 101.10 \\
\hline CS-8 & $\mathrm{Kl}$ & p3 & 2 & D-314263 & b & CAN & 50.90 & 13.30 & 13.80 & nd & 5.73 & 9.86 & 2.55 & .64 & .00 & 3.38 & .39 & .18 & nd & 100.73 \\
\hline CS-9 & $\mathrm{KI}$ & hi & 2 & D-314260 & b & CAN & 51.30 & 13.70 & 12.30 & nd & 7.06 & 10.90 & 2.23 & .46 & .00 & 2.70 & .27 & .17 & nd & 101.09 \\
\hline CS-10 & $\mathrm{Kl}$ & hi & 2 & D-314259 & b & CAN & 50.60 & 13.50 & 12.70 & nd & 6.97 & 11.20 & 2.15 & .44 & .00 & 2.75 & .26 & .17 & nd & 100.74 \\
\hline CS-11 & $\mathrm{K}]$ & p2 & 2 & D-314265 & b & CAN & 51.20 & 13.80 & 12.40 & nd & 6.92 & 10.90 & 2.29 & .47 & .00 & 2.71 & .28 & .17 & nd & 101.14 \\
\hline DN-1 & $\mathrm{ML}$ & k5-1950 & 3 & ML-55 & a & JMR & 50.91 & 13.11 & 12.20 & nd & 8.65 & 10.17 & 1.83 & .35 & nd & 1.94 & .21 & .16 & nd & 99.53 \\
\hline $\mathrm{DN}-2$ & ML & k5-1950 & 3 & ML-122 & a & JMR & 50.68 & 13.03 & 12.20 & nd & 8.62 & 10.13 & 2.09 & .35 & nd & 1.93 & .22 & .18 & nd & 99.43 \\
\hline DN-3 & ML & k5-1950 & 3 & ML-56 & a & JMR & 51.21 & 13.14 & 12.06 & nd & 8.38 & 10.21 & 2.17 & .37 & nd & 1.97 & .22 & .17 & nd & 99.90 \\
\hline DO-1 & ML & k5 & 3 & ML-102 & a & PWL & 51.49 & 13.16 & 12.11 & nd & 8.26 & 10.00 & 2.01 & .34 & nd & 1.98 & .22 & .17 & nd & 99.74 \\
\hline DP-1 & $\mathrm{ML}$ & k4 & 3 & ML-107 & a & PWL & 51.68 & 13.45 & 12.11 & nd & 7.44 & 10.46 & 2.09 & .35 & nd & 2.06 & .22 & .16 & nd & 100.02 \\
\hline DP-2 & ML & k5-1926 & 3 & ML-64C & a & JMR & 51.41 & 13.31 & 12.04 & nd & 7.55 & 10.40 & 2.23 & .36 & nd & 2.03 & .22 & .16 & nd & 99.71 \\
\hline DP-3 & ML & k5-1950 & 3 & ML-66 & $\mathrm{a}$ & JMR & 50.31 & 11.99 & 11.90 & nd & 12.10 & 9.34 & 2.15 & .31 & nd & 1.71 & .18 & .17 & nd & 100.16 \\
\hline DP-4 & $\mathrm{ML}$ & kc5-1919 & 3 & ML-63 & a & JMR & 51.64 & 13.61 & 12.07 & nd & 7.32 & 10.48 & 2.20 & .37 & nd & 2.07 & .23 & .18 & nd & 100.17 \\
\hline DP-5 & $\mathrm{ML}$ & k2 & 3 & SW-60 & a & JMR & 51.13 & 13.20 & 11.83 & nd & 8.16 & 10.03 & 2.43 & .44 & nd & 2.03 & .28 & .17 & nd & 99.70 \\
\hline DP-7 & ML & k5-1950 & 3 & ML-67 & a & JMR & 50.74 & 12.87 & 12.10 & nd & 9.41 & 9.98 & 2.01 & .34 & nd & 1.89 & .21 & .16 & nd & 99.71 \\
\hline DP-8 & ML & $k 5$ & 3 & ML-103 & a & PWL & 51.62 & 13.14 & 12.10 & nd & 8.27 & 10.00 & 2.03 & .34 & nd & 1.98 & .22 & .16 & nd & 99.86 \\
\hline DP-9 & $\mathrm{ML}$ & k5-1950 & 3 & ML-105 & a & PWL & 51.10 & 13.69 & 12.18 & nd & 6.65 & 10.60 & 2.45 & .39 & nd & 2.08 & .23 & .17 & nd & 99.54 \\
\hline DP-10 & ML & kc3 & 3 & SW-85 & a & PWL & 48.78 & 10.49 & 12.05 & nd & 15.54 & 8.06 & 2.04 & .38 & nd & 1.71 & .27 & .18 & nd & 99.50 \\
\hline DP-11 & $\mathrm{ML}$ & $k 2$ & 3 & ML-104 & $a$ & PWL & 51.75 & 13.75 & 12.12 & nd & 6.88 & 10.52 & 2.35 & .38 & nd & 2.11 & .23 & .17 & nd & 100.26 \\
\hline DP-12 & ML & K3 & 3 & SW-83 & a & PWL & 51.81 & 13.83 & 12.01 & nd & 6.47 & 10.40 & 2.51 & .43 & nd & 2.14 & .28 & .16 & nd & 100.04 \\
\hline DQ-1 & ML & k2 & 3 & D-305813 & b & MDJ & 51.50 & 13.90 & 12.20 & nd & 6.85 & 10.70 & 2.35 & .42 & .06 & 2.18 & .27 & .17 & nd & 100.60 \\
\hline $\mathrm{DQ}-2$ & $\mathrm{ML}$ & $\overline{K B}$ & 3 & D-305812 & $\mathrm{b}$ & MDJ & 51.60 & 14.20 & 12.70 & nd & 6.31 & 10.80 & 2.39 & .41 & .00 & 2.25 & .27 & .17 & nd & 101.10 \\
\hline$D Q-3$ & ML & $k 2$ & 3 & D-305811 & b & MDJ & 51.90 & 13.90 & 12.20 & nd & 7.23 & 10.80 & 2.27 & .37 & .00 & 2.05 & .24 & .17 & nd & 101.13 \\
\hline$D Q-5$ & $\mathrm{ML}$ & k2 & 3 & D-305815 & b & MDJ & 51.80 & 14.00 & 12.20 & nd & 7.02 & 10.80 & 2.32 & .37 & .00 & 2.06 & .25 & .17 & nd & 100.99 \\
\hline DQ-6 & ML & k2 & 3 & D-305824 & $b$ & MDJ & 51.50 & 14.10 & 11.90 & nd & 7.18 & 11.10 & 2.31 & .39 & .03 & 2.02 & .25 & .17 & nd & 100.95 \\
\hline $\mathrm{DQ}-7$ & ML & k5-1950 & 3 & ML-68A & a & JMR & 51.61 & 13.88 & 12.06 & nd & 6.66 & 10.57 & 2.19 & .39 & nd & 2.11 & .24 & .17 & nd & 99.88 \\
\hline DQ-8 & ML & k3 & 3 & D-305810 & b & MDJ & 52.10 & 13.70 & 11.90 & nd & 6.88 & 11.00 & 2.20 & .40 & .00 & 2.42 & .26 & .17 & nd & 101.03 \\
\hline DQ-9 & ML & k4 & 3 & SW-62 & a & JMR & 51.38 & 13.79 & 12.11 & nd & 6.14 & 10.17 & 2.59 & .52 & nd & 2.45 & .34 & .18 & nd & 99.67 \\
\hline DQ-10 & $\mathrm{ML}$ & $k 2$ & 3 & D-305809 & b & MDJ & 50.10 & 11.90 & 11.80 & nd & 12.80 & 9.27 & 1.94 & .30 & .44 & 1.91 & .20 & .16 & nd & 100.82 \\
\hline$D Q-11$ & ML & K3 & 3 & D-305816 & $b$ & MDJ & 52.00 & 13.60 & 11.90 & nd & 7.20 & 10.90 & 2.25 & .35 & .00 & 2.27 & .24 & .17 & nd & 100.88 \\
\hline DR-2 & $\mathrm{KI}$ & p4y & 3 & D-314262 & $\mathrm{b}$ & $\mathrm{CAN}$ & 53.30 & 13.10 & 12.30 & nd & 5.92 & 8.41 & 2.84 & 1.00 & .06 & 2.68 & .57 & .17 & nd & 100.35 \\
\hline DR-3 & $\mathrm{ML}$ & $\mathrm{K} 3$ & 3 & D-306262 & $b$ & MDJ & 50.60 & 13.10 & 12.80 & nd & 8.51 & 10.20 & 2.24 & .43 & .00 & 2.31 & .29 & .17 & nd & 100.65 \\
\hline DR-4 & $\mathrm{K}]$ & p4y & 3 & D-314257 & b & CAN & 51.40 & 13.80 & 12.70 & nd & 6.23 & 10.40 & 2.46 & .59 & .00 & 3.21 & .34 & .17 & nd & 101.30 \\
\hline DR-6 & $\mathrm{KI}$ & p4o & 3 & D-315402 & b & CAN & 50.20 & 13.10 & 12.80 & nd & 7.70 & 11.60 & 2.23 & .46 & .00 & 2.65 & .26 & .17 & nd & 101.17 \\
\hline
\end{tabular}


Table 1.-Chemical analyses-Continued

\begin{tabular}{|c|c|c|c|c|c|c|c|c|c|c|c|c|c|c|c|c|c|c|c|c|}
\hline $\begin{array}{l}\text { Sample } \\
\text { locality } \\
\text { No. }\end{array}$ & $\begin{array}{l}\text { Vol- } \\
\text { cano }\end{array}$ & Unit & $\begin{array}{c}\text { Map } \\
\text { sheet } \\
\text { No. }\end{array}$ & $\begin{array}{c}\text { Laboratory } \\
\text { No. }\end{array}$ & Remark & Collector & $\mathrm{SiO}_{2}$ & $\mathrm{Al}_{2} \mathrm{O}_{3}$ & $\mathrm{Fe}_{2} \mathrm{O}_{3}$ & $\mathrm{FeO}$ & $\mathrm{MgO}$ & $\mathrm{CaO}$ & $\mathrm{Na}_{2} \mathrm{O}$ & $\mathrm{K}_{2} \mathrm{O}$ & $\mathrm{H}_{2} \mathrm{O}$ & $\mathrm{TiO}_{2}$ & $\mathrm{P}_{2} \mathrm{O}_{5}$ & $\mathrm{MnO}$ & $\mathrm{CO}_{2}$ & Total \\
\hline DR-7 & $\mathrm{KI}$ & $\mathrm{p} 4 \mathrm{y}$ & 3 & D-315404 & $\mathrm{b}$ & $\overline{\mathrm{CAN}}$ & 52.80 & 13.00 & 13.10 & nd & 4.85 & 8.18 & 3.03 & 1.04 & .41 & 3.17 & .45 & .17 & nd & 100.20 \\
\hline DR-8 & KI & p4y & 3 & D-287549 & b & CAN & 51.40 & 13.90 & 12.60 & nd & 6.12 & 10.40 & 2.52 & .58 & .00 & 2.92 & .33 & .17 & nd & 100.94 \\
\hline DR-9 & $\mathrm{K} l$ & p4y & 3 & D-287550 & b & CAN & 51.70 & 13.50 & 14.20 & nd & 5.26 & 9.50 & 2.71 & .71 & .00 & 3.59 & .42 & .19 & nd & 101.78 \\
\hline DR-10 & $\mathrm{KI}$ & p3 & 3 & D-276886 & b & CAN & 49.90 & 12.50 & 12.50 & nd & 10.30 & 10.30 & 2.09 & .33 & .08 & 2.17 & .22 & .17 & nd & 100.56 \\
\hline LR-11 & ML & $\mathrm{K} 3$ & 3 & D-287095 & b & MDJ & 51.80 & 13.50 & 12.10 & nd & 7.57 & 10.80 & 2.07 & .34 & .00 & 2.28 & .23 & .17 & nd & 100.86 \\
\hline DR-12 & $\mathrm{KI}$ & p4y & 3 & D-276885 & b & CAN & 50.40 & 12.80 & 12.80 & nd & 9.03 & 10.60 & 2.22 & .37 & .00 & 2.41 & .24 & .17 & nd & 101.04 \\
\hline DR-13 & $\mathrm{KI}$ & p4y & 3 & D-287551 & b & CAN & 53.50 & 13.40 & 13.80 & nd & 3.95 & 7.96 & 3.16 & 1.12 & .00 & 3.52 & .58 & .18 & nd & 101.17 \\
\hline DR-14 & $\mathrm{ML}$ & k3 & 3 & D-306260 & b & MDJ & 51.80 & 13.40 & 12.10 & nd & 7.32 & 10.80 & 2.29 & .42 & .00 & 2.44 & .27 & .17 & nd & 101.01 \\
\hline CR-15 & $\mathrm{KI}$ & p4o & 3 & D-276887 & b & CAN & 51.20 & 14.30 & 11.70 & nd & 7.00 & 11.50 & 2.40 & .38 & .00 & 2.37 & .24 & .16 & nd & 101.25 \\
\hline DR-16 & ML & $\mathrm{kB}$ & 3 & D-287090 & $b$ & MDJ & 50.10 & 11.40 & 13.10 & nd & 13.00 & 9.37 & 1.82 & .30 & .00 & 1.95 & .20 & .18 & nd & 101.42 \\
\hline DR-17 & KI & $\mathrm{p} 4 \mathrm{y}$ & 3 & D-315405 & $b$ & CAN & 55.70 & 13.20 & 11.00 & nd & 6.05 & 7.53 & 3.13 & 1.25 & .00 & 2.49 & .34 & .15 & nd & 100.84 \\
\hline DR-19 & KI & pc4y & 3 & D-315406 & b & CAN & 51.20 & 13.50 & 12.40 & nd & 7.37 & 11.10 & 2.34 & .41 & .00 & 2.47 & .24 & .17 & nd & 101.20 \\
\hline DR-20 & $\mathrm{KI}$ & p3 & 3 & D-315407 & b & CAN & 50.50 & 12.90 & 12.50 & nd & 8.78 & 11.00 & 2.22 & .41 & .00 & 2.40 & .23 & .17 & nd & 101.11 \\
\hline DR-21 & ML & $\mathrm{k} 3$ & 3 & D-287094 & b & MDJ & 51.80 & 13.40 & 11.80 & nd & 7.84 & 10.90 & 2.10 & .35 & .00 & 2.35 & .23 & .17 & nd & 100.94 \\
\hline DR-22 & ML & $\mathrm{k} 3$ & 3 & D-276888 & b & CAN & 51.90 & 13.40 & 11.70 & nd & 7.68 & 10.90 & 2.16 & .35 & .00 & 2.34 & .25 & .16 & nd & 100.84 \\
\hline DR-23 & $\mathrm{KI}$ & p3 & 3 & D-276882 & b & CAN & 50.30 & 12.60 & 12.60 & nd & 10.30 & 10.30 & 2.08 & .34 & .00 & 2.22 & .22 & .17 & nd & 101.13 \\
\hline DR-25 & $\mathrm{KI}$ & p4y & 3 & D-276883 & b & CAN & 51.10 & 13.60 & 12.20 & nd & 7.39 & 11.50 & 2.27 & .41 & .00 & 2.61 & .25 & .17 & nd & 101.50 \\
\hline DR-27 & $\mathrm{KI}$ & p3 & 3 & D-315408 & b & CAN & 50.70 & 13.30 & 12.50 & nd & 7.72 & 11.20 & 2.24 & .44 & .00 & 2.51 & .24 & .17 & nd & 101.02 \\
\hline DR-28 & KI & p4y & 3 & D-276881 & b & CAN & 50.90 & 13.50 & 12.30 & nd & 7.24 & 11.40 & 2.26 & .40 & .00 & 2.62 & .26 & .17 & nd & 101.05 \\
\hline DR-29 & $\mathrm{KI}$ & p3 & 3 & D-276895 & $\mathrm{b}$ & CAN & 48.60 & 10.60 & 12.50 & nd & 15.30 & 9.38 & 1.80 & .31 & .00 & 2.04 & .20 & .17 & nd & 100.90 \\
\hline DR-30 & $\mathrm{Kl}$ & p5-1919 & 3 & D-276897 & $b$ & CAN & 49.80 & 13.00 & 12.70 & nd & 8.11 & 11.50 & 2.24 & .50 & .00 & 2.85 & .28 & .18 & nd & 101.16 \\
\hline DR-31 & $\mathrm{KI}$ & p4o & 3 & D-287594 & b & CAN & 51.10 & 13.40 & 12.50 & nd & 7.44 & 11.20 & 2.16 & .41 & .00 & 2.50 & .26 & .18 & nd & 101.15 \\
\hline DR-32 & KI & p4o & 3 & D-276884 & b & CAN & 50.80 & 13.60 & 12.40 & nd & 7.28 & 11.10 & 2.34 & .41 & .00 & 2.52 & .26 & .17 & nd & 100.88 \\
\hline DR-33 & $\mathrm{KI}$ & p5-1919 & 3 & D-276902 & b & CAN & 49.90 & 13.20 & 12.40 & nd & 7.96 & 11.60 & 2.23 & .49 & .00 & 2.77 & .28 & .17 & nd & 101.00 \\
\hline DR-36 & $\mathrm{KI}$ & p5-1868 & 3 & D-276876 & b & CAN & 50.80 & 13.20 & 12.20 & nd & 8.42 & 11.00 & 2.24 & .43 & .00 & 2.60 & .26 & .17 & nd & 101.32 \\
\hline DR-38 & $\mathrm{K}$ & p4y & 3 & D-276898 & $\mathrm{b}$ & CAN & 50.80 & 13.50 & 12.10 & nd & 7.09 & 11.30 & 2.25 & .40 & .00 & 2.63 & .25 & .17 & nd & 100.49 \\
\hline DR-39 & $\mathrm{KI}$ & p4o & 3 & D-276905 & $\mathrm{b}$ & CAN & 50.70 & 13.20 & 12.60 & nd & 7.88 & 11.30 & 2.24 & .43 & .00 & 2.52 & .25 & .17 & nd & 101.29 \\
\hline DR-40 & KI & p5-1919 & 3 & D-276903 & b & CAN & 49.90 & 13.20 & 12.50 & nd & 8.17 & 11.50 & 2.11 & .49 & .00 & 2.79 & .28 & .17 & nd & 101.11 \\
\hline DR-41 & $\mathrm{KI}$ & p4o & 3 & D-276904 & b & CAN & 50.70 & 13.40 & 12.60 & nd & 7.16 & 11.20 & 2.29 & .45 & .05 & 2.61 & .26 & .17 & nd & 100.89 \\
\hline DR-42 & ML & $\mathrm{k} 3$ & 3 & D-287091 & $b$ & MDJ & 51.80 & 13.70 & 12.10 & nd & 6.98 & 11.10 & 2.16 & .40 & .00 & 2.30 & .24 & .17 & nd & 100.95 \\
\hline DR-44 & ML & $\mathrm{k} 3$ & 3 & D-287086 & b & MDJ & 51.50 & 13.60 & 11.90 & nd & 8.03 & 10.80 & 2.21 & .41 & .00 & 2.26 & .26 & .17 & nd & 101.14 \\
\hline DR-45 & ML & $k 2$ & 3 & D-287097 & b & MDJ & 49.90 & 11.10 & 12.20 & nd & 14.80 & 8.70 & 1.81 & .29 & .00 & 1.82 & .19 & .17 & nd & 100.98 \\
\hline DR-46 & $\mathrm{KI}$ & $\mathrm{p} 4 \mathrm{o}$ & 3 & D-276879 & $b$ & CAN & 51.40 & 13.70 & 12.10 & nd & 7.11 & 11.20 & 2.30 & .40 & .00 & 2.39 & .25 & .17 & nd & 101.02 \\
\hline DR-47 & ML & $\mathrm{k} 3$ & 3 & D-287103 & b & MDJ & 51.60 & 13.10 & 12.20 & nd & 8.64 & 10.60 & 2.01 & .34 & .00 & 2.26 & .23 & .17 & nd & 101.15 \\
\hline DR-48 & $\mathrm{KI}$ & p4o & 3 & D-276877 & b & CAN & 51.50 & 14.10 & 12.20 & nd & 6.56 & 11.10 & 2.47 & .43 & .00 & 2.52 & .27 & .17 & nd & 101.32 \\
\hline DR-49 & ML & $\mathrm{k} 3$ & 3 & D-287102 & b & MDJ & 48.40 & 8.77 & 13.10 & nd & 19.70 & 7.31 & 1.39 & .22 & .00 & 1.51 & .15 & .18 & nd & 100.73 \\
\hline DR-50 & ML & $\mathrm{k} 3$ & 3 & D-287081 & b & MDJ & 51.60 & 13.50 & 12.20 & nd & 7.91 & 10.70 & 2.18 & .38 & .00 & 2.33 & .23 & .17 & nd & 101.20 \\
\hline DR-51 & ML & $\mathrm{k} 3$ & 3 & D-287092 & $\mathrm{b}$ & MDJ & 50.00 & 10.40 & 13.50 & nd & 13.80 & 8.67 & 1.86 & .41 & .00 & 2.41 & .27 & .18 & nd & 101.50 \\
\hline DR-52 & ML & k10 & 3 & D-287096 & b & MDJ & 51.00 & 13.60 & 12.50 & nd & 7.60 & 11.30 & 2.13 & .33 & .00 & 2.20 & .22 & .18 & nd & 101.06 \\
\hline DR-53 & ML & k10 & 3 & D-287099 & b & MDJ & 51.60 & 13.90 & 12.50 & nd & 6.51 & 10.50 & 2.26 & .39 & .00 & 2.45 & .26 & .17 & nd & 100.54 \\
\hline
\end{tabular}


Table 1.-Chemical analyses-Continued

\begin{tabular}{|c|c|c|c|c|c|c|c|c|c|c|c|c|c|c|c|c|c|c|c|c|}
\hline $\begin{array}{l}\text { Sample } \\
\text { locality } \\
\text { No. }\end{array}$ & $\begin{array}{l}\text { Vol- } \\
\text { cano }\end{array}$ & Unit & $\begin{array}{c}\text { Map } \\
\text { sheet } \\
\text { No. }\end{array}$ & $\begin{array}{l}\text { Laboratory } \\
\text { No. }\end{array}$ & Remark & Collector & $\mathrm{SiO}_{2}$ & $\mathrm{Al}_{2} \mathrm{O}_{3}$ & $\mathrm{Fe}_{2} \mathrm{O}_{3}$ & $\mathrm{FeO}$ & $\mathrm{MgO}$ & $\mathrm{CaO}$ & $\mathrm{Na}_{2} \mathrm{O}$ & $\mathrm{K}_{2} \mathrm{O}$ & $\mathrm{H}_{2} \mathrm{O}$ & $\mathrm{TiO}_{2}$ & $\mathrm{P}_{2} \mathrm{O}_{5}$ & $\mathrm{MnO}$ & $\mathrm{CO}_{2}$ & Total \\
\hline DR-54 & ML & $\mathrm{k} 3$ & 3 & D-287093 & $\mathrm{b}$ & MDJ & 51.40 & 13.00 & 12.10 & nd & 8.78 & 10.50 & 2.06 & .34 & .00 & 2.27 & .23 & .17 & nd & 100.85 \\
\hline DR-55 & ML & $x 3$ & 3 & D-287080 & b & MDJ & 51.80 & 13.70 & 11.90 & nd & 6.87 & 10.70 & 2.27 & .44 & .00 & 2.45 & .27 & .16 & nd & 100.56 \\
\hline DR-56 & ML & $\mathrm{k} 3$ & 3 & D-276878 & b & CAN & 51.40 & 14.40 & 12.00 & nd & 6.37 & 10.80 & 2.44 & .38 & .00 & 2.34 & .25 & .16 & nd & 100.54 \\
\hline DS- 1 & $\mathrm{Kl}$ & $\mathrm{p} 4 \mathrm{y}$ & 2 & D-287598 & b & CAN & 51.80 & 13.10 & 14.40 & nd & 4.81 & 8.89 & 2.83 & .86 & .00 & 3.84 & .51 & .19 & nd & 101.23 \\
\hline DS-2 & $\mathrm{Kl}$ & p4o & 2 & D-287573 & b & CAN & 51.20 & 13.90 & 12.10 & nd & 7.14 & 11.30 & 2.28 & .40 & .00 & 2.40 & .25 & .17 & nd & 101.14 \\
\hline DS-3 & KI & p3 & 2 & D-287578 & b & CAN & 49.80 & 11.90 & 12.80 & nd & 11.90 & 10.20 & 1.88 & .33 & .00 & 2.24 & .21 & .17 & nd & 101.43 \\
\hline DS-4 & $\mathrm{Kl}$ & p3 & 2 & D-287577 & b & CAN & 50.50 & 13.20 & 12.60 & nd & 8.00 & 11.10 & 2.17 & .40 & .00 & 2.53 & .25 & .18 & nd & 100.93 \\
\hline DS-5 & $\mathrm{KI}$ & p4o & 2 & D-287574 & b & CAN & 51.30 & 13.90 & 12.20 & nd & 6.88 & 11.10 & 2.32 & .45 & .06 & 2.51 & .27 & .17 & nd & 101.16 \\
\hline DS-6 & $\mathrm{KI}$ & p2 & 2 & D-287579 & b & CAN & 50.90 & 12.90 & 12.80 & nd & 8.33 & 11.00 & 2.13 & .41 & .00 & 2.49 & .24 & .18 & nd & 101.38 \\
\hline DS-7 & $\mathrm{KI}$ & $\mathrm{p} 40$ & 2 & D-287553 & b & CAN & 49.00 & 11.30 & 13.30 & nd & 12.60 & 9.48 & 1.94 & .44 & .00 & 2.46 & .26 & .18 & nd & 100.96 \\
\hline DS-8 & $\mathrm{KI}$ & $\mathrm{p} 4 \mathrm{y}$ & 2 & D-287552 & b & CAN & 52.00 & 13.30 & 14.10 & nd & 4.84 & 8.98 & 2.90 & .87 & .00 & 3.84 & .50 & .19 & nd & 101.52 \\
\hline DS-9 & $\mathrm{Kl}$ & p3 & 2 & D-287559 & b & CAN & 50.90 & 13.40 & 12.40 & nd & 7.90 & 11.40 & 2.14 & .39 & .00 & 2.41 & .23 & .17 & nd & 101.34 \\
\hline DS- 10 & $\mathrm{KI}$ & p4o & 2 & D-287587 & b & CAN & 50.50 & 13.40 & 12.70 & nd & 7.50 & 11.70 & 2.15 & .46 & .00 & 2.66 & .26 & .18 & nd & 101.51 \\
\hline DS- 11 & $\mathrm{KI}$ & p4y & 2 & D-287588 & b & CAN & 52.10 & 13.20 & 14.20 & nd & 4.81 & 8.87 & 2.93 & .88 & .00 & 3.94 & .51 & .19 & nd & 101.63 \\
\hline DS-12 & $\mathrm{KI}$ & p3 & 2 & D-285000 & b & CAN & 48.90 & 11.70 & 13.30 & nd & 12.60 & 10.00 & 1.90 & .29 & .00 & 2.19 & .21 & .18 & nd & 101.27 \\
\hline DS-13 & $\mathrm{KI}$ & p4y & 2 & D-287589 & b & CAN & 52.00 & 13.40 & 13.90 & nd & 5.26 & 9.32 & 2.86 & .77 & .00 & 3.51 & .44 & .18 & nd & 101.64 \\
\hline DS 14 & $\mathrm{KI}$ & p4o & 2 & D-287575 & b & CAN & 51.00 & 13.50 & 12.70 & nd & 7.12 & 11.20 & 2.21 & .45 & .00 & 2.59 & .26 & .18 & nd & 101.21 \\
\hline DS-15 & $\mathrm{KI}$ & p3 & 2 & D-287560 & b & CAN & 50.90 & 13.50 & 12.60 & nd & 7.20 & 11.20 & 2.22 & .43 & .00 & 2.67 & .26 & .17 & nd & 101.15 \\
\hline DS-16 & $\mathrm{K}]$ & p2 & 2 & D-287585 & b & CAN & 50.80 & 13.50 & 12.70 & nd & 7.36 & 10.90 & 2.16 & .41 & .05 & 2.70 & .26 & .17 & nd & 101.01 \\
\hline DS- 17 & $\mathrm{KI}$ & $\mathrm{p} 4 \mathrm{o}$ & 2 & D-287584 & $\mathrm{b}$ & CAN & 51.10 & 13.70 & 12.40 & nd & 7.61 & 11.10 & 2.28 & .41 & .08 & 2.47 & .25 & .17 & nd & 101.57 \\
\hline DS-18 & $\mathrm{KI}$ & p2 & 2 & D-287576 & b & CAN & 49.80 & 12.00 & 12.70 & nd & 11.70 & 10.10 & 1.91 & .32 & .00 & 2.31 & .21 & .17 & nd & 101.22 \\
\hline DS-19 & $\mathrm{KI}$ & p4y & 2 & D-287583 & b & CAN & 50.80 & 13.80 & 12.50 & nd & 7.04 & 11.20 & 2.30 & .43 & .00 & 2.62 & .26 & .17 & nd & 101.12 \\
\hline DS-20 & $\mathrm{KI}$ & p4o & 2 & D-287582 & $\mathrm{b}$ & CAN & 50.70 & 13.10 & 12.10 & nd & 9.39 & 10.90 & 2.22 & .42 & .00 & 2.37 & .24 & .17 & nd & 101.61 \\
\hline DS-21 & $\mathrm{K}$ & p4o & 2 & D-287586 & b & CAN & 51.00 & 13.50 & 12.60 & nd & 6.87 & 11.30 & 2.21 & .44 & .09 & 2.60 & .26 & .18 & nd & 101.05 \\
\hline DS-22 & $\mathrm{KI}$ & p2 & 2 & D-287581 & b & CAN & 50.90 & 13.20 & 12.80 & nd & 7.58 & 11.30 & 2.16 & .44 & .04 & 2.67 & .26 & .18 & nd & 101.53 \\
\hline DS-23 & $\mathrm{KI}$ & p2 & 2 & D-284993 & $\mathrm{b}$ & CAN & 50.40 & 12.80 & 13.00 & nd & 8.38 & 11.20 & 2.13 & .43 & .00 & 2.69 & .26 & .18 & nd & 101.47 \\
\hline DS-25 & KI & p4o & 2 & D-287591 & b & CAN & 50.90 & 13.20 & 12.90 & nd & 7.76 & 11.00 & 2.13 & .43 & .00 & 2.59 & .26 & .18 & nd & 101.35 \\
\hline DS-26 & $\mathrm{KI}$ & p4y & 2 & D-315386 & b & CAN & 53.30 & 13.80 & 11.90 & nd & 5.46 & 9.45 & 2.90 & .83 & .00 & 2.70 & .39 & .16 & nd & 100.89 \\
\hline DS-28 & $\mathrm{K}]$ & p3 & 2 & D-284988 & b & CAN & 49.90 & 12.00 & 12.60 & nd & 12.30 & 9.75 & 2.01 & .36 & .00 & 2.32 & .23 & .17 & nd & 101.64 \\
\hline DS-29 & $\mathrm{KI}$ & p4o & 2 & D-287592 & $\mathrm{b}$ & CAN & 47.60 & 9.33 & 13.30 & nd & 19.30 & 7.80 & 1.53 & .29 & .00 & 1.72 & .18 & .18 & nd & 101.23 \\
\hline DS-30 & KI & pc4o & 2 & D-315385 & b & CAN & 51.50 & 13.40 & 13.90 & nd & 5.31 & 9.30 & 2.80 & .76 & .00 & 3.56 & .45 & .18 & nd & 101.16 \\
\hline DS-31 & $\mathrm{Kl}$ & p3 & 2 & D-287556 & b & CAN & 49.80 & 12.10 & 12.70 & nd & 11.50 & 10.30 & 1.92 & .34 & .00 & 2.26 & .21 & .17 & nd & 101.30 \\
\hline DS-32 & KI & p3 & 2 & D-284989 & b & CAN & 49.30 & 13.70 & 13.20 & nd & 6.93 & 11.00 & 2.46 & .70 & .00 & 3.32 & .36 & .18 & nd & 101.15 \\
\hline DS-33 & KI & p4y & 2 & D-315383 & b & CAN & 53.10 & 13.20 & 13.70 & nd & 4.09 & 8.00 & 3.24 & 1.11 & .00 & 3.58 & .57 & .18 & nd & 100.77 \\
\hline DS-34 & KI & pc4o & 2 & D-315384 & b & CAN & 51.40 & 13.30 & 13.90 & nd & 5.23 & 9.28 & 2.78 & .75 & .00 & 3.54 & .45 & .18 & nd & 100.81 \\
\hline DS-35 & $\mathrm{K}]$ & $\mathrm{p} 4 \mathrm{y}$ & 2 & D-314261 & b & CAN & 53.50 & 13.20 & 13.70 & nd & 4.12 & 7.92 & 3.17 & 1.12 & .00 & 3.52 & .59 & .18 & nd & 101.02 \\
\hline DS-36 & $\mathrm{KI}$ & $\mathrm{p} 4 \mathrm{y}$ & 2 & D-315382 & b & CAN & 51.40 & 12.70 & 14.40 & nd & 5.50 & 8.96 & 2.61 & .73 & .12 & 3.63 & .43 & .18 & nd & 100.66 \\
\hline DS-37 & KI & pc4o & 2 & D-284976 & b & CAN & 51.60 & 13.30 & 13.90 & nd & 5.18 & 9.23 & 2.78 & .76 & .00 & 3.52 & .46 & .19 & nd & 100.92 \\
\hline DS-39 & $\mathrm{KI}$ & $\mathrm{pc} 4 \mathrm{y}$ & 2 & D-315380 & b & CAN & 51.40 & 13.40 & 13.90 & nd & 5.46 & 9.23 & 2.65 & .72 & .00 & 3.47 & .43 & .18 & nd & 100.84 \\
\hline DS -40 & $\mathrm{KI}$ & $\mathrm{p} 4 \mathrm{y}$ & 2 & D-315379 & $\mathrm{b}$ & CAN & 51.10 & 13.40 & 12.50 & nd & 7.82 & 11.10 & 2.23 & .39 & .00 & 2.45 & .24 & .17 & nd & 101.40 \\
\hline
\end{tabular}


Table 1.--Chemical analyses-Continued

\begin{tabular}{|c|c|c|c|c|c|c|c|c|c|c|c|c|c|c|c|c|c|c|c|c|}
\hline $\begin{array}{c}\text { Sample } \\
\text { locality } \\
\text { No. }\end{array}$ & $\begin{array}{l}\text { Vol- } \\
\text { cano }\end{array}$ & Unit & $\begin{array}{c}\text { Map } \\
\text { sheet } \\
\text { No. }\end{array}$ & $\begin{array}{l}\text { Laboratory } \\
\text { No. }\end{array}$ & Remark & Collector & $\mathrm{SiO}_{2}$ & $\mathrm{Al}_{2} \mathrm{O}_{3}$ & $\mathrm{Fe}_{2} \mathrm{O}_{3}$ & $\mathrm{FeO}$ & $\mathrm{MgO}$ & $\mathrm{CaO}$ & $\mathrm{Na}_{2} \mathrm{O}$ & $\mathrm{K}_{2} \mathrm{O}$ & $\mathrm{H}_{2} \mathrm{O}$ & $\mathrm{TiO}_{2}$ & $\mathrm{P}_{2} \mathrm{O}_{5}$ & $\mathrm{MnO}$ & $\mathrm{CO}_{2}$ & Total \\
\hline DS-42 & $\mathrm{KI}$ & p2 & 2 & D-284987 & $\bar{b}$ & CAN & 50.80 & 13.50 & 12.70 & nd & 7.20 & 11.50 & 2.24 & .45 & .00 & 2.73 & .27 & .17 & nd & 101.56 \\
\hline DS-43 & $\mathrm{KI}$ & $\mathrm{pc40}$ & 2 & D-315377 & b & CAN & 51.70 & 13.20 & 14.10 & nd & 4.93 & 8.89 & 2.89 & .86 & .00 & 3.83 & .51 & .18 & nd & 101.09 \\
\hline DS-44 & $\mathrm{KI}$ & p4o & 2 & D-284990 & b & CAN & 51.30 & 13.70 & 12.50 & nd & 7.62 & 11.10 & 2.25 & .36 & .00 & 2.38 & .24 & .17 & nd & 101.62 \\
\hline DS-45 & KI & p3 & 2 & D-287555 & b & CAN & 50.40 & 13.10 & 12.80 & nd & 8.39 & 11.00 & 2.14 & .40 & .00 & 2.52 & .25 & .17 & nd & 101.17 \\
\hline DS-47 & $\mathrm{KI}$ & p3 & 2 & D-284991 & $b$ & CAN & 51.10 & 13.50 & 12.70 & nd & 7.07 & 11.50 & 2.30 & .42 & .00 & 2.70 & .27 & .18 & nd & 101.74 \\
\hline DS-48 & $\mathrm{KI}$ & p3 & 2 & D-284992 & $b$ & CAN & 50.70 & 13.40 & 12.80 & nd & 7.11 & 11.50 & 2.25 & .44 & .00 & 2.73 & .28 & .18 & nd & 101.39 \\
\hline DS-49 & $\mathrm{KI}$ & p40 & 2 & D-276901 & $b$ & CAN & 50.10 & 12.70 & 13.50 & nd & 7.99 & 10.90 & 2.23 & .50 & .00 & 2.85 & .29 & .18 & nd & 101.24 \\
\hline DS- 50 & $\mathrm{Kl}$ & p4o & 2 & D-276908 & $\mathrm{b}$ & CAN & 51.10 & 13.80 & 12.30 & nd & 7.26 & 11.00 & 2.14 & .35 & .04 & 2.39 & .24 & .17 & nd & 100.79 \\
\hline DS-51 & $\mathrm{KI}$ & p4y & 2 & D-314258 & b & CAN & 51.40 & 13.80 & 12.40 & nd & 6.63 & 10.70 & 2.44 & .54 & .00 & 2.68 & .30 & .17 & nd & 101.06 \\
\hline DS-52 & $\mathrm{KI}$ & p3 & 2 & D-276906 & $\mathrm{b}$ & CAN & 50.90 & 13.70 & 12.30 & nd & 7.29 & 11.30 & 2.24 & .40 & .00 & 2.52 & .24 & .17 & nd & 101.06 \\
\hline DS-53 & $\mathrm{KI}$ & p4o & 2 & D-276899 & b & CAN & 51.00 & 13.70 & 12.30 & nd & 7.49 & 11.20 & 2.24 & .41 & .00 & 2.47 & .25 & .17 & nd & 101.23 \\
\hline DS-54 & $\mathrm{KI}$ & p4o & 2 & D-276907 & $\mathrm{b}$ & CAN & 51.40 & 13.80 & 12.30 & nd & 6.79 & 11.10 & 2.40 & .37 & .00 & 2.48 & .25 & .17 & nd & 101.06 \\
\hline DS-55 & $\mathrm{KI}$ & p3 & 2 & D-284986 & $b$ & CAN & 50.70 & 13.40 & 12.60 & nd & 7.35 & 11.50 & 2.21 & .43 & .00 & 2.68 & .26 & .18 & nd & 101.31 \\
\hline DS-56 & KI & p3 & 2 & D-287570 & b & CAN & 50.80 & 13.30 & 12.70 & nd & 7.18 & 11.40 & 2.25 & .45 & .00 & 2.71 & .26 & .17 & nd & 101.22 \\
\hline DS-57 & $\mathrm{K}$ & p4y & 2 & D-284975 & b & CAN & 50.70 & 12.90 & 12.80 & nd & 8.94 & 10.70 & 2.12 & .38 & .00 & 2.44 & .25 & .18 & nd & 101.41 \\
\hline DS-58 & $\mathrm{KI}$ & p4o & 2 & D-276896 & b & CAN & 50.60 & 13.50 & 12.10 & nd & 7.63 & 11.00 & 2.26 & .43 & .14 & 2.47 & .26 & .17 & nd & 100.56 \\
\hline DS-59 & $\mathrm{KI}$ & p4o & 2 & D-278798 & b & CAN & 51.00 & 13.70 & 12.30 & nd & 7.03 & 11.20 & 2.16 & .38 & .00 & 2.38 & .24 & .17 & nd & 100.56 \\
\hline DS-60 & $\mathrm{Kl}$ & p4o & 2 & D-287571 & b & CAN & 51.30 & 13.80 & 12.20 & nd & 7.15 & 11.10 & 2.22 & .38 & .00 & 2.37 & .25 & .17 & nd & 100.94 \\
\hline DS-61 & $\mathrm{KI}$ & p4o & 2 & D-276889 & b & CAN & 51.30 & 13.80 & 12.50 & nd & 6.87 & 11.20 & 2.40 & .43 & .00 & 2.62 & .27 & .17 & nd & 101.56 \\
\hline DS-62 & $\mathrm{K} \mathbf{I}$ & p4o & 2 & D-287558 & b & CAN & 51.50 & 13.90 & 12.30 & nd & 7.03 & 11.10 & 2.21 & .39 & .02 & 2.42 & .25 & .17 & nd & 101.29 \\
\hline DS-63 & $\mathrm{KI}$ & $\mathrm{p} 4 \mathrm{y}$ & 2 & D-276893 & b & CAN & 50.50 & 13.20 & 12.50 & nd & 7.82 & 11.40 & 2.21 & .40 & .00 & 2.49 & .24 & .17 & nd & 100.93 \\
\hline DS-64 & $\mathrm{KI}$ & p4o & 2 & D-276892 & b & CAN & 49.80 & 13.20 & 12.40 & nd & 8.04 & 11.70 & 2.27 & .49 & .00 & 2.79 & .28 & .17 & nd & 101.14 \\
\hline DS-65 & $\mathrm{KI}$ & p4y & 2 & D-284974 & b & CAN & 50.20 & 12.70 & 12.80 & nd & 8.84 & 10.40 & 2.08 & .38 & .29 & 2.43 & .24 & .18 & nd & 100.54 \\
\hline DS-66 & $\mathrm{KI}$ & p4o & 2 & D-276890 & b & $\mathrm{CAN}$ & 50.90 & 13.40 & 12.30 & nd & 8.40 & 11.10 & 2.21 & .34 & .00 & 2.21 & .22 & .17 & nd & 101.25 \\
\hline DS-67 & $\mathrm{KI}$ & p4y & 2 & D-276891 & b & CAN & 50.20 & 13.10 & 12.60 & nd & 8.28 & 11.20 & 2.16 & .38 & .00 & 2.46 & .25 & .17 & nd & 100.80 \\
\hline DS-68 & $\mathrm{K}]$ & p3 & 2 & D-284985 & b & CAN & 50.60 & 12.50 & 12.40 & nd & 10.30 & 10.30 & 2.09 & .32 & .00 & 2.26 & .23 & .17 & nd & 101.17 \\
\hline DS-69 & KI & p5-1919 & 2 & D-276894 & b & CAN & 49.90 & 13.10 & 12.50 & nd & 8.50 & 11.60 & 2.20 & .48 & .00 & 2.72 & .28 & .17 & nd & 101.45 \\
\hline DS-70 & $\mathrm{KI}$ & $\mathrm{p} 4 \mathrm{y}$ & 2 & D-284973 & b & CAN & 50.80 & 13.20 & 12.70 & nd & 8.37 & 10.80 & 2.11 & .38 & .00 & 2.47 & .25 & .17 & nd & 101.25 \\
\hline DS-71 & $\mathrm{KI}$ & p4o & 2 & D-284961 & b & CAN & 50.90 & 13.70 & 12.30 & nd & 7.19 & 11.00 & 2.29 & .44 & .00 & 2.56 & .27 & .17 & nd & 100.82 \\
\hline DS-72 & $\mathrm{KI}$ & p4y & 2 & D-287593 & b & CAN & 50.30 & 11.90 & 13.10 & nd & 11.30 & 10.00 & 1.92 & .38 & .00 & 2.38 & .25 & .18 & nd & 101.71 \\
\hline DS-73 & $\mathrm{Kl}$ & p4y & 2 & D-284962 & b & CAN & 51.10 & 13.80 & 12.70 & nd & 6.54 & 10.80 & 2.39 & .49 & .07 & 2.89 & .30 & .18 & nd & 101.26 \\
\hline DS-74 & $\mathrm{KI}$ & p4o & 2 & D-284967 & b & CAN & 51.10 & 13.70 & 12.20 & nd & 7.18 & 11.00 & 2.26 & .40 & .00 & 2.40 & .25 & .17 & nd & 100.66 \\
\hline DT-2 & KI & p4o & 2 & D-319081 & $\mathrm{b}$ & RTH & 50.40 & 13.00 & 12.70 & nd & 8.70 & 10.90 & 2.14 & .42 & .00 & 2.49 & .27 & .17 & nd & 101.19 \\
\hline DT-3 & $\mathrm{KI}$ & p2 & 2 & D-319079 & $b$ & $\mathrm{RTH}$ & 48.50 & 10.70 & 13.10 & nd & 14.90 & 9.18 & 1.87 & .34 & .00 & 2.02 & .23 & .17 & nd & 101.01 \\
\hline DT-4 & KI & p4o & 2 & D-319088 & b & RTH & 51.00 & 13.50 & 12.60 & nd & 7.32 & 11.50 & 2.28 & .42 & .00 & 2.49 & .26 & .17 & nd & 101.54 \\
\hline DT-5 & $\mathrm{KI}$ & p4o & 2 & D-319087 & b & $\mathrm{RTH}$ & 50.80 & 13.00 & 12.60 & nd & 8.75 & 10.80 & 2.15 & .40 & .00 & 2.49 & .26 & .17 & nd & 101.42 \\
\hline DT-6 & $\mathrm{KI}$ & p2 & 2 & D-319080 & b & $\mathrm{RTH}$ & 49.50 & 12.50 & 12.80 & nd & 10.80 & 10.30 & 2.03 & .37 & .01 & 2.35 & .26 & .17 & nd & 101.09 \\
\hline DT-7 & $\mathrm{KI}$ & $\mathrm{p} 4 \mathrm{o}$ & 2 & D-319086 & b & RTH & 50.00 & 13.00 & 12.60 & nd & 8.46 & 11.30 & 2.26 & .47 & .03 & 2.63 & .28 & .17 & nd & 101.20 \\
\hline DT-8 & $\mathrm{KI}$ & p4 & 2 & D-319094 & b & RTH & 50.50 & 13.30 & 12.60 & nd & 7.98 & 11.20 & 2.13 & .38 & .21 & 2.49 & .25 & .17 & nd & 101.21 \\
\hline DT-9 & $\mathrm{KI}$ & p4 & 2 & D-319093 & $b$ & RTH & 50.40 & 13.20 & 12.70 & nd & 8.47 & 11.20 & 2.16 & .36 & .00 & 2.46 & .25 & .18 & nd & 101.38 \\
\hline
\end{tabular}


Table 1.-Chemical analyses-Continued

\begin{tabular}{|c|c|c|c|c|c|c|c|c|c|c|c|c|c|c|c|c|c|c|c|c|}
\hline $\begin{array}{c}\text { Sample } \\
\text { locality } \\
\text { No. }\end{array}$ & $\begin{array}{l}\text { Vol- } \\
\text { cano }\end{array}$ & Unit & $\begin{array}{c}\text { Map } \\
\text { sheet } \\
\text { No. }\end{array}$ & $\begin{array}{l}\text { Laboratory } \\
\text { No. }\end{array}$ & Remark & Collector & $\mathrm{SiO}_{2}$ & $\mathrm{Al}_{2} \mathrm{O}_{3}$ & $\mathrm{Fe}_{2} \mathrm{O}_{3}$ & $\mathrm{FeO}$ & $\mathrm{MgO}$ & $\mathrm{CaO}$ & $\mathrm{Na}_{2} \mathrm{O}$ & $\mathrm{K}_{2} \mathrm{O}$ & $\mathrm{H}_{2} \mathrm{O}$ & $\mathrm{TiO}_{2}$ & $\mathrm{P}_{2} \mathrm{O}_{5}$ & $\mathrm{MnO}$ & $\mathrm{CO}_{2}$ & Total \\
\hline DT-10 & $\mathrm{KI}$ & p4o & 2 & D-319095 & $\bar{b}$ & RTH & 50.40 & 13.00 & 12.70 & nd & 8.82 & 10.90 & 2.16 & .41 & .00 & 2.46 & .26 & .17 & nd & 101.28 \\
\hline DT- 12 & KI & p3 & 2 & D-319131 & b & RTH & 50.70 & 13.40 & 12.50 & nd & 7.46 & 11.60 & 2.20 & .43 & .00 & 2.62 & .28 & .17 & nd & 101.36 \\
\hline DT-13 & $\mathrm{KI}$ & p2 & 2 & D-319130 & $\mathrm{b}$ & RTH & 50.80 & 13.30 & 12.40 & nd & 8.34 & 10.90 & 2.26 & .41 & .00 & 2.49 & .26 & .17 & nd & 101.33 \\
\hline DT-14 & $\mathrm{KI}$ & p4o & 2 & D-319124 & b & RTH & 51.40 & 13.90 & 12.20 & nd & 7.10 & 11.10 & 2.32 & .39 & .00 & 2.38 & .26 & .17 & nd & 101.22 \\
\hline DT-15 & $\mathrm{K}$ & p2 & 2 & D-319123 & b & RTH & 49.90 & 13.20 & 12.70 & nd & 8.31 & 10.80 & 2.18 & .42 & .18 & 2.61 & .28 & .17 & nd & 100.75 \\
\hline DT-16 & $\mathrm{KI}$ & p2 & 2 & D-319125 & $\mathrm{b}$ & RTH & 50.10 & 13.30 & 12.50 & nd & 7.96 & 11.00 & 2.24 & .46 & .10 & 2.72 & .29 & .17 & nd & 100.84 \\
\hline DT-17 & $\mathrm{KI}$ & p3 & 2 & D-319122 & b & RTH & 49.60 & 12.60 & 12.80 & nd & 9.73 & 10.70 & 2.11 & .43 & .00 & 2.57 & .28 & .17 & nd & 100.99 \\
\hline DT-18 & KI & p4 & 2 & D-319121 & b & RTH & 50.30 & 12.80 & 12.70 & nd & 9.44 & 10.90 & 2.12 & .39 & .00 & 2.41 & .24 & .17 & nd & 101.47 \\
\hline DT-19 & $\mathrm{Kl}$ & p4o & 2 & D-319089 & b & RTH & 51.40 & 13.40 & 12.80 & nd & 6.87 & 11.00 & 2.22 & .45 & .00 & 2.75 & .27 & .17 & nd & 101.33 \\
\hline DT-20 & KI & p3 & 2 & D-319127 & $\mathrm{b}$ & RTH & 49.50 & 12.90 & 12.60 & nd & 9.19 & 10.60 & 2.19 & .45 & .05 & 2.59 & .29 & .17 & nd & 100.53 \\
\hline DT-21 & $\mathrm{KI}$ & p4 & 2 & D-319092 & $\mathrm{b}$ & RTH & 49.80 & 13.20 & 12.60 & nd & 7.96 & 11.50 & 2.12 & .43 & .15 & 2.67 & .27 & .17 & nd & 100.87 \\
\hline DT-22 & $\mathrm{KI}$ & p4 & 2 & D-319090 & b & RTH & 50.80 & 13.20 & 12.70 & nd & 7.92 & 11.30 & 2.12 & .39 & .00 & 2.54 & .25 & .17 & nd & 101.39 \\
\hline DT-23 & $\mathrm{KI}$ & p3 & 2 & D-319126 & $\mathrm{b}$ & RTH & 50.60 & 13.40 & 12.50 & nd & 7.22 & 11.40 & 2.18 & .44 & .09 & 2.66 & .28 & .17 & nd & 100.94 \\
\hline DT-24 & KI & p4 & 2 & D-319091 & b & RTH & 51.00 & 13.40 & 12.40 & nd & 7.81 & 11.50 & 2.24 & .38 & .00 & 2.52 & .25 & .17 & nd & 101.67 \\
\hline DT-25 & $\mathrm{KI}$ & p4o & 2 & D-319129 & b & RTH & 51.50 & 13.90 & 12.10 & nd & 6.94 & 11.20 & 2.30 & .41 & .00 & 2.42 & .26 & .16 & nd & 101.19 \\
\hline DT-26 & KI & $\mathrm{p} 3$ & 2 & D-319128 & $\mathrm{b}$ & RTH & 51.10 & 13.40 & 12.40 & nd & 7.15 & 11.50 & 2.24 & .44 & .00 & 2.64 & .26 & .17 & nd & 101.30 \\
\hline DT-27 & $\mathrm{KI}$ & p4o & 2 & D-319114 & $\mathrm{b}$ & RTH & 50.60 & 13.20 & 12.50 & nd & 8.33 & 10.80 & 2.14 & .41 & .07 & 2.48 & .26 & .17 & nd & 100.96 \\
\hline DT-28 & $\mathrm{KI}$ & $\mathrm{p} 4 \mathrm{o}$ & 2 & D-319115 & $b$ & RTH & 50.70 & 13.40 & 12.80 & nd & 7.87 & 11.20 & 2.20 & .42 & .00 & 2.46 & .25 & .17 & nd & 101.47 \\
\hline DT-29 & KI & p3 & 2 & D-319120 & $\mathrm{b}$ & RTH & 50.10 & 13.60 & 12.40 & nd & 7.11 & 10.90 & 2.12 & .42 & 1.08 & 2.66 & .27 & .16 & nd & 100.82 \\
\hline DT-31 & $\mathrm{KI}$ & p4 & 2 & D-319119 & $\mathrm{b}$ & RTH & 50.90 & 13.30 & 12.20 & nd & 8.65 & 11.00 & 2.12 & .36 & .00 & 2.37 & .23 & .17 & nd & 101.30 \\
\hline DT-32 & $\mathrm{KI}$ & p3 & 2 & D-319118 & b & RTH & 50.80 & 13.40 & 12.50 & nd & 7.19 & 11.50 & 2.24 & .44 & .00 & 2.66 & .26 & .17 & nd & 101.16 \\
\hline DT-33 & KI & p4o & 2 & D-319113 & $\mathrm{b}$ & RTH & 51.10 & 13.40 & 12.10 & nd & 7.59 & 11.60 & 2.21 & .39 & .00 & 2.55 & .25 & .17 & nd & 101.36 \\
\hline DT-34 & $\mathrm{Kl}$ & p4 & 2 & D-319082 & $\mathrm{b}$ & RTH & 50.90 & 13.50 & 12.40 & nd & 7.50 & 11.60 & 2.12 & .37 & .00 & 2.52 & .25 & .17 & nd & 101.33 \\
\hline DT-35 & $\mathrm{KI}$ & p4y & 2 & D-319083 & $\mathrm{b}$ & RTH & 51.00 & 13.10 & 12.60 & nd & 8.28 & 11.00 & 2.21 & .42 & .00 & 2.59 & .27 & .17 & nd & 101.64 \\
\hline DT-36 & $\mathrm{KI}$ & $\mathrm{p4}$ & 2 & D-319084 & b & RTH & 49.90 & 12.00 & 12.40 & nd & 11.70 & 10.20 & 1.93 & .38 & .00 & 2.35 & .24 & .17 & nd & 101.27 \\
\hline DT-37 & $\mathrm{KI}$ & p4 & 2 & D-319085 & $\mathrm{b}$ & RTH & 47.40 & 13.20 & 12.90 & nd & 9.03 & 9.30 & 1.71 & .33 & 4.41 & 2.49 & .23 & .17 & nd & 101.17 \\
\hline DT-38 & $\mathrm{KI}$ & p4 & 2 & D-319117 & $\mathrm{b}$ & RTH & 49.90 & 12.20 & 12.80 & nd & 11.20 & 10.20 & 1.99 & .33 & .00 & 2.19 & .22 & .17 & nd & 101.20 \\
\hline DT-39 & $\mathrm{KI}$ & $\mathrm{p} 4$ & 2 & D-319097 & $\mathrm{b}$ & RTH & 47.80 & 9.86 & 13.20 & nd & 17.80 & 8.23 & 1.72 & .32 & .00 & 1.85 & .20 & .17 & nd & 101.15 \\
\hline DT-40 & $\mathrm{KI}$ & p4 & 2 & D-319099 & b & RTH & 50.70 & 13.10 & 12.60 & nd & 8.14 & 10.90 & 2.22 & .42 & .11 & 2.56 & .27 & .17 & nd & 101.19 \\
\hline DT-41 & $\mathrm{KI}$ & p4 & 2 & D-319100 & b & RTH & 50.50 & 12.80 & 12.60 & nd & 8.59 & 10.80 & 2.17 & .42 & .11 & 2.55 & .27 & .17 & nd & 100.98 \\
\hline DT-42 & $\mathrm{K}$ & p4 & 2 & D-319116 & b & RTH & 50.50 & 12.70 & 12.50 & nd & 9.72 & 10.70 & 2.07 & .34 & .00 & 2.32 & .22 & .17 & nd & 101.24 \\
\hline DT-43 & $\mathrm{K} J$ & p4 & 2 & D-319107 & b & RTH & 51.20 & 13.50 & 12.10 & nd & 7.72 & 11.30 & 2.17 & .36 & .00 & 2.45 & .24 & .16 & nd & 101.20 \\
\hline DT-47 & $\mathrm{Kl}$ & $\mathrm{p} 40$ & 2 & D-319074 & b & RTH & 49.70 & 12.10 & 12.80 & nd & 11.60 & 9.99 & 2.04 & .34 & .00 & 2.21 & .23 & .17 & nd & 101.18 \\
\hline DT-48 & $\mathrm{KI}$ & p4o & 2 & D-319104 & $b$ & RTH & 51.60 & 13.80 & 12.20 & nd & 7.19 & 11.20 & 2.34 & .40 & .00 & 2.39 & .25 & .17 & nd & 101.54 \\
\hline DT -53 & $\mathrm{Kl}$ & p4 & 2 & D-319112 & b & RTH & 48.10 & 11.00 & 13.10 & nd & 14.30 & 9.03 & 1.78 & .34 & .30 & 2.06 & .21 & .17 & nd & 100.39 \\
\hline DT-54 & $\mathrm{Kl}$ & $\mathrm{p} 40$ & 2 & D-319111 & b & $\mathrm{RTH}$ & 50.00 & 12.20 & 12.70 & nd & 11.40 & 9.95 & 2.01 & .32 & .00 & 2.09 & .21 & .17 & nd & 101.05 \\
\hline DU-1 & $\mathrm{KI}$ & p4o & 2 & D-291730 & b & RWH & 50.70 & 13.20 & 12.90 & nd & 7.69 & 11.30 & 2.20 & .42 & .00 & 2.53 & .25 & .18 & nd & 101.37 \\
\hline DU-2 & $\mathrm{KI}$ & p4o & 2 & D-291731 & b & RWH & 50.60 & 13.30 & 12.90 & nd & 7.66 & 11.30 & 2.17 & .42 & .00 & 2.49 & .25 & .18 & nd & 101.27 \\
\hline DU-3 & KI & p4o & 2 & D-291729 & b & RWH & 50.90 & 13.40 & 12.80 & nd & 7.37 & 11.50 & 2.14 & .42 & .00 & 2.55 & .25 & .17 & nd & 101.50 \\
\hline DU-4 & KI & $\mathrm{p} 40$ & 2 & D-291732 & $\mathrm{b}$ & RWH & 50.60 & 13.30 & 12.80 & nd & 7.57 & 11.30 & 2.17 & .42 & .00 & 2.55 & .25 & .18 & nd & 101.14 \\
\hline
\end{tabular}


Table 1.-Chemical analyses-Continued

\begin{tabular}{|c|c|c|c|c|c|c|c|c|c|c|c|c|c|c|c|c|c|c|c|c|}
\hline $\begin{array}{c}\text { Sample } \\
\text { locality } \\
\text { No. }\end{array}$ & $\begin{array}{l}\text { Vol- } \\
\text { cano }\end{array}$ & Unit & $\begin{array}{l}\text { Map } \\
\text { sheet } \\
\text { No. }\end{array}$ & $\begin{array}{c}\text { Laboratory } \\
\text { No. }\end{array}$ & Remark & Collector & $\mathrm{SiO}_{2}$ & $\mathrm{Al}_{2} \mathrm{O}_{3}$ & $\mathrm{Fe}_{2} \mathrm{O}_{3}$ & $\mathrm{FeO}$ & $\mathrm{MgO}$ & $\mathrm{CaO}$ & $\mathrm{Na}_{2} \mathrm{O}$ & $\mathrm{K}_{2} \mathrm{O}$ & $\mathrm{H}_{2} \mathrm{O}$ & $\mathrm{TiO}_{2}$ & $\mathrm{P}_{2} \mathrm{O}_{5}$ & $\mathrm{MnO}$ & $\mathrm{CO}_{2}$ & Total \\
\hline DU-5 & KI & $\mathrm{p} 40$ & 2 & D-291733 & $\mathrm{b}$ & RWH & 50.80 & 13.90 & 12.40 & nd & 7.11 & 11.50 & 2.18 & .42 & .00 & 2.52 & .25 & .17 & nd & 101.25 \\
\hline DU-6 & $\mathrm{KI}$ & p4o & 2 & D-291737 & b & RWH & 48.20 & 13.10 & 13.10 & nd & 8.03 & 10.90 & 2.38 & .90 & .25 & 3.30 & .41 & .17 & nd & 100.74 \\
\hline DU-10 & KI & p4o & 2 & D-291741 & b & RWH & 50.40 & 13.30 & 12.80 & nd & 7.65 & 11.20 & 2.17 & .42 & .03 & 2.50 & .25 & .18 & nd & 100.90 \\
\hline DU-11 & $\mathrm{KI}$ & p4o & 2 & D-291740 & b & RWH & 50.60 & 13.30 & 12.60 & nd & 7.50 & 11.30 & 2.14 & .42 & .00 & 2.49 & .25 & .17 & nd & 100.77 \\
\hline DU-12 & $\mathrm{KI}$ & p4o & 2 & D-291738 & $b$ & RWH & 50.50 & 13.30 & 12.70 & nd & 7.67 & 11.30 & 2.13 & .42 & .04 & 2.49 & .25 & .17 & nd & 100.97 \\
\hline DU-13 & $\mathrm{KI}$ & p4o & 2 & D-291739 & b & RWH & 50.30 & 13.40 & 12.60 & nd & 7.60 & 11.30 & 2.13 & .42 & .25 & 2.48 & .25 & .17 & nd & 100.90 \\
\hline DU-17 & KI & p4y & 2 & D-291788 & $\mathrm{b}$ & RWH & 51.30 & 13.30 & 13.30 & nd & 5.64 & 9.64 & 2.71 & .71 & .00 & 3.41 & .41 & .18 & nd & 100.60 \\
\hline DU-18 & KI & p4y & 2 & D-291789 & b & RWH & 50.30 & 13.10 & 12.60 & nd & 8.15 & 11.10 & 2.16 & .43 & .12 & 2.53 & .26 & .17 & nd & 100.92 \\
\hline DU-19 & KI & p2 & 2 & D-291747 & b & RWH & 50.70 & 13.60 & 12.80 & nd & 7.05 & 10.80 & 2.44 & .54 & .00 & 2.87 & .30 & .17 & nd & 101.27 \\
\hline DU-24 & $\mathrm{KI}$ & p4o & 2 & D-291750 & b & RWH & 51.00 & 13.60 & 12.90 & nd & 6.37 & 10.30 & 2.51 & .60 & .00 & 3.06 & .34 & .17 & nd & 100.85 \\
\hline DU-26 & $\mathrm{KI}$ & p4y & 2 & D-291751 & b & $\mathrm{RWH}$ & 49.80 & 13.30 & 12.40 & nd & 8.31 & 11.10 & 2.13 & .44 & .17 & 2.68 & .25 & .17 & nd & 100.75 \\
\hline DU-27 & $\mathrm{KI}$ & p4o & 2 & D-291748 & b & RWH & 50.60 & 13.10 & 12.10 & nd & 8.21 & 11.30 & 2.12 & .40 & .00 & 2.42 & .25 & .17 & nd & 100.67 \\
\hline DU-28 & $\mathrm{KI}$ & p4y & 2 & D-291743 & b & RWH & 50.80 & 13.90 & 12.70 & nd & 6.53 & 10.70 & 2.48 & .56 & .00 & 2.95 & .31 & .17 & nd & 101.10 \\
\hline DU-30 & $\mathrm{KI}$ & p4y & 2 & D-291744 & b & RWH & 50.00 & 12.50 & 12.30 & nd & 9.97 & 10.60 & 2.04 & .39 & .36 & 2.44 & .24 & .17 & nd & 101.01 \\
\hline DU-31 & KI & $\mathrm{p} 2$ & 2 & D-291745 & b & RWH & 51.00 & 13.40 & 12.10 & nd & 7.43 & 11.40 & 2.19 & .43 & .00 & 2.69 & .27 & .16 & nd & 101.07 \\
\hline EN-1 & ML & k2 & 3 & D-313500 & b & JPL & 51.00 & 13.70 & 12.40 & nd & 7.35 & 10.70 & 2.24 & .37 & .43 & 2.19 & .26 & .17 & nd & 100.81 \\
\hline EN-2 & ML & k3 & 3 & D-313496 & b & JPL & 50.80 & 12.60 & 12.70 & nd & 9.94 & 10.10 & 2.09 & .38 & .00 & 2.17 & .25 & .17 & nd & 101.20 \\
\hline EN-3 & ML & $k 2$ & 3 & D-313497 & $b$ & $\mathrm{JPL}$ & 50.70 & 12.90 & 12.50 & nd & 9.51 & 10.20 & 2.13 & .34 & .00 & 2.26 & .26 & .17 & nd & 100.97 \\
\hline EN-4 & ML & k1y & 3 & D-313498 & b & JPL & 51.30 & 13.40 & 12.30 & nd & 7.59 & 10.60 & 2.19 & .35 & .00 & 2.06 & .23 & .17 & nd & 100.19 \\
\hline EN-5 & $\mathrm{ML}$ & k4 & 3 & D-313499 & b & JPL & 51.20 & 13.60 & 12.10 & nd & 7.55 & 10.80 & 2.15 & .40 & .35 & 2.20 & .29 & .17 & nd & 100.81 \\
\hline EO-1 & ML & $k 2$ & 3 & D-313505 & b & JPL & 51.80 & 13.80 & 12.50 & nd & 6.79 & 10.80 & 2.28 & .41 & .00 & 2.20 & .26 & .17 & nd & 101.01 \\
\hline EO-2 & ML & $k 2$ & 3 & D-313506 & b & JPL & 51.70 & 13.80 & 12.40 & nd & 6.82 & 10.80 & 2.30 & .41 & .00 & 2.19 & .26 & .17 & nd & 100.85 \\
\hline EO-3 & $\mathrm{ML}$ & k1y & 3 & D-313507 & b & JPL & 50.80 & 13.10 & 12.20 & nd & 9.13 & 10.20 & 2.00 & .41 & .35 & 2.10 & .26 & .16 & nd & 100.71 \\
\hline EO-4 & $\mathrm{ML}$ & k1y & 3 & D-313508 & b & JPL & 51.80 & 13.60 & 13.10 & nd & 6.27 & 10.50 & 2.33 & .43 & .00 & 2.42 & .28 & .18 & nd & 100.91 \\
\hline EO-5 & ML & $\mathrm{k} 3$ & 3 & D-313509 & b & $J P L$ & 52.00 & 13.70 & 12.00 & nd & 7.14 & 10.50 & 2.31 & .46 & .00 & 2.28 & .28 & .16 & nd & 100.83 \\
\hline EO-6 & ML & K3 & 3 & D-313510 & $b$ & JPL & 48.90 & 10.10 & 12.50 & nd & 17.30 & 8.20 & 1.68 & .30 & .00 & 1.78 & .19 & .17 & nd & 101.12 \\
\hline EO-7 & ML & $k 2$ & 3 & D-313512 & b & JPL & 51.30 & 13.30 & 12.80 & nd & 7.45 & 10.30 & 2.29 & .44 & .00 & 2.55 & .28 & .17 & nd & 100.88 \\
\hline EO-8 & ML & k4 & 3 & D-313511 & b & JPL & 51.80 & 13.70 & 11.80 & nd & 7.32 & 10.70 & 2.26 & .46 & .00 & 2.25 & .26 & .16 & nd & 100.71 \\
\hline EP-1 & ML & k5-1950 & 3 & ML-77 & a & JMR & 51.42 & 13.77 & 12.04 & nd & 6.75 & 10.56 & 2.11 & .38 & nd & 2.08 & .23 & .15 & nd & 99.49 \\
\hline EP-2 & ML & k5-1949 & 3 & ML-69 & a & JMR & 51.56 & 13.63 & 12.13 & nd & 6.92 & 10.52 & 2.21 & .39 & nd & 2.05 & .23 & .17 & nd & 99.81 \\
\hline EP-3 & ML & k4 & 3 & SW-63A & a & JMR & 52.15 & 14.05 & 11.74 & nd & 6.60 & 10.49 & 2.30 & .44 & nd & 2.16 & .28 & .16 & nd & 100.37 \\
\hline EQ-1 & ML & k3 & 3 & D-306040 & b & EWW & 48.90 & 10.10 & 12.80 & nd & 16.50 & 8.30 & 1.77 & .31 & .00 & 1.82 & .20 & .17 & nd & 100.87 \\
\hline EQ-2 & ML & $\mathrm{k} 3$ & 3 & NE-62 & a & JPL & 51.82 & 13.52 & 12.33 & nd & 6.27 & 10.58 & 2.45 & .42 & nd & 2.43 & .27 & .18 & nd & 100.27 \\
\hline EQ-3 & ML & $\mathrm{k} 3$ & 3 & D-306042 & b & EWW & 52.10 & 13.40 & 12.60 & nd & 6.22 & 10.50 & 2.41 & .50 & .00 & 2.65 & .31 & .18 & nd & 100.87 \\
\hline EQ-4 & ML & $\mathrm{k} 3$ & 3 & D-306041 & b & EWW & 50.80 & 12.30 & 12.20 & nd & 10.80 & 9.56 & 2.08 & .40 & .00 & 2.41 & .27 & .16 & nd & 100.98 \\
\hline EQ-5 & ML & k5-1949 & 3 & D-306034 & $b$ & EWW & 52.00 & 13.70 & 12.40 & nd & 7.16 & 10.90 & 2.31 & .37 & .00 & 2.10 & .24 & .17 & nd & 101.35 \\
\hline EQ-6 & ML & k3 & 3 & D-306036 & $\mathrm{b}$ & EWW & 51.90 & 13.70 & 12.20 & nd & 6.40 & 10.70 & 2.36 & .48 & .00 & 2.52 & .30 & .17 & nd & 100.73 \\
\hline EQ-7 & ML & k1y & 3 & NE-61 & a & JPL & 51.40 & 13.41 & 13.59 & nd & 6.01 & 9.76 & 2.78 & .47 & nd & 2.66 & .31 & .18 & nd & 100.57 \\
\hline EQ-8 & ML & $\mathrm{k} 3$ & 3 & D-306035 & b & EWW & 49.70 & 11.20 & 12.40 & nd & 14.10 & 8.88 & 1.79 & .31 & .04 & 1.98 & .21 & .17 & nd & 100.78 \\
\hline EQ-11 & ML & k5-1950 & 3 & ML-217 & a & JMR & 51.81 & 13.80 & 12.06 & nd & 6.42 & 10.40 & 2.25 & .43 & nd & 2.23 & .26 & .17 & nd & 99.83 \\
\hline
\end{tabular}


Table 1.-Chemical analyses-Continued

\begin{tabular}{|c|c|c|c|c|c|c|c|c|c|c|c|c|c|c|c|c|c|c|c|c|}
\hline $\begin{array}{l}\text { Sample } \\
\text { locality } \\
\text { No. }\end{array}$ & $\begin{array}{l}\text { Vol- } \\
\text { cano }\end{array}$ & Unit & $\begin{array}{c}\text { Map } \\
\text { sheet } \\
\text { No. }\end{array}$ & $\begin{array}{l}\text { Laboratory } \\
\text { No. }\end{array}$ & Remark & Collector & $\mathrm{SiO}_{2}$ & $\mathrm{Al}_{2} \mathrm{O}_{3}$ & $\mathrm{Fe}_{2} \mathrm{O}_{3}$ & $\mathrm{FeO}$ & $\mathrm{MgO}$ & $\mathrm{CaO}$ & $\mathrm{Na}_{2} \mathrm{O}$ & $\mathrm{K}_{2} \mathrm{O}$ & $\mathrm{H}_{2} \mathrm{O}$ & $\mathrm{TiO}_{2}$ & $\mathrm{P}_{2} \mathrm{O}_{5}$ & $\mathrm{MnO}$ & $\mathrm{CO}_{2}$ & Total \\
\hline EQ-12 & ML & k5-1926 & 3 & ML-73 & $\mathrm{a}$ & JMR & 51.71 & 13.67 & 12.09 & nd & 6.42 & 10.49 & 2.14 & .40 & nd & 2.20 & .25 & .16 & nd & 99.53 \\
\hline EQ-13 & ML & k5-1940 & 3 & ML-74 & a & JMR & 51.58 & 13.70 & 11.83 & nd & 7.03 & 10.47 & 2.22 & .46 & nd & 2.13 & .26 & .16 & nd & 99.84 \\
\hline EQ-14 & ML & k5-1975 & 3 & ML-78 & a & JMR & 51.54 & 13.73 & 12.22 & nd & 6.50 & 10.49 & 2.19 & .39 & nd & 2.09 & .22 & .16 & nd & 99.53 \\
\hline EQ-15 & ML & k5-1851 & 3 & ML-80 & a & JMR & 51.61 & 13.75 & 11.83 & nd & 6.85 & 10.15 & 2.15 & .45 & nd & 2.14 & .26 & .16 & nd & 99.35 \\
\hline EQ-16 & ML & k5-1949 & 3 & ML-81 & a & JMR & 51.55 & 13.62 & 12.10 & nd & 6.99 & 10.51 & 2.08 & .37 & nd & 2.05 & .22 & .16 & nd & 99.65 \\
\hline $\mathrm{EQ}-20$ & ML & k3 & 3 & D-306037 & b & EWW & 52.20 & 13.70 & 12.60 & nd & 6.53 & 10.60 & 2.42 & .49 & .00 & 2.36 & .31 & .17 & nd & 101.38 \\
\hline $\mathrm{EQ}-21$ & ML & k3 & 3 & D-306047 & b & EWW & 51.90 & 13.70 & 12.00 & nd & 6.83 & 10.80 & 2.36 & .45 & .00 & 2.41 & .28 & .17 & nd & 100.90 \\
\hline$E Q-23$ & ML & k5-1940 & 3 & ML-130 & a & JMR & 51.23 & 13.68 & 12.14 & nd & 6.96 & 10.64 & 2.19 & .37 & nd & 2.06 & .23 & .17 & nd & 99.67 \\
\hline $\mathrm{EQ}-25$ & ML & k3 & 3 & D-306038 & $b$ & EWW & 52.00 & 13.80 & 12.50 & nd & 6.47 & 10.50 & 2.38 & .49 & .00 & 2.35 & .30 & .17 & nd & 100.96 \\
\hline EQ-26 & ML & $\mathrm{k} 3$ & 3 & D-306043 & b & EWW & 52.00 & 13.60 & 12.20 & nd & 6.76 & 10.80 & 2.27 & .46 & .00 & 2.50 & .29 & .17 & nd & 101.05 \\
\hline EQ-28 & ML & k3 & 3 & D-306046 & b & EWW & 51.90 & 13.80 & 11.90 & nd & 6.64 & 10.80 & 2.37 & .43 & .00 & 2.47 & .28 & .17 & nd & 100.76 \\
\hline EQ-30 & $\mathrm{ML}$ & k3 & 3 & D-306045 & b & EWW & 50.40 & 11.90 & 12.10 & nd & 11.80 & 9.71 & 1.94 & .33 & .00 & 2.06 & .22 & .16 & nd & 100.62 \\
\hline EQ-31 & ML & k3 & 3 & D-306044 & b & EWW & 50.80 & 12.20 & 11.80 & nd & 11.20 & 9.62 & 2.09 & .40 & .00 & 2.35 & .26 & .16 & nd & 100.88 \\
\hline $\mathrm{EQ}-32$ & ML & k5-1880-81 & 3 & ML-41 & a & JMR & 50.54 & 12.02 & 12.43 & nd & 10.75 & 9.17 & 1.85 & .28 & nd & 1.75 & .17 & .17 & nd & 99.13 \\
\hline$E Q-33$ & ML & k4 & 3 & D-306039 & b & EWW & 51.90 & 13.80 & 12.10 & nd & 6.89 & 10.80 & 2.28 & .40 & .00 & 2.11 & .26 & .17 & nd & 100.71 \\
\hline$E Q-34$ & ML & K3 & 3 & D-306048 & b & EWW & 52.10 & 13.70 & 12.40 & nd & 6.37 & 10.60 & 2.38 & .51 & .00 & 2.54 & .31 & .17 & nd & 101.08 \\
\hline ER-1 & ML & $k 2$ & 3 & D-287083 & b & MDJ & 50.40 & 11.90 & 11.90 & nd & 12.60 & 9.38 & 1.91 & .31 & .00 & 2.04 & .21 & .16 & nd & 100.81 \\
\hline ER-2 & ML & $\mathrm{K} 3$ & 3 & D-313840 & b & JPL & 51.20 & 12.60 & 12.00 & nd & 10.20 & 9.85 & 2.16 & .40 & .00 & 2.42 & .26 & .16 & nd & 101.25 \\
\hline ER-3 & ML & $\mathrm{k} 3$ & 3 & D-287089 & b & MDJ & 51.90 & 13.60 & 12.40 & nd & 6.87 & 11.00 & 2.16 & .42 & .00 & 2.45 & .27 & .17 & nd & 101.24 \\
\hline ER-4 & ML & k1o & 3 & D-287104 & b & MDJ & 51.00 & 13.40 & 12.40 & nd & 8.47 & 10.40 & 2.06 & .36 & .00 & 2.03 & .23 & .17 & nd & 100.52 \\
\hline ER-5 & ML & k2 & 3 & D-287098 & b & MDJ & 50.70 & 12.40 & 12.30 & nd & 10.80 & 9.95 & 2.01 & .32 & .00 & 2.14 & .22 & .17 & nd & 101.01 \\
\hline ER-6 & ML & k1o & 3 & D-287100 & b & MDJ & 48.80 & 9.82 & 13.00 & nd & 17.30 & 8.10 & 1.63 & .27 & .00 & 1.73 & .18 & .18 & nd & 101.01 \\
\hline ER-7 & ML & K3 & 3 & D-287088 & $b$ & MDJ & 51.90 & 13.60 & 12.10 & nd & 7.04 & 10.80 & 2.22 & .42 & .00 & 2.47 & .27 & .17 & nd & 100.99 \\
\hline ER-8 & ML & k3 & 3 & D-313842 & b & JPL & 51.80 & 13.80 & 11.90 & nd & 6.85 & 10.80 & 2.28 & .43 & .19 & 2.46 & .27 & .16 & nd & 100.94 \\
\hline ER-9 & ML & K3 & 3 & D-313844 & b & JPL & 52.20 & 13.80 & 12.00 & nd & 6.55 & 10.90 & 2.38 & .44 & .00 & 2.51 & .28 & .17 & nd & 101.23 \\
\hline ER-10 & ML & $\mathrm{k} 3$ & 3 & D-313841 & b & JPL & 50.00 & 11.60 & 12.10 & nd & 12.60 & 9.24 & 1.94 & .33 & .00 & 2.09 & .22 & .16 & nd & 100.28 \\
\hline ER-11 & $\mathrm{ML}$ & k4 & 3 & D-313845 & $b$ & JPL & 51.80 & 13.80 & 12.00 & nd & 6.88 & 10.80 & 2.26 & .40 & .00 & 2.08 & .25 & .17 & nd & 100.44 \\
\hline ER-12 & ML & k2 & 3 & D-313843 & b & JPL & 48.90 & 9.98 & 13.00 & nd & 17.00 & 8.12 & 1.74 & .31 & .00 & 1.88 & .20 & .17 & nd & 101.30 \\
\hline ER-13 & $\mathrm{ML}$ & k5-1880-81 & 3 & D-313846 & $b$ & JPL & 52.00 & 13.40 & 12.30 & nd & 8.04 & 10.40 & 2.19 & .33 & .00 & 2.02 & .21 & .18 & nd & 101.07 \\
\hline ER-14 & ML & k3 & 3 & W-214657 & c & JPL & 52.30 & 13.30 & 4.30 & 7.00 & 8.60 & 10.20 & 2.30 & .48 & .53 & 2.00 & .32 & .12 & .00 & 101.45 \\
\hline ER-15 & ML & k4 & 3 & D-313847 & b & JPL & 51.90 & 13.90 & 12.10 & nd & 6.98 & 10.80 & 2.24 & .43 & .00 & 2.14 & .26 & .17 & nd & 100.92 \\
\hline ES-1 & KI & (p4y) & 2 & D-276918 & b & CAN & 50.90 & 14.10 & 12.00 & nd & 6.82 & 11.00 & 2.29 & .42 & .28 & 2.52 & .26 & .17 & nd & 100.76 \\
\hline $\mathrm{ES}-2$ & $\mathrm{KI}$ & p4o & 2 & D-284970 & b & CAN & 51.60 & 13.80 & 12.30 & nd & 6.92 & 11.20 & 2.30 & .41 & .00 & 2.47 & .26 & .17 & nd & 101.43 \\
\hline ES-4 & $\mathrm{K} \mathrm{I}$ & (p4o) & 2 & D-276920 & b & CAN & 51.40 & 13.90 & 12.10 & nd & 6.88 & 11.10 & 2.31 & .39 & .00 & 2.45 & .26 & .17 & nd & 100.96 \\
\hline ES-6 & $\mathrm{KI}$ & p5-1974 & 2 & D-284969 & b & CAN & 49.10 & 11.70 & 12.80 & nd & 12.90 & 9.76 & 1.97 & .40 & .00 & 2.26 & .24 & .18 & nd & 101.31 \\
\hline ES-7 & KI & p4o & 2 & D-276909 & b & CAN & 51.20 & 13.90 & 12.20 & nd & 7.12 & 11.00 & 2.35 & .42 & .00 & 2.48 & .26 & .17 & nd & 101.10 \\
\hline ES-8 & $\mathrm{KI}$ & p5-1790 & 2 & D-284968 & b & CAN & 49.80 & 11.80 & 13.40 & nd & 11.50 & 10.00 & 1.87 & .31 & .00 & 2.29 & .22 & .18 & nd & 101.37 \\
\hline ES-9 & $\mathrm{KI}$ & $p 4 y$ & 2 & D-276910 & b & CAN & 50.50 & 13.20 & 12.60 & nd & 8.03 & 11.30 & 2.02 & .39 & .00 & 2.49 & .24 & .17 & nd & 100.94 \\
\hline ES-10 & $\mathrm{KI}$ & p5-1971 & 2 & D-278815 & b & CAN & 50.40 & 13.40 & 12.60 & nd & 7.40 & 11.50 & 2.11 & .49 & .00 & 2.69 & .28 & .17 & nd & 101.04 \\
\hline ES-11 & $\mathrm{KI}$ & p4o & 2 & D-276911 & b & CAN & 50.70 & 13.70 & 12.20 & nd & 7.50 & 11.10 & 2.15 & .39 & .04 & 2.40 & .25 & .17 & nd & 100.60 \\
\hline
\end{tabular}


Table 1.-Chemical analyses-Continued

\begin{tabular}{|c|c|c|c|c|c|c|c|c|c|c|c|c|c|c|c|c|c|c|c|c|}
\hline $\begin{array}{l}\text { Sample } \\
\text { locality } \\
\text { No. }\end{array}$ & $\begin{array}{l}\text { Vol- } \\
\text { cano }\end{array}$ & Unit & $\begin{array}{c}\text { Map } \\
\text { sheet } \\
\text { No. }\end{array}$ & $\begin{array}{l}\text { Laboratory } \\
\text { No. }\end{array}$ & Remark & Collector & $\mathrm{SiO}_{2}$ & $\mathrm{Al}_{2} \mathrm{O}_{3}$ & $\mathrm{Fe}_{2} \mathrm{O}_{3}$ & $\mathrm{FeO}$ & $\mathrm{MgO}$ & $\mathrm{CaO}$ & $\mathrm{Na}_{2} \mathrm{O}$ & $\mathrm{K}_{2} \mathrm{O}$ & $\mathrm{H}_{2} \mathrm{O}$ & $\mathrm{TiO}_{2}$ & $\mathrm{P}_{2} \mathrm{O}_{5}$ & $\mathrm{MnO}$ & $\mathrm{CO}_{2}$ & Total \\
\hline ES-12 & $\mathrm{ML}$ & $\mathrm{k} 3$ & $\overline{2}$ & D-287087 & $b$ & MDJ & 48.90 & 10.10 & 12.60 & nd & 17.10 & 8.14 & 1.56 & .26 & .00 & 1.71 & .17 & .17 & nd & 100.71 \\
\hline ES-13 & KI & p5-1982 & 2 & D-278821 & b & CAN & 50.90 & 13.30 & 12.40 & nd & 7.73 & 11.50 & 2.14 & .46 & .00 & 2.61 & .27 & .17 & nd & 101.48 \\
\hline ES-14 & $\mathrm{KI}$ & p4o & 2 & D-276914 & b & CAN & 51.20 & 13.80 & 12.20 & nd & 7.12 & 11.00 & 2.13 & .39 & .31 & 2.42 & .25 & .17 & nd & 100.99 \\
\hline ES-15 & KI & (p4y) & 2 & D-276913 & b & CAN & 46.40 & 8.46 & 13.50 & nd & 22.10 & 6.95 & 1.40 & .28 & .00 & 1.61 & .18 & .17 & nd & 101.05 \\
\hline ES-16 & $\mathrm{K} \mathrm{I}$ & (p4o) & 2 & D-287580 & b & CAN & 51.40 & 13.80 & 12.20 & nd & 6.74 & 11.10 & 2.25 & .41 & .49 & 2.45 & .26 & .17 & nd & 101.27 \\
\hline ES-18 & $\mathrm{KI}$ & p5-1974 & 2 & D-278812 & b & CAN & 50.50 & 13.70 & 12.70 & nd & 7.12 & 11.20 & 2.20 & .46 & .00 & 2.61 & .26 & .17 & nd & 100.92 \\
\hline ES-19 & $\mathrm{KI}$ & p5-1974 & 2 & D-278813 & $b$ & CAN & 50.50 & 13.20 & 12.50 & nd & 7.19 & 11.40 & 2.10 & .47 & .05 & 2.59 & .26 & .17 & nd & 100.43 \\
\hline ES-20 & $\mathrm{KI}$ & p5-1919 & 2 & D-278822 & b & CAN & 49.50 & 12.40 & 12.70 & nd & 10.00 & 11.10 & 2.05 & .48 & .00 & 2.67 & .27 & .17 & nd & 101.34 \\
\hline ES-22 & $\mathrm{KI}$ & pa5-1790 & 2 & D-278827 & $b$ & CAN & 49.10 & 12.80 & 13.00 & nd & 10.20 & 9.93 & 1.89 & .44 & .44 & 2.38 & .27 & .17 & nd & 100.62 \\
\hline ES-25 & ML & $k 4$ & 2 & $\mathrm{NE}-45$ & a & JMR & 51.88 & 13.68 & 12.06 & nd & 6.31 & 10.37 & 2.57 & .47 & nd & 2.23 & .27 & .17 & nd & 100.01 \\
\hline ES-26 & KI & p5-1894 & 2 & D-278810 & b & CAN & 50.40 & 13.60 & 12.60 & nd & 7.35 & 11.40 & 2.22 & .51 & .00 & 2.76 & .29 & .17 & nd & 101.30 \\
\hline ES-27 & $\mathrm{KI}$ & p5-1889 & 2 & D-278816 & b & CAN & 50.40 & 13.40 & 12.30 & nd & 7.62 & 11.60 & 2.10 & .47 & .00 & 2.65 & .27 & .17 & nd & 100.98 \\
\hline ES-28 & KI & p5-1982 & 2 & D-278820 & b & CAN & 50.80 & 13.40 & 12.40 & nd & 7.36 & 11.70 & 2.11 & .46 & .00 & 2.60 & .26 & .17 & nd & 101.26 \\
\hline ES-29 & $\mathrm{KI}$ & $(p 40)$ & 2 & D-276912 & b & CAN & 51.00 & 13.80 & 12.20 & nd & 6.96 & 11.00 & 2.38 & .44 & .06 & 2.53 & .27 & .17 & nd & 100.81 \\
\hline ES-31 & $\mathrm{KI}$ & p4o & 2 & D-276916 & b & CAN & 51.20 & 13.90 & 12.10 & nd & 6.64 & 11.10 & 2.32 & .44 & .02 & 2.55 & .27 & .17 & nd & 100.71 \\
\hline ES-32 & $\mathrm{KI}$ & p5-1921 & 2 & D-278823 & $b$ & CAN & 50.20 & 13.50 & 12.50 & nd & 7.27 & 11.80 & 2.22 & .53 & .00 & 2.93 & .29 & .17 & nd & 101.41 \\
\hline ES-34 & KI & p5-1975 & 2 & D-278819 & b & CAN & 50.60 & 13.50 & 12.50 & nd & 7.35 & 11.60 & 2.18 & .47 & .00 & 2.61 & .26 & .17 & nd & 101.24 \\
\hline ES-35 & $\mathrm{KI}$ & p5-1959 & 2 & D-278828 & b & CAN & 48.00 & 10.90 & 13.00 & nd & 14.20 & 9.93 & 1.78 & .45 & .00 & 2.33 & .24 & .17 & nd & 101.00 \\
\hline ES-36 & $\mathrm{KI}$ & p5-1954 & 2 & D-278817 & $\mathrm{b}$ & CAN & 49.90 & 13.20 & 12.60 & nd & 7.34 & 11.80 & 2.13 & .54 & .00 & 2.84 & .03 & .17 & nd & 100.55 \\
\hline ES-38 & $\mathrm{KI}$ & p5-1974 & 2 & D-278811 & $\mathrm{b}$ & CAN & 50.00 & 12.90 & 12.70 & nd & 8.90 & 11.10 & 2.07 & .43 & .00 & 2.44 & .25 & .17 & nd & 100.96 \\
\hline ES-39 & $\mathrm{KI}$ & p5-1885 & 2 & D-278818 & $b$ & CAN & 50.40 & 14.10 & 12.10 & nd & 6.69 & 11.60 & 2.25 & .49 & .00 & 2.69 & .27 & .16 & nd & 100.75 \\
\hline ES-40 & KI & p5-1919 & 2 & D-278824 & b & CAN & 50.20 & 13.60 & 12.50 & nd & 7.00 & 11.80 & 2.25 & .53 & .00 & 2.91 & .29 & .17 & nd & 101.25 \\
\hline ES-41 & $\mathrm{ML}$ & k4 & 2 & D-276917 & b & CAN & 52.00 & 13.90 & 11.80 & nd & 6.84 & 10.80 & 2.38 & .42 & .00 & 2.16 & .26 & .16 & nd & 100.72 \\
\hline ES-42 & KI & p5-1971 & 2 & D-278814 & $\mathrm{b}$ & CAN & 50.30 & 13.20 & 12.70 & nd & 7.88 & 11.40 & 2.08 & .48 & .00 & 2.65 & .28 & .17 & nd & 101.14 \\
\hline ES-43 & $\mathrm{KI}$ & (p4o) & 2 & D-284965 & $\mathrm{b}$ & CAN & 50.90 & 13.10 & 12.60 & nd & 8.60 & 10.60 & 2.18 & .38 & .00 & 2.29 & .24 & .17 & nd & 101.06 \\
\hline ES-45 & $\mathrm{ML}$ & (k10) & 2 & D-313503 & $b$ & JPL & 51.30 & 13.80 & 12.20 & nd & 7.01 & 10.40 & 2.23 & .44 & .80 & 2.27 & .28 & .17 & nd & 100.90 \\
\hline ES-46 & $\mathrm{KI}$ & (p4o) & 2 & W-214684 & c & JPL & 50.00 & 14.40 & 2.80 & 8.50 & 7.50 & 10.40 & 2.00 & .40 & 1.50 & 2.10 & .30 & .19 & .10 & 100.19 \\
\hline ES-47 & $\mathrm{KI}$ & (p4o) & 2 & D-278829 & b & CAN & 50.70 & 13.30 & 12.60 & nd & 7.29 & 11.50 & 2.12 & .42 & .00 & 2.57 & .26 & .17 & nd & 100.93 \\
\hline ES-48 & $\mathrm{KI}$ & (p4o) & 2 & D-284963 & $b$ & CAN & 51.40 & 13.80 & 12.10 & nd & 7.27 & 11.10 & 2.30 & .39 & .00 & 2.35 & .25 & .17 & nd & 101.13 \\
\hline ES-49 & KI & (p4o) & 2 & D-284980 & b & CAN & 50.20 & 12.90 & 12.60 & nd & 8.15 & 11.20 & 2.15 & .40 & .14 & 2.48 & .25 & .17 & nd & 100.64 \\
\hline ES-50 & $\mathrm{KI}$ & (p4o) & 2 & D-284964 & b & CAN & 51.70 & 13.90 & 12.30 & nd & 6.89 & 11.30 & 2.29 & .37 & .00 & 2.45 & .25 & .17 & nd & 101.62 \\
\hline ES-51 & $\mathrm{ML}$ & $k 2$ & 2 & D-313502 & b & JPL & 51.90 & 13.60 & 12.50 & nd & 7.05 & 10.70 & 2.26 & .42 & .00 & 2.26 & .26 & .17 & nd & 101.12 \\
\hline ES-52 & $\mathrm{ML}$ & $\mathrm{k} 2$ & 2 & D-313504 & b & JPL & 49.90 & 12.60 & 13.40 & nd & 8.69 & 11.10 & 2.07 & .40 & .00 & 2.80 & .24 & .18 & nd & 101.38 \\
\hline ES-53 & ML & $k 3$ & 2 & NE-16 & a & JPL & 51.57 & 13.14 & 12.54 & nd & 6.95 & 10.07 & 2.44 & .53 & nd & 2.41 & .32 & .19 & nd & 100.16 \\
\hline ES-54 & ML & k4 & 2 & NE-15 & a & JPL & 52.20 & 13.83 & 11.91 & nd & 6.51 & 10.45 & 2.46 & .47 & nd & 2.23 & .27 & .17 & nd & 100.50 \\
\hline ES-55 & ML & $k 4$ & 2 & NE-17 & a & JPL & 51.91 & 13.67 & 11.56 & nd & 6.94 & 10.55 & 2.34 & .43 & nd & 2.04 & .25 & .17 & nd & 99.86 \\
\hline ES-57 & ML & k1y & 2 & NE-55 & a & JPL & 51.43 & 13.38 & 11.69 & nd & 7.72 & 10.28 & 2.50 & .44 & nd & 2.09 & .28 & .17 & nd & 99.98 \\
\hline ES-58 & ML & $\mathrm{k} 4$ & 2 & NE-18 & a & JPL & 52.28 & 13.91 & 11.70 & nd & 6.62 & 10.55 & 2.42 & .45 & nd & 2.12 & .27 & .18 & nd & 100.50 \\
\hline ET-1 & $\mathrm{KI}$ & $\mathrm{p} 40$ & 2 & D-284984 & $\mathrm{b}$ & CAN & 49.30 & 11.80 & 13.00 & nd & 12.40 & 10.10 & 1.99 & .39 & .00 & 2.23 & .23 & .18 & nd & 101.62 \\
\hline ET-2 & KI & p4y & 2 & D-284981 & $\mathrm{b}$ & CAN & 50.10 & 12.70 & 12.70 & nd & 9.06 & 10.80 & 2.08 & .42 & .00 & 2.53 & .26 & .18 & nd & 100.83 \\
\hline
\end{tabular}


Table 1.-Chemical analyses-Continued

\begin{tabular}{|c|c|c|c|c|c|c|c|c|c|c|c|c|c|c|c|c|c|c|c|c|}
\hline $\begin{array}{c}\text { Sample } \\
\text { locality } \\
\text { No. }\end{array}$ & $\begin{array}{l}\text { Vol- } \\
\text { cano }\end{array}$ & Unit & $\begin{array}{c}\text { Map } \\
\text { sheet } \\
\text { No. }\end{array}$ & $\begin{array}{l}\text { Laboratory } \\
\text { No. }\end{array}$ & Remark & Collector & $\mathrm{SiO}_{2}$ & $\mathrm{Al}_{2} \mathrm{O}_{3}$ & $\mathrm{Fe}_{2} \mathrm{O}_{3}$ & $\mathrm{FeO}$ & $\mathrm{MgO}$ & $\mathrm{CaO}$ & $\mathrm{Na}_{2} \mathrm{O}$ & $\mathrm{K}_{2} \mathrm{O}$ & $\mathrm{H}_{2} \mathrm{O}$ & $\mathrm{TiO}_{2}$ & $\mathrm{P}_{2} \mathrm{O}_{5}$ & $\mathrm{MnO}$ & $\mathrm{CO}_{2}$ & Total \\
\hline ET-3 & $\mathrm{K}$ & pc4y & 2 & D-284982 & $\mathrm{b}$ & CAN & 50.30 & 12.90 & 12.50 & nd & 9.26 & 10.90 & 2.06 & .39 & .00 & 2.41 & .24 & .18 & nd & 101.14 \\
\hline ET-4 & $\mathrm{K} \mathbf{I}$ & $\mathrm{p} 4$ & 2 & D-284971 & b & CAN & 51.00 & 13.20 & 12.30 & nd & 8.56 & 11.10 & 2.08 & .35 & .00 & 2.43 & .23 & .17 & nd & 101.42 \\
\hline ET-5 & $\mathrm{K} \mathbf{I}$ & pc3 & 2 & D-284983 & b & CAN & 49.10 & 11.50 & 12.90 & nd & 13.40 & 9.51 & 1.78 & .36 & .00 & 2.19 & .22 & .18 & nd & 101.14 \\
\hline ET-6 & $\mathrm{KI}$ & (p4) & 2 & D-284966 & b & CAN & 51.00 & 13.00 & 12.60 & nd & 8.09 & 11.30 & 2.15 & .38 & .00 & 2.57 & .24 & .18 & nd & 101.51 \\
\hline ET-7 & $\mathrm{K}$ & (p4o) & 2 & D-284978 & b & CAN & 51.00 & 13.50 & 12.40 & nd & 7.96 & 11.10 & 2.25 & .37 & .00 & 2.34 & .24 & .17 & nd & 101.33 \\
\hline ET-9 & $\mathrm{K}$ & $\mathrm{p} 4$ & 2 & D-287597 & b & CAN & 50.80 & 13.10 & 12.50 & nd & 8.34 & 11.00 & 2.09 & .39 & .00 & 2.51 & .24 & .18 & nd & 101.15 \\
\hline ET-10 & KI & p4 & 2 & D-287596 & b & CAN & 51.00 & 13.30 & 12.60 & nd & 7.26 & 11.50 & 2.07 & .40 & .00 & 2.57 & .24 & .18 & nd & 101.12 \\
\hline ET-11 & $\mathrm{KJ}$ & p4 & 2 & W-213215 & c & $\mathrm{RTH}$ & 51.00 & 13.10 & 6.60 & 5.40 & 7.60 & 11.00 & 2.10 & .44 & .08 & 2.40 & .29 & .17 & .01 & 100.19 \\
\hline ET-12 & $\mathrm{K}$ & (p2) & 2 & D-287595 & b & CAN & 50.80 & 13.50 & 12.90 & nd & 6.77 & 11.50 & 2.27 & .49 & .00 & 2.87 & .29 & .18 & nd & 101.57 \\
\hline ET-13 & $\mathrm{K}]$ & $\mathrm{p} 4$ & 2 & W-213234 & c & $\mathrm{RTH}$ & 50.00 & 12.30 & 1.30 & 10.30 & 10.50 & 9.90 & 2.00 & .44 & .06 & 2.30 & .27 & .19 & .01 & 99.57 \\
\hline ET-14 & ML & (k10?) & 2 & D-313501 & b & JPL & 51.30 & 13.90 & 12.20 & nd & 7.32 & 10.90 & 2.22 & .38 & .22 & 2.24 & .27 & .17 & nd & 101.12 \\
\hline ET-15 & $\mathrm{KI}$ & $\mathrm{p} 4$ & 2 & W-213216 & c & $\mathrm{RTH}$ & 51.40 & 13.60 & 2.00 & 9.40 & 7.10 & 10.70 & 2.10 & .46 & .17 & 2.50 & .30 & .18 & .01 & 99.92 \\
\hline EU-2 & $\mathrm{KI}$ & p2 & 2 & D-291780 & $b$ & RWH & 51.10 & 13.50 & 13.00 & nd & 6.43 & 10.40 & 2.43 & .52 & .00 & 2.97 & .31 & .18 & nd & 100.84 \\
\hline EU-3 & KI & p4y & 2 & D-291785 & $b$ & RWH & 50.70 & 13.70 & 13.00 & nd & 6.72 & 10.90 & 2.36 & .44 & .00 & 2.77 & .27 & .18 & nd & 101.04 \\
\hline EU-4 & KI & p4o & 2 & D-291781 & b & RWH & 50.60 & 13.50 & 12.80 & nd & 6.98 & 10.50 & 2.44 & .55 & .00 & 2.92 & .31 & .17 & nd & 100.77 \\
\hline EU-7 & KI & p4y & 2 & D-291779 & b & RWH & 50.60 & 13.20 & 13.60 & nd & 6.41 & 10.10 & 2.50 & .59 & .00 & 3.29 & .34 & .18 & nd & 100.81 \\
\hline EU-8 & KI & p4o & 2 & D-291777 & b & $\mathrm{RWH}$ & 50.80 & 13.40 & 13.70 & nd & 5.66 & 9.84 & 2.71 & .73 & .00 & 3.56 & .42 & .18 & nd & 101.00 \\
\hline EU-10 & $\mathrm{K}$ & p4 & 2 & D-291755 & b & RWH & 50.70 & 13.40 & 12.70 & nd & 7.45 & 11.10 & 2.23 & .49 & .00 & 2.78 & .28 & .17 & nd & 101.30 \\
\hline EU-11 & KI & $\mathrm{p} 4$ & 2 & D-291761 & b & RWH & 49.90 & 12.30 & 12.30 & nd & 10.50 & 10.50 & 1.97 & .38 & .00 & 2.41 & .23 & .17 & nd & 100.66 \\
\hline EU-12 & $\mathrm{KI}$ & p2 & 2 & D-291775 & b & RWH & 50.70 & 13.80 & 12.90 & nd & 6.64 & 10.90 & 2.30 & .44 & .00 & 2.74 & .26 & .18 & nd & 100.86 \\
\hline EU-13 & $\mathrm{KI}$ & p4y & 2 & D-291774 & b & RWH & 51.10 & 13.40 & 13.50 & nd & 6.16 & 9.92 & 2.56 & .62 & .00 & 3.20 & .36 & .18 & nd & 101.00 \\
\hline EU-14 & $\mathrm{K}]$ & p4y & 2 & D-291778 & b & RWH & 51.40 & 13.60 & 13.50 & nd & 5.56 & 9.64 & 2.73 & .71 & .00 & 3.50 & .42 & .18 & nd & 101.24 \\
\hline EU-15 & $\mathrm{KI}$ & p4 & 2 & D-291758 & b & $\mathrm{RWH}$ & 50.80 & 13.20 & 12.30 & nd & 7.84 & 11.50 & 2.14 & .40 & .00 & 2.50 & .24 & .17 & nd & 101.09 \\
\hline EU-16 & $\mathrm{K}$ & p4y & 2 & D-291776 & b & RWH & 51.30 & 13.50 & 13.40 & nd & 5.43 & 9.57 & 2.76 & .73 & .00 & 3.50 & .42 & .17 & nd & 100.78 \\
\hline EU-17 & Kl & p4y & 2 & D-291772 & b & RWH & 51.20 & 13.60 & 13.10 & nd & 5.91 & 10.10 & 2.60 & .63 & .00 & 3.27 & .37 & .17 & nd & 100.95 \\
\hline EU-18 & $\mathrm{KI}$ & $\mathrm{p} 4$ & 2 & D-291771 & b & $\mathrm{RWH}$ & 50.40 & 12.90 & 12.50 & nd & 8.71 & 10.90 & 2.10 & .41 & .20 & 2.57 & .25 & .17 & nd & 101.11 \\
\hline EU-19 & $\mathrm{KI}$ & p4 & 2 & D-291759 & b & RWH & 50.60 & 13.10 & 12.50 & nd & 7.91 & 11.40 & 2.03 & .40 & .25 & 2.53 & .24 & .17 & nd & 101.13 \\
\hline EU-20 & Kl & p4 & 2 & D-291757 & b & RWH & 49.20 & 11.40 & 12.50 & nd & 13.90 & 9.46 & 1.93 & .36 & .00 & 2.11 & .20 & .17 & nd & 101.23 \\
\hline EU-21 & $\mathrm{KI}$ & p4 & 2 & D-291756 & b & RWH & 50.10 & 12.90 & 12.30 & nd & 9.47 & 10.80 & 2.08 & .36 & .06 & 2.33 & .21 & .17 & nd & 100.78 \\
\hline EU-22 & $\mathrm{Kl}$ & p4o & 2 & D-291769 & b & RWH & 49.90 & 13.10 & 12.60 & nd & 8.15 & 10.70 & 2.29 & .59 & .04 & 2.95 & .32 & .17 & nd & 100.81 \\
\hline EU-23 & KI & p4 & 2 & W-213228 & c & $\mathrm{RTH}$ & 50.50 & 13.40 & 2.30 & 9.10 & 8.10 & 10.60 & 2.30 & .46 & .81 & 2.50 & .31 & .23 & .01 & 100.62 \\
\hline EU-24 & $\mathrm{KI}$ & $\mathrm{p} 4$ & 2 & D-291770 & b & RWH & 50.20 & 13.40 & 12.60 & nd & 7.20 & 10.80 & 2.39 & .62 & .04 & 3.08 & .34 & .17 & nd & 100.84 \\
\hline EU-25 & $\mathrm{KI}$ & p4o & 2 & D-291773 & b & RWH & 51.00 & 13.50 & 12.10 & nd & 7.23 & 11.40 & 2.17 & .43 & .00 & 2.67 & .27 & .17 & nd & 100.94 \\
\hline EU-26 & $\mathrm{Kl}$ & p4 & 2 & W-213227 & c & RTH & 48.90 & 11.00 & 2.10 & 9.80 & 14.50 & 9.00 & 1.80 & .40 & .24 & 2.00 & .24 & .20 & .01 & 100.19 \\
\hline EU-27 & KI & p4 & 2 & D-291760 & b & RWH & 49.30 & 11.70 & 12.40 & nd & 13.20 & 9.66 & 1.98 & .36 & .00 & 2.17 & .20 & .17 & nd & 101.14 \\
\hline EU-28 & $\mathrm{K}$ & p4 & 2 & D-291765 & b & RWH & 50.60 & 13.10 & 12.60 & nd & 8.26 & 11.20 & 2.13 & .41 & .00 & 2.56 & .25 & .17 & nd & 101.28 \\
\hline EU-29 & $\mathrm{Kl}$ & p4 & 2 & D-291764 & b & RWH & 50.40 & 13.20 & 12.70 & nd & 7.61 & 11.30 & 1.97 & .38 & .31 & 2.52 & .23 & .17 & nd & 100.79 \\
\hline EU-30 & $\mathrm{Kl}$ & p4 & 2 & D-291766 & b & $\mathrm{RWH}$ & 50.70 & 13.10 & 12.50 & nd & 7.88 & 11.30 & 2.14 & .40 & .00 & 2.56 & .25 & .17 & nd & 101.00 \\
\hline EU-31 & $\mathrm{KI}$ & p4 & 2 & D-291762 & b & RWH & 50.50 & 13.30 & 12.30 & nd & 7.54 & 11.50 & 2.11 & .39 & .00 & 2.47 & .24 & .17 & nd & 100.52 \\
\hline EU-32 & $\mathrm{K}$ & $\mathrm{p} 4$ & 2 & D-291767 & b & RWH & 50.70 & 13.20 & 12.70 & nd & 7.87 & 11.40 & 2.12 & .39 & .00 & 2.49 & .24 & .18 & nd & 101.29 \\
\hline
\end{tabular}


Table 1.-Chemical analyses-Continued

\begin{tabular}{|c|c|c|c|c|c|c|c|c|c|c|c|c|c|c|c|c|c|c|c|c|}
\hline $\begin{array}{c}\text { Sample } \\
\text { locality } \\
\text { No. }\end{array}$ & $\begin{array}{l}\text { Vol- } \\
\text { cano }\end{array}$ & Unit & $\begin{array}{c}\text { Map } \\
\text { sheet } \\
\text { No. }\end{array}$ & $\begin{array}{l}\text { Laboratory } \\
\text { No. }\end{array}$ & Remark & Collector & $\mathrm{SiO}_{2}$ & $\mathrm{Al}_{2} \mathrm{O}_{3}$ & $\mathrm{Fe}_{2} \mathrm{O}_{3}$ & $\mathrm{FeO}$ & $\mathrm{MgO}$ & $\mathrm{CaO}$ & $\mathrm{Na}_{2} \mathrm{O}$ & $\mathrm{K}_{2} \mathrm{O}$ & $\mathrm{H}_{2} \mathrm{O}$ & $\mathrm{TiO}_{2}$ & $\mathrm{P}_{2} \mathrm{O}_{5}$ & $\mathrm{MnO}$ & $\mathrm{CO}_{2}$ & Total \\
\hline EU-33 & $\mathrm{Kl}$ & p4 & 2 & D-291763 & $\bar{b}$ & RWH & 50.60 & 12.20 & 12.40 & nd & 11.90 & 9.57 & 1.97 & .35 & .00 & 1.88 & .22 & .17 & nd & 101.26 \\
\hline EU-34 & $\mathrm{KI}$ & p4 & 2 & D-291768 & b & RWH & 48.10 & 10.20 & 12.60 & nd & 17.40 & 8.50 & 1.75 & .28 & .00 & 1.80 & .17 & .17 & nd & 100.97 \\
\hline EV-1 & $\mathrm{KI}$ & p4y & 2 & W-214437 & c & RBM & 51.10 & 12.80 & 2.20 & 9.28 & 6.30 & 9.90 & 2.50 & .59 & .47 & 2.80 & .33 & .21 & .02 & 98.50 \\
\hline EV-2 & $\mathrm{KI}$ & p4y & 2 & W-214439 & c & RBM & 51.30 & 12.90 & 2.80 & 8.68 & 7.60 & 10.10 & 2.20 & .46 & .65 & 2.60 & .32 & .19 & .02 & 99.82 \\
\hline EV-3 & $\mathrm{KI}$ & p3 & 2 & W-214435 & c & RBM & 51.30 & 12.70 & 2.40 & 8.84 & 7.00 & 10.50 & 2.20 & .42 & .31 & 2.50 & .30 & .20 & .02 & 98.69 \\
\hline EV-4 & $\mathrm{KI}$ & p4y & 2 & W-214434 & c & RBM & 51.30 & 13.30 & 2.00 & 9.00 & 8.10 & 10.40 & 2.20 & .40 & .50 & 2.40 & .28 & .21 & .02 & 100.11 \\
\hline EV-5 & $\mathrm{KI}$ & p4o & 2 & W-214433 & c & RBM & 49.90 & 12.60 & 3.40 & 7.80 & 10.60 & 9.90 & 2.10 & .50 & .68 & 2.30 & .28 & .22 & .02 & 100.30 \\
\hline EV-6 & $\mathrm{KI}$ & p4y & 2 & W-214432 & c & RBM & 51.20 & 13.70 & 3.70 & 7.64 & 6.80 & 10.10 & 2.40 & .54 & .70 & 2.70 & .34 & .13 & .02 & 99.97 \\
\hline EV-7 & $\mathrm{KI}$ & $\mathrm{p} 4 \mathrm{y}$ & 2 & W-214446 & c & RBM & 51.30 & 13.10 & 1.50 & 9.68 & 6.90 & 10.30 & 2.30 & .44 & .36 & 2.60 & .29 & .19 & .02 & 98.98 \\
\hline EV-8 & $\mathrm{KI}$ & p5-1790 & 2 & W-214428 & c & RBM & 51.90 & 13.90 & 2.50 & 9.24 & 6.40 & 10.20 & 2.50 & .48 & .34 & 2.70 & .29 & .14 & .02 & 100.61 \\
\hline EV-9 & $\mathrm{K}$ & p3 & 2 & W-214427 & c & RBM & 50.00 & 12.90 & 1.50 & 10.00 & 8.60 & 9.90 & 2.20 & .49 & .51 & 2.50 & .32 & .13 & .02 & 99.07 \\
\hline EV-10 & $\mathrm{K}]$ & p4o & 2 & W-214426 & c & RBM & 51.10 & 13.50 & 1.60 & 10.20 & 6.40 & 9.80 & 2.60 & .60 & .41 & 3.00 & .36 & .16 & .02 & 99.75 \\
\hline EV-11 & $\mathrm{KI}$ & p4y & 2 & W-214445 & c & RBM & 51.50 & 13.30 & 1.80 & 9.56 & 6.70 & 10.40 & 2.50 & .44 & .33 & 2.60 & .29 & .18 & .02 & 99.62 \\
\hline EV-12 & $\mathrm{KI}$ & p4o & 2 & W-214425 & c & RBM & 51.10 & 13.80 & 1.80 & 10.40 & 6.30 & 10.00 & 2.40 & .56 & .47 & 3.00 & .34 & .16 & .02 & 100.35 \\
\hline EV-13 & KI & p4o & 2 & W-214424 & c & RBM & 51.20 & 13.10 & 1.70 & 10.12 & 6.40 & 9.90 & 2.40 & .56 & .33 & 3.00 & .39 & .15 & .02 & 99.27 \\
\hline EV-14 & KI & p3 & 2 & W-214423 & c & $\mathrm{RBM}$ & 50.20 & 11.60 & 2.10 & 9.00 & 13.00 & 8.70 & 2.10 & .38 & .55 & 2.10 & .26 & .14 & .02 & 100.15 \\
\hline EV-15 & $\mathrm{KI}$ & p4o & 2 & W-214422 & c & RBM & 51.90 & 13.00 & 2.80 & 9.92 & 5.00 & 7.80 & 3.00 & .96 & .67 & 3.50 & .59 & .17 & .02 & 99.33 \\
\hline EV-16 & $\mathrm{KI}$ & p5-1790 & 2 & W-214421 & c & RBM & 51.40 & 13.90 & 3.00 & 8.80 & 6.20 & 9.80 & 2.50 & .59 & .32 & 2.90 & .34 & .17 & .02 & 99.94 \\
\hline EV-17 & $\mathrm{KI}$ & p3 & 2 & W-214420 & c & RBM & 50.10 & 13.60 & 4.00 & 8.60 & 5.60 & 9.30 & 2.70 & .77 & .17 & 3.50 & .43 & .14 & .02 & 98.93 \\
\hline EV-18 & $\mathrm{KI}$ & p4o & 2 & W-214440 & c & RBM & 51.30 & 13.30 & 2.10 & 9.36 & 6.70 & 10.20 & 2.50 & .57 & .38 & 2.80 & .35 & .18 & .02 & 99.76 \\
\hline EV-19 & $\mathrm{KI}$ & p4y & 2 & W-214444 & c & RBM & 51.00 & 13.40 & 2.80 & 8.96 & 6.50 & 9.90 & 2.30 & .49 & .39 & 2.70 & .30 & .19 & .02 & 98.95 \\
\hline EV-20 & $\mathrm{KI}$ & p4y & 2 & W-214441 & c & RBM & 51.80 & 13.00 & 1.60 & 9.72 & 7.10 & 10.40 & 2.30 & .46 & .33 & 2.50 & .30 & .21 & .02 & 99.74 \\
\hline EV-22 & $\mathrm{KI}$ & p4y & 2 & W-214442 & c & RBM & 51.60 & 13.20 & 1.60 & 9.72 & 7.20 & 10.30 & 2.30 & .42 & .41 & 2.50 & .31 & .20 & 02 & 99.78 \\
\hline EV-23 & $\mathrm{KI}$ & p4y & 2 & W-214457 & c & RBM & 51.50 & 13.40 & 1.40 & 9.70 & 6.70 & 10.50 & 2.30 & .47 & .38 & 2.60 & .31 & .16 & .02 & 99.44 \\
\hline EV-24 & $\mathrm{KI}$ & p4o & 2 & W-212925 & c & RBM & 51.50 & 13.70 & 3.70 & 8.20 & 6.00 & 9.60 & 1.90 & .59 & .74 & 2.90 & .41 & .18 & .01 & 99.43 \\
\hline EV-25 & $\mathrm{K}$ & p4o & 2 & W-214419 & c & RBM & 51.80 & 13.50 & 3.10 & 10.80 & 4.90 & 8.90 & 2.80 & .80 & .39 & 3.70 & .46 & .15 & .02 & 101.32 \\
\hline EV-26 & $\mathrm{K}$ & p3 & 2 & W-216966 & c & RBM & 51.00 & 13.60 & 1.80 & 9.50 & 8.20 & 10.30 & 2.40 & .44 & .64 & 2.50 & .28 & .13 & .01 & 100.80 \\
\hline EV-27 & $\mathrm{KI}$ & p3 & 2 & W-214410 & c & RBM & 51.00 & 13.80 & 4.10 & 7.92 & 6.60 & 10.20 & 2.50 & .48 & .42 & 2.70 & .32 & .16 & .02 & 100.22 \\
\hline EV-28 & KI & p4y & 2 & W-214456 & c & RBM & 53.70 & 13.00 & 2.10 & 8.50 & 5.90 & 8.90 & 2.10 & .59 & 1.55 & 3.00 & .35 & .14 & .02 & 99.85 \\
\hline EV-29 & $\mathrm{KI}$ & p4y & 2 & W-214414 & c & RBM & 50.70 & 13.80 & 6.60 & 5.28 & 6.60 & 10.30 & 2.50 & .43 & .47 & 2.60 & .31 & .14 & .02 & 99.75 \\
\hline EV-30 & $\mathrm{KI}$ & p4o & 2 & W-214450 & c & RBM & 51.30 & 13.20 & 3.30 & 8.40 & 6.20 & 9.90 & 2.40 & .57 & .32 & 2.80 & .33 & .18 & .02 & 98.92 \\
\hline EV-31 & $\mathrm{KI}$ & p4o & 2 & W-214455 & c & RBM & 51.00 & 13.20 & 2.20 & 9.60 & 6.90 & 9.70 & 2.50 & .70 & .49 & 2.90 & .34 & .14 & .02 & 99.69 \\
\hline EV-32 & $\mathrm{KI}$ & p4y & 2 & W-212931 & c & RBM & 50.00 & 13.50 & 1.50 & 10.20 & 8.80 & 9.80 & 2.20 & .41 & .52 & 2.60 & .32 & .18 & .01 & 100.04 \\
\hline EV-33 & $\mathrm{K}$ & p4o & 2 & W-214451 & c & RBM & 51.40 & 13.40 & 3.60 & 8.00 & 6.00 & 9.40 & 2.60 & .73 & .52 & 3.00 & .43 & .18 & .02 & 99.28 \\
\hline EV-34 & $\mathrm{KI}$ & pc3 & 2 & W-214409 & c & RBM & 50.60 & 13.40 & 7.70 & 4.80 & 7.90 & 9.60 & 2.20 & .43 & .68 & 2.60 & .32 & .16 & .02 & 100.41 \\
\hline EV-35 & $\mathrm{KI}$ & p3 & 2 & W-210993 & c & RBM & 50.10 & 14.50 & 4.00 & 9.10 & 5.50 & 9.10 & 2.40 & .55 & 1.33 & 3.70 & .55 & .17 & .02 & 101.02 \\
\hline EV-36 & KI & pc3 & 2 & W-210994 & c & RBM & 49.00 & 11.50 & 1.40 & 10.60 & 13.90 & 9.00 & 1.70 & .22 & .39 & 1.90 & .21 & .11 & .02 & 99.95 \\
\hline EV-37 & $\mathrm{KI}$ & p4o & 2 & W-210992 & c & RBM & 49.70 & 13.20 & 3.20 & 8.90 & 9.40 & 9.70 & 2.20 & .39 & .53 & 2.50 & .33 & .15 & .01 & 100.21 \\
\hline EV-38 & $\mathrm{KI}$ & p3 & 2 & W-212930 & c & RBM & 50.10 & 13.40 & 7.00 & 5.80 & 6.10 & 9.70 & 2.20 & .52 & .94 & 3.10 & .39 & .19 & .01 & 99.45 \\
\hline EV-39 & $\mathrm{KI}$ & p4o & 2 & W-214454 & c & RBM & 51.70 & 13.20 & 1.30 & 10.40 & 5.90 & 9.40 & 2.50 & .64 & .61 & 3.10 & .37 & .17 & .02 & 99.31 \\
\hline
\end{tabular}


Table 1.-Chemical analyses-Continued

\begin{tabular}{|c|c|c|c|c|c|c|c|c|c|c|c|c|c|c|c|c|c|c|c|c|}
\hline $\begin{array}{c}\text { Sample } \\
\text { locality } \\
\text { No. }\end{array}$ & $\begin{array}{l}\text { Vol- } \\
\text { cano }\end{array}$ & Unit & $\begin{array}{c}\text { Map } \\
\text { sheet } \\
\text { No. }\end{array}$ & $\begin{array}{l}\text { Laboratory } \\
\text { No. }\end{array}$ & Remark & Collector & $\mathrm{SiO}_{2}$ & $\mathrm{Al}_{2} \mathrm{O}_{3}$ & $\mathrm{Fe}_{2} \mathrm{O}_{3}$ & $\mathrm{FeO}$ & $\mathrm{MgO}$ & $\mathrm{CaO}$ & $\mathrm{Na}_{2} \mathrm{O}$ & $\mathrm{K}_{2} \mathrm{O}$ & $\mathrm{H}_{2} \mathrm{O}$ & $\mathrm{TiO}_{2}$ & $\mathrm{P}_{2} \mathrm{O}_{5}$ & $\mathrm{MnO}$ & $\mathrm{CO}_{2}$ & Total \\
\hline EV-40 & $\mathrm{KI}$ & $p 3$ & 2 & W-214452 & $\mathrm{c}$ & RBM & 47.70 & 9.10 & 1.20 & 10.80 & 18.70 & 7.00 & 1.60 & .41 & .44 & 1.90 & .24 & .17 & .02 & 99.28 \\
\hline EV-41 & $\mathrm{KI}$ & p3 & 2 & W-212926 & c & RBM & 51.80 & 13.80 & 11.10 & 1.80 & 5.80 & 9.50 & 1.80 & .34 & .20 & 3.00 & .39 & .19 & .01 & 99.73 \\
\hline EV-43 & $\mathrm{KI}$ & p4o & 2 & W-214453 & c & RBM & 51.20 & 13.10 & 2.90 & 9.40 & 5.50 & 9.10 & 2.60 & .74 & .49 & 3.40 & .45 & .16 & .02 & 99.06 \\
\hline EV-44 & $\mathrm{K}]$ & p3 & 2 & W-216957 & c & RBM & 50.50 & 14.20 & 4.20 & 7.10 & 6.80 & 10.40 & 2.30 & .46 & .69 & 2.70 & .31 & .18 & .01 & 99.85 \\
\hline EV-45 & $\mathrm{KI}$ & p5-1790 & 2 & W-210954 & c & RBM & 50.00 & 14.20 & 2.20 & 9.00 & 7.30 & 10.00 & 2.30 & .26 & .42 & 2.40 & .30 & .14 & .02 & 98.54 \\
\hline EV-46 & $\mathrm{KI}$ & pc3 & 2 & W-216969 & c & RBM & 48.80 & 14.10 & 11.70 & .92 & 6.20 & 9.20 & 2.50 & .57 & 1.83 & 3.00 & .38 & .14 & .02 & 99.36 \\
\hline EV-47 & $\mathrm{Kl}$ & pc3 & 2 & W-210990 & c & RBM & 50.40 & 13.90 & 2.40 & 10.30 & 5.90 & 9.60 & 2.60 & .51 & .46 & 3.20 & .41 & .21 & .02 & 99.91 \\
\hline EV-48 & $\mathrm{KI}$ & p3 & 2 & W-214458 & c & RBM & 51.20 & 13.40 & 6.60 & 6.00 & 6.20 & 9.40 & 2.10 & .58 & .64 & 3.00 & .34 & .15 & .02 & 99.63 \\
\hline EV-49 & $\mathrm{KI}$ & p4o & 2 & W-216985 & c & RBM & 50.90 & 14.40 & 1.30 & 10.00 & 6.50 & 10.10 & 2.40 & .44 & .54 & 2.80 & .32 & .17 & .01 & 99.88 \\
\hline EV-50 & $\mathrm{KI}$ & pc3 & 2 & W-216970 & $c$ & RBM & 51.30 & 13.70 & 3.20 & 10.20 & 5.00 & 8.70 & 2.90 & .81 & .66 & 3.70 & .45 & .17 & .01 & 100.80 \\
\hline EV-51 & $\mathrm{KI}$ & p4o & 2 & W-216983 & c & RBM & 51.60 & 14.00 & 6.80 & 6.00 & 5.30 & 9.00 & 2.70 & .65 & .55 & 3.50 & .41 & .22 & .01 & 100.74 \\
\hline EV-52 & KI & p4o & 2 & W-210989 & c & RBM & 50.20 & 14.10 & 2.70 & 8.60 & 7.10 & 10.00 & 2.20 & .37 & .51 & 2.70 & .33 & .18 & .02 & 99.01 \\
\hline EV-53 & KI & pc4o & 2 & W-210947 & c & RBM & 50.90 & 13.70 & 4.70 & 8.20 & 5.50 & 8.90 & 2.50 & .48 & .26 & 3.40 & .45 & .06 & .02 & 99.07 \\
\hline EV-55 & $\mathrm{KI}$ & p5-1790 & 2 & W-210982 & c & RBM & 50.10 & 13.80 & 3.30 & 7.20 & 8.80 & 10.80 & 2.20 & .35 & .27 & 2.10 & .25 & .14 & .01 & 99.32 \\
\hline EV-56 & $\mathrm{KI}$ & p4o & 2 & W-210986 & c & RBM & 50.80 & 13.70 & 2.20 & 10.20 & 5.40 & 8.90 & 2.80 & .64 & .37 & 3.50 & .49 & .21 & .02 & 99.23 \\
\hline EV-57 & $\mathrm{KI}$ & p5-1840 & 2 & W-216979 & c & RBM & 51.00 & 14.20 & 1.60 & 9.80 & 6.90 & 10.40 & 2.40 & .45 & .56 & 2.70 & .30 & .15 & .01 & 100.47 \\
\hline EV-58 & $\mathrm{KI}$ & p4o & 2 & W-210949 & c & RBM & 50.20 & 13.90 & 8.00 & 4.70 & 6.00 & 9.60 & 2.40 & .39 & .64 & 3.10 & .40 & .07 & .02 & 99.42 \\
\hline EV-59 & $\mathrm{K} \mathbf{I}$ & p4o & 2 & W-216976 & c & RBM & 49.10 & 13.00 & 2.80 & 8.50 & 10.20 & 9.70 & 2.00 & .37 & 1.06 & 2.30 & .26 & .16 & .02 & 99.47 \\
\hline EV-60 & $\mathrm{KI}$ & p4o & 2 & W-210946 & c & RBM & 51.70 & 13.70 & 1.90 & 9.10 & 6.60 & 9.10 & 2.30 & .35 & .46 & 2.80 & .34 & .13 & .02 & 98.50 \\
\hline EV-62 & $\mathrm{Kl}$ & p4o & 2 & W-216977 & c & RBM & 50.70 & 14.20 & 4.00 & 8.70 & 5.50 & 9.00 & 2.60 & .62 & 1.27 & 3.80 & .42 & .17 & .01 & 100.99 \\
\hline EV-63 & $\mathrm{KI}$ & p4y & 2 & W-210981 & c & RBM & 51.30 & 13.90 & 1.80 & 9.40 & 7.20 & 10.20 & 2.30 & .43 & .37 & 2.60 & .31 & .17 & .02 & 100.00 \\
\hline EV-65 & $\mathrm{KI}$ & p4o & 2 & W-216958 & c & RBM & 51.40 & 14.20 & 2.20 & 9.90 & 5.70 & 9.30 & 2.70 & .63 & .52 & 3.30 & .39 & .19 & .02 & 100.45 \\
\hline EV-67 & $\mathrm{KI}$ & p4o & 2 & W-210987 & c & RBM & 50.70 & 14.10 & 2.00 & 9.40 & 6.40 & 9.60 & 2.40 & .48 & .26 & 2.80 & .35 & .14 & .02 & 98.65 \\
\hline EV-68 & $\mathrm{KI}$ & p4o & 2 & W-210988 & c & RBM & 50.40 & 14.30 & 6.70 & 5.40 & 6.70 & 9.60 & 2.40 & .45 & .39 & 2.70 & .33 & .23 & .02 & 99.62 \\
\hline EV-69 & $\mathrm{KI}$ & p4o & 2 & W-216972 & c & RBM & 51.40 & 14.30 & 1.90 & 10.20 & 5.90 & 9.70 & 2.70 & .63 & .56 & 3.30 & .36 & .18 & .02 & 101.15 \\
\hline EV-70 & KI & p5-1790 & 2 & W-210977 & c & RBM & 50.10 & 13.20 & 3.30 & 7.80 & 8.80 & 11.10 & 1.90 & .38 & .36 & 2.20 & .27 & .06 & .02 & 99.49 \\
\hline EV-71 & KI & $\mathrm{p} 40$ & 2 & W-210952 & c & RBM & 51.50 & 14.00 & 8.10 & 5.10 & 6.20 & 10.20 & 2.30 & .28 & .35 & 2.80 & .34 & .11 & .02 & 101.30 \\
\hline EV-72 & $\mathrm{KI}$ & p4y & 2 & W-212914 & c & RBM & 50.40 & 13.30 & 2.40 & 9.30 & 7.70 & 10.20 & 2.00 & .45 & .47 & 2.60 & .33 & .12 & .01 & 99.28 \\
\hline EV-73 & $\mathrm{K}]$ & p4o & 2 & W-216973 & c & RBM & 49.30 & 13.30 & 4.80 & 7.00 & 8.60 & 9.90 & 2.00 & .39 & 1.20 & 2.50 & .29 & .18 & .02 & 99.48 \\
\hline EV-74 & $\mathrm{KI}$ & p4o & 2 & W-216978 & c & RBM & 50.30 & 13.40 & 2.20 & 9.60 & 8.50 & 10.40 & 2.10 & .38 & .84 & 2.40 & .26 & .16 & .01 & 100.55 \\
\hline EV-77 & KI & pc3 & 2 & W-210979 & c & RBM & 50.40 & 13.70 & 1.80 & 10.90 & 5.10 & 8.50 & 3.00 & .86 & .72 & 3.80 & .55 & .12 & .02 & 99.47 \\
\hline EV-78 & $\mathrm{KI}$ & p4o & 2 & W-216971 & c & RBM & 50.10 & 13.00 & 2.00 & 9.80 & 10.10 & 9.90 & 2.10 & .35 & .54 & 2.30 & .25 & .15 & .03 & 100.62 \\
\hline EV-79 & $\mathrm{KI}$ & p5-1790 & 2 & W-210971 & c & RBM & 50.00 & 14.20 & 6.60 & 4.80 & 8.50 & 11.20 & 2.10 & .25 & .38 & 2.20 & .25 & .14 & .02 & 100.64 \\
\hline EV-80 & $\mathrm{K} I$ & pc3 & 2 & W-210973 & c & RBM & 50.10 & 13.80 & 3.90 & 8.60 & 8.20 & 10.30 & 2.00 & .34 & .46 & 2.70 & .34 & .08 & .01 & 100.83 \\
\hline EV-81 & KI & pc3 & 2 & W-210941 & c & RBM & 51.30 & 13.80 & 2.90 & 9.60 & 7.30 & 10.20 & 2.30 & .32 & .32 & 2.70 & .34 & .11 & .02 & 101.21 \\
\hline EV-82 & $\mathrm{KI}$ & pc4y & 2 & W-210943 & c & RBM & 50.00 & 13.90 & 4.10 & 8.20 & 6.50 & 10.70 & 2.30 & .31 & .71 & 2.80 & .36 & .10 & .03 & 100.01 \\
\hline EV-83 & $\mathrm{Kl}$ & p5-1840 & 2 & W-216955 & c & RBM & 50.30 & 14.10 & 3.40 & 9.00 & 5.60 & 9.00 & 2.50 & .62 & 1.43 & 3.40 & .38 & .17 & .02 & 99.92 \\
\hline EV-84 & $\mathrm{K}]$ & pc3 & 2 & W-210969 & c & RBM & 51.20 & 14.40 & 3.00 & 9.20 & 6.60 & 10.60 & 2.50 & .39 & .24 & 2.80 & .35 & .19 & .01 & 101.48 \\
\hline EV-85 & $\mathrm{KI}$ & p4y & 2 & W-210976 & c & RBM & 50.10 & 13.70 & 8.70 & 2.40 & 8.50 & 10.80 & 2.20 & .37 & .27 & 2.10 & .24 & .11 & .01 & 99.50 \\
\hline EV-86 & KI & pc3 & 2 & W-210972 & c & RBM & 50.40 & 14.20 & 5.40 & 8.40 & 5.50 & 9.40 & 2.50 & .46 & .76 & 3.30 & .43 & .18 & .02 & 100.95 \\
\hline
\end{tabular}


Table 1.-Chemical analyses-Continued

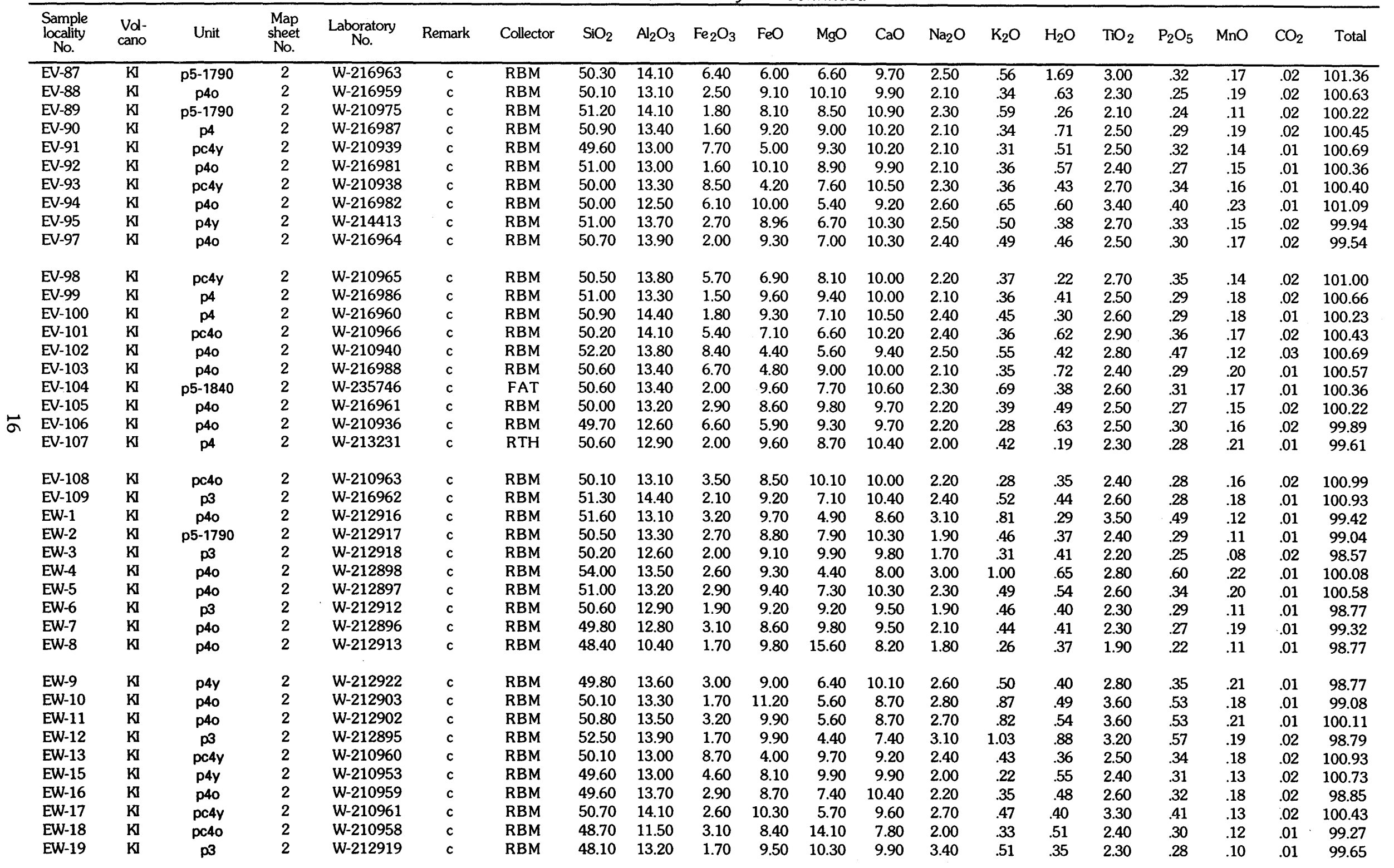


Table 1.-Chemical analyses-Continued

\begin{tabular}{|c|c|c|c|c|c|c|c|c|c|c|c|c|c|c|c|c|c|c|c|c|}
\hline $\begin{array}{c}\text { Sample } \\
\text { locality } \\
\text { No. }\end{array}$ & $\begin{array}{l}\text { Vol- } \\
\text { cano }\end{array}$ & Unit & $\begin{array}{l}\text { Map } \\
\text { sheet } \\
\text { No. }\end{array}$ & $\begin{array}{c}\text { Laboratory } \\
\text { No. }\end{array}$ & Remark & Collector & $\mathrm{SiO}_{2}$ & $\mathrm{Al}_{2} \mathrm{O}_{3}$ & $\mathrm{Fe}_{2} \mathrm{O}_{3}$ & $\mathrm{FeO}$ & $\mathrm{MgO}$ & $\mathrm{CaO}$ & $\mathrm{Na}_{2} \mathrm{O}$ & $\mathrm{K}_{2} \mathrm{O}$ & $\mathrm{H}_{2} \mathrm{O}$ & $\mathrm{TO}_{2}$ & $\mathrm{P}_{2} \mathrm{O}_{5}$ & $\mathrm{MnO}$ & $\mathrm{CO}_{2}$ & Total \\
\hline EW-20 & $\mathrm{KI}$ & $\mathrm{p} 4 \mathrm{y}$ & 2 & W-210957 & c & $\mathrm{RBM}$ & 51.20 & 14.00 & 2.50 & 10.30 & 6.00 & 10.00 & 2.60 & .46 & .49 & 3.00 & .39 & .12 & .02 & 101.08 \\
\hline EW-22 & $\mathrm{KI}$ & p4y & 2 & W-210968 & c & RBM & 50.00 & 13.50 & 5.10 & 6.40 & 9.30 & 10.20 & 2.10 & .29 & .40 & 2.30 & .29 & .14 & .02 & 100.04 \\
\hline EW-23 & $\mathrm{Kl}$ & p4o & 2 & W-212920 & c & RBM & 50.20 & 13.20 & 3.20 & 8.00 & 8.70 & 10.00 & 1.90 & .34 & .32 & 2.40 & .29 & .10 & .01 & 98.66 \\
\hline EW-24 & $\mathrm{KI}$ & p4y & 2 & W-210962 & c & RBM & 50.60 & 13.70 & 3.00 & 9.60 & 8.10 & 10.80 & 2.20 & .32 & .20 & 2.60 & .31 & .19 & .02 & 101.64 \\
\hline EW-25 & $\mathrm{K}]$ & pc4y & 2 & W-210956 & c & RBM & 50.50 & 13.70 & 2.30 & 9.00 & 8.30 & 9.70 & 2.30 & .32 & .71 & 2.50 & .29 & .08 & .02 & 99.72 \\
\hline EW-26 & $\mathrm{KI}$ & p5-1790 & 2 & W-212904 & c & RBM & 52.90 & 14.00 & 1.90 & 8.90 & 6.90 & 10.10 & 2.00 & .47 & .32 & 2.50 & .32 & .16 & .01 & 100.48 \\
\hline EW-27 & KI & $\mathrm{p} 40$ & 2 & W-212907 & c & RBM & 51.00 & 13.20 & 2.30 & 9.40 & 8.70 & 10.50 & 2.40 & .30 & .35 & 2.50 & .31 & .14 & .02 & 101.12 \\
\hline EW-28 & $\mathrm{KI}$ & p4o & 2 & W-212921 & c & RBM & 50.10 & 11.40 & 2.30 & 9.40 & 11.20 & 8.30 & 1.90 & .45 & .50 & 2.50 & .35 & .18 & .01 & 98.59 \\
\hline EW-29 & $\mathrm{Kl}$ & p5-1790 & 2 & W-212905 & c & RBM & 51.90 & 13.90 & 2.20 & 9.10 & 6.80 & 10.40 & 1.90 & .30 & .29 & 2.40 & .27 & .14 & .02 & 99.62 \\
\hline EW-30 & $\mathrm{KI}$ & pc4o & 2 & W-210955 & c & RBM & 50.10 & 13.70 & 3.30 & 8.20 & 7.30 & 9.30 & 2.50 & .51 & .45 & 3.00 & .38 & .17 & .02 & 98.93 \\
\hline EW-32 & $\mathrm{KI}$ & $p 40$ & 2 & W-210974 & c & RBM & 49.30 & 13.10 & 4.20 & 7.80 & 10.40 & 10.00 & 2.00 & .28 & .75 & 2.40 & .30 & .08 & .01 & 100.62 \\
\hline EW-33 & $\mathrm{K}]$ & p5-1790 & 2 & W-212909 & c & RBM & 50.70 & 13.90 & 1.50 & 9.70 & 6.60 & 9.70 & 2.70 & .41 & .25 & 2.70 & .37 & .13 & .01 & 98.67 \\
\hline EW-34 & $\mathrm{KI}$ & p5-1790 & 2 & W-212910 & c & RBM & 50.40 & 12.40 & 2.50 & 8.80 & 11.50 & 9.60 & 2.20 & .38 & .25 & 2.10 & .26 & .13 & .01 & 100.53 \\
\hline EW-35 & $\mathrm{KI}$ & p3 & 2 & W-212908 & c & RBM & 50.00 & 11.30 & 2.00 & 9.40 & 14.60 & 8.80 & 2.60 & .21 & .18 & 1.90 & .25 & .14 & .01 & 101.39 \\
\hline EW-36 & $\mathrm{KI}$ & p5-1790 & 2 & W-212899 & c & RBM & 50.80 & 13.00 & 2.40 & 9.50 & 8.50 & 9.70 & 2.30 & .49 & .41 & 2.50 & .32 & .19 & .01 & 100.12 \\
\hline EW-37 & $\mathrm{K}]$ & p3 & 2 & W-212911 & c & RBM & 50.50 & 13.70 & 4.10 & 7.60 & 7.40 & 9.50 & 2.00 & .37 & .49 & 2.50 & .30 & .11 & .01 & 98.58 \\
\hline EW-38 & $\mathrm{KI}$ & p4y & 2 & W-212900 & c & RBM & 50.40 & 13.60 & 2.60 & 9.40 & 7.10 & 10.50 & 1.90 & .47 & .39 & 2.70 & .33 & .22 & .01 & 99.62 \\
\hline EW-39 & $\mathrm{Kl}$ & p3 & 2 & W-212924 & c & RBM & 50.10 & 11.60 & 1.80 & 9.40 & 12.80 & 8.20 & 1.50 & .30 & .40 & 2.00 & .28 & .18 & .01 & 98.57 \\
\hline EW-40 & $\mathrm{KI}$ & p5-1840 & 2 & W-212901 & c & RBM & 47.70 & 9.70 & 2.10 & 9.70 & 18.00 & 7.90 & 1.70 & .28 & .30 & 1.80 & .21 & .16 & .01 & 99.56 \\
\hline FN-1 & $\mathrm{ML}$ & k & 1 & W-214689 & c & RBM & 51.70 & 13.20 & 2.10 & 8.64 & 7.20 & 10.00 & 2.30 & .37 & .53 & 2.10 & .28 & .19 & .02 & 98.63 \\
\hline FN-2 & ML & $k$ & 1 & W-214690 & c & RBM & 47.30 & 7.70 & 1.60 & 10.00 & 23.80 & 6.40 & 1.40 & .27 & .31 & 1.20 & .17 & .17 & .02 & 100.34 \\
\hline FN-3 & HU & $\mathrm{h}$ & 1 & W-214686 & c & RBM & 47.00 & 10.90 & 1.80 & 10.36 & 14.70 & 9.30 & 2.10 & .56 & .24 & 1.90 & .20 & .18 & .02 & 99.26 \\
\hline FO-1 & ML & $\mathrm{k} 3$ & 1 & D-313513 & b & JPL & 49.00 & 9.63 & 12.70 & nd & 18.00 & 7.95 & 1.55 & .24 & .00 & 1.61 & .16 & .17 & nd & 101.01 \\
\hline FO-2 & ML & k4 & 1 & D-313514 & $b$ & JPL & 51.90 & 13.80 & 12.00 & nd & 7.14 & 10.80 & 2.19 & .44 & .00 & 2.18 & .27 & .16 & nd & 100.88 \\
\hline FO-3 & ML & $k 2$ & 1 & D-313515 & b & JPL & 51.80 & 13.60 & 12.50 & nd & 7.32 & 10.60 & 2.20 & .32 & .00 & 2.20 & .23 & .17 & nd & 100.94 \\
\hline FO-4 & ML & $\mathrm{k} 2$ & 1 & D-313516 & b & JPL & 49.60 & 10.90 & 13.30 & nd & 14.40 & 8.91 & 1.77 & .27 & .00 & 1.85 & .19 & .18 & nd & 101.37 \\
\hline FO-5 & ML & $k 10$ & 1 & W-227935 & c & $\mathrm{RBM}+\mathrm{DAC}$ & 51.30 & 13.80 & 3.30 & 8.20 & 7.10 & 10.30 & 2.10 & .40 & .75 & 2.10 & .34 & .17 & .01 & 99.87 \\
\hline FO-6 & ML & $k 3$ & 1 & D-313517 & b & JPL & 52.30 & 13.90 & 12.00 & nd & 6.82 & 10.70 & 2.30 & .45 & .00 & 2.21 & .27 & .17 & nd & 101.12 \\
\hline FO-7 & $\mathrm{HU}$ & h1o & 1 & W-227939 & c & $\mathrm{RBM}+\mathrm{DAC}$ & 47.00 & 14.10 & 8.20 & 4.50 & 9.80 & 11.70 & 1.90 & .46 & .34 & 1.70 & .26 & .18 & .01 & 100.15 \\
\hline FO-8 & $\mathrm{HU}$ & hc1o & 1 & W-227941 & c & $\mathrm{RBM}+\mathrm{DAC}$ & 47.50 & 14.00 & 6.30 & 6.10 & 10.50 & 10.80 & 2.20 & .48 & .35 & 1.70 & .28 & .18 & .04 & 100.43 \\
\hline FO-9 & $\mathrm{HU}$ & hc1o & 1 & W-227940 & c & $\mathrm{RBM}+\mathrm{DAC}$ & 47.70 & 14.20 & 4.80 & 7.80 & 9.00 & 11.00 & 2.40 & .72 & .27 & 1.90 & .35 & .19 & .06 & 100.39 \\
\hline FO-10 & $\mathrm{HU}$ & h1o & 1 & W-227942 & c & $\mathrm{RBM}+\mathrm{DAC}$ & 47.70 & 14.20 & 3.80 & 8.40 & 9.40 & 11.40 & 2.30 & .10 & .40 & 1.70 & .23 & .18 & .00 & 99.81 \\
\hline FO-12 & HU & h10 & 1 & W-227937 & c & $\mathrm{RBM}+\mathrm{DAC}$ & 47.60 & 14.20 & 3.60 & 8.60 & 9.20 & 11.30 & 2.20 & .70 & .29 & 1.80 & .32 & .18 & .01 & 100.00 \\
\hline FO-13 & $\mathrm{HU}$ & hio & 1 & W-227945 & c & $\mathrm{RBM}+\mathrm{DAC}$ & 48.40 & 14.40 & 4.70 & 7.80 & 9.60 & 11.10 & 2.10 & .58 & .38 & 1.80 & .30 & .18 & .02 & 101.36 \\
\hline FO-14 & HU & h1o & 1 & W-227944 & c & $\mathrm{RBM}+\mathrm{DAC}$ & 48.50 & 14.50 & 3.20 & 9.20 & 8.90 & 11.60 & 2.30 & .61 & .10 & 1.90 & .31 & .18 & .00 & 101.30 \\
\hline FO-15 & HU & h1o & 1 & W-227880 & c & $\mathrm{DAC}$ & 46.90 & 14.20 & 3.10 & 9.50 & 10.00 & 11.10 & 2.30 & .75 & .28 & 1.80 & .22 & .17 & .02 & 100.34 \\
\hline FO-16 & ML & k2 & 1 & W-227875 & c & $\mathrm{RBM}+\mathrm{DAC}$ & 49.40 & 11.80 & 1.90 & 8.80 & 12.90 & 9.10 & 1.90 & .22 & .29 & 1.80 & .30 & .15 & .01 & 98.57 \\
\hline FO-18 & ML & $k 2$ & 1 & D-313518 & b & JPL & 52.00 & 13.20 & 11.20 & nd & 9.24 & 10.50 & 2.14 & .32 & .00 & 2.15 & .22 & .16 & nd & 101.13 \\
\hline FO-19 & ML & $k 2$ & 1 & W-227879 & c & RBM & 48.10 & 10.00 & 1.70 & 10.00 & 18.60 & 7.50 & 2.60 & .19 & .29 & 1.60 & .20 & .18 & .04 & 101.00 \\
\hline FO-20 & $\mathrm{HU}$ & hc1o & 1 & W-227877 & c & $\mathrm{RBM}+\mathrm{DAC}$ & 45.70 & 13.90 & 4.70 & 7.70 & 10.00 & 10.30 & 2.60 & .70 & .26 & 2.20 & .38 & .19 & .01 & 98.64 \\
\hline
\end{tabular}


Table 1.-Chemical analyses-Continued

\begin{tabular}{|c|c|c|c|c|c|c|c|c|c|c|c|c|c|c|c|c|c|c|c|c|}
\hline $\begin{array}{c}\text { Sample } \\
\text { locality } \\
\text { No. }\end{array}$ & $\begin{array}{l}\text { Vol- } \\
\text { cano }\end{array}$ & Unit & $\begin{array}{l}\text { Map } \\
\text { sheet } \\
\text { No. }\end{array}$ & $\begin{array}{l}\text { Laboratory } \\
\text { No. }\end{array}$ & Remark & Collector & $\mathrm{SiO}_{2}$ & $\mathrm{Al}_{2} \mathrm{O}_{3}$ & $\mathrm{Fe}_{2} \mathrm{O}_{3}$ & $\mathrm{FeO}$ & $\mathrm{MgO}$ & $\mathrm{CaO}$ & $\mathrm{Na}_{2} \mathrm{O}$ & $\mathrm{K}_{2} \mathrm{O}$ & $\mathrm{H}_{2} \mathrm{O}$ & $\mathrm{TiO}_{2}$ & $\mathrm{P}_{2} \mathrm{O}_{5}$ & $\mathrm{MnO}$ & $\mathrm{CO}_{2}$ & Total \\
\hline FO-21 & $\mathrm{ML}$ & kc1y & 1 & W-227876 & c & DAC & 51.50 & 14.30 & 5.50 & 6.00 & 7.60 & 10.40 & 2.40 & .42 & .35 & 2.10 & .37 & .16 & .02 & 101.12 \\
\hline FO-22 & ML & $\mathrm{k} 3$ & 1 & W-227849 & c & RBM & 51.50 & 13.80 & 1.40 & 9.80 & 7.20 & 10.40 & 2.50 & .37 & .55 & 2.20 & .34 & .17 & .01 & 100.24 \\
\hline FO-23 & $\mathrm{HU}$ & h1y & 1 & W-227872 & c & $\mathrm{RBM}+\mathrm{DAC}$ & 47.20 & 13.80 & 3.80 & 8.40 & 10.00 & 12.10 & 2.20 & .46 & .32 & 1.70 & .28 & .17 & .04 & 100.47 \\
\hline FP-1 & ML & $k 3$ & 1 & D-278177 & b & $\mathrm{CDC}$ & 52.10 & 13.80 & 12.20 & nd & 6.57 & 10.50 & 2.33 & .47 & .00 & 2.28 & .29 & .17 & nd & 100.71 \\
\hline FP-2 & ML & $\mathrm{k} 3$ & 1 & D-278176 & b & $C D C$ & 51.80 & 14.20 & 11.90 & nd & 7.02 & 10.60 & 2.30 & .44 & .00 & 2.11 & .26 & .16 & nd & 100.79 \\
\hline FP-3 & ML & k4 & 1 & D-278158 & b & CDC & 51.90 & 13.90 & 11.90 & nd & 6.80 & 10.80 & 2.26 & .44 & .34 & 2.16 & .27 & .17 & nd & 100.94 \\
\hline FP-5 & ML & k3 & 1 & D-278179 & b & $\mathrm{CDC}$ & 51.40 & 14.50 & 12.40 & nd & 5.76 & 10.50 & 2.39 & .46 & .27 & 2.52 & .31 & .16 & nd & 100.67 \\
\hline FP-6 & ML & $\mathrm{k} 3$ & 1 & D-278181 & b & $\mathrm{CDC}$ & 52.00 & 13.90 & 11.90 & nd & 6.92 & 10.80 & 2.28 & .46 & .00 & 2.35 & .28 & .17 & nd & 101.06 \\
\hline FP-7 & ML & $k 3$ & 1 & D-278180 & b & $\mathrm{CDC}$ & 52.20 & 13.90 & 12.20 & nd & 6.57 & 10.60 & 2.37 & .47 & .00 & 2.28 & .29 & .17 & nd & 101.05 \\
\hline FP-8 & ML & k3 & 1 & D-278171 & b & $C D C$ & 52.00 & 13.80 & 12.50 & nd & 6.46 & 10.50 & 2.31 & .48 & .00 & 2.36 & .30 & .17 & nd & 100.88 \\
\hline FP-9 & ML & $k 2$ & 1 & D-278170 & b & $\mathrm{CDC}$ & 51.90 & 13.90 & 12.30 & nd & 6.39 & 10.50 & 2.36 & .48 & .00 & 2.32 & .30 & .17 & nd & 100.62 \\
\hline FP-10 & $\mathrm{ML}$ & $k 2$ & 1 & D-278172 & b & CDC & 51.70 & 13.80 & 11.90 & nd & 7.05 & 10.70 & 2.26 & .44 & .08 & 2.31 & .29 & .16 & nd & 100.69 \\
\hline FP-11 & ML & $k 2$ & 1 & D-278173 & b & $\mathrm{CDC}$ & 51.60 & 13.60 & 12.10 & nd & 7.49 & 10.60 & 1.99 & .37 & .39 & 2.22 & .26 & .17 & nd & 100.79 \\
\hline FP-12 & $\mathrm{ML}$ & $k 2$ & 1 & D-278156 & b & $\mathrm{CDC}$ & 52.00 & 13.80 & 12.00 & nd & 6.80 & 10.60 & 2.23 & .45 & .24 & 2.22 & .27 & .17 & nd & 100.78 \\
\hline FP-13 & ML & $\mathrm{K} 3$ & 1 & D-278175 & b & $C D C$ & 52.20 & 13.90 & 12.40 & nd & 6.54 & 10.60 & 2.34 & .48 & .00 & 2.35 & .30 & .17 & nd & 101.28 \\
\hline FP-14 & ML & $\mathrm{K} 3$ & 1 & D-278174 & b & $\mathrm{CDC}$ & 51.90 & 13.80 & 12.20 & nd & 6.91 & 10.60 & 2.19 & .44 & .19 & 2.41 & .29 & .17 & nd & 101.10 \\
\hline FP-15 & ML & $k 3$ & 1 & D-278166 & b & CDC & 51.70 & 13.90 & 12.20 & nd & 6.88 & 10.70 & 2.20 & .39 & .31 & 2.15 & .26 & .17 & nd & 100.86 \\
\hline FP-16 & $\mathrm{ML}$ & $k 2$ & 1 & D-278182 & b & $\mathrm{CDC}$ & 51.80 & 13.90 & 12.10 & nd & 6.60 & 10.70 & 2.25 & .43 & .19 & 2.44 & .28 & .17 & nd & 100.86 \\
\hline FP-17 & ML & $k 3$ & 1 & D-278183 & b & $\mathrm{CDC}$ & 51.70 & 13.90 & 11.90 & nd & 7.05 & 10.60 & 2.19 & .44 & .07 & 2.31 & .28 & .16 & nd & 100.60 \\
\hline FP-18 & ML & $\mathrm{k} 3$ & 1 & D-278184 & b & $C D C$ & 51.70 & 13.70 & 11.80 & nd & 6.93 & 10.50 & 2.22 & .44 & .23 & 2.32 & .29 & .16 & nd & 100.29 \\
\hline FP-19 & ML & 12 & 1 & D-278157 & $b$ & $\mathrm{CDC}$ & 50.20 & 12.00 & 12.70 & nd & 11.80 & 9.67 & 1.82 & .28 & .20 & 1.95 & .19 & .17 & nd & 100.98 \\
\hline FP-20 & ML & $\mathrm{K} 3$ & 1 & D-278164 & b & $\mathrm{CDC}$ & 51.80 & 13.80 & 11.90 & nd & 7.09 & 10.70 & 2.19 & .45 & .00 & 2.34 & .27 & .16 & nd & 100.70 \\
\hline FP-21 & ML & $k 2$ & 1 & D-278162 & b & $\mathrm{CDC}$ & 50.10 & 11.30 & 12.20 & nd & 13.80 & 9.02 & 1.77 & .33 & .09 & 2.02 & .22 & .17 & nd & 101.02 \\
\hline FP-23 & ML & $\mathrm{k} 3$ & 1 & D-278195 & b & $\mathrm{CDC}$ & 52.20 & 13.90 & 12.00 & nd & 6.80 & 10.80 & 2.22 & .45 & .30 & 2.21 & .26 & .17 & nd & 101.31 \\
\hline FP-24 & ML & $k 2$ & 1 & D-278165 & b & CDC & 50.20 & 12.00 & 11.90 & nd & 12.30 & 9.00 & 1.66 & .28 & .86 & 2.11 & .21 & .16 & nd & 100.68 \\
\hline FP-25 & ML & K3 & 1 & D-278168 & $b$ & CDC & 51.70 & 13.80 & 11.90 & nd & 7.12 & 10.60 & 2.17 & .40 & .00 & 2.29 & .27 & .16 & nd & 100.41 \\
\hline FP-26 & ML & $\mathrm{k} 3$ & 1 & D-278167 & b & $\mathrm{CDC}$ & 52.10 & 13.60 & 12.20 & nd & 7.17 & 10.60 & 2.22 & .44 & .32 & 2.23 & .28 & .17 & nd & 101.33 \\
\hline FP-27 & ML & K3 & 1 & D-278185 & $b$ & CDC & 51.90 & 13.30 & 12.10 & nd & 7.65 & 10.70 & 2.07 & .39 & .08 & 2.20 & .25 & .17 & nd & 100.81 \\
\hline FP-28 & ML & $\mathrm{k} 3$ & 1 & D-278159 & $b$ & CDC & 52.20 & 13.80 & 12.00 & nd & 6.84 & 10.70 & 2.30 & .44 & .05 & 2.21 & .28 & .17 & nd & 100.99 \\
\hline FP-29 & ML & $k 3$ & 1 & D-278186 & b & $C D C$ & 52.00 & 13.80 & 11.90 & nd & 6.97 & 10.70 & 2.19 & .45 & .00 & 2.32 & .27 & .16 & nd & 100.76 \\
\hline FP-30 & ML & $k 2$ & 1 & D-278169 & b & CDC & 51.80 & 13.90 & 12.10 & nd & 7.26 & 10.70 & 2.22 & .43 & .00 & 2.37 & .28 & .17 & nd & 101.23 \\
\hline FP-31 & ML & $k 2$ & 1 & D-278187 & $\mathrm{b}$ & $C D C$ & 49.40 & 11.30 & 11.70 & nd & 13.50 & 8.73 & 1.75 & .30 & 1.64 & 1.87 & .25 & .16 & nd & 100.60 \\
\hline FP-32 & ML & $k 2$ & 1 & D-278188 & $b$ & CDC & 49.60 & 10.60 & 12.20 & nd & 15.70 & 8.57 & 1.64 & .26 & .21 & 1.76 & .18 & .17 & nd & 100.89 \\
\hline FP-33 & ML & k3 & 1 & D-278121 & b & CDC & 52.20 & 13.90 & 12.10 & nd & 6.96 & 10.70 & 2.25 & .46 & .00 & 2.39 & .28 & .17 & nd & 101.41 \\
\hline FP-34 & ML & $\mathrm{k} 3$ & 1 & D-278122 & b & CDC & 51.90 & 13.90 & 12.10 & nd & 6.76 & 10.60 & 2.20 & .46 & .00 & 2.44 & .28 & .17 & nd & 100.81 \\
\hline FP-35 & ML & $\mathbf{k} 3$ & 1 & D-278189 & $b$ & CDC & 51.40 & 13.90 & 12.20 & nd & 6.87 & 10.70 & 2.16 & .38 & .00 & 2.11 & .26 & .17 & nd & 100.15 \\
\hline FP-36 & ML & K3 & 1 & D-278161 & b & $\mathrm{CDC}$ & 50.30 & 11.90 & 12.30 & nd & 11.70 & 9.73 & 1.84 & .29 & .04 & 1.96 & .20 & .17 & nd & 100.43 \\
\hline FP-37 & ML & k3 & 1 & D-278191 & b & CDC & 52.00 & 13.70 & 12.30 & nd & 7.01 & 10.60 & 2.24 & .44 & .00 & 2.25 & .29 & .17 & nd & 101.00 \\
\hline FP-38 & ML & $\mathrm{k} 3$ & 1 & D-278190 & b & CDC & 51.80 & 13.80 & 11.90 & nd & 6.91 & 10.70 & 2.26 & .45 & .00 & 2.31 & .27 & .17 & nd & 100.57 \\
\hline PP-39 & ML & k3 & 1 & D-278194 & b & CDC & 52.00 & 13.80 & 11.90 & nd & 7.02 & 10.70 & 2.17 & .45 & .09 & 2.32 & .28 & .16 & nd & 100.89 \\
\hline
\end{tabular}


Table 1.-Chemical analyses-Continued

\begin{tabular}{|c|c|c|c|c|c|c|c|c|c|c|c|c|c|c|c|c|c|c|c|c|}
\hline $\begin{array}{c}\text { Sample } \\
\text { locality } \\
\text { No. }\end{array}$ & $\begin{array}{l}\text { Vol- } \\
\text { cano }\end{array}$ & Unit & $\begin{array}{c}\text { Map } \\
\text { sheet } \\
\text { No. }\end{array}$ & $\begin{array}{l}\text { Laboratory } \\
\text { No. }\end{array}$ & Remark & Collector & $\mathrm{SiO}_{2}$ & $\mathrm{Al}_{2} \mathrm{O}_{3}$ & $\mathrm{Fe}_{2} \mathrm{O}_{3}$ & $\mathrm{FeO}$ & $\mathrm{MgO}$ & $\mathrm{CaO}$ & $\mathrm{Na}_{2} \mathrm{O}$ & $\mathrm{K}_{2} \mathrm{O}$ & $\mathrm{H}_{2} \mathrm{O}$ & $\mathrm{TiO}_{2}$ & $\mathrm{P}_{2} \mathrm{O}_{5}$ & $\mathrm{MnO}$ & $\mathrm{CO}_{2}$ & Total \\
\hline FP-40 & ML & k4 & 1 & D-278160 & b & $\mathrm{CDC}$ & 52.10 & 13.70 & 11.90 & nd & 7.03 & 10.70 & 2.24 & .43 & .27 & 2.19 & .27 & .17 & nd & 101.00 \\
\hline FP-41 & ML & k3 & 1 & D-276100 & b & EWW & 51.90 & 13.80 & 12.40 & nd & 7.28 & 10.80 & 2.28 & .39 & .00 & 2.13 & .25 & .17 & nd & 101.40 \\
\hline FP-42 & ML & $k 3$ & 1 & D-278123 & b & EWW & 52.10 & 13.80 & 11.90 & nd & 7.21 & 10.80 & 2.23 & .44 & .00 & 2.29 & .27 & .16 & nd & 101.20 \\
\hline FP-43 & ML & $k 3$ & 1 & D-278120 & b & $C D C$ & 52.20 & 13.70 & 12.30 & nd & 7.30 & 10.60 & 2.34 & .46 & .00 & 2.26 & .28 & .17 & nd & 101.61 \\
\hline FP-44 & ML & k1y & 1 & D-276102 & b & EWW & 50.40 & 11.80 & 12.00 & nd & 12.50 & 9.54 & 1.88 & .32 & .00 & 2.04 & .21 & .17 & nd & 100.86 \\
\hline FP-45 & ML & $k 2$ & 1 & D-278193 & b & $\mathrm{CDC}$ & 50.00 & 11.00 & 12.10 & nd & 14.70 & 8.72 & 1.81 & .29 & .00 & 1.83 & .19 & .16 & nd & 100.80 \\
\hline FP-46 & ML & $k 3$ & 1 & D-278192 & b & $C D C$ & 50.80 & 12.40 & 11.60 & nd & 11.30 & 9.61 & 1.95 & .31 & .21 & 2.03 & .22 & .16 & nd & 100.59 \\
\hline FP-47 & ML & k2 & 1 & D-276101 & b & EWW & 51.70 & 13.70 & 12.30 & nd & 7.24 & 10.70 & 2.30 & .39 & .00 & 2.12 & .26 & .17 & nd & 100.88 \\
\hline FP-48 & ML & k2 & 1 & D-276103 & b & EWW & 51.20 & 13.90 & 12.40 & nd & 6.88 & 10.40 & 2.15 & .38 & .53 & 2.18 & .27 & .18 & nd & 100.47 \\
\hline FP-49 & ML & $k 2$ & 1 & D-276854 & b & EWW & 51.70 & 13.80 & 12.30 & nd & 7.01 & 10.80 & 2.19 & .38 & .00 & 2.13 & .26 & .17 & nd & 100.74 \\
\hline FP-50 & ML & k2 & 1 & D-278124 & b & EWW & 52.00 & 13.60 & 12.50 & nd & 6.90 & 10.90 & 2.20 & .41 & .00 & 2.45 & .26 & .17 & nd & 101.39 \\
\hline FP-51 & ML & $k 3$ & 1 & D-278125 & b & EWW & 51.60 & 12.90 & 11.70 & nd & 9.91 & 10.10 & 2.13 & .39 & .00 & 2.37 & .25 & .16 & nd & 101.51 \\
\hline FP-52 & ML & k1y & 1 & D-276855 & b & EWW & 50.10 & 12.00 & 11.90 & nd & 12.10 & 9.61 & 1.83 & .32 & .00 & 2.08 & .21 & .16 & nd & 100.31 \\
\hline FP-53 & ML & $\mathrm{k} 2$ & 1 & D-276853 & b & EWW & 51.70 & 13.80 & 12.30 & nd & 7.24 & 10.70 & 2.18 & .38 & .00 & 2.13 & .25 & .17 & nd & 100.85 \\
\hline FQ-1 & ML & k3 & 1 & D-313837 & b & JPL & 51.90 & 14.00 & 12.10 & nd & 6.94 & 10.90 & 2.27 & .43 & .00 & 2.13 & .27 & .17 & nd & 101.11 \\
\hline FQ-2 & ML & k3 & 1 & D-313810 & $\mathrm{b}$ & JPL & 52.00 & 13.80 & 12.40 & nd & 7.00 & 10.60 & 2.34 & .46 & nd & 2.41 & .29 & .17 & nd & 101.47 \\
\hline FQ-4 & ML & kc4 & 1 & D-313492 & $b$ & JPL & 52.00 & 13.90 & 12.10 & nd & 6.95 & 10.80 & 2.33 & .45 & .00 & 2.23 & .29 & .17 & nd & 101.22 \\
\hline FQ-5 & ML & kc4 & 1 & NE-37 & a & JMR & 52.02 & 13.64 & 11.80 & nd & 6.98 & 10.09 & 2.37 & .41 & nd & 2.04 & .25 & .16 & nd & 99.76 \\
\hline FQ-6 & ML & kc3 & 1 & NE-36 & a & JMR & 52.11 & 13.88 & 11.86 & nd & 6.83 & 10.44 & 1.98 & .44 & nd & 2.11 & .27 & .17 & nd & 100.09 \\
\hline FQ-7 & ML & kc5-1935 & 1 & ML-9 & a & JPL & 51.35 & 13.53 & 11.97 & nd & 7.03 & 10.51 & 2.08 & .36 & nd & 2.09 & .23 & .16 & nd & 99.31 \\
\hline FQ-8 & ML & k5-1942 & 1 & ML-11 & a & JPL & 51.79 & 13.63 & 12.07 & nd & 7.05 & 10.52 & 2.25 & .37 & nd & 2.06 & .25 & .16 & nd & 100.15 \\
\hline FQ-9 & ML & kc5-1899 & 1 & ML-38 & a & JMR & 51.61 & 13.61 & 12.10 & nd & 6.93 & 10.54 & 2.14 & .35 & nd & 2.09 & .23 & .17 & nd & 99.77 \\
\hline $\mathrm{FQ}-10$ & ML & k5-1935 & 1 & ML-39 & a & JMR & 51.59 & 13.68 & 12.09 & nd & 6.97 & 10.53 & 2.17 & .37 & nd & 2.06 & .23 & .17 & nd & 99.86 \\
\hline FQ-11 & ML & k5-1975 & 1 & D-313804 & b & JPL & 51.90 & 13.90 & 12.60 & nd & 6.70 & 10.80 & 2.28 & .38 & .00 & 2.18 & .25 & .18 & nd & 101.17 \\
\hline $\mathrm{FQ}-12$ & ML & k4 & 1 & D-313799 & $\mathrm{b}$ & JPL & 51.90 & 14.10 & 12.10 & nd & 6.83 & 10.90 & 2.28 & .39 & .00 & 2.14 & .26 & .17 & nd & 101.07 \\
\hline $\mathrm{FQ}-13$ & ML & k4 & 1 & NE-63 & a & JPL & 51.70 & 13.77 & 11.70 & nd & 6.60 & 10.23 & 2.50 & .40 & nd & 2.06 & .27 & .18 & nd & 99.41 \\
\hline FQ-14 & ML & k5-1852 & 1 & D-313802 & b & JPL & 51.20 & 13.90 & 12.00 & nd & 7.01 & 10.80 & 2.28 & .42 & .00 & 2.20 & .27 & .17 & nd & 100.25 \\
\hline FQ-15 & ML & k5-1942 & 1 & D-313801 & b & JPL & 51.60 & 13.70 & 12.40 & nd & 7.17 & 10.80 & 2.23 & .36 & .00 & 2.17 & .24 & .17 & nd & 100.84 \\
\hline FQ-16 & ML & k4 & 1 & D-313805 & b & JPL & 52.10 & 14.00 & 12.00 & nd & 6.81 & 10.80 & 2.30 & .46 & .00 & 2.24 & .29 & .17 & nd & 101.17 \\
\hline FQ-17 & ML & k5-1935 & 1 & ML-36 & a & JMR & 51.76 & 13.64 & 12.10 & nd & 7.03 & 10.59 & 2.41 & .38 & nd & 2.06 & .22 & .17 & nd & 100.36 \\
\hline FQ-18 & ML & k4 & 1 & D-313809 & b & JPL & 51.90 & 13.90 & 12.10 & nd & 7.30 & 10.80 & 2.21 & .40 & .00 & 2.08 & .26 & .17 & nd & 101.12 \\
\hline FQ-19 & ML & $k 2$ & 1 & D-313810 & $\mathrm{b}$ & JPL & 52.00 & 13.80 & 12.40 & nd & 7.00 & 10.60 & 2.34 & .46 & .00 & 2.41 & .29 & .17 & nd & 101.47 \\
\hline $\mathrm{FQ}-20$ & ML & $k 5-1843$ & 1 & D-313803 & b & JPL & 51.90 & 13.90 & 12.30 & nd & 6.94 & 10.70 & 2.34 & .46 & .00 & 2.22 & .29 & .17 & nd & 101.22 \\
\hline FQ-21 & ML & k5-1935 & 1 & ML-35 & a & JMR & 51.38 & 13.58 & 12.13 & nd & 7.06 & 10.59 & 2.19 & .36 & nd & 2.07 & .22 & .17 & nd & 99.75 \\
\hline FQ-22 & ML & k5-1935 & 1 & ML-34 & a & JMR & 51.30 & 13.54 & 12.05 & nd & 6.99 & 10.62 & 2.23 & .36 & nd & 2.06 & .22 & .17 & nd & 99.54 \\
\hline $\mathrm{FQ}-23$ & ML & k5-1899 & 1 & D-313806 & b & JPL & 51.80 & 13.70 & 12.40 & nd & 7.12 & 10.90 & 2.21 & .34 & .00 & 2.16 & .24 & .17 & nd & 101.04 \\
\hline FQ-24 & ML & k4 & 1 & NE-29 & a & JMR & 51.68 & 13.77 & 11.75 & nd & 6.88 & 10.55 & 1.98 & .40 & nd & 2.05 & .26 & .17 & nd & 99.49 \\
\hline FQ-25 & ML & $k 4$ & 1 & D-313800 & $b$ & JPL & 52.10 & 13.80 & 12.10 & nd & 6.85 & 10.80 & 2.35 & .40 & .00 & 2.11 & .25 & .17 & nd & 100.93 \\
\hline FQ-26 & ML. & k3 & 1 & D-313807 & b & JPL & 50.90 & 12.30 & 11.90 & nd & 11.10 & 9.85 & 2.01 & .35 & .00 & 2.20 & .24 & .16 & nd & 101.01 \\
\hline FQ-27 & ML & k3 & 1 & D-313808 & b & JPL & 51.80 & 13.50 & 12.70 & nd & 6.70 & 11.00 & 2.08 & .40 & .01 & 2.44 & .26 & .18 & nd & 101.07 \\
\hline
\end{tabular}


Table 1.-Chemical analyses-Continued

\begin{tabular}{|c|c|c|c|c|c|c|c|c|c|c|c|c|c|c|c|c|c|c|c|c|}
\hline $\begin{array}{c}\text { Sample } \\
\text { locality } \\
\text { No. }\end{array}$ & $\begin{array}{l}\text { Vol- } \\
\text { cano }\end{array}$ & Unit & $\begin{array}{c}\text { Map } \\
\text { sheet } \\
\text { No. }\end{array}$ & $\begin{array}{l}\text { Laboratory } \\
\text { No. }\end{array}$ & Remark & Collector & $\mathrm{SiO}_{2}$ & $\mathrm{Al}_{2} \mathrm{O}_{3}$ & $\mathrm{Fe}_{2} \mathrm{O}_{3}$ & $\mathrm{FeO}$ & $\mathrm{MgO}$ & $\mathrm{CaO}$ & $\mathrm{Na}_{2} \mathrm{O}$ & $\mathrm{K}_{2} \mathrm{O}$ & $\mathrm{H}_{2} \mathrm{O}$ & $\mathrm{TiO}_{2}$ & $\mathrm{P}_{2} \mathrm{O}_{5}$ & $\mathrm{MnO}$ & $\mathrm{CO}_{2}$ & Total \\
\hline FQ-28 & ML & $\mathrm{k} 3$ & 1 & D-313798 & $\mathrm{b}$ & JPL & 51.90 & 13.90 & 11.40 & nd & 7.45 & 10.70 & 2.30 & .42 & .00 & 2.47 & .27 & .10 & nd & 100.91 \\
\hline FQ-29 & ML & $k 3$ & 1 & D-313812 & b & JPL & 50.80 & 12.70 & 12.40 & nd & 9.70 & 10.30 & 2.07 & .38 & .00 & 2.21 & .25 & .17 & nd & 100.98 \\
\hline FQ-30 & ML & k1y & 1 & D-313813 & b & JPL & 47.90 & 9.94 & 12.10 & nd & 18.70 & 8.44 & 1.55 & .23 & .00 & 1.27 & .16 & .16 & nd & 100.45 \\
\hline FQ-31 & ML & kc4 & 1 & NE-10 & $a$ & JPL & 51.10 & 13.71 & 4.10 & 6.81 & 6.61 & 10.11 & 2.52 & .41 & nd & 2.06 & .26 & .17 & nd & 97.86 \\
\hline FR-1 & ML & $\mathrm{k} 4$ & 1 & D-313832 & b & JPL & 52.20 & 13.50 & 12.90 & nd & 6.23 & 10.40 & 2.45 & .51 & .00 & 2.50 & .32 & .18 & nd & 101.19 \\
\hline FR-2 & ML & $k 3$ & 1 & D-313831 & b & JPL & 51.50 & 13.10 & 12.70 & nd & 8.02 & 10.30 & 2.16 & .47 & .00 & 2.30 & .30 & .18 & nd & 101.03 \\
\hline FR-3 & ML & k3 & 1 & NE-43 & a & JMR & 51.32 & 13.48 & 12.45 & nd & 5.97 & 10.00 & 2.64 & .53 & nd & 2.55 & .33 & .18 & nd & 99.45 \\
\hline FR-4 & ML. & $\mathrm{K} 3$ & 1 & NE-42 & a & JMR & 48.67 & 10.31 & 11.91 & nd & 16.69 & 8.11 & 1.83 & .35 & nd & 1.58 & .23 & .17 & nd & 99.85 \\
\hline FR-5 & ML & k4 & 1 & D-313830 & b & JPL & 52.10 & 13.60 & 12.30 & nd & 7.19 & 10.80 & 2.23 & .43 & .00 & 2.15 & .27 & .17 & nd & 101.24 \\
\hline FR-6 & ML & $k 5-1880-81$ & 1 & ML-48 & a & JMR & 51.87 & 13.21 & 11.97 & nd & 7.79 & 10.14 & 2.14 & .35 & nd & 1.96 & .21 & .17 & nd & 99.81 \\
\hline FR-7 & ML & $\mathrm{k} 4$ & 1 & D-313829 & b & JPL & 52.10 & 13.70 & 12.10 & nd & 6.83 & 10.70 & 2.29 & .44 & .00 & 2.22 & .27 & .16 & nd & 100.81 \\
\hline FR-9 & ML & $k 5-1880-81$ & 1 & ML-45 & $\mathrm{a}$ & JMR & 51.94 & 13.26 & 12.00 & nd & 7.75 & 10.13 & 2.13 & .34 & nd & 1.96 & .21 & .17 & nd & 99.89 \\
\hline FR-10 & ML & k5-1855 & 1 & ML-46 & $\mathbf{a}$ & JMR & 50.98 & 13.30 & 12.10 & nd & 8.27 & 10.21 & 2.28 & .41 & nd & 2.07 & .25 & .17 & nd & 100.04 \\
\hline FR-12 & ML & kcto & 1 & NE-44 & a & JPL & 51.63 & 13.37 & 12.31 & nd & 6.00 & 9.91 & 2.63 & .49 & nd & 2.38 & .32 & .19 & nd & 99.23 \\
\hline FR-14 & ML & k4 & 1 & NE-39 & a & JMR & 51.84 & 13.25 & 12.35 & nd & 5.97 & 9.83 & 2.44 & .49 & nd & 2.42 & .32 & .17 & nd & 99.08 \\
\hline FR-16 & ML & $\mathrm{kc3}$ & 1 & NE-40 & a & JMR & 48.29 & 10.25 & 11.78 & nd & 16.09 & 8.00 & 1.91 & .35 & nd & 1.56 & .23 & .18 & nd & 98.64 \\
\hline FR-17 & ML & k4 & 1 & NE-33 & $\mathbf{a}$ & JMR & 51.95 & 13.79 & 11.80 & nd & 7.07 & 10.53 & 1.99 & .42 & nd & 2.06 & .26 & .17 & nd & 100.04 \\
\hline FR-18 & ML & k4 & 1 & NE-34 & a & JMR & 51.89 & 13.87 & 11.85 & nd & 6.97 & 10.40 & 2.01 & .43 & nd & 2.08 & .28 & .17 & nd & 99.95 \\
\hline FR-19 & ML & k4 & 1 & NE-32A & a & JMR & 51.76 & 13.09 & 12.25 & nd & 7.51 & 9.99 & 2.01 & .49 & nd & 2.24 & .30 & .18 & nd & 99.82 \\
\hline FR-20 & ML & $k 5-1880-81$ & 1 & $M L-49$ & a & JMR & 51.79 & 13.37 & 11.91 & nd & 7.62 & 10.20 & 2.13 & .34 & nd & 1.98 & .20 & .17 & nd & 99.71 \\
\hline FR-21 & ML & k5-1855 & 1 & ML-51 & a & JMR & 51.45 & 13.77 & 11.69 & nd & 7.28 & 10.48 & 2.05 & .43 & nd & 2.11 & .26 & .16 & nd & 99.68 \\
\hline FR-24 & ML & k5-1880-81 & 1 & ML-132 & a & JMR & 52.05 & 13.35 & 11.89 & nd & 7.38 & 10.26 & 2.13 & .34 & nd & 1.99 & .21 & .17 & nd & 99.77 \\
\hline FR-25 & ML & kc1y & 1 & NE-32B & a & JMR & 48.66 & 10.95 & 12.33 & nd & 15.35 & 8.44 & 1.51 & .31 & nd & 1.70 & .22 & .17 & nd & 99.64 \\
\hline FR-27 & ML & k5-1942 & 1 & ML-37 & a & JMR & 51.34 & 13.58 & 12.13 & nd & 7.07 & 10.52 & 2.18 & .36 & nd & 2.05 & .22 & .17 & nd & 99.62 \\
\hline FR-29 & ML & k5-1899 & 1 & D-313826 & b & JPL & 51.80 & 13.60 & 12.40 & nd & 7.13 & 10.90 & 2.21 & .35 & .00 & 2.16 & .24 & .17 & nd & 100.96 \\
\hline FR-31 & ML & k5-1880-81 & 1 & ML-33 & $\mathbf{a}$ & JMR & 51.84 & 13.52 & 11.87 & nd & 7.50 & 10.38 & 2.40 & .35 & nd & 1.99 & .21 & .17 & nd & 100.23 \\
\hline FR-32 & ML & k5-1855 & 1 & ML-29 & a & JMR & 50.46 & 12.86 & 12.19 & nd & 9.08 & 9.94 & 1.99 & .40 & nd & 2.03 & .24 & .16 & nd & 99.35 \\
\hline FR-33 & ML & $k 2$ & 1 & D-313828 & b & $J P L$ & 51.90 & 13.80 & 12.50 & nd & 6.93 & 10.70 & 2.21 & .38 & .00 & 2.13 & .26 & .17 & nd & 100.98 \\
\hline FR-35 & ML & $k 4$ & 1 & D-313827 & $\mathrm{b}$ & JPL & 51.90 & 13.80 & 12.10 & nd & 7.16 & 10.80 & 2.25 & .39 & .00 & 2.10 & .26 & .17 & nd & 100.93 \\
\hline FR-36 & ML & $k 5-1880-81$ & 1 & ML-30 & a & JMR & 52.09 & 13.48 & 11.94 & nd & 7.55 & 10.23 & 2.17 & .37 & nd & 2.00 & .22 & .17 & nd & 100.22 \\
\hline FR-37 & ML & k5-1852 & 1 & ML-31 & a & JMR & 48.68 & 10.64 & 12.25 & nd & 16.06 & 8.17 & 1.70 & .34 & nd & 1.66 & .20 & .15 & nd & 99.85 \\
\hline FS-1 & ML & k1o & 2 & W-214677 & c & JPL & 49.60 & 10.00 & 1.70 & 9.90 & 16.00 & 7.90 & 2.10 & .28 & .59 & 1.50 & .20 & .20 & .00 & 99.97 \\
\hline FS-2 & ML & $\mathrm{kc}$ & 2 & W-214676 & c & JPL & 51.70 & 12.90 & 2.10 & 9.10 & 8.90 & 9.80 & 2.20 & .42 & .84 & 2.00 & .29 & .19 & .00 & 100.44 \\
\hline FS-4 & ML & $\mathrm{k} 3$ & 2 & D-313528 & b & JPL & 52.70 & 13.10 & 12.50 & nd & 7.20 & 10.60 & 2.14 & .35 & .00 & 2.16 & .22 & .17 & nd & 101.14 \\
\hline FS-5 & ML & kc1o & 2 & W-228252 & $\mathrm{b}$ & JPL & 48.10 & 14.30 & 15.40 & nd & 5.84 & 9.66 & 2.57 & .48 & .61 & 3.00 & .35 & .20 & nd & 100.51 \\
\hline FS-6 & ML & k4 & 2 & NE-54 & a & JMR & 51.50 & 13.80 & 2.90 & 8.70 & 7.50 & 10.20 & 2.40 & .51 & .40 & 2.20 & .36 & .19 & nd & 100.66 \\
\hline FS-7 & ML & kc1o & 2 & NE-11 & a & JMR & 49.19 & 14.51 & 14.34 & nd & 5.73 & 8.95 & 2.30 & .53 & nd & 3.07 & .36 & .20 & nd & 99.18 \\
\hline FS-8 & ML & k2 & 2 & W-228251 & $\mathrm{b}$ & JPL & 51.10 & 12.80 & 13.10 & nd & 9.32 & 10.20 & 2.03 & .36 & .00 & 1.94 & .23 & .18 & nd & 101.26 \\
\hline FS-9 & ML & $k 2$ & 2 & NE-47 & a & JMR & 51.62 & 13.52 & 11.87 & nd & 6.97 & 10.39 & 2.45 & .41 & nd & 2.17 & .27 & .17 & nd & 99.84 \\
\hline FS-11 & ML & $k 10$ & 2 & D-313529 & b & $\mathrm{JPL}$ & 51.80 & 13.70 & 12.20 & nd & 7.23 & 10.70 & 2.21 & .40 & .15 & 2.15 & .25 & .17 & nd & 100.96 \\
\hline
\end{tabular}


Table 1.-Chemical analyses-Continued

\begin{tabular}{|c|c|c|c|c|c|c|c|c|c|c|c|c|c|c|c|c|c|c|c|c|}
\hline $\begin{array}{c}\text { Sample } \\
\text { locality } \\
\text { No. }\end{array}$ & $\begin{array}{l}\text { Vol- } \\
\text { cano }\end{array}$ & Unit & $\begin{array}{l}\text { Map } \\
\text { sheet } \\
\text { No. }\end{array}$ & $\begin{array}{l}\text { Laboratory } \\
\text { No. }\end{array}$ & Remark & Collector & $\mathrm{SiO}_{2}$ & $\mathrm{Al}_{2} \mathrm{O}_{3}$ & $\mathrm{Fe}_{2} \mathrm{O}_{3}$ & $\mathrm{FeO}$ & $\mathrm{MgO}$ & $\mathrm{CaO}$ & $\mathrm{Na}_{2} \mathrm{O}$ & $\mathrm{K}_{2} \mathrm{O}$ & $\mathrm{H}_{2} \mathrm{O}$ & $\mathrm{TiO}_{2}$ & $\mathrm{P}_{2} \mathrm{O}_{5}$ & $\mathrm{MnO}$ & $\mathrm{CO}_{2}$ & Total \\
\hline FS-12 & $\mathrm{ML}$ & $\mathrm{kc3}$ & 2 & NE-5 & $\mathrm{a}$ & JMR & 50.41 & 12.83 & 11.70 & nd & 9.79 & 9.23 & 2.10 & .37 & nd & 1.89 & .23 & .17 & nd & 98.72 \\
\hline FS-13 & ML & $k 4$ & 2 & D-313530 & b & JPL & 51.00 & 12.60 & 12.10 & nd & 10.50 & 9.84 & 2.06 & .39 & .00 & 1.97 & .24 & .16 & nd & 100.86 \\
\hline FS-14 & ML & k4 & 2 & NE-6 & a & JMR & 50.72 & 12.46 & 11.85 & nd & 10.87 & 9.46 & 2.10 & .41 & nd & 1.89 & .25 & .17 & nd & 100.18 \\
\hline FS-15 & ML & $k 2$ & 2 & NE-8 & a & JMR & 51.95 & 13.86 & 11.97 & nd & 6.92 & 10.50 & 2.30 & .41 & nd & 2.13 & .25 & .17 & nd & 100.46 \\
\hline FS-16 & ML & k3 & 2 & NE-7 & a & JMR & 52.27 & 13.71 & 11.85 & nd & 7.16 & 10.54 & 2.38 & .39 & nd & 2.05 & .24 & .17 & nd & 100.76 \\
\hline FS-17 & ML & $k 2$ & 2 & W-214670 & c & JPL & 52.30 & 14.10 & 2.60 & 8.60 & 7.10 & 10.40 & 2.50 & .42 & .39 & 2.10 & .31 & .17 & .00 & 100.99 \\
\hline FS-18 & ML & k4 & 2 & D-313531 & b & JPL & 52.10 & 13.70 & 12.00 & nd & 7.38 & 10.70 & 2.29 & .46 & .00 & 2.24 & .28 & .16 & nd & 101.31 \\
\hline FS-19 & ML & $k 2$ & 2 & D-313839 & b & JPL & 51.60 & 13.60 & 12.20 & nd & 7.46 & 10.60 & 2.25 & .41 & .00 & 2.13 & .25 & .17 & nd & 100.67 \\
\hline FS-20 & ML & k2 & 2 & W-214671 & c & JPL & 51.20 & 14.10 & 2.30 & 9.20 & 6.70 & 10.30 & 2.50 & .44 & .53 & 2.10 & .32 & .18 & .00 & 99.87 \\
\hline FS-21 & ML & $\mathrm{k} 3$ & 2 & D-313527 & $b$ & JPL & 52.20 & 13.50 & 12.30 & nd & 7.58 & 10.70 & 2.15 & .39 & .00 & 2.11 & .24 & .17 & nd & 101.34 \\
\hline FS-22 & ML & k5-1942 & 2 & ML-32 & a & JMR & 51.57 & 13.62 & 12.18 & nd & 7.24 & 10.52 & 2.16 & .37 & nd & 2.05 & .23 & .17 & nd & 100.11 \\
\hline FT-1 & ML & $k 3$ & 2 & D-313522 & b & JPL & 52.10 & 13.60 & 12.40 & nd & 6.98 & 10.80 & 2.14 & .35 & .00 & 2.16 & .22 & .17 & nd & 100.92 \\
\hline FT-2 & ML & k & 2 & D-313524 & b & JPL & 51.60 & 13.60 & 11.70 & nd & 8.18 & 10.70 & 2.15 & .37 & .14 & 2.09 & .24 & .16 & nd & 100.93 \\
\hline FT-3 & ML & k10 & 2 & D-313525 & b & JPL & 51.30 & 13.50 & 12.40 & nd & 7.58 & 10.40 & 2.20 & .38 & .37 & 2.26 & .25 & .17 & nd & 100.81 \\
\hline $\mathrm{FT}-4$ & ML & kc1o & 2 & D-313526 & b & JPL & 48.30 & 11.10 & 12.60 & nd & 14.60 & 8.37 & 1.63 & .28 & 1.56 & 1.71 & .20 & .17 & nd & 100.52 \\
\hline FT-5 & $\mathrm{ML}$ & $k 10$ & 2 & D-313523 & b & JPL & 48.00 & 9.85 & 12.70 & nd & 18.90 & 7.89 & 1.58 & .25 & .00 & 1.46 & .17 & .17 & nd & 100.97 \\
\hline FT-6 & ML & k4 & 2 & D-313520 & b & JPL & 52.10 & 14.00 & 11.80 & nd & 6.89 & 10.80 & 2.21 & .45 & .00 & 2.27 & .28 & .16 & nd & 100.96 \\
\hline FT-7 & ML & k3 & 2 & D-313521 & b & JPL & 50.10 & 11.70 & 12.10 & nd & 13.20 & 9.35 & 1.83 & .32 & .00 & 1.72 & .20 & .17 & nd & 100.69 \\
\hline FT-8 & ML & k1o & 2 & D-313519 & b & JPL & 51.00 & 13.50 & 12.50 & nd & 8.00 & 10.60 & 2.17 & .39 & .20 & 2.15 & .25 & .17 & nd & 100.93 \\
\hline FU-1 & $\mathrm{KI}$ & $\mathrm{p} 4$ & 2 & D-306266 & b & FAT & 50.40 & 12.60 & 12.80 & nd & 8.73 & 11.00 & 2.14 & .43 & .00 & 2.55 & .26 & .17 & nd & 101.08 \\
\hline FU-2 & $\mathrm{K}]$ & p4 & 2 & D-306268 & b & FAT & 45.40 & 8.05 & 13.20 & nd & 23.40 & 6.73 & 1.40 & .22 & .00 & 1.41 & .14 & .17 & nd & 100.12 \\
\hline FU-3 & $\mathrm{KI}$ & p4 & 2 & W-213219 & c & RTH & 51.20 & 13.30 & 2.70 & 9.30 & 7.40 & 10.90 & 2.10 & .48 & .21 & 2.50 & .32 & .19 & .02 & 100.62 \\
\hline FU-4 & $\mathrm{KI}$ & p4 & 2 & W-213220 & c & RTH & 50.00 & 13.50 & 2.70 & 8.20 & 7.50 & 10.90 & 2.00 & .49 & .75 & 2.60 & .31 & .19 & .01 & 99.15 \\
\hline FU-5 & $\mathrm{KI}$ & $\mathrm{p} 4$ & 2 & W-213211 & c & RTH & 50.90 & 12.40 & 2.80 & 9.30 & 9.20 & 10.60 & 2.10 & .46 & .59 & 2.30 & .26 & .31 & .01 & 101.23 \\
\hline FU-6 & $\mathrm{KI}$ & p4 & 2 & D-306272 & $b$ & FAT & 51.50 & 13.40 & 12.70 & nd & 7.41 & 10.50 & 2.28 & .42 & .00 & 2.24 & .27 & .17 & nd & 100.89 \\
\hline FU-7 & $\mathrm{KI}$ & $\mathrm{p} 4$ & 2 & W-213221 & c & RTH & 50.50 & 12.90 & 2.70 & 9.10 & 7.80 & 11.00 & 1.90 & .44 & .23 & 2.40 & .29 & .21 & .01 & 99.48 \\
\hline FU-8 & $\mathrm{KI}$ & p4 & 2 & W-213214 & c & RTH & 50.50 & 12.80 & 2.70 & 9.40 & 9.00 & 11.00 & 2.20 & .46 & .25 & 2.40 & .24 & .22 & .01 & 101.18 \\
\hline FU-9 & $\mathrm{KI}$ & p4 & 2 & W-213212 & c & RTH & 50.80 & 12.90 & 2.50 & 9.50 & 8.00 & 10.60 & 2.30 & .50 & .30 & 2.60 & .32 & .24 & .02 & 100.58 \\
\hline FU-10 & $\mathrm{KI}$ & p4 & 2 & W-213213 & c & RTH & 50.20 & 13.50 & 2.30 & 9.60 & 6.60 & 10.10 & 2.40 & .61 & .29 & 2.90 & .36 & .23 & .01 & 99.10 \\
\hline FU-11 & $\mathrm{KI}$ & p4 & 2 & W-213224 & c & RTH & 51.00 & 13.50 & 2.50 & 8.70 & 6.80 & 11.40 & 2.20 & .51 & .15 & 2.50 & .31 & .20 & .01 & 99.78 \\
\hline FU-12 & $\mathrm{KI}$ & $\mathrm{p} 4$ & 2 & D-306273 & $b$ & FAT & 51.00 & 12.90 & 12.60 & nd & 7.84 & 11.60 & 2.09 & .39 & .00 & 2.52 & .25 & .18 & nd & 101.37 \\
\hline FU-13 & KI & p4 & 2 & W-213222 & c & $\mathrm{RTH}$ & 51.50 & 13.30 & 2.40 & 9.30 & 7.20 & 11.50 & 2.00 & .45 & .09 & 2.40 & .29 & .22 & .01 & 100.66 \\
\hline FU-14 & $\mathrm{KI}$ & $\mathrm{p} 4$ & 2 & W-213218 & c & $\mathrm{RTH}$ & 51.30 & 13.60 & 2.40 & 9.00 & 7.40 & 10.80 & 2.20 & .49 & .21 & 2.50 & .29 & .20 & .00 & 100.39 \\
\hline FU-15 & KI & $\mathrm{p} 4$ & 2 & W-213223 & c & RTH & 51.20 & 13.10 & 2.30 & 9.20 & 7.80 & 10.20 & 2.10 & .44 & .48 & 2.30 & .23 & .23 & .01 & 99.59 \\
\hline FU-16 & $\mathrm{KI}$ & p4 & 2 & D-306270 & b & FAT & 49.90 & 12.10 & 13.00 & nd & 10.60 & 10.40 & 2.01 & .34 & .00 & 2.25 & .22 & .18 & nd & 101.00 \\
\hline FU-17 & $\mathrm{KI}$ & $\mathrm{p} 4$ & 2 & D-306267 & b & FAT & 50.80 & 13.20 & 12.30 & nd & 7.84 & 11.40 & 2.18 & .42 & .00 & 2.57 & .25 & .17 & nd & 101.13 \\
\hline FU-18 & $\mathrm{KI}$ & p4 & 2 & W-235734 & c & FAT & 49.40 & 11.70 & 1.80 & 10.00 & 11.60 & 9.50 & 1.90 & .61 & .23 & 2.20 & .25 & .17 & .02 & 99.38 \\
\hline FU-19 & KI & $\mathrm{p} 4$ & 2 & W-213233 & c & RTH & 50.80 & 13.10 & 2.20 & 9.20 & 8.60 & 10.30 & 2.10 & .42 & .31 & 2.30 & .29 & .22 & .00 & 99.84 \\
\hline FU-20 & $\mathrm{KI}$ & p4 & 2 & W-213217 & c & RTH & 50.90 & 13.40 & 2.40 & 8.70 & 7.90 & 10.90 & 2.20 & .49 & .15 & 2.50 & .30 & .20 & .01 & 100.05 \\
\hline FU-21 & $\mathrm{KI}$ & p4 & 2 & W-213225 & c & RTH & 49.70 & 12.80 & 1.80 & 9.80 & 9.60 & 10.40 & 2.10 & .47 & .19 & 2.40 & .28 & .19 & .01 & 99.74 \\
\hline
\end{tabular}


Table 1.-Chemical analyses-Continued

\begin{tabular}{|c|c|c|c|c|c|c|c|c|c|c|c|c|c|c|c|c|c|c|c|c|}
\hline $\begin{array}{c}\text { Sample } \\
\text { locality } \\
\text { No. }\end{array}$ & $\begin{array}{l}\text { Vol- } \\
\text { cano }\end{array}$ & Unit & $\begin{array}{c}\text { Map } \\
\text { sheet } \\
\text { No. }\end{array}$ & $\begin{array}{l}\text { Laboratory } \\
\text { No. }\end{array}$ & Remark & Collector & $\mathrm{SiO}_{2}$ & $\mathrm{Al}_{2} \mathrm{O}_{3}$ & $\mathrm{Fe}_{2} \mathrm{O}_{3}$ & $\mathrm{FeO}$ & $\mathrm{MgO}$ & $\mathrm{CaO}$ & $\mathrm{Na}_{2} \mathrm{O}$ & $\mathrm{K}_{2} \mathrm{O}$ & $\mathrm{H}_{2} \mathrm{O}$ & $\mathrm{TO}_{2}$ & $\mathrm{P}_{2} \mathrm{O}_{5}$ & $\mathrm{MnO}$ & $\mathrm{CO}_{2}$ & Total \\
\hline FU-22 & $\mathrm{K}]$ & p4 & 2 & D-306269 & $\mathrm{b}$ & FAT & 50.80 & 13.10 & 12.30 & nd & 8.46 & 11.20 & 2.16 & .43 & .00 & 2.52 & .25 & .17 & nd & 101.39 \\
\hline FU-23 & KI & p4 & 2 & W-213235 & c & RTH & 50.70 & 13.10 & 1.60 & 9.60 & 8.80 & 10.60 & 2.00 & .43 & .14 & 2.40 & .29 & .22 & .00 & 99.88 \\
\hline FU-24 & $\mathrm{K}$ & $\mathrm{p} 4$ & 2 & D-306271 & b & FAT & 49.70 & 12.20 & 12.70 & nd & 10.90 & 10.40 & 1.98 & .32 & .00 & 2.15 & .21 & .17 & nd & 100.73 \\
\hline FU-25 & KI & p4 & 2 & W-213226 & c & RTH & 51.00 & 13.60 & 3.10 & 8.30 & 7.40 & 10.80 & 2.20 & .49 & .15 & 2.50 & .32 & .17 & .00 & 100.03 \\
\hline FU-26 & $\mathrm{K}]$ & p4 & 2 & D-306265 & b & FAT & 49.60 & 12.10 & 13.00 & nd & 10.50 & 10.40 & 2.04 & .40 & .00 & 2.39 & .23 & .18 & nd & 100.84 \\
\hline FU-29 & ML & k10 & 2 & NE-52 & a & JMR & 47.21 & 9.64 & 12.37 & nd & 19.76 & 7.53 & 1.51 & .22 & nd & 1.33 & .17 & .16 & nd & 99.90 \\
\hline FU-30 & ML & KB & 2 & W-227789 & c & $\mathrm{JBB}$ & 50.50 & 13.50 & 2.50 & 9.70 & 7.50 & 10.00 & 2.30 & .34 & .46 & 2.10 & .29 & .18 & .06 & 99.43 \\
\hline FV-1 & $\mathrm{K}$ & p3 & 2 & W-235747 & c & FAT & 51.10 & 13.80 & 2.10 & 9.20 & 7.20 & 10.80 & 2.40 & .40 & .27 & 2.50 & .30 & .17 & .02 & 100.26 \\
\hline FV-2 & $\mathrm{K}]$ & $\mathrm{p} 4$ & 2 & W-213232 & c & RTH & 50.40 & 12.70 & 2.50 & 9.00 & 9.50 & 10.40 & 2.10 & .44 & .16 & 2.40 & .30 & .20 & .00 & 100.10 \\
\hline FV-3 & KI & p4o & 2 & W-235748 & c & FAT & 51.90 & 14.00 & 3.30 & 8.30 & 6.60 & 10.50 & 2.60 & .50 & .40 & 2.60 & .40 & .17 & .02 & 101.29 \\
\hline FV-4 & $\mathrm{KI}$ & p4 & 2 & W-235745 & c & FAT & 50.30 & 13.10 & 1.70 & 9.40 & 8.50 & 10.90 & 2.10 & .37 & .31 & 2.30 & .25 & .17 & .02 & 99.42 \\
\hline FV-5 & KI & $\mathrm{p} 4$ & 2 & W-235735 & c & FAT & 50.40 & 13.00 & 2.10 & 9.40 & 8.10 & 11.40 & 2.10 & .46 & .20 & 2.30 & .25 & .17 & .02 & 99.90 \\
\hline FV-6 & $\mathrm{K}]$ & p4 & 2 & W-235737 & c & FAT & 50.60 & 12.50 & 1.80 & 10.00 & 9.40 & 10.60 & 2.00 & .45 & .25 & 2.20 & .25 & .18 & .03 & 100.26 \\
\hline FV-7 & KI & p4 & 2 & W-235738 & c & FAT & 50.90 & 13.40 & 2.00 & 9.80 & 7.20 & 10.60 & 2.40 & .48 & .55 & 2.80 & .37 & .17 & .03 & 100.70 \\
\hline FV-8 & $\mathrm{KI}$ & p4 & 2 & W-235740 & c & FAT & 50.70 & 13.30 & 2.80 & 8.50 & 8.10 & 11.20 & 2.10 & .38 & .32 & 2.30 & .26 & .17 & .02 & 100.15 \\
\hline FV-9 & $\mathrm{KI}$ & $\mathrm{p} 4$ & 2 & W-235739 & c & FAT & 50.30 & 12.90 & 3.40 & 8.20 & 8.50 & 10.90 & 2.10 & .37 & .20 & 2.30 & .26 & .17 & .02 & 99.62 \\
\hline FV-10 & KI & p4 & 2 & W-235741 & c & FAT & 50.90 & 13.40 & 2.50 & 8.60 & 7.90 & 11.40 & 2.10 & .35 & .22 & 2.30 & .25 & .17 & .02 & 100.11 \\
\hline FV-11 & KI & p4 & 2 & W-235742 & c & FAT & 49.40 & 12.30 & 2.20 & 9.50 & 10.00 & 10.30 & 2.00 & .34 & .22 & 2.20 & .23 & .17 & .02 & 98.88 \\
\hline GM-1 & $\mathrm{HU}$ & h2 & 1 & W-227907 & c & DAC & 47.10 & 15.40 & 2.90 & 9.60 & 9.10 & 10.60 & 2.80 & .77 & .45 & 2.20 & .32 & .15 & .05 & 101.44 \\
\hline GM-2 & $\mathrm{HU}$ & hiy & 1 & W-227908 & c & DAC & 47.20 & 14.60 & 3.40 & 9.10 & 9.80 & 10.60 & 2.20 & .78 & .38 & 2.10 & .29 & .15 & .03 & 100.63 \\
\hline GM-3 & $\mathrm{HU}$ & hiy & 1 & W-227909 & c & $\mathrm{DAC}$ & 45.60 & 13.90 & 3.60 & 8.60 & 10.40 & 10.70 & 2.40 & .77 & .55 & 2.20 & .34 & .18 & .03 & 99.27 \\
\hline GM-4 & $\mathrm{HU}$ & h2 & 1 & W-227910 & c & DAC & 47.90 & 12.90 & 3.30 & 8.80 & 12.90 & 10.70 & 2.00 & .37 & .48 & 1.60 & .17 & .16 & .04 & 101.32 \\
\hline GM-5 & $\mathrm{HU}$ & h1y & 1 & W-227863 & c & DAC & 46.80 & 14.30 & 3.70 & 9.20 & 9.80 & 9.50 & 2.80 & .80 & .25 & 2.30 & .28 & .18 & .08 & 99.99 \\
\hline GM-6 & $\mathrm{HU}$ & h2 & 1 & W-227864 & c & DAC & 46.70 & 14.40 & 2.80 & 10.00 & 9.40 & 10.40 & 2.50 & .64 & .39 & 2.10 & .32 & .19 & .08 & 99.92 \\
\hline $\mathrm{GN}-1$ & $\mathrm{HU}$ & $\mathrm{h}$ & 1 & W-217501 & c & RBM & 45.90 & 15.50 & 3.20 & 9.70 & 6.00 & 10.50 & 2.90 & .96 & 1.33 & 2.50 & .39 & .20 & .01 & 99.09 \\
\hline GN-2 & $\mathrm{HU}$ & hc & 1 & W-227948 & c & $\mathrm{RBM}+\mathrm{DAC}$ & 48.90 & 15.60 & 5.10 & 7.50 & 6.90 & 11.20 & 2.50 & .70 & .47 & 2.00 & .32 & .19 & .01 & 101.39 \\
\hline GN-3 & $\mathrm{HU}$ & $\mathrm{h}$ & 1 & W-227949 & c & $\mathrm{RBM}+\mathrm{DAC}$ & 48.10 & 15.70 & 6.10 & 7.20 & 5.50 & 10.50 & 3.00 & 1.20 & .46 & 3.00 & .50 & .19 & .00 & 101.45 \\
\hline GN-4 & $\mathrm{HU}$ & h1y & 1 & W-214687 & c & $\mathrm{RBM}$ & 47.70 & 15.20 & 2.60 & 10.08 & 5.40 & 10.10 & 3.20 & 1.27 & .34 & 2.90 & .38 & .20 & .02 & 99.39 \\
\hline GN-5 & $\mathrm{HU}$ & hc1o & 1 & W-214752 & c & RBM & 46.80 & 13.50 & 3.90 & 7.40 & 10.60 & 10.80 & 2.30 & .65 & .34 & 1.80 & .27 & .16 & .02 & 98.54 \\
\hline GN-6 & HU & h1y & 1 & W-217515 & c & $\mathrm{RBM}+\mathrm{DAC}$ & 46.20 & 12.10 & 3.90 & 8.20 & 11.90 & 10.90 & 2.00 & .72 & .93 & 1.90 & .29 & .13 & .02 & 99.19 \\
\hline GN-7 & HU & hc1y & 1 & W-214730 & c & RBM & 46.90 & 14.00 & 5.50 & 5.90 & 10.00 & 11.20 & 2.20 & .63 & .49 & 1.80 & .27 & .15 & .02 & 99.06 \\
\hline GN-8 & $\mathrm{HU}$ & h1y & 1 & W-217516 & c & $\mathrm{RBM}+\mathrm{DAC}$ & 46.10 & 12.90 & 2.60 & 9.60 & 11.20 & 10.10 & 2.30 & .76 & .67 & 1.90 & .25 & .14 & .02 & 98.54 \\
\hline GN-9 & $\mathrm{HU}$ & hc1y & 1 & W-214710 & c & $\mathrm{RBM}$ & 45.00 & 12.50 & 8.10 & 4.32 & 11.10 & 10.20 & 2.10 & .88 & 1.69 & 2.10 & .34 & .20 & .02 & 98.55 \\
\hline GN-10 & $\mathrm{HU}$ & hc & 1 & W-214731 & c & RBM & 47.20 & 15.40 & 2.60 & 10.20 & 6.50 & 10.10 & 3.00 & .85 & .27 & 2.30 & .34 & .17 & .02 & 98.95 \\
\hline GN-11 & $\mathrm{HU}$ & hc1y & 1 & W-214692 & c & RBM & 46.50 & 13.20 & 3.20 & 9.12 & 10.70 & 10.20 & 2.50 & .83 & .18 & 2.00 & .28 & .21 & .02 & 98.94 \\
\hline $\mathrm{GN}-13$ & $\mathrm{HU}$ & h1y & 1 & W-217549 & c & DAC & 49.10 & 15.80 & 3.20 & 8.50 & 4.90 & 9.00 & 3.40 & 1.40 & 1.10 & 2.60 & .50 & .17 & .02 & 99.69 \\
\hline GN-14 & $\mathrm{HU}$ & hc1y & 1 & W-217548 & c & DAC & 46.60 & 15.00 & 5.00 & 8.70 & 8.40 & 10.00 & 2.70 & .91 & .50 & 2.20 & .34 & .19 & .02 & 100.56 \\
\hline GN-15 & $\mathrm{HU}$ & hc10 & 1 & W-217556 & c & DAC & 47.10 & 14.40 & 3.70 & 9.00 & 9.30 & 10.40 & 2.60 & .88 & .17 & 2.30 & .30 & .21 & .07 & 100.43 \\
\hline GN-16 & $\mathrm{HU}$ & hc10 & 1 & W-217553 & c & DAC & 46.80 & 13.80 & 3.30 & 8.60 & 10.20 & 10.90 & 2.20 & .68 & .54 & 1.90 & .26 & .15 & .02 & 99.35 \\
\hline GN-17 & $\mathrm{HU}$ & hc10 & 1 & W-217555 & c & DAC & 46.60 & 15.60 & 3.40 & 7.80 & 9.70 & 11.60 & 2.40 & .59 & .56 & 1.70 & .25 & .18 & .02 & 100.40 \\
\hline
\end{tabular}


Table 1.-Chemical analyses-Continued

\begin{tabular}{|c|c|c|c|c|c|c|c|c|c|c|c|c|c|c|c|c|c|c|c|c|}
\hline $\begin{array}{c}\text { Sample } \\
\text { locality } \\
\text { No. }\end{array}$ & $\begin{array}{l}\text { Vol- } \\
\text { cano }\end{array}$ & Unit & $\begin{array}{c}\text { Map } \\
\text { sheet } \\
\text { No. }\end{array}$ & $\begin{array}{l}\text { Laboratory } \\
\text { No. }\end{array}$ & Remark & Collector & $\mathrm{SiO}_{2}$ & $\mathrm{Al}_{2} \mathrm{O}_{3}$ & $\mathrm{Fe}_{2} \mathrm{O}_{3}$ & $\mathrm{FeO}$ & $\mathrm{MgO}$ & $\mathrm{CaO}$ & $\mathrm{Na}_{2} \mathrm{O}$ & $\mathrm{K}_{2} \mathrm{O}$ & $\mathrm{H}_{2} \mathrm{O}$ & $\mathrm{TOO}_{2}$ & $\mathrm{P}_{2} \mathrm{O}_{5}$ & $\mathrm{MnO}$ & $\mathrm{CO}_{2}$ & Total \\
\hline GN-19 & $\mathrm{HU}$ & h1y & 1 & W-227928 & $\bar{c}$ & $\mathrm{RBM}+\mathrm{DAC}$ & 46.80 & 14.90 & 4.60 & 8.10 & 8.00 & 10.70 & 2.70 & .96 & .16 & 2.30 & .40 & .18 & .01 & 99.81 \\
\hline GN-20 & $\mathrm{HU}$ & hc10 & 1 & W-217550 & c & DAC & 46.50 & 14.00 & 3.30 & 8.90 & 11.00 & 10.20 & 2.50 & .71 & .51 & 1.90 & .27 & .15 & .02 & 99.96 \\
\hline GN-23 & $\mathrm{HU}$ & hc1y & 1 & W-217557 & c & DAC & 46.20 & 14.60 & 4.60 & 8.50 & 9.30 & 10.20 & 2.70 & .90 & .65 & 2.30 & .31 & .20 & .01 & 100.47 \\
\hline $\mathrm{GN}-24$ & $\mathrm{HU}$ & hc1o & 1 & W-227925 & c & $\mathrm{RBM}+\mathrm{DAC}$ & 46.40 & 14.60 & 2.80 & 9.70 & 9.20 & 10.50 & 2.40 & .75 & .55 & 2.10 & .36 & .18 & .02 & 99.56 \\
\hline GN-25 & $\mathrm{HU}$ & hc2 & 1 & W-214753 & c & $\mathrm{RBM}$ & 46.10 & 15.00 & 3.20 & 8.60 & 8.50 & 10.40 & 2.70 & .68 & .28 & 2.10 & .30 & .19 & .02 & 98.07 \\
\hline GN-26 & $\mathrm{HU}$ & h2 & 1 & W-217551 & c & DAC & 46.70 & 13.80 & 2.40 & 10.10 & 10.40 & 10.30 & 2.30 & .76 & .37 & 2.00 & .29 & .14 & .02 & 99.58 \\
\hline GN-27 & $\mathrm{HU}$ & hc2 & 1 & W-227926 & c & $\mathrm{RBM}+\mathrm{DAC}$ & 46.50 & 14.80 & 3.30 & 9.10 & 8.70 & 10.80 & 2.60 & .82 & .23 & 2.10 & .36 & .18 & .03 & 99.52 \\
\hline GN-28 & HU & hc1o & 1 & W-217560 & c & DAC & 46.60 & 14.10 & 2.50 & 9.60 & 10.70 & 10.90 & 2.30 & .70 & .36 & 1.90 & .27 & .16 & .06 & 100.15 \\
\hline GN-32 & HU & h3 & 1 & W-217561 & c & DAC & 47.20 & 14.50 & 2.40 & 10.00 & 9.60 & 10.50 & 2.60 & .84 & .34 & 2.20 & .30 & .17 & .02 & 100.67 \\
\hline GN-33 & $\mathrm{HU}$ & hc3 & 1 & W-214733 & c & RBM & 46.10 & 12.80 & 4.80 & 7.40 & 12.40 & 10.00 & 2.20 & .62 & .81 & 1.80 & .26 & .16 & .02 & 99.37 \\
\hline GN-34 & $\mathrm{HU}$ & ho3 & 1 & W-217519 & c & $\mathrm{RBM}+\mathrm{DAC}$ & 46.30 & 14.10 & 2.60 & 9.50 & 10.30 & 10.10 & 2.50 & .88 & .42 & 2.00 & .31 & .17 & .01 & 99.19 \\
\hline GN-35 & HU & hc1y & 1 & W-217563 & c & DAC & 46.50 & 13.20 & 3.00 & 9.10 & 12.50 & 9.90 & 2.30 & .69 & .33 & 1.90 & .25 & .19 & .01 & 99.87 \\
\hline GN-36 & $\mathrm{HU}$ & hc5-1800-01 & 1 & W-217443 & c & RBM & 46.10 & 14.70 & 5.20 & 7.70 & 8.80 & 9.20 & 2.80 & .92 & .25 & 2.30 & .34 & .17 & .02 & 98.50 \\
\hline GN-37 & $\mathrm{HU}$ & hc3 & 1 & W-217518 & c & $\mathrm{RBM}+\mathrm{DAC}$ & 46.30 & 12.90 & 6.30 & 6.30 & 10.10 & 10.40 & 2.40 & .88 & .46 & 2.00 & .29 & .15 & .01 & 98.49 \\
\hline GN-38 & $\mathrm{HU}$ & hc1o & 1 & W-217521 & c & $\mathrm{RBM}+\mathrm{DAC}$ & 46.10 & 13.10 & 2.20 & 9.80 & 12.30 & 10.40 & 2.20 & .77 & .42 & 1.80 & .29 & .15 & .01 & 99.54 \\
\hline GN-39 & $\mathrm{HU}$ & hc1o & 1 & W-214754 & c & $\mathrm{RBM}$ & 47.80 & 12.60 & 5.70 & 5.80 & 12.30 & 9.80 & 2.00 & .40 & .61 & 1.40 & .18 & .17 & .02 & 98.78 \\
\hline GN-41 & $\mathrm{HU}$ & hc1o & 1 & W-217522 & c & $\mathrm{RBM}+\mathrm{DAC}$ & 47.20 & 15.00 & 9.00 & 2.80 & 7.30 & 11.90 & 2.20 & .78 & .62 & 2.10 & .28 & .15 & .01 & 99.34 \\
\hline GN-42 & $\mathrm{HU}$ & hc2 & 1 & W-217403 & c & $\mathrm{RBM}+\mathrm{DAC}$ & 47.30 & 14.00 & 5.50 & 6.80 & 10.00 & 10.70 & 2.30 & .51 & .33 & 1.70 & .21 & .16 & .01 & 99.52 \\
\hline GN-43 & $\mathrm{HU}$ & hc2 & 1 & W-217406 & c & $\mathrm{RBM}+\mathrm{DAC}$ & 47.00 & 14.60 & 3.90 & 8.60 & 8.70 & 9.80 & 2.80 & .96 & .32 & 2.30 & .37 & .17 & .02 & 99.54 \\
\hline GN-44 & $\mathrm{HU}$ & hc2 & 1 & W-217404 & c & $\mathrm{RBM}+\mathrm{DAC}$ & 46.50 & 14.60 & 7.40 & 5.40 & 9.10 & 9.30 & 2.80 & .86 & .79 & 2.30 & .27 & .16 & .02 & 99.50 \\
\hline GN-45 & $\mathrm{HU}$ & hc2 & 1 & W-217410 & c & $\mathrm{RBM}+\mathrm{DAC}$ & 46.60 & 14.40 & 2.80 & 9.70 & 8.30 & 9.90 & 3.00 & 1.10 & .48 & 2.40 & .39 & .17 & .02 & 99.26 \\
\hline GN-46 & $\mathrm{HU}$ & hc2 & 1 & W-217408 & c & $\mathrm{RBM}+\mathrm{DAC}$ & 46.70 & 14.50 & 4.30 & 8.50 & 8.20 & 9.80 & 2.90 & 1.10 & .43 & 2.50 & .41 & .17 & .01 & 99.52 \\
\hline GN-47 & $\mathrm{HU}$ & hc1o & 1 & W-217405 & c & $\mathrm{RBM}+\mathrm{DAC}$ & 47.20 & 15.10 & 12.70 & .04 & 6.80 & 11.10 & 2.80 & .85 & .39 & 2.30 & .29 & .14 & .01 & 99.72 \\
\hline GN-48 & $\mathrm{HU}$ & hc2 & 1 & W-217409 & c & $\mathrm{RBM}+\mathrm{DAC}$ & 45.50 & 14.00 & 6.80 & 6.30 & 8.80 & 9.60 & 2.70 & .94 & 1.16 & 2.30 & .37 & .17 & .01 & 98.65 \\
\hline GN-50 & $\mathrm{HU}$ & hc1o & 1 & W-217412 & c & $\mathrm{RBM}+\mathrm{DAC}$ & 46.90 & 12.30 & 3.40 & 8.40 & 12.70 & 11.10 & 2.20 & .69 & .38 & 1.70 & .23 & .16 & .01 & 100.17 \\
\hline GN-51 & $\mathrm{HU}$ & hc2 & 1 & W-217417 & c & $\mathrm{RBM}+\mathrm{DAC}$ & 46.70 & 13.70 & 3.00 & 8.80 & 10.10 & 10.60 & 2.60 & .85 & .23 & 2.00 & .29 & .15 & .02 & 99.04 \\
\hline GN-52 & $\mathrm{HU}$ & hc1y & 1 & W-217415 & c & $\mathrm{RBM}+\mathrm{DAC}$ & 45.80 & 13.30 & 4.30 & 8.20 & 12.40 & 10.10 & 2.40 & .68 & .74 & 1.90 & .27 & .14 & .02 & 100.25 \\
\hline GN-53 & $\mathrm{HU}$ & hc1o & 1 & W-217423 & c & $\mathrm{RBM}+\mathrm{DAC}$ & 45.40 & 13.10 & 2.60 & 9.10 & 11.60 & 11.90 & 2.10 & .87 & .47 & 2.00 & .28 & .16 & .02 & 99.60 \\
\hline GN-54 & $\mathrm{HU}$ & hc1o & 1 & W-217420 & c & $\mathrm{RBM}+\mathrm{DAC}$ & 46.70 & 13.30 & 2.50 & 9.10 & 10.90 & 11.20 & 2.30 & .74 & .42 & 1.80 & .25 & .14 & .01 & 99.36 \\
\hline GN-55 & $\mathrm{HU}$ & hc1y & 1 & W-217422 & c & $\mathrm{RBM}+\mathrm{DAC}$ & 45.60 & 13.70 & 5.30 & 7.00 & 12.10 & 10.10 & 2.30 & .73 & .53 & 1.80 & .26 & .14 & .01 & 99.57 \\
\hline GN-56 & HU & h10 & 1 & W-217421 & c & $\mathrm{RBM}+\mathrm{DAC}$ & 47.40 & 13.60 & 4.10 & 7.50 & 10.50 & 11.20 & 2.40 & .77 & .38 & 1.90 & .26 & .15 & .02 & 100.18 \\
\hline GN-57 & $\mathrm{HU}$ & hc1o & 1 & W-217424 & c & RBM+DAC & 44.80 & 12.70 & 2.20 & 9.70 & 12.40 & 11.00 & 2.30 & .97 & .47 & 2.00 & .30 & .14 & .09 & 99.07 \\
\hline GN-58 & $\mathrm{HU}$ & hc1o & 1 & W-227947 & c & $\mathrm{RBM}+\mathrm{DAC}$ & 48.50 & 14.00 & 7.30 & 4.60 & 10.50 & 11.50 & 1.90 & .72 & .41 & 1.40 & .24 & .17 & .01 & 101.25 \\
\hline GO-1 & HU & hc4 & 1 & W-217455 & c & RBM & 46.40 & 14.20 & 2.90 & 9.70 & 9.60 & 9.90 & 2.70 & .82 & .28 & 2.10 & .30 & .19 & .02 & 99.11 \\
\hline GO-2 & ML & k2 & 1 & W-227855 & c & RBM & 50.10 & 11.00 & 1.70 & 9.70 & 15.40 & 8.40 & 1.80 & .19 & .40 & 1.70 & .23 & .17 & .01 & 100.80 \\
\hline GO-4 & HU & hc4 & 1 & W-214734 & c & RBM & 46.30 & 14.30 & 3.00 & 9.40 & 9.20 & 10.00 & 2.80 & .85 & .34 & 2.10 & .34 & .18 & .02 & 98.83 \\
\hline GO-5 & $\mathrm{HU}$ & hc2 & 1 & W-214718 & c & RBM & 47.50 & 13.80 & 5.50 & 7.04 & 10.00 & 10.40 & 2.50 & 1.03 & .41 & 2.20 & .32 & .19 & .02 & 100.91 \\
\hline GO-6 & HU & hc1o & 1 & W-214735 & c & RBM & 45.80 & 9.70 & 3.20 & 8.90 & 16.00 & 10.30 & 1.70 & .70 & .24 & 1.60 & .21 & .19 & .02 & 98.56 \\
\hline GO-7 & HU & hc1o & 1 & W-214717 & c & RBM & 46.60 & 12.50 & 4.20 & 7.76 & 10.70 & 11.30 & 2.20 & 1.42 & .42 & 2.20 & .35 & .18 & .02 & 99.85 \\
\hline $\mathrm{GO}-8$ & $\mathrm{HU}$ & hc1y & 1 & W-227873 & c & $\mathrm{RBM}+\mathrm{DAC}$ & 46.50 & 15.40 & 3.60 & 8.30 & 7.30 & 10.40 & 4.30 & .82 & .49 & 2.50 & .44 & .15 & .07 & 100.27 \\
\hline
\end{tabular}


Table 1.-Chemical analyses-Continued

\begin{tabular}{|c|c|c|c|c|c|c|c|c|c|c|c|c|c|c|c|c|c|c|c|c|}
\hline $\begin{array}{c}\text { Sample } \\
\text { locality } \\
\text { No. }\end{array}$ & $\begin{array}{l}\text { Vol- } \\
\text { cano }\end{array}$ & Unit & $\begin{array}{c}\text { Map } \\
\text { sheet } \\
\text { No. }\end{array}$ & $\begin{array}{l}\text { Laboratory } \\
\text { No. }\end{array}$ & Remark & Collector & $\mathrm{SiO}_{2}$ & $\mathrm{Al}_{2} \mathrm{O}_{3}$ & $\mathrm{Fe}_{2} \mathrm{O}_{3}$ & $\mathrm{FeO}$ & $\mathrm{MgO}$ & $\mathrm{CaO}$ & $\mathrm{Na}_{2} \mathrm{O}$ & $\mathrm{K}_{2} \mathrm{O}$ & $\mathrm{H}_{2} \mathrm{O}$ & $\mathrm{TiO}_{2}$ & $\mathrm{P}_{2} \mathrm{O}_{5}$ & $\mathrm{MnO}$ & $\mathrm{CO}_{2}$ & Total \\
\hline GO-9 & $\mathrm{HU}$ & hc & $\overline{1}$ & W-217453 & c & RBM & 46.00 & 14.00 & 11.10 & 1.70 & 9.00 & 10.50 & 2.40 & .96 & .52 & 2.20 & .31 & .20 & .02 & 98.91 \\
\hline GO-10 & HU & he & 1 & W-217454 & c & RBM & 45.50 & 13.80 & 7.50 & 4.80 & 9.10 & 10.90 & 2.00 & .78 & 1.43 & 2.10 & .30 & .23 & .02 & 98.46 \\
\hline GO-11 & $\mathrm{HU}$ & h3 & 1 & W-227870 & c & RBM & 47.10 & 14.00 & 2.90 & 10.00 & 10.30 & 10.20 & 2.60 & .59 & .30 & 2.00 & .28 & .18 & .08 & 100.53 \\
\hline GO-12 & HU & h1y & 1 & W-227869 & c & RBM & 46.30 & 14.30 & 1.90 & 11.00 & 9.60 & 10.10 & 2.60 & .70 & .34 & 2.10 & .32 & .19 & .07 & 99.52 \\
\hline GO-13 & $\mathrm{HU}$ & hc2 & 1 & W-214716 & c & RBM & 46.80 & 13.90 & 8.90 & 3.80 & 9.10 & 10.40 & 2.20 & .77 & .47 & 2.10 & .33 & .17 & .02 & 98.96 \\
\hline GO-14 & $\mathrm{HU}$ & h1y & 1 & W-227847 & c & RBM & 47.00 & 14.50 & 1.90 & 10.90 & 10.00 & 10.70 & 2.70 & .70 & .41 & 2.00 & .33 & .19 & .01 & 101.34 \\
\hline GO-15 & $\mathrm{HU}$ & hc10 & 1 & W-214726 & c & RBM & 47.10 & 15.50 & 5.60 & 6.10 & 7.10 & 11.90 & 2.70 & .85 & .35 & 2.40 & .32 & .14 & .02 & 100.08 \\
\hline GO-16 & HU & h1o & 1 & W-217456 & c & RBM & 47.20 & 15.90 & 4.70 & 7.50 & 6.30 & 10.80 & 2.80 & .86 & .47 & 2.50 & .38 & .21 & .01 & 99.63 \\
\hline GO-18 & $\mathrm{HU}$ & hc3 & 1 & W-217482 & c & RBM & 46.60 & 14.90 & 3.00 & 9.60 & 8.70 & 10.20 & 2.80 & .85 & .47 & 2.20 & .31 & .23 & .02 & 99.88 \\
\hline GO-19 & $\mathrm{HU}$ & h3 & 1 & W-217497 & c & RBM & 46.80 & 14.40 & 2.50 & 10.00 & 8.90 & 10.10 & 2.70 & .78 & .43 & 2.10 & .30 & .20 & .02 & 99.23 \\
\hline GO-21 & ML & $k 2$ & 1 & W-227860 & c & RBM & 51.00 & 12.30 & 2.00 & 9.30 & 11.50 & 9.70 & 2.00 & .22 & .43 & 2.00 & .25 & .18 & .02 & 100.90 \\
\hline GO-22 & $\mathrm{HU}$ & he1y & 1 & W-214743 & c & RBM & 47.30 & 14.40 & 3.70 & 7.90 & 8.50 & 11.00 & 2.60 & .73 & .28 & 2.00 & .29 & .17 & .02 & 98.89 \\
\hline GO-23 & ML & $k 2$ & 1 & W-227861 & c & RBM & 51.90 & 13.40 & 1.80 & 9.60 & 8.40 & 10.20 & 2.10 & .26 & .31 & 2.00 & .27 & .17 & .06 & 100.47 \\
\hline GO-24 & ML & k2 & 1 & W-227848 & c & RBM & 51.30 & 13.60 & 1.90 & 8.90 & 8.00 & 10.30 & 2.30 & .29 & .67 & 2.10 & .28 & .16 & .01 & 99.81 \\
\hline GO-25 & $\mathrm{HU}$ & hc & 1 & W-214715 & c & RBM & 46.20 & 13.30 & 3.00 & 9.16 & 10.00 & 10.50 & 2.30 & 1.07 & .78 & 2.40 & .32 & .19 & .02 & 99.24 \\
\hline GO-27 & $\mathrm{HU}$ & h1y & 1 & W-214725 & c & RBM & 47.00 & 13.60 & 2.90 & 8.90 & 10.50 & 12.00 & 2.10 & 1.21 & .37 & 1.60 & .21 & .16 & .02 & 100.57 \\
\hline GO-29 & ML & $k 2$ & 1 & W-227859 & c & RBM & 49.20 & 10.80 & 2.20 & 9.10 & 15.50 & 8.30 & 1.60 & .18 & .54 & 1.70 & .22 & .17 & .03 & 99.54 \\
\hline GO-30 & HU & hc1y & 1 & W-214719 & c & RBM & 46.50 & 13.40 & 8.10 & 4.76 & 10.00 & 9.80 & 2.40 & 1.14 & .56 & 2.20 & .31 & .20 & .02 & 99.39 \\
\hline GO-31 & ML & 12 & 1 & W-227862 & $c$ & RBM & 51.70 & 13.70 & 2.10 & 8.50 & 8.10 & 10.60 & 2.10 & .24 & .34 & 2.10 & .25 & .16 & .02 & 99.91 \\
\hline GO-32 & $\mathrm{HU}$ & hc10 & 1 & W-214720 & c & RBM & 46.70 & 14.10 & 7.10 & 5.72 & 8.70 & 9.80 & 2.50 & .94 & .58 & 2.10 & .31 & .21 & .02 & 98.78 \\
\hline GO-33 & $\mathrm{HU}$ & hc & 1 & W-217459 & c & RBM & 46.30 & 15.50 & 8.80 & 4.90 & 6.00 & 10.00 & 2.90 & .86 & .79 & 2.70 & .37 & .20 & .01 & 99.33 \\
\hline GO-34 & HU & h3 & 1 & W-227851 & c & RBM & 46.30 & 13.90 & 2.80 & 9.20 & 10.20 & 11.20 & 2.50 & .53 & .40 & 1.90 & .30 & .18 & .01 & 99.42 \\
\hline GO-35 & $\mathrm{HU}$ & h1y & 1 & W-227923 & c & $\mathrm{RBM}+\mathrm{DAC}$ & 47.10 & 15.40 & 2.40 & 9.60 & 7.10 & 11.30 & 2.80 & .93 & .34 & 2.30 & .43 & .18 & .04 & 99.92 \\
\hline GO-36 & HU & h10 & 1 & W-217492 & c & RBM & 45.10 & 11.50 & 4.70 & 8.10 & 16.20 & 8.70 & 2.20 & .54 & .36 & 1.60 & .22 & .19 & .02 & 99.43 \\
\hline GO-37 & $\mathrm{HU}$ & ho3 & 1 & W-214745 & c & RBM & 45.90 & 13.90 & 4.60 & 8.10 & 9.70 & 9.80 & 2.40 & 1.21 & .41 & 2.30 & .33 & .18 & .02 & 98.85 \\
\hline GO-38 & $\mathrm{HU}$ & hc10 & 1 & W-214744 & c & RBM & 47.00 & 12.80 & 2.20 & 8.90 & 12.30 & 10.50 & 2.20 & .60 & .33 & 1.70 & .22 & .18 & .02 & 98.95 \\
\hline GO-39 & HU & h1o & 1 & W-227924 & c & $\mathrm{RBM}+\mathrm{DAC}$ & 46.70 & 13.00 & 2.90 & 8.70 & 11.10 & 11.40 & 2.10 & .72 & .25 & 1.70 & .30 & .17 & .02 & 99.06 \\
\hline GO-40 & HU & h1y & 1 & W-217464 & c & $\mathrm{RBM}$ & 46.70 & 14.80 & 4.00 & 7.80 & 8.40 & 11.90 & 2.20 & .51 & .45 & 2.00 & .23 & .18 & .01 & 99.18 \\
\hline GO-41 & ML & $k 2$ & 1 & W-227854 & c & RBM & 51.50 & 13.60 & 9.20 & 2.70 & 7.60 & 10.80 & 2.30 & .29 & .47 & 2.10 & .28 & .17 & .01 & 101.02 \\
\hline GO-42 & $\mathrm{HU}$ & hc1y & 1 & W-217463 & c & RBM & 46.50 & 14.60 & 9.20 & 3.00 & 8.60 & 11.60 & 2.10 & .47 & .57 & 1.90 & .22 & .18 & .02 & 98.96 \\
\hline GO-43 & HU & h1o & 1 & W-217493 & c & RBM & 47.00 & 14.70 & 3.40 & 8.10 & 9.30 & 10.70 & 2.60 & .76 & .40 & 2.00 & .30 & .22 & .02 & 99.50 \\
\hline GO-44 & HU & he & 1 & W-217494 & c & RBM & 46.20 & 12.90 & 9.10 & 3.20 & 12.40 & 10.10 & 1.70 & .55 & 1.16 & 1.80 & .26 & .18 & .02 & 99.57 \\
\hline GO-45 & $\mathrm{HU}$ & hc10 & 1 & W-214742 & c & RBM & 45.90 & 14.10 & 5.20 & 6.80 & 9.80 & 10.80 & 2.40 & .93 & .72 & 2.20 & .34 & .17 & .02 & 99.38 \\
\hline GO-46 & HU & h1y & 1 & W-214727 & c & RBM & 48.00 & 14.70 & 4.10 & 7.70 & 7.80 & 12.60 & 2.30 & .53 & .42 & 2.00 & .19 & .16 & .02 & 100.52 \\
\hline GO-47 & HU & hc10 & 1 & W-214746 & c & RBM & 46.30 & 14.10 & 9.30 & 2.60 & 9.50 & 10.40 & 2.50 & .85 & .76 & 2.00 & .30 & .16 & .02 & 98.79 \\
\hline GO-48 & HU & hc1o & 1 & W-217466 & c & RBM & 45.90 & 11.90 & 5.10 & 6.60 & 14.00 & 11.30 & 1.90 & .62 & .25 & 1.70 & .24 & .17 & .01 & 99.69 \\
\hline GO-49 & $\mathrm{HU}$ & hc2 & 1 & W-227930 & c & RBM & 47.80 & 13.20 & 3.80 & 7.70 & 12.10 & 11.00 & 1.70 & .37 & .59 & 1.40 & .21 & .17 & .02 & 100.06 \\
\hline GO-50 & HU & hc10 & 1 & W-217468 & c & RBM & 47.40 & 14.90 & 3.50 & 8.50 & 8.20 & 10.70 & 2.40 & .64 & .44 & 1.90 & .29 & .19 & .02 & 99.08 \\
\hline GO-51 & HU & hc1o & 1 & W-217485 & c & RBM & 46.60 & 14.80 & 2.80 & 9.10 & 8.70 & 10.50 & 2.40 & .80 & .55 & 2.20 & .30 & .17 & .02 & 98.94 \\
\hline GO-53 & $\mathrm{HU}$ & hc2 & 1 & W-217480 & c & RBM & 47.80 & 16.00 & 6.10 & 6.20 & 5.90 & 8.30 & 3.70 & 1.40 & .41 & 2.90 & .50 & .17 & .01 & 99.39 \\
\hline
\end{tabular}


Table 1.-Chemical analyses-Continued

\begin{tabular}{|c|c|c|c|c|c|c|c|c|c|c|c|c|c|c|c|c|c|c|c|c|}
\hline $\begin{array}{c}\text { Sample } \\
\text { locality } \\
\text { No. }\end{array}$ & $\begin{array}{l}\text { Vol- } \\
\text { cano }\end{array}$ & Unit & $\begin{array}{c}\text { Map } \\
\text { sheet } \\
\text { No. }\end{array}$ & $\begin{array}{l}\text { Laboratory } \\
\text { No. }\end{array}$ & Remark & Collector & $\mathrm{SiO}_{2}$ & $\mathrm{Al}_{2} \mathrm{O}_{3}$ & $\mathrm{Fe}_{2} \mathrm{O}_{3}$ & $\mathrm{FeO}$ & $\mathrm{MgO}$ & $\mathrm{CaO}$ & $\mathrm{Na}_{2} \mathrm{O}$ & $\mathrm{K}_{2} \mathrm{O}$ & $\mathrm{H}_{2} \mathrm{O}$ & $\mathrm{TiO}_{2}$ & $\mathrm{P}_{2} \mathrm{O}_{5}$ & $\mathrm{MnO}$ & $\mathrm{CO}_{2}$ & Total \\
\hline GO-54 & $\mathrm{ML}$ & k5-1859 & 1 & W-227850 & c & RBM & 50.70 & 12.50 & 1.70 & 8.80 & 12.20 & 9.80 & 2.10 & .22 & .43 & 1.80 & .25 & .16 & .02 & 100.68 \\
\hline GO-55 & $\mathrm{HU}$ & hc2 & 1 & W-217489 & c & RBM & 46.40 & 13.90 & 5.30 & 7.00 & 10.50 & 10.50 & 2.40 & .65 & .60 & 1.90 & .30 & .20 & .02 & 99.67 \\
\hline GO-56 & HU & hc2 & 1 & W-217488 & c & RBM & 46.40 & 14.50 & 3.10 & 9.10 & 9.10 & 10.50 & 2.70 & .83 & .44 & 2.30 & .30 & .18 & .02 & 99.47 \\
\hline GO-57 & $\mathrm{HU}$ & he & 1 & W-214741 & c & RBM & 47.60 & 14.80 & 6.60 & 6.60 & 5.50 & 9.90 & 3.10 & 1.70 & .73 & 3.00 & .38 & .19 & .02 & 100.12 \\
\hline GO-61 & $\mathrm{HU}$ & hc2 & 1 & W-217478 & c & RBM & 45.90 & 14.10 & 6.30 & 6.90 & 10.60 & 9.50 & 2.50 & .80 & .67 & 2.10 & .32 & .16 & .01 & 99.86 \\
\hline GO-62 & $\mathrm{HU}$ & hc2 & 1 & W-214724 & c & RBM & 47.50 & 14.60 & 5.10 & 7.52 & 8.40 & 10.30 & 2.80 & 1.04 & .30 & 2.40 & .32 & .21 & .02 & 100.51 \\
\hline GO-65 & $\mathrm{HU}$ & hc & 1 & W-217513 & c & $\mathrm{RBM}+\mathrm{DAC}$ & 46.00 & 12.10 & 2.50 & 9.40 & 12.50 & 11.20 & 2.10 & .87 & .62 & 2.10 & .27 & .14 & .02 & 99.82 \\
\hline GO-67 & $\mathrm{HU}$ & hc2 & 1 & W-214693 & c & RBM & 47.70 & 13.30 & 3.90 & 8.56 & 10.00 & 11.00 & 2.50 & .74 & .36 & 2.00 & .31 & .20 & .02 & 100.59 \\
\hline GO-68 & $\mathrm{HU}$ & hc & 1 & W-214733 & c & RBM & 46.10 & 12.80 & 4.80 & 7.40 & 12.40 & 10.00 & 2.20 & .62 & .81 & 1.80 & .26 & .16 & .02 & 99.37 \\
\hline GO-70 & $\mathrm{HU}$ & hc1o & 1 & W-217476 & c & RBM & 46.10 & 14.00 & 3.10 & 9.40 & 9.30 & 10.10 & 2.50 & .95 & .41 & 2.40 & .31 & .18 & .02 & 98.77 \\
\hline GO-71 & $\mathrm{HU}$ & h1o & 1 & W-214721 & c & RBM & 47.00 & 14.30 & 6.10 & 6.32 & 8.70 & 10.30 & 2.30 & 1.18 & .47 & 2.40 & .32 & .19 & .02 & 99.60 \\
\hline GO-72 & $\mathrm{HU}$ & hc & 1 & W-214737 & c & RBM & 46.90 & 15.00 & 3.60 & 9.00 & 7.00 & 11.70 & 2.50 & .80 & .43 & 2.40 & .29 & .17 & .02 & 99.81 \\
\hline GO-73 & $\mathrm{HU}$ & he & 1 & W-214698 & c & RBM & 49.00 & 14.80 & 2.90 & 8.08 & 8.70 & 11.60 & 2.30 & .58 & .33 & 1.80 & .25 & .19 & .02 & 100.55 \\
\hline GO-74 & $\mathrm{HU}$ & hc1o & 1 & W-214736 & c & RBM & 46.90 & 15.10 & 4.70 & 8.10 & 6.20 & 9.80 & 3.00 & 1.06 & .62 & 2.60 & .33 & .17 & .02 & 98.60 \\
\hline GO-75 & $\mathrm{HU}$ & hc10 & 1 & W-217475 & c & RBM & 47.10 & 13.10 & 4.00 & 7.50 & 12.50 & 10.20 & 2.00 & .51 & .37 & 1.60 & .23 & .17 & .02 & 99.30 \\
\hline GO-76 & HU & hc & 1 & W-214700 & c & RBM & 46.80 & 12.80 & 6.30 & 5.28 & 11.80 & 10.30 & 2.10 & .82 & .73 & 2.10 & .30 & .20 & .02 & 99.55 \\
\hline GO-77 & $\mathrm{HU}$ & hc1o & 1 & W-217484 & c & RBM & 47.00 & 15.70 & 5.70 & 6.90 & 5.80 & 10.70 & 3.10 & .97 & .53 & 2.50 & .37 & .21 & .06 & 99.54 \\
\hline GO-80 & $\mathrm{HU}$ & hc1y & 1 & W-217469 & c & RBM & 46.80 & 15.60 & 11.50 & 2.40 & 5.30 & 9.00 & 3.50 & 1.30 & .65 & 3.00 & .45 & .19 & .01 & 99.70 \\
\hline GO-81 & $\mathrm{HU}$ & hc10 & 1 & W-214707 & c & RBM & 47.40 & 12.70 & 3.70 & 7.76 & 11.80 & 11.20 & 2.20 & .67 & .36 & 1.80 & .23 & .19 & .02 & 100.03 \\
\hline GO-82 & $\mathrm{HU}$ & hc & 1 & W-214696 & c & RBM & 47.50 & 14.30 & 3.10 & 9.12 & 8.20 & 11.30 & 2.50 & .76 & .32 & 2.30 & .29 & .19 & .02 & 99.90 \\
\hline GO-83 & $\mathrm{HU}$ & h & 1 & W-214699 & c & RBM & 50.10 & 16.90 & 5.60 & 4.52 & 6.10 & 11.80 & 2.50 & .76 & .66 & 1.80 & .25 & .14 & .02 & 101.15 \\
\hline GO-84 & HU & hc10 & 1 & W-214706 & c & RBM & 48.30 & 15.50 & 9.30 & 3.88 & 5.70 & 10.20 & 2.70 & 1.19 & .29 & 2.80 & .42 & .21 & .02 & 100.51 \\
\hline GO-85 & $\mathrm{HU}$ & hc1o & 1 & W-214747 & c & RBM & 45.50 & 12.00 & 3.10 & 9.60 & 13.70 & 10.10 & 2.10 & .71 & .34 & 1.70 & .25 & .18 & .02 & 99.30 \\
\hline GO-86 & HU & h1y & 1 & W-214706 & c & RBM & 46.60 & 12.50 & 5.30 & 6.48 & 12.40 & 9.80 & 2.00 & .84 & .66 & 2.10 & .29 & .18 & .02 & 99.17 \\
\hline GO-90 & $\mathrm{HU}$ & hc10 & 1 & W-214748 & c & RBM & 46.20 & 15.40 & 8.40 & 4.00 & 6.60 & 9.90 & 3.30 & 1.25 & .61 & 2.50 & .42 & .18 & .02 & 98.78 \\
\hline GO-91 & $\mathrm{HU}$ & hc1y & 1 & W-217474 & c & RBM & 45.60 & 14.10 & 2.90 & 9.60 & 9.70 & 9.40 & 2.50 & .84 & .89 & 2.50 & .32 & .16 & .01 & 98.52 \\
\hline GO-93 & $\mathrm{HU}$ & hc1o & 1 & W-227929 & c & $\mathrm{RBM}+\mathrm{DAC}$ & 46.90 & 15.20 & 3.50 & 9.40 & 6.70 & 11.60 & 2.50 & .90 & .46 & 2.60 & .41 & .18 & .02 & 100.37 \\
\hline GO-94 & $\mathrm{HU}$ & hc1y & 1 & W-217471 & c & $\mathrm{RBM}$ & 47.40 & 15.10 & 9.10 & 4.20 & 5.90 & 9.10 & 3.40 & 1.30 & .64 & 2.90 & .43 & .19 & .01 & 99.67 \\
\hline GO-96 & $\mathrm{HU}$ & hc2 & 1 & W-214704 & c & RBM & 46.90 & 14.80 & 7.90 & 5.24 & 6.70 & 10.30 & 3.30 & 1.17 & .56 & 2.50 & .37 & .18 & .02 & 99.94 \\
\hline GO-97 & $\mathrm{HU}$ & hc1o & 1 & W-214703 & c & RBM & 46.60 & 12.20 & 2.50 & 9.60 & 13.80 & 9.70 & 2.20 & .71 & .27 & 1.70 & .25 & .18 & .02 & 99.73 \\
\hline GO-98 & $\mathrm{HU}$ & h2 & 1 & W-217567 & c & RBM & 46.30 & 13.90 & 5.70 & 6.80 & 11.30 & 10.00 & 2.40 & .69 & .83 & 2.00 & .27 & .16 & .02 & 100.37 \\
\hline GO-101 & $\mathrm{HU}$ & hc2 & 1 & W-214729 & c & RBM & 47.30 & 14.60 & 3.50 & 8.80 & 9.30 & 10.00 & 2.70 & .78 & .44 & 2.00 & .28 & .16 & .02 & 99.88 \\
\hline GO-102 & $\mathrm{HU}$ & h3 & 1 & W-214750 & c & RBM & 47.30 & 16.20 & 1.80 & 10.30 & 6.10 & 8.30 & 3.60 & 1.37 & .35 & 2.60 & .47 & .19 & .02 & 98.60 \\
\hline GO-105 & $\mathrm{HU}$ & hc1o & 1 & W-217498 & c & RBM & 46.90 & 15.00 & 5.50 & 6.40 & 8.60 & 11.10 & 2.40 & .67 & .73 & 1.90 & .29 & .18 & .01 & 99.68 \\
\hline GO-106 & $\mathrm{HU}$ & h1y & 1 & W-217502 & c & $\mathrm{RBM}+\mathrm{DAC}$ & 47.00 & 14.60 & 4.90 & 9.70 & 5.60 & 9.70 & 3.40 & 1.30 & .50 & 3.00 & .45 & .21 & .01 & 100.37 \\
\hline GO-107 & $\mathrm{HU}$ & hc1y & 1 & W-217503 & c & $\mathrm{RBM}+\mathrm{DAC}$ & 47.10 & 14.10 & 4.80 & 8.40 & 7.80 & 9.90 & 3.10 & 1.10 & .45 & 2.60 & .38 & .19 & .02 & 99.94 \\
\hline GO-108 & $\mathrm{HU}$ & h1o & 1 & W-217452 & c & RBM & 46.30 & 14.40 & 4.10 & 8.60 & 9.20 & 10.50 & 2.60 & .92 & .27 & 2.50 & .33 & .15 & .01 & 99.88 \\
\hline GO-109 & $\mathrm{HU}$ & hc2 & 1 & W-217504 & c & $\mathrm{RBM}+\mathrm{DAC}$ & 46.80 & 13.40 & 4.40 & 8.60 & 9.00 & 10.20 & 2.80 & .93 & .52 & 2.40 & .35 & .20 & .01 & 99.61 \\
\hline GO-110 & $\mathrm{HU}$ & hc2 & 1 & W-217505 & c & $\mathrm{RBM}+\mathrm{DAC}$ & 46.70 & 13.40 & 4.30 & 8.60 & 7.40 & 9.50 & 4.00 & 1.10 & .45 & 2.50 & .37 & .18 & .01 & 98.51 \\
\hline GO-111 & ML & $\mathrm{k} 2$ & 1 & W-227904 & c & $\mathrm{RBM}$ & 50.30 & 11.10 & 1.30 & 9.50 & 15.90 & 8.60 & 1.80 & .26 & .44 & 1.70 & .20 & .14 & .03 & 101.27 \\
\hline
\end{tabular}


Table 1.-Chemical analyses-Continued

\begin{tabular}{|c|c|c|c|c|c|c|c|c|c|c|c|c|c|c|c|c|c|c|c|c|}
\hline $\begin{array}{c}\text { Sample } \\
\text { locality } \\
\text { No. }\end{array}$ & $\begin{array}{l}\text { Vol- } \\
\text { cano }\end{array}$ & Unit & $\begin{array}{l}\text { Map } \\
\text { sheet } \\
\text { No. }\end{array}$ & $\begin{array}{l}\text { Laboratory } \\
\text { No. }\end{array}$ & Remark & Collector & $\mathrm{SiO}_{2}$ & $\mathrm{Al}_{2} \mathrm{O}_{3}$ & $\mathrm{Fe}_{2} \mathrm{O}_{3}$ & $\mathrm{FeO}$ & $\mathrm{MgO}$ & $\mathrm{CaO}$ & $\mathrm{Na}_{2} \mathrm{O}$ & $\mathrm{K}_{2} \mathrm{O}$ & $\mathrm{H}_{2} \mathrm{O}$ & $\mathrm{TiO}_{2}$ & $\mathrm{P}_{2} \mathrm{O}_{5}$ & $\mathrm{MnO}$ & $\mathrm{CO}_{2}$ & Total \\
\hline GO-112 & ML & K2 & 1 & W-227905 & c & $\mathrm{RBM}$ & 51.50 & 13.80 & 1.70 & 8.80 & 7.40 & 10.60 & 2.20 & .30 & .52 & 2.20 & .28 & .16 & .03 & 99.49 \\
\hline GO-113 & ML & $k 2$ & 1 & W-227906 & c & $\mathrm{RBM}$ & 49.30 & 11.60 & 2.10 & 8.80 & 13.60 & 9.40 & 1.90 & .27 & .31 & 1.90 & .22 & .18 & .04 & 99.62 \\
\hline GO-114 & $\mathrm{HU}$ & hc & 1 & W-217451 & c & $\mathrm{RBM}$ & 45.40 & 13.00 & 12.20 & .08 & 11.40 & 11.10 & 2.00 & .50 & 1.49 & 1.60 & .15 & .17 & .01 & 99.10 \\
\hline GO-115 & $\mathrm{HU}$ & holy & 1 & W-227946 & c & $\mathrm{RBM}+\mathrm{DAC}$ & 47.80 & 13.20 & 3.50 & 8.60 & 11.80 & 10.80 & 2.10 & .53 & .20 & 1.70 & .26 & .18 & .00 & 100.67 \\
\hline GO-116 & $\mathrm{HU}$ & hc2 & 1 & W-227932 & c & $\mathrm{RBM}+\mathrm{DAC}$ & 46.70 & 14.50 & 5.80 & 7.20 & 8.70 & 10.20 & 2.70 & .96 & .28 & 2.30 & .41 & .19 & .03 & 99.97 \\
\hline GP-1 & ML & $\mathrm{K} 3$ & 1 & D-276860 & $b$ & EWW & 51.80 & 13.40 & 12.30 & nd & 7.28 & 10.50 & 2.18 & .44 & .02 & 2.27 & .29 & .17 & nd & 100.65 \\
\hline GP-2 & ML & $k 2$ & 1 & D-276859 & b & EWW & 48.50 & 9.12 & 13.10 & nd & 19.60 & 7.40 & 1.55 & .24 & .00 & 1.54 & .16 & .17 & nd & 101.38 \\
\hline GP-3 & $\mathrm{ML}$ & $k 2$ & 1 & D-276098 & $\mathrm{b}$ & EWW & 51.70 & 13.60 & 12.40 & nd & 7.78 & 10.50 & 2.16 & .35 & .00 & 2.08 & .25 & .17 & nd & 100.99 \\
\hline GP-4 & ML & $k 3$ & 1 & D-276099 & $\mathrm{b}$ & EWW & 51.30 & 12.90 & 11.20 & nd & 9.74 & 10.40 & 2.11 & .38 & .00 & 2.32 & .26 & .16 & nd & 100.77 \\
\hline GP-5 & ML & $k 3$ & 1 & D-276095 & $\mathrm{b}$ & EWW & 51.80 & 13.80 & 12.20 & nd & 6.77 & 10.70 & 2.38 & .48 & .00 & 2.46 & .30 & .17 & nd & 101.06 \\
\hline GP-6 & ML & k2 & 1 & D-276861 & $b$ & EWW & 51.60 & 13.00 & 12.00 & nd & 8.92 & 10.60 & 1.95 & .31 & .06 & 2.19 & .22 & .17 & nd & 101.02 \\
\hline GP-7 & ML & k1y & 1 & D-276862 & b & EWW & 50.30 & 13.10 & 12.20 & nd & 8.99 & 9.95 & 1.82 & .32 & 1.35 & 2.17 & .25 & .17 & nd & 100.62 \\
\hline GP-8 & ML. & kc2 & 1 & D-276097 & $\mathrm{b}$ & EWW & 50.90 & 12.90 & 12.10 & nd & 9.15 & 10.30 & 2.10 & .39 & .00 & 2.07 & .25 & .17 & nd & 100.33 \\
\hline GP-9 & ML & $k 2$ & 1 & D-276857 & $\mathrm{b}$ & EWW & 51.50 & 13.60 & 12.10 & nd & 7.73 & 10.90 & 2.07 & .34 & .00 & 2.16 & .22 & .17 & nd & 100.79 \\
\hline GP-10 & ML & $k 2$ & 1 & D-276096 & $\mathrm{b}$ & EWW & 51.80 & 13.80 & 12.40 & nd & 7.02 & 10.70 & 2.23 & .38 & .00 & 2.15 & .27 & .17 & nd & 100.92 \\
\hline GP-11 & ML & k2 & 1 & D-276094 & $\mathrm{b}$ & EWW & 51.80 & 13.80 & 12.30 & nd & 7.01 & 10.80 & 2.27 & .39 & .00 & 2.14 & .26 & .17 & nd & 100.94 \\
\hline GP-12 & ML & k1y & 1 & D-276856 & $\mathrm{b}$ & EWW & 51.80 & 13.60 & 11.90 & nd & 7.08 & 10.80 & 2.13 & .32 & .03 & 2.21 & .23 & .16 & nd & 100.26 \\
\hline GP-13 & ML & $k 3$ & 1 & D-276091 & $\mathrm{b}$ & EWW & 51.80 & 13.60 & 12.10 & nd & 7.37 & 10.80 & 2.13 & .32 & .00 & 2.28 & .23 & .17 & nd & 100.80 \\
\hline GP-14 & ML & $k 2$ & 1 & D-276092 & $b$ & EWW & 49.60 & 10.80 & 12.30 & nd & 15.50 & 8.63 & 1.72 & .27 & .00 & 1.81 & .19 & .17 & nd & 100.99 \\
\hline GP-15 & ML. & k2 & 1 & D-276093 & $b$ & EWW & 51.80 & 13.80 & 12.30 & nd & 7.22 & 10.70 & 2.32 & .40 & .00 & 2.18 & .27 & .17 & nd & 101.16 \\
\hline GP-16 & ML & k5-1859 & 1 & D-276858 & $\mathrm{b}$ & EWW & 50.70 & 12.80 & 12.50 & nd & 9.60 & 10.30 & 2.09 & .37 & .04 & 2.04 & .23 & .17 & nd & 100.84 \\
\hline GP-17 & ML & k2 & 1 & D-276086 & $\mathrm{b}$ & EWW & 51.70 & 13.50 & 12.30 & nd & 7.69 & 10.40 & 2.25 & .37 & .00 & 2.05 & .24 & .17 & nd & 100.67 \\
\hline GP-18 & ML & $k 2$ & 1 & D-276087 & $\mathrm{b}$ & EWW & 51.70 & 13.70 & 12.30 & nd & 7.07 & 10.80 & 2.27 & .39 & .00 & 2.14 & .26 & .17 & nd & 100.80 \\
\hline GP-19 & ML & k2 & 1 & D-276090 & $\mathrm{b}$ & EWW & 51.50 & 13.50 & 12.40 & nd & 7.11 & 10.80 & 2.24 & .40 & .00 & 2.41 & .26 & .17 & nd & 100.79 \\
\hline GP-20 & ML & $k 2$ & 1 & D-276852 & $\mathrm{b}$ & EWW & 51.30 & 13.40 & 12.40 & nd & 7.35 & 10.70 & 2.15 & .40 & .00 & 2.38 & .26 & .17 & nd & 100.51 \\
\hline GP-21 & ML & $k 3$ & 1 & D-276085 & $\mathrm{b}$ & EWW & 47.90 & 9.16 & 13.00 & nd & 19.90 & 7.46 & 1.52 & .26 & .00 & 1.62 & .18 & .17 & nd & 101.17 \\
\hline GP-22 & ML & $k 2$ & 1 & D-276084 & $\mathrm{b}$ & EWW & 49.70 & 11.00 & 12.30 & nd & 15.10 & 8.65 & 1.88 & .28 & .00 & 1.82 & .20 & .16 & nd & 101.09 \\
\hline GP-23 & ML & k1y & 1 & D-276081 & b & EWW & 51.00 & 13.00 & 12.30 & nd & 9.43 & 10.20 & 2.17 & .40 & .00 & 2.10 & .26 & .17 & nd & 101.03 \\
\hline GP-24 & ML & $k 2$ & 1 & D-276083 & $\mathrm{b}$ & EWW & 51.40 & 13.50 & 12.30 & nd & 7.84 & 10.40 & 2.29 & .37 & .21 & 2.05 & .25 & .17 & nd & 100.78 \\
\hline GP-25 & ML & k1y & 1 & D-276089 & b & EWW & 51.60 & 14.10 & 12.00 & nd & 7.01 & 11.10 & 2.31 & .39 & .00 & 2.11 & .25 & .17 & nd & 101.04 \\
\hline GP-26 & ML & k1y & 1 & D-276082 & b & EWW & 50.90 & 12.60 & 12.00 & nd & 9.97 & 10.00 & 2.06 & .32 & .35 & 2.03 & .22 & .17 & nd & 100.62 \\
\hline GP-27 & ML & $k 3$ & 1 & D-276088 & $b$ & EWW & 50.90 & 12.40 & 11.40 & nd & 11.00 & 10.10 & 2.04 & .37 & .00 & 2.22 & .23 & .16 & nd & 100.82 \\
\hline GP-28 & ML & k1y & 1 & D-278126 & $\mathrm{b}$ & $C D C$ & 49.70 & 11.40 & 12.40 & nd & 14.40 & 9.02 & 1.73 & .33 & .06 & 1.78 & .22 & .17 & nd & 101.21 \\
\hline GP-29 & ML & $k 2$ & 1 & D-276080 & $\mathrm{b}$ & EWW & 51.60 & 13.70 & 12.30 & nd & 7.23 & 10.70 & 2.24 & .39 & .00 & 2.11 & .25 & .17 & nd & 100.69 \\
\hline GP-30 & ML & 12 & 1 & D-276079 & b & EWW & 51.80 & 13.80 & 12.40 & nd & 7.20 & 10.80 & 2.22 & .39 & .00 & 2.15 & .26 & .17 & nd & 101.19 \\
\hline GP-31 & ML & k1y & 1 & D-278127 & b & $\mathrm{CDC}$ & 52.30 & 13.70 & 11.60 & nd & 7.69 & 10.80 & 2.19 & .35 & .05 & 2.21 & .24 & .16 & nd & 101.29 \\
\hline GP-32 & ML & k1y & 1 & D-278128 & $b$ & $C D C$ & 51.00 & 13.00 & 12.20 & nd & 9.25 & 10.20 & 2.10 & .39 & .37 & 2.09 & .24 & .17 & nd & 101.01 \\
\hline GP-33 & ML & $k 2$ & 1 & D-276078 & b & EWW & 51.70 & 13.80 & 12.30 & nd & 7.08 & 10.80 & 2.19 & .38 & .00 & 2.14 & .26 & .17 & nd & 100.82 \\
\hline GP-34 & ML & $k 3$ & 1 & D-276076 & b & EWW & 49.30 & 10.60 & 12.90 & nd & 15.50 & 8.65 & 1.65 & .29 & .00 & 1.84 & .20 & .17 & nd & 101.10 \\
\hline GP-35 & ML & K3 & 1 & D-276077 & b & EWW & 51.60 & 13.60 & 12.40 & nd & 7.18 & 10.60 & 2.22 & .40 & .02 & 2.20 & .27 & .17 & nd & 100.66 \\
\hline
\end{tabular}


Table 1.-Chemical analyses-Continued

\begin{tabular}{|c|c|c|c|c|c|c|c|c|c|c|c|c|c|c|c|c|c|c|c|c|}
\hline $\begin{array}{c}\text { Sample } \\
\text { locality } \\
\text { No. }\end{array}$ & $\begin{array}{l}\text { Vol- } \\
\text { cano }\end{array}$ & Unit & $\begin{array}{c}\text { Map } \\
\text { sheet } \\
\text { No. }\end{array}$ & $\begin{array}{l}\text { Laboratory } \\
\text { No. }\end{array}$ & Remark & Collector & $\mathrm{SiO}_{2}$ & $\mathrm{Al}_{2} \mathrm{O}_{3}$ & $\mathrm{Fe}_{2} \mathrm{O}_{3}$ & $\mathrm{FeO}$ & $\mathrm{MgO}$ & $\mathrm{CaO}$ & $\mathrm{Na}_{2} \mathrm{O}$ & $\mathrm{K}_{2} \mathrm{O}$ & $\mathrm{H}_{2} \mathrm{O}$ & $\mathrm{TiO}_{2}$ & $\mathrm{P}_{2} \mathrm{O}_{5}$ & $\mathrm{MnO}$ & $\mathrm{CO}_{2}$ & Total \\
\hline GP-36 & MK & $\mathrm{I}$ & 1 & D-276075 & $\bar{b}$ & EWW & 49.10 & 17.00 & 12.40 & nd & 4.46 & 7.35 & 4.51 & 1.76 & .00 & 3.09 & .84 & .21 & nd & 100.72 \\
\hline GP-37 & MK & lc & 1 & D-276073 & b & EWW & 49.80 & 16.90 & 11.80 & nd & 4.03 & 7.04 & 4.56 & 1.90 & .00 & 2.76 & .96 & .22 & nd & 99.97 \\
\hline GP-38 & ML & K2 & 1 & D-276072 & b & EWW & 51.60 & 13.40 & 12.50 & nd & 7.43 & 10.70 & 2.04 & .33 & .00 & 2.20 & .23 & .17 & nd & 100.60 \\
\hline GQ-1 & ML & $\mathrm{k} 2$ & 1 & W-228255 & $b$ & JPL & 51.50 & 13.70 & 13.20 & nd & 6.28 & 10.60 & 2.38 & .50 & .00 & 2.48 & .31 & .18 & nd & 101.13 \\
\hline GQ-2 & ML & k4 & 1 & ML-109 & $\mathrm{a}$ & JPL & 51.95 & 13.80 & 11.74 & nd & 6.83 & 10.60 & 2.29 & .42 & nd & 2.04 & .25 & .17 & nd & 100.09 \\
\hline$G Q-3$ & ML & k & 1 & D-313819 & b & $J P L$ & 48.90 & 14.60 & 14.10 & nd & 5.58 & 10.30 & 3.05 & .63 & .00 & 3.23 & .41 & .19 & nd & 100.99 \\
\hline GQ-4 & ML & kc1o & 1 & D-313818 & b & JPL & 50.50 & 13.80 & 12.30 & nd & 7.99 & 10.70 & 2.30 & .41 & .00 & 2.22 & .27 & .16 & nd & 100.65 \\
\hline GQ-5 & ML & $k 5-1843$ & 1 & D-313814 & b & JPL & 51.70 & 13.80 & 12.30 & nd & 7.38 & 10.80 & 2.27 & .45 & .00 & 2.20 & .28 & .17 & nd & 101.35 \\
\hline GQ-6 & $\mathrm{ML}$ & k1o & 1 & D-313816 & b & JPL & 50.40 & 11.80 & 12.50 & nd & 12.00 & 9.54 & 1.92 & .34 & .00 & 2.11 & .23 & .17 & nd & 101.01 \\
\hline GQ-7 & $\mathrm{ML}$ & $k 5-1843$ & 1 & ML-108 & a & $J P L$ & 51.72 & 13.77 & 11.95 & nd & 6.78 & 10.52 & 2.26 & .47 & nd & 2.18 & .27 & .17 & nd & 100.09 \\
\hline GQ-9 & MK & lc & 1 & D-284776 & b & EWW & 50.20 & 17.00 & 12.00 & nd & 4.13 & 7.09 & 4.16 & 1.84 & .00 & 2.91 & .89 & .22 & nd & 100.44 \\
\hline GQ-10 & $\mathrm{ML}$ & k4 & 1 & NE-53 & a & JPL & 51.84 & 13.72 & 11.76 & nd & 6.95 & 10.56 & 2.45 & .40 & nd & 2.02 & .24 & .16 & nd & 100.10 \\
\hline GQ-11 & MK & 1 & 1 & W-235826 & b & $J P L$ & 49.25 & 17.59 & 11.54 & nd & 4.17 & 6.99 & 4.86 & 1.86 & nd & 2.67 & .77 & .22 & nd & 99.92 \\
\hline GQ-12 & ML & $k 2$ & 1 & W-235829 & $\mathrm{b}$ & JPL & 51.37 & 15.55 & 12.26 & nd & 7.13 & 10.47 & 2.85 & .44 & nd & 2.05 & .30 & .18 & nd & 102.60 \\
\hline GQ-13 & $\mathrm{ML}$ & $k 3$ & 1 & D-313817 & b & $J P L$ & 52.10 & 13.30 & 11.60 & nd & 8.01 & 10.50 & 2.35 & .43 & .00 & 2.46 & .27 & .16 & nd & 101.18 \\
\hline GQ-14 & ML & k4 & 1 & W-235827 & b & JPL & 51.40 & 14.27 & 11.79 & nd & 7.12 & 10.76 & 2.47 & .45 & nd & 1.98 & .29 & .18 & nd & 100.71 \\
\hline GQ-15 & ML & $\mathrm{k} 2$ & 1 & W-235823 & $b$ & $J P L$ & 48.98 & 14.09 & 12.25 & nd & 10.00 & 10.25 & 2.25 & .43 & nd & 2.03 & .28 & .17 & nd & 100.73 \\
\hline GQ-16 & ML & k4 & 1 & D-276062 & b & EWW & 52.40 & 13.70 & 12.20 & nd & 7.14 & 10.90 & 2.24 & .40 & .00 & 2.11 & .25 & .17 & nd & 101.51 \\
\hline GQ-17 & ML & k4 & 1 & D-313815 & b & JPL & 52.10 & 13.90 & 12.10 & nd & 6.93 & 10.90 & 2.38 & .40 & .00 & 2.13 & .25 & .17 & nd & 101.26 \\
\hline GQ-18 & MK & lc & 1 & D-276061 & $b$ & EWW & 50.20 & 17.00 & 11.60 & nd & 4.16 & 7.03 & 4.67 & 1.88 & .00 & 2.79 & .88 & .22 & nd & 100.43 \\
\hline GQ-19 & MK & lc & 1 & D-276060 & $b$ & EWW & 50.00 & 17.00 & 11.80 & nd & 4.18 & 7.08 & 4.82 & 1.88 & .00 & 2.83 & .90 & .22 & nd & 100.71 \\
\hline GQ-20 & ML & $k 2$ & 1 & D-276059 & b & EWW & 51.70 & 13.80 & 12.40 & nd & 7.04 & 10.70 & 2.39 & .39 & .00 & 2.10 & .25 & .17 & nd & 100.94 \\
\hline GQ-21 & MK & 1 & 1 & D-276074 & b & EWW & 48.50 & 16.70 & 12.30 & nd & 4.60 & 7.42 & 4.51 & 1.70 & .24 & 3.13 & .79 & .21 & nd & 100.10 \\
\hline GQ-22 & MK & 1 & 1 & D-284775 & $\mathrm{b}$ & EWW & 49.10 & 16.80 & 12.30 & nd & 4.47 & 7.37 & 4.29 & 1.72 & .00 & 3.11 & .81 & .21 & nd & 100.18 \\
\hline GQ-23 & MK & k & 1 & D-276063 & b & EWW & 48.60 & 16.80 & 12.40 & nd & 4.66 & 7.45 & 4.15 & 1.67 & .00 & 3.09 & .82 & .21 & nd & 99.85 \\
\hline GQ-24 & ML & $k 2$ & 1 & D-276058 & b & EWW & 49.50 & 13.40 & 12.70 & nd & 10.30 & 10.30 & 2.33 & .41 & .00 & 2.09 & .25 & .17 & nd & 101.45 \\
\hline GR-1 & ML & k5-1935 & 1 & ML-28 & a & JMR & 51.45 & 13.60 & 12.08 & nd & 7.01 & 10.57 & 2.13 & .37 & nd & 2.08 & .23 & .17 & nd & 99.69 \\
\hline GR-2 & ML & k5-1899 & 1 & D-313824 & b & JPL & 51.80 & 13.60 & 12.50 & nd & 7.22 & 10.90 & 2.21 & .35 & .00 & 2.64 & .24 & .18 & nd & 101.64 \\
\hline GR-3 & ML & $\mathrm{k} 4$ & 1 & W-214666 & c & JPL & 47.70 & 9.10 & 1.70 & 10.00 & 21.60 & 7.00 & 1.70 & .24 & .49 & 1.30 & .19 & .15 & .00 & 101.17 \\
\hline GR-4 & ML & $k 5-1880-81$ & 1 & D-313821 & b & JPL & 52.20 & 13.40 & 12.30 & nd & 8.08 & 10.50 & 2.19 & .33 & .00 & 2.03 & .22 & .17 & nd & 101.42 \\
\hline GR-5 & ML & k5-1855 & 1 & D-313820 & b & $J P L$ & 50.90 & 13.10 & 12.40 & nd & 8.99 & 10.30 & 2.17 & .41 & .00 & 2.12 & .26 & .17 & nd & 100.82 \\
\hline GR-6 & ML & k5-1935 & 1 & D-313822 & b & JPL & 51.70 & 13.70 & 12.40 & nd & 7.11 & 10.90 & 2.24 & .36 & .00 & 2.18 & .25 & .17 & nd & 101.01 \\
\hline GR-7 & ML & k1y & 1 & D-313823 & $\mathrm{b}$ & JPL & 50.10 & 11.80 & 12.70 & nd & 12.30 & 9.27 & 1.89 & .29 & .19 & 1.88 & .20 & .18 & nd & 100.80 \\
\hline GR-8 & ML & k5-1935 & 1 & ML-27 & a & JMR & 51.56 & 13.73 & 12.04 & nd & 7.03 & 10.50 & 2.37 & .38 & nd & 2.08 & .22 & .18 & nd & 100.09 \\
\hline GR-9 & ML & $\mathrm{k} 2$ & 1 & W-228257 & b & JPL & 51.90 & 13.90 & 12.90 & nd & 6.98 & 11.00 & 2.32 & .36 & .00 & 2.02 & .23 & .17 & nd & 101.78 \\
\hline GR-11 & ML & $k 2$ & 1 & D-284787 & b & EWW & 52.10 & 13.90 & 12.50 & nd & 6.94 & 10.70 & 2.34 & .39 & .00 & 2.12 & .25 & .18 & nd & 101.42 \\
\hline GR-12 & MK & lc & 1 & D-276026 & b & EWW & 49.30 & 16.90 & 12.00 & nd & 4.19 & 7.18 & 4.30 & 1.77 & .35 & 2.94 & .89 & .22 & nd & 100.04 \\
\hline GR-13 & MK & 1 & 1 & D-276025 & $\mathrm{b}$ & EWW & 48.50 & 16.80 & 12.50 & nd & 4.60 & 7.30 & 4.25 & 1.69 & .00 & 3.16 & .79 & .21 & nd & 99.80 \\
\hline GR-14 & MK & lc & 1 & D-276036 & b & EWW & 49.20 & 17.10 & 11.90 & nd & 3.87 & 6.95 & 4.20 & 1.77 & 1.16 & 2.76 & .94 & .22 & nd & 100.07 \\
\hline GR-15 & MK & ly & 1 & D-276035 & b & EWW & 50.60 & 17.00 & 11.60 & nd & 3.96 & 6.77 & 4.82 & 1.93 & .00 & 2.67 & .93 & .22 & nd & 100.50 \\
\hline
\end{tabular}


Table 1.-Chemical analyses-Continued

\begin{tabular}{|c|c|c|c|c|c|c|c|c|c|c|c|c|c|c|c|c|c|c|c|c|}
\hline $\begin{array}{c}\text { Sample } \\
\text { locality } \\
\text { No. }\end{array}$ & $\begin{array}{l}\text { Vol- } \\
\text { cano }\end{array}$ & Unit & $\begin{array}{c}\text { Map } \\
\text { sheet } \\
\text { No. }\end{array}$ & $\begin{array}{l}\text { Laboratory } \\
\text { No. }\end{array}$ & Remark & Collector & $\mathrm{SiO}_{2}$ & $\mathrm{Al}_{2} \mathrm{O}_{3}$ & $\mathrm{Fe}_{2} \mathrm{O}_{3}$ & $\mathrm{FeO}$ & $\mathrm{MgO}$ & $\mathrm{CaO}$ & $\mathrm{Na}_{2} \mathrm{O}$ & $\mathrm{K}_{2} \mathrm{O}$ & $\mathrm{H}_{2} \mathrm{O}$ & $\mathrm{TO}_{2}$ & $\mathrm{P}_{2} \mathrm{O}_{5}$ & $\mathrm{MnO}$ & $\mathrm{CO}_{2}$ & Total \\
\hline GR-16 & MK & Iy & 1 & D-276034 & $\mathrm{b}$ & EWW & 50.40 & 17.00 & 11.50 & nd & 4.02 & 6.81 & 4.60 & 1.90 & .00 & 2.67 & .91 & .22 & nd & 100.03 \\
\hline GR-17 & MK & lc & 1 & D-276024 & b & EWW & 49.30 & 16.90 & 12.10 & nd & 4.35 & 7.30 & 4.40 & 1.80 & .05 & 3.05 & .87 & .22 & nd & 100.34 \\
\hline GR-18 & MK & 1 & 1 & D-276041 & b & EWW & 50.60 & 17.40 & 10.70 & nd & 3.32 & 6.47 & 4.80 & 2.08 & .71 & 2.29 & 1.26 & .22 & nd & 99.85 \\
\hline GR-19 & MK & 1 & 1 & D-276027 & b & EWW & 48.70 & 17.00 & 12.10 & nd & 4.31 & 7.35 & 4.35 & 1.68 & .25 & 3.02 & .90 & .22 & nd & 99.88 \\
\hline GR-20 & MK & 1 & 1 & D-276042 & b & EWW & 50.50 & 17.00 & 11.30 & nd & 3.78 & 6.72 & 4.80 & 2.00 & .00 & 2.58 & 1.03 & .22 & nd & 99.93 \\
\hline GR-21 & MK & k & 1 & D-284786 & b & EWW & 50.30 & 17.10 & 11.90 & nd & 4.06 & 6.95 & 4.35 & 1.88 & .00 & 2.79 & .91 & .22 & nd & 100.46 \\
\hline GR-22 & MK & 1 & 1 & D-276043 & b & EWW & 49.80 & 16.90 & 11.80 & nd & 4.12 & 7.01 & 4.64 & 1.85 & .00 & 2.83 & .89 & .22 & nd & 100.06 \\
\hline GR-23 & MK & 1 & 1 & D-276029 & b & EWW & 49.80 & 17.00 & 11.90 & nd & 4.17 & 7.09 & 4.48 & 1.81 & .00 & 2.88 & .90 & .22 & nd & 100.25 \\
\hline GR-24 & MK & lc & 1 & D-276044 & b & EWW & 50.80 & 17.50 & 11.00 & nd & 3.28 & 6.33 & 4.73 & 2.04 & .28 & 2.33 & 1.15 & .22 & nd & 99.66 \\
\hline GR-25 & MK & ly & 1 & D-276037 & b & EWW & 50.70 & 17.00 & 11.60 & nd & 3.99 & 6.82 & 4.64 & 1.94 & .21 & 2.67 & .92 & .22 & nd & 100.71 \\
\hline GR-26 & MK & 1 & 1 & D-276030 & b & EWW & 49.40 & 16.90 & 12.20 & nd & 4.42 & 7.26 & 4.38 & 1.79 & .00 & 3.05 & .85 & .22 & nd & 100.47 \\
\hline GR-27 & MK & 1 & 1 & D-276028 & b & EWW & 49.80 & 17.00 & 12.10 & nd & 4.27 & 7.13 & 4.36 & 1.82 & .00 & 2.95 & .89 & .22 & nd & 100.54 \\
\hline GR-28 & MK & lc & 1 & D-276048 & b & EWW & 50.00 & 17.90 & 10.90 & nd & 3.39 & 6.56 & 4.50 & 1.74 & 1.24 & 2.34 & 1.28 & .23 & nd & 100.08 \\
\hline GR-29 & MK & lc & 1 & D-284782 & $b$ & EWW & 49.70 & 16.90 & 11.70 & nd & 4.09 & 7.05 & 4.45 & 1.85 & .58 & 2.77 & .89 & .22 & nd & 100.20 \\
\hline GR-30 & MK & ly & 1 & D-276040 & b & EWW & 50.30 & 16.90 & 11.60 & nd & 3.97 & 6.77 & 4.81 & 1.92 & .00 & 2.66 & .92 & .22 & nd & 100.07 \\
\hline GR-31 & MK & i & 1 & D-276049 & b & EWW & 51.10 & 17.20 & 11.00 & nd & 3.34 & 6.43 & 4.98 & 2.14 & .10 & 2.28 & 1.20 & .23 & nd & 100.00 \\
\hline GR-32 & MK & k & 1 & D-276031 & b & EWW & 49.40 & 16.90 & 12.00 & nd & 4.27 & 7.20 & 4.54 & 1.78 & .04 & 2.96 & .88 & .22 & nd & 100.19 \\
\hline GR-33 & MK & c & 1 & D-284783 & b & EWW & 49.80 & 17.00 & 11.90 & nd & 4.07 & 7.03 & 4.40 & 1.88 & .05 & 2.81 & .88 & .22 & nd & 100.04 \\
\hline GR-34 & MK & ly & 1 & D-276039 & b & EWW & 50.70 & 17.00 & 11.50 & nd & 3.97 & 6.87 & 4.67 & 1.95 & .00 & 2.68 & .93 & .22 & nd & 100.49 \\
\hline GR-35 & MK & 1 & 1 & D-276032 & b & EWW & 50.80 & 17.00 & 11.60 & nd & 3.95 & 6.95 & 4.66 & 1.94 & .00 & 2.72 & .94 & .22 & nd & 100.78 \\
\hline GR-36 & MK & 1 & 1 & D-276033 & b & EWW & 50.10 & 16.90 & 11.40 & nd & 3.96 & 6.92 & 4.70 & 1.96 & .04 & 2.69 & .93 & .22 & nd & 99.82 \\
\hline GR-37 & MK & ly & 1 & D-276038 & b & EWW & 50.70 & 16.90 & 11.50 & nd & 3.95 & 6.82 & 4.62 & 1.96 & .00 & 2.66 & .93 & .22 & nd & 100.26 \\
\hline GR-38 & MK & lc & 1 & D-276047 & b & EWW & 50.40 & 17.10 & 11.20 & nd & 3.81 & 6.85 & 4.82 & 2.00 & .02 & 2.62 & 1.02 & .22 & nd & 100.06 \\
\hline GR-39 & MK & lc & 1 & D-284781 & b & EWW & 50.50 & 17.10 & 11.60 & nd & 3.87 & 7.01 & 4.56 & 1.94 & .00 & 2.67 & .96 & .22 & nd & 100.43 \\
\hline GR-40 & MK & 1 & 1 & D-284780 & b & EWW & 50.30 & 16.90 & 11.40 & nd & 3.96 & 6.95 & 4.35 & 1.91 & .00 & 2.73 & .94 & .22 & nd & 99.66 \\
\hline GR-41 & MK & 1 & 1 & D-284779 & b & EWW & 50.80 & 17.20 & 11.20 & nd & 3.88 & 6.89 & 4.63 & 1.95 & .00 & 2.69 & .92 & .22 & nd & 100.38 \\
\hline GR-42 & MK & lc & 1 & D-284784 & $\mathrm{b}$ & EWW & 50.40 & 17.40 & 10.70 & nd & 3.56 & 6.73 & 4.55 & 2.04 & .88 & 2.48 & 1.05 & .21 & nd & 100.00 \\
\hline GR-43 & MK & ly & 1 & D-278148 & b & EWW & 52.00 & 17.20 & 10.80 & nd & 3.61 & 6.48 & 4.90 & 2.10 & .00 & 2.36 & .98 & .23 & nd & 100.66 \\
\hline GR-44 & MK & lc & 1 & D-284778 & $b$ & EWW & 50.00 & 16.90 & 11.90 & nd & 4.21 & 6.90 & 4.42 & 1.83 & .19 & 2.79 & .88 & .22 & nd & 100.24 \\
\hline GR-45 & MK & 1 & 1 & D-284785 & b & EWW & 48.70 & 17.10 & 12.10 & nd & 4.03 & 7.32 & 4.20 & 1.86 & .65 & 2.94 & .93 & .22 & nd & 100.05 \\
\hline GR-46 & MK & lc & 1 & D-284777 & b & EWW & 50.20 & 17.10 & 11.60 & nd & 3.82 & 6.89 & 4.40 & 1.85 & .22 & 2.66 & .96 & .22 & nd & 99.92 \\
\hline GS-1 & ML & k10 & 2 & D-313495 & b & JPL & 51.50 & 13.70 & 12.40 & nd & 7.07 & 10.70 & 2.23 & .41 & .35 & 2.19 & .26 & .17 & nd & 100.98 \\
\hline GS-2 & ML & k5-1984 & 2 & D-313494 & b & JPL & 51.80 & 13.50 & 12.30 & nd & 7.30 & 10.60 & 2.08 & .40 & .24 & 2.14 & .25 & .17 & nd & 100.78 \\
\hline GS-3 & ML & $k 5-1880-81$ & 2 & ML-26 & a & JMR & 51.78 & 13.25 & 11.96 & nd & 7.79 & 10.10 & 2.08 & .33 & nd & 1.95 & .22 & .16 & nd & 99.62 \\
\hline GS-4 & ML & k5-1855 & 2 & ML-25 & a & JMR & 50.82 & 13.28 & 12.10 & nd & 8.24 & 10.22 & 2.07 & .42 & nd & 2.10 & .24 & .17 & nd & 99.66 \\
\hline GS-5 & MK & ly & 2 & W-228261 & b & JPL & 49.70 & 16.80 & 13.50 & nd & 3.95 & 6.60 & 4.56 & 1.86 & .00 & 2.66 & .91 & .24 & nd & 100.78 \\
\hline GS-6 & ML & k2 & 2 & W-228265 & b & JPL & 51.60 & 13.50 & 13.40 & nd & 6.75 & 10.70 & 2.22 & .38 & .00 & 2.19 & .25 & .18 & nd & 101.17 \\
\hline GS-7 & ML & k1y & 2 & D-313493 & b & JPL & 51.70 & 13.20 & 11.70 & nd & 9.00 & 10.30 & 2.12 & .33 & .07 & 2.11 & .22 & .16 & nd & 100.91 \\
\hline GS-8 & ML & k1y & 2 & W-228274 & b & JPL & 50.60 & 12.30 & 13.20 & nd & 10.90 & 9.84 & 2.00 & .31 & .00 & 1.91 & .21 & .17 & nd & 101.44 \\
\hline GS-9 & MK & $\mathrm{hm}$ & 2 & D-276045 & b & EWW & 46.40 & 14.10 & 14.80 & nd & 6.21 & 10.30 & 2.96 & .99 & .10 & 4.05 & .56 & .20 & nd & 100.67 \\
\hline
\end{tabular}


Table 1.-Chemical analyses-Continued

\begin{tabular}{|c|c|c|c|c|c|c|c|c|c|c|c|c|c|c|c|c|c|c|c|c|}
\hline $\begin{array}{c}\text { Sample } \\
\text { locality } \\
\text { No. }\end{array}$ & $\begin{array}{l}\text { Vol- } \\
\text { cano }\end{array}$ & Unit & $\begin{array}{l}\text { Map } \\
\text { sheet } \\
\text { No. }\end{array}$ & $\begin{array}{l}\text { Laboratory } \\
\text { No. }\end{array}$ & Remark & Collector & $\mathrm{SiO}_{2}$ & $\mathrm{Al}_{2} \mathrm{O}_{3}$ & $\mathrm{Fe}_{2} \mathrm{O}_{3}$ & $\mathrm{FeO}$ & $\mathrm{MgO}$ & $\mathrm{CaO}$ & $\mathrm{Na}_{2} \mathrm{O}$ & $\mathrm{K}_{2} \mathrm{O}$ & $\mathrm{H}_{2} \mathrm{O}$ & $\mathrm{TOO}_{2}$ & $\mathrm{P}_{2} \mathrm{O}_{5}$ & $\mathrm{MnO}$ & $\mathrm{CO}_{2}$ & Total \\
\hline GS-10 & $\overline{\mathrm{ML}}$ & $k 1 y$ & 2 & W-228275 & $\mathrm{b}$ & JPL & 51.10 & 13.50 & 14.40 & nd & 7.05 & 10.60 & 2.30 & .41 & .00 & 2.12 & .27 & .18 & nd & 101.93 \\
\hline GS-11 & MK & $\mathrm{hm}$ & 2 & D-276046 & b & EWW & 44.80 & 14.60 & 13.20 & nd & 7.90 & 12.30 & 1.97 & .22 & 1.84 & 3.08 & .37 & .18 & nd & 100.46 \\
\hline GS-12 & MK & $\mathrm{hm}$ & 2 & W-228276 & b & JPL & 45.10 & 13.60 & 14.20 & nd & 9.76 & 11.20 & 2.10 & .39 & 1.06 & 2.98 & .38 & .18 & nd & 100.95 \\
\hline GS-13 & ML & k & 2 & W-228277 & b & JPL & 51.40 & 13.50 & 13.30 & nd & 6.93 & 10.60 & 2.33 & .42 & .00 & 2.27 & .27 & .17 & nd & 101.19 \\
\hline GS-14 & $\mathrm{ML}$ & $\ddot{k}$ & 2 & W-214663 & c & JPL & 51.80 & 13.10 & 2.60 & 8.70 & 8.20 & 10.00 & 2.40 & .37 & .32 & 2.00 & .28 & .13 & .00 & 99.90 \\
\hline GS-15 & MK & I & 2 & D-276050 & b & EWW & 50.50 & 17.20 & 11.20 & nd & 3.56 & 6.70 & 4.92 & 2.06 & .06 & 2.42 & 1.14 & .22 & nd & 99.98 \\
\hline GS-16 & ML & k & 2 & W-214683 & c & JPL & 52.00 & 14.00 & 1.80 & 9.10 & 6.80 & 10.00 & 2.30 & .45 & .88 & 1.70 & .31 & .17 & .11 & 99.62 \\
\hline GT-2 & ML & k4 & 2 & NE-19 & a & JPL & 52.31 & 14.09 & 11.45 & nd & 6.96 & 10.67 & 2.18 & .44 & nd & 2.14 & .28 & .17 & nd & 100.69 \\
\hline GT-4 & $\mathrm{ML}$ & $k 2$ & 2 & D-313836 & b & JPL & 51.60 & 13.60 & 12.80 & nd & 6.65 & 10.70 & 2.26 & .42 & .00 & 2.27 & .28 & .17 & nd & 100.75 \\
\hline GT-6 & ML & $\mathrm{kB}$ & 2 & D-313835 & b & JPL & 52.10 & 13.30 & 12.50 & nd & 7.35 & 10.70 & 2.15 & .41 & .00 & 2.18 & .25 & .17 & nd & 101.11 \\
\hline GT-7 & $\mathrm{ML}$ & k10 & 2 & W-214661 & c & JPL & 51.50 & 13.20 & 2.60 & 8.70 & 9.90 & 9.50 & 2.40 & .34 & .70 & 1.90 & .25 & .14 & .00 & 101.13 \\
\hline GT-8 & ML & k1o & 2 & W-214660 & c & JPL & 51.60 & 13.80 & 2.70 & 8.80 & 7.00 & 9.60 & 2.50 & .42 & .39 & 2.30 & .34 & .12 & .00 & 99.57 \\
\hline GT-11 & ML & k1o & 2 & NE-22 & a & JMR & 51.75 & 13.97 & 12.23 & nd & 7.11 & 10.19 & 2.10 & .40 & nd & 2.22 & .28 & .17 & nd & 100.42 \\
\hline GT-13 & ML & k10 & 2 & W-214662 & c & JPL & 48.20 & 8.50 & 1.80 & 9.50 & 22.60 & 6.40 & 1.60 & .22 & .42 & 1.20 & .17 & .13 & .00 & 100.74 \\
\hline GT-15 & ML & k1y & 2 & D-313834 & b & JPL & 51.40 & 13.70 & 12.70 & nd & 6.94 & 10.50 & 2.16 & .40 & .50 & 2.30 & .28 & .18 & nd & 101.06 \\
\hline GT-16 & $\mathrm{ML}$ & k5-1880-81 & 2 & W-214659 & c & JPL & 52.60 & 13.20 & 2.30 & 8.90 & 8.20 & 10.10 & 2.40 & .37 & .36 & 2.00 & .26 & .12 & .00 & 100.81 \\
\hline GT-17 & $\mathrm{ML}$ & k5-1855 & 2 & ML-24 & a & JMR & 51.17 & 13.55 & 12.02 & nd & 7.37 & 10.42 & 2.12 & .43 & nd & 2.14 & .26 & .16 & nd & 99.64 \\
\hline GT-18 & MK & $\mathrm{hm}$ & 2 & W-227769 & c & JBB & 44.20 & 14.20 & 5.20 & 10.00 & 6.00 & 9.00 & 2.10 & .76 & 2.85 & 3.70 & .51 & .22 & .02 & 98.76 \\
\hline GT-19 & ML & k & 2 & D-313833 & b & JPL & 51.30 & 13.50 & 12.60 & nd & 7.26 & 10.40 & 2.23 & .41 & .75 & 2.23 & .26 & .18 & nd & 101.12 \\
\hline GT-21 & ML & k1y & 2 & W-214658 & c & JPL & 51.80 & 13.00 & 2.90 & 8.20 & 7.20 & 10.20 & 2.60 & .41 & .62 & 2.10 & .30 & .12 & .00 & 99.45 \\
\hline GU-1 & ML & $\mathbf{k} 3$ & 2 & W-227788 & c & JBB & 48.90 & 11.80 & 2.00 & 9.40 & 13.20 & 8.90 & 1.80 & .22 & .46 & 1.60 & .24 & .17 & .07 & 98.76 \\
\hline GU-2 & ML & k10 & 2 & W-220497 & c & $\mathrm{JBB}$ & 50.80 & 13.80 & 2.60 & 8.40 & 9.00 & 9.90 & 2.20 & .32 & 1.01 & 2.00 & .29 & .16 & .04 & 100.52 \\
\hline GU-4 & ML & k10 & 2 & W-227790 & c & JBB & 47.40 & 9.80 & 2.30 & 9.70 & 18.40 & 7.50 & 1.50 & .14 & .74 & 1.40 & .18 & .17 & .03 & 99.26 \\
\hline GU-5 & ML & $\mathrm{kB}$ & 2 & W-227780 & c & JBB & 50.20 & 12.10 & 2.90 & 8.20 & 11.30 & 9.40 & 1.90 & .28 & .56 & 1.70 & .24 & .16 & .08 & 99.02 \\
\hline GU-6 & ML & $\mathbf{k}$ & 2 & NE-9 & a & JPL & 50.48 & 12.49 & 11.61 & nd & 10.64 & 9.67 & 2.10 & .35 & nd & 1.81 & .23 & .18 & nd & 99.56 \\
\hline GU-8 & ML & $k 10$ & 2 & NE-20 & a & JMR & 48.16 & 9.98 & 12.42 & nd & 17.83 & 7.73 & 1.39 & .25 & nd & 1.53 & .17 & .17 & nd & 99.63 \\
\hline GU-9 & ML & k10 & 2 & W-229943 & c & $\mathrm{JBB}$ & 50.00 & 13.80 & 3.10 & 8.60 & 6.80 & 9.60 & 2.20 & .61 & 1.40 & 2.30 & .29 & .17 & .02 & 98.89 \\
\hline GU-10 & ML & $k 10$ & 2 & W-227784 & c & JBB & 45.90 & 8.00 & 1.90 & 9.80 & 24.00 & 6.50 & 1.10 & .12 & 1.20 & 1.10 & .15 & .17 & .06 & 100.00 \\
\hline GU-11 & ML & $\mathrm{K} 3$ & 2 & W-227779 & c & JBB & 49.90 & 12.70 & 2.10 & 8.90 & 10.70 & 9.80 & 2.00 & .30 & .53 & 1.80 & .26 & .16 & .07 & 99.22 \\
\hline GU-13 & ML & $\mathrm{k} 3$ & 2 & W-214535 & c & JBB & 51.90 & 13.70 & 2.40 & 8.60 & 7.20 & 10.20 & 2.10 & .41 & .48 & 2.10 & .27 & .17 & .01 & 99.54 \\
\hline GU-14 & ML & k & 2 & W-218522 & c & JBB & 50.80 & 13.90 & 3.00 & 8.70 & 6.80 & 9.80 & 2.30 & .14 & .99 & 2.20 & .25 & .17 & .06 & 99.11 \\
\hline GU-15 & $\mathrm{ML}$ & k10 & 2 & W-227776 & c & JBB & 50.80 & 13.20 & 3.10 & 8.50 & 7.70 & 10.20 & 2.00 & .32 & .71 & 2.00 & .25 & .17 & .09 & 99.04 \\
\hline GU-16 & ML & k10 & 2 & W-227785 & c & JBB & 47.50 & 10.10 & 2.20 & 9.00 & 17.20 & 8.40 & 1.50 & .16 & .94 & 1.40 & .20 & .17 & .08 & 98.85 \\
\hline GU-17 & $\mathrm{ML}$ & k10 & 2 & W-240446 & c & JBB & 48.20 & 10.80 & 2.50 & 9.20 & 18.20 & 7.60 & 1.60 & .27 & .55 & 1.50 & .20 & .17 & .01 & 100.80 \\
\hline GU-18 & ML & $k 10$ & 2 & W-229948 & c & JBB & 47.80 & 8.70 & 2.60 & 8.40 & 21.40 & 7.50 & 1.10 & .19 & .50 & 1.10 & .15 & .16 & .01 & 99.61 \\
\hline GU-19 & ML & $\mathrm{K} 3$ & 2 & W-227778 & c & JBB & 49.90 & 12.60 & 2.30 & 8.90 & 10.30 & 9.80 & 2.00 & .34 & .39 & 1.80 & .25 & .16 & .05 & 98.79 \\
\hline GU-20 & ML & $k 10$ & 2 & W-240445 & c & JBB & 48.50 & 12.40 & 3.10 & 9.00 & 14.40 & 8.60 & 1.70 & .24 & .92 & 1.80 & .16 & .18 & .01 & 101.01 \\
\hline GU-21 & ML & $k 10$ & 2 & W-227775 & c & JBB & 48.10 & 10.70 & 2.20 & 10.00 & 16.60 & 8.00 & 1.60 & .20 & .57 & 1.60 & .20 & .17 & .06 & 100.00 \\
\hline GU-22 & $\mathrm{ML}$ & k10 & 2 & W-229950 & c & JBB & 47.20 & 8.10 & 2.30 & 8.80 & 22.40 & 7.50 & 1.10 & .11 & .80 & 1.10 & .13 & .17 & .01 & 99.72 \\
\hline GU-24 & ML & k10 & 2 & W-229947 & c & JBB & 47.70 & 10.60 & 5.20 & 6.50 & 15.80 & 8.00 & 1.40 & .22 & 1.40 & 1.60 & .20 & .17 & .01 & 98.80 \\
\hline
\end{tabular}


Table 1.-Chemical analyses-Continued

\begin{tabular}{|c|c|c|c|c|c|c|c|c|c|c|c|c|c|c|c|c|c|c|c|c|}
\hline $\begin{array}{c}\text { Sample } \\
\text { locality } \\
\text { No. }\end{array}$ & $\begin{array}{l}\text { Vol- } \\
\text { cano }\end{array}$ & Unit & $\begin{array}{c}\text { Map } \\
\text { sheet } \\
\text { No. }\end{array}$ & $\begin{array}{l}\text { Laboratory } \\
\text { No. }\end{array}$ & Remark & Collector & $\mathrm{SiO}_{2}$ & $\mathrm{Al}_{2} \mathrm{O}_{3}$ & $\mathrm{Fe}_{2} \mathrm{O}_{3}$ & $\mathrm{FeO}$ & $\mathrm{MgO}$ & $\mathrm{CaO}$ & $\mathrm{Na}_{2} \mathrm{O}$ & $\mathrm{K}_{2} \mathrm{O}$ & $\mathrm{H}_{2} \mathrm{O}$ & $\mathrm{TiO}_{2}$ & $\mathrm{P}_{2} \mathrm{O}_{5}$ & $\mathrm{MnO}$ & $\mathrm{CO}_{2}$ & Total \\
\hline GU-26 & ML & $\mathrm{k} 10$ & 2 & W-227787 & c & JBB & 50.60 & 13.50 & 2.30 & 9.20 & 7.90 & 10.00 & 2.20 & .24 & .56 & 2.00 & .28 & .17 & .08 & 99.03 \\
\hline GU-27 & ML & k1o & 2 & W-218526 & c & JBB & 49.50 & 11.60 & 2.20 & 9.40 & 12.60 & 8.60 & 1.90 & .12 & .81 & 1.80 & .21 & .16 & .05 & 98.95 \\
\hline GU-28 & ML. & k10 & 2 & W-215331 & c & JBB & 48.40 & 9.10 & 1.70 & 9.40 & 20.30 & 7.50 & 1.20 & .26 & .44 & 1.30 & .13 & .21 & .08 & 100.02 \\
\hline GU-29 & ML & k1y & 2 & W-218525 & c & JBB & 51.40 & 14.00 & 2.30 & 8.80 & 7.40 & 10.00 & 2.30 & .20 & .68 & 2.00 & .26 & .17 & .02 & 99.53 \\
\hline GU-30 & ML & $\mathrm{k} 3$ & 2 & W-240444 & c & JBB & 51.20 & 13.80 & 4.10 & 7.80 & 7.10 & 10.10 & 2.30 & .46 & .15 & 2.20 & .30 & .18 & .01 & 99.70 \\
\hline GU-31 & ML & k10 & 2 & W-229945 & c & JBB & 49.80 & 12.00 & 2.30 & 9.00 & 11.80 & 9.70 & 1.70 & .01 & 1.10 & 1.80 & .17 & .16 & .01 & 99.55 \\
\hline GU-34 & ML & k1y & 2 & W-215327 & c & JBB & 51.60 & 14.10 & 2.00 & 8.80 & 7.20 & 10.40 & 2.10 & .41 & .58 & 1.70 & .29 & .19 & .01 & 99.38 \\
\hline GU-35 & ML & k1y & 2 & W-227768 & c & JBB & 50.70 & 13.80 & 1.40 & 10.00 & 7.30 & 10.20 & 2.20 & .36 & 1.10 & 2.10 & .30 & .17 & .04 & 99.67 \\
\hline GU-36 & ML & $\mathrm{k} 3$ & 2 & W-227782 & c & JBB & 51.20 & 13.60 & 2.30 & 8.80 & 7.50 & 10.40 & 2.20 & .32 & .16 & 2.00 & .27 & .17 & .03 & 98.95 \\
\hline GU-37 & ML & k1o & 2 & W-227781 & c & JBB & 48.50 & 11.60 & 2.90 & 9.20 & 13.80 & 8.60 & 1.70 & .18 & .34 & 1.70 & .26 & .18 & .03 & 98.99 \\
\hline GU-38 & ML & $k 5-1880-81$ & 2 & ML-23 & $\mathbf{a}$ & JMR & 51.95 & 13.46 & 11.98 & nd & 7.28 & 10.30 & 2.14 & .35 & nd & 2.01 & .21 & .16 & nd & 99.84 \\
\hline GU-39 & ML & $k 5-1880-81$ & 2 & W-215326 & c & JBB & 52.40 & 13.80 & 1.70 & 9.10 & 8.00 & 9.80 & 2.20 & .34 & .36 & 1.70 & .26 & .18 & .01 & 99.85 \\
\hline GU-40 & ML & k1o & 2 & W-215334 & c & JBB & 50.50 & 12.50 & 2.40 & 8.40 & 11.00 & 9.20 & 1.90 & .31 & .80 & 1.80 & .14 & .20 & .01 & 99.16 \\
\hline GU-41 & ML & $k 3$ & 2 & W-215333 & c & JBB & 53.60 & 14.60 & 2.10 & 9.50 & 7.40 & 7.00 & 2.10 & .44 & .43 & 1.90 & .18 & .23 & .05 & 99.53 \\
\hline GU-42 & ML & $\mathrm{k} 3$ & 2 & NE-24 & a & JMR & 51.67 & 13.94 & 11.75 & nd & 7.29 & 10.63 & 2.12 & .36 & nd & 2.04 & .24 & .18 & nd & 100.22 \\
\hline GU-43 & ML & $\mathrm{k} 3$ & 2 & W-229946 & c & JBB & 50.70 & 13.60 & 2.30 & 9.40 & 7.20 & 10.20 & 2.20 & .43 & .81 & 2.10 & .25 & .17 & .01 & 99.37 \\
\hline GU-44 & ML & $\mathrm{kc}$ & 2 & W-231912 & c & JBB & 46.90 & 11.60 & 5.80 & 6.00 & 14.20 & 8.60 & 1.60 & .10 & 1.54 & 1.90 & .22 & .17 & .01 & 98.64 \\
\hline GU-45 & ML & $\mathrm{kc}$ & 2 & W-214537 & c & JBB & 47.10 & 11.00 & 1.90 & 9.40 & 17.80 & 8.20 & 1.50 & .14 & .99 & 1.60 & .23 & .16 & .01 & 100.03 \\
\hline GU-47 & ML & $k$ & 2 & W-214533 & c & JBB & 50.70 & 13.80 & 3.80 & 7.90 & 7.20 & 9.90 & 2.10 & .45 & 1.55 & 2.10 & .29 & .18 & .01 & 99.98 \\
\hline GU-48 & ML & $k$ & 2 & W-231911 & c & JBB & 49.10 & 13.80 & 2.60 & 8.80 & 7.20 & 9.20 & 1.90 & .38 & 3.20 & 2.10 & .28 & .17 & .03 & 98.76 \\
\hline GU-49 & ML & k1o & 2 & W-214536 & c & JBB & 51.10 & 13.70 & 2.90 & 8.30 & 7.40 & 10.00 & 2.10 & .38 & .73 & 2.10 & .26 & .16 & .01 & 99.14 \\
\hline GU-51 & ML & k & 2 & W-215330 & c & JBB & 52.10 & 14.50 & 2.00 & 9.20 & 7.20 & 10.00 & 2.00 & .42 & 1.49 & 1.70 & .23 & .20 & .04 & 101.08 \\
\hline GU-52 & ML & k & 2 & W-215329 & c & JBB & 51.40 & 13.30 & 2.30 & 8.60 & 9.40 & 9.60 & 1.80 & .33 & .60 & 1.80 & .23 & .20 & .01 & 99.57 \\
\hline GU-53 & MK & $\mathrm{hm}$ & 2 & W-214528 & c & JBB & 45.10 & 15.80 & 4.30 & 10.50 & 5.80 & 7.90 & 2.60 & .89 & 1.53 & 4.30 & .49 & .20 & .01 & 99.42 \\
\hline GU-54 & ML & k3 & 2 & W-215328 & c & JBB & 50.80 & 13.90 & 1.30 & 9.70 & 6.40 & 11.60 & 2.20 & .43 & .48 & 1.80 & .17 & .21 & .01 & 99.00 \\
\hline GU-58 & MK & $\mathrm{hm}$ & 2 & W-214530 & c & JBB & 45.60 & 14.70 & 3.90 & 10.00 & 5.70 & 9.90 & 2.70 & .84 & .77 & 4.20 & .43 & .20 & .01 & 98.95 \\
\hline GU-60 & $\mathrm{ML}$ & k & 2 & W-220495 & c & JBB & 51.60 & 14.80 & 2.00 & 8.80 & 7.80 & 9.60 & 2.30 & .41 & 1.66 & 2.00 & .30 & .14 & .06 & 101.47 \\
\hline GU-61 & ML & $\mathrm{k} 3$ & 2 & W-227792 & c & JBB & 50.60 & 13.00 & 2.50 & 9.00 & 9.70 & 9.80 & 2.10 & .24 & .31 & 1.90 & .27 & .17 & .06 & 99.65 \\
\hline GU-62 & ML & $\mathrm{k} 3$ & 2 & W-227793 & c & JBB & 51.10 & 13.60 & 2.40 & 9.70 & 6.70 & 10.10 & 2.40 & .30 & .50 & 2.20 & .32 & .18 & .08 & 99.58 \\
\hline GU-63 & ML & $k 3$ & 2 & W-227786 & c & JBB & 49.60 & 12.70 & 1.60 & 9.40 & 10.90 & 9.60 & 2.00 & .24 & .36 & 1.70 & .25 & .16 & .07 & 98.58 \\
\hline $\mathrm{HM}-1$ & HU & h1o & 1 & W-217433 & c & DAC & 45.80 & 14.10 & 3.10 & 9.40 & 10.60 & 9.80 & 2.50 & .73 & .47 & 2.00 & .28 & .19 & .02 & 98.99 \\
\hline HM-2 & $\mathrm{HU}$ & h1o & 1 & W-217435 & c & DAC & 46.20 & 14.60 & 6.30 & 6.00 & 9.30 & 10.60 & 2.80 & .70 & .56 & 1.90 & .27 & .16 & .01 & 99.40 \\
\hline HM-3 & $\mathrm{HU}$ & h5-1800-01 & 1 & W-217436 & c & DAC & 46.40 & 14.00 & 4.30 & 7.70 & 9.60 & 9.80 & 2.70 & .85 & .58 & 2.10 & .34 & .18 & .01 & 98.56 \\
\hline HM-4 & $\mathrm{HU}$ & h1o & 1 & W-227915 & c & DAC & 47.20 & 14.70 & 2.40 & 10.20 & 9.10 & 9.90 & 2.90 & 1.00 & .23 & 2.30 & .44 & .18 & .02 & 100.57 \\
\hline HM-5 & $\mathrm{HU}$ & h1y & 1 & W-217430 & c & DAC & 46.50 & 14.10 & 3.20 & 9.00 & 9.00 & 11.10 & 2.40 & .73 & .43 & 2.00 & .28 & .19 & .02 & 98.95 \\
\hline HM-6 & $\mathrm{HU}$ & hiy & 1 & W-217429 & c & DAC & 46.70 & 14.60 & 3.10 & 9.40 & 6.70 & 10.90 & 2.60 & .84 & .98 & 2.20 & .31 & .18 & .02 & 98.53 \\
\hline $\mathrm{HM}-7$ & HU & h1y & 1 & W-217428 & c & DAC & 46.20 & 13.50 & 8.20 & 4.70 & 10.40 & 10.60 & 2.50 & .73 & .26 & 1.90 & .27 & .20 & .01 & 99.47 \\
\hline HM-8 & $\mathrm{HU}$ & hc & 1 & W-217512 & c & $\mathrm{RBM}+\mathrm{DAC}$ & 47.00 & 13.80 & 6.30 & 5.50 & 8.00 & 11.50 & 2.50 & .70 & .97 & 2.10 & .30 & .16 & .02 & 98.85 \\
\hline HM-10 & HU & hc & 1 & W-217425 & c & $\mathrm{RBM}$ & 47.80 & 14.90 & 4.90 & 8.20 & 5.80 & 9.40 & 3.00 & 1.20 & .68 & 2.90 & .41 & .18 & .04 & 99.41 \\
\hline $\mathrm{HN}-1$ & $\mathrm{HU}$ & hc2 & 1 & W-217440 & c & DAC & 47.30 & 12.50 & 2.90 & 8.80 & 12.70 & 9.80 & 2.00 & .48 & .51 & 1.50 & .18 & .15 & .01 & 98.83 \\
\hline
\end{tabular}


Table 1.-Chemical analyses-Continued

\begin{tabular}{|c|c|c|c|c|c|c|c|c|c|c|c|c|c|c|c|c|c|c|c|c|}
\hline $\begin{array}{c}\text { Sample } \\
\text { locality } \\
\text { No. }\end{array}$ & $\begin{array}{l}\text { Vol- } \\
\text { cano }\end{array}$ & Unit & $\begin{array}{l}\text { Map } \\
\text { sheet } \\
\text { No. }\end{array}$ & $\begin{array}{l}\text { Laboratory } \\
\text { No. }\end{array}$ & Remark & Collector & $\mathrm{SiO}_{2}$ & $\mathrm{Al}_{2} \mathrm{O}_{3}$ & $\mathrm{Fe}_{2} \mathrm{O}_{3}$ & $\mathrm{FeO}$ & $\mathrm{MgO}$ & $\mathrm{CaO}$ & $\mathrm{Na}_{2} \mathrm{O}$ & $\mathrm{K}_{2} \mathrm{O}$ & $\mathrm{H}_{2} \mathrm{O}$ & $\mathrm{TiO}_{2}$ & $\mathrm{P}_{2} \mathrm{O}_{5}$ & $\mathrm{MnO}$ & $\mathrm{CO}_{2}$ & Total \\
\hline $\mathrm{HN}-2$ & $\mathrm{HU}$ & hc1y & 1 & W-217526 & c & $\overline{\mathrm{DAC}}$ & 47.20 & 14.00 & 3.10 & 9.20 & 9.10 & 10.50 & 2.50 & .83 & .52 & 2.10 & .30 & .19 & .01 & 99.55 \\
\hline $\mathrm{HN}-3$ & $\mathrm{HU}$ & hc1y & 1 & W-217441 & c & DAC & 44.70 & 13.40 & 4.10 & 8.50 & 12.40 & 9.30 & 2.20 & .62 & .99 & 1.90 & .27 & .15 & .04 & 98.57 \\
\hline HN-4 & $\mathrm{HU}$ & hc1y & 1 & W-217528 & c & DAC & 47.30 & 13.90 & 4.80 & 7.40 & 10.70 & 11.40 & 1.70 & .48 & .57 & 1.90 & .27 & .18 & .01 & 100.61 \\
\hline HN-5 & $\mathrm{HU}$ & hc1y & 1 & W-217442 & c & DAC & 45.70 & 13.70 & 3.00 & 9.20 & 10.00 & 10.20 & 2.50 & .86 & .42 & 2.40 & .30 & .18 & .01 & 98.47 \\
\hline HN-6 & $\mathrm{HU}$ & hc1y & 1 & W-214755 & c & RBM & 47.10 & 13.60 & 4.10 & 7.50 & 10.50 & 10.40 & 2.40 & .65 & .27 & 1.80 & .27 & .19 & .02 & 98.80 \\
\hline HN-7 & $\mathrm{HU}$ & h5-1800-01 & 1 & W-217531 & c & DAC & 46.90 & 14.40 & 2.00 & 10.30 & 10.00 & 11.00 & 2.40 & .77 & .53 & 2.10 & .30 & .19 & .02 & 100.91 \\
\hline HN-8 & $\mathrm{HU}$ & h1y & 1 & W-227902 & c & DAC & 45.60 & 12.80 & 2.30 & 9.10 & 11.70 & 11.30 & 2.20 & .80 & .47 & 2.20 & .34 & .17 & .03 & 99.01 \\
\hline $\mathrm{HN}-9$ & $\mathrm{HU}$ & hc1y & 1 & W-214756 & c & RBM & 46.90 & 13.20 & 2.50 & 9.40 & 10.70 & 10.40 & 2.40 & .80 & .26 & 2.00 & .30 & .20 & .02 & 99.08 \\
\hline $\mathrm{HN}-10$ & $\mathrm{HU}$ & hc & 1 & W-214757 & c & RBM & 46.30 & 15.70 & 3.70 & 10.30 & 5.80 & 10.50 & 2.80 & .93 & .44 & 2.70 & .31 & .20 & .02 & 99.70 \\
\hline HN-11 & $\mathrm{HU}$ & hc1o & 1 & W-214759 & c & RBM & 45.80 & 13.40 & 5.60 & 7.40 & 10.40 & 9.30 & 2.40 & .84 & .67 & 2.20 & .30 & .19 & .02 & 98.52 \\
\hline $\mathrm{HN}-12$ & $\mathrm{HU}$ & hc10 & 1 & W-214758 & c & RBM & 46.80 & 13.00 & 2.90 & 8.60 & 11.70 & 10.40 & 2.20 & .63 & .28 & 1.70 & .25 & .19 & .02 & 98.67 \\
\hline $\mathrm{HN}-13$ & $\mathrm{HU}$ & hc10 & 1 & W-214761 & c & $\mathrm{RBM}$ & 46.30 & 15.20 & 8.40 & 4.60 & 6.50 & 10.80 & 2.70 & 1.41 & .69 & 2.50 & .32 & .19 & .02 & 99.63 \\
\hline $\mathrm{HN}-14$ & $\mathrm{HU}$ & hc & 1 & W-214760 & c & RBM & 46.20 & 14.80 & 11.70 & 2.50 & 6.20 & 9.50 & 2.70 & .96 & .63 & 2.90 & .37 & .22 & .02 & 98.70 \\
\hline HN-15 & $\mathrm{HU}$ & h2 & 1 & W-227895 & c & DAC & 46.80 & 15.00 & 2.90 & 9.50 & 8.70 & 10.50 & 2.70 & .86 & .69 & 2.40 & .36 & .21 & .03 & 100.65 \\
\hline HN-16 & $\mathrm{HU}$ & h1y & 1 & W-227896 & c & DAC & 47.80 & 14.50 & 3.70 & 9.10 & 8.60 & 11.40 & 2.50 & .67 & .29 & 2.10 & .27 & .22 & .03 & 101.18 \\
\hline HN-17 & $\mathrm{HU}$ & h10 & 1 & W-227897 & c & DAC & 47.40 & 15.50 & 4.70 & 7.30 & 7.60 & 11.10 & 2.70 & .82 & .53 & 2.20 & .33 & .17 & .08 & 100.43 \\
\hline $\mathrm{HN}-18$ & HU & h2 & 1 & W-227898 & "c & DAC & 47.40 & 14.50 & 2.60 & 10.00 & 9.50 & 10.10 & 2.60 & .75 & .22 & 2.10 & .30 & .20 & .04 & 100.31 \\
\hline HN-19 & $\mathrm{ML}$ & $k 5-1859$ & 1 & ML-60 & $\mathrm{a}$ & JMR & 51.05 & 13.58 & 11.96 & nd & 7.42 & 10.55 & 2.17 & .43 & nd & 2.14 & .26 & .16 & nd & 99.72 \\
\hline $\mathrm{HN}-20$ & $\mathrm{HU}$ & h1o & 1 & W-227899 & c & DAC & 46.90 & 13.60 & 2.30 & 8.70 & 10.50 & 10.60 & 2.40 & .69 & .35 & 2.00 & .32 & .17 & .02 & 98.55 \\
\hline $\mathrm{HO}-1$ & $\mathrm{HU}$ & hc1o & 1 & W-227931 & c & $\mathrm{RBM}+\mathrm{DAC}$ & 47.80 & 13.90 & 3.70 & 7.90 & 9.60 & 11.40 & 2.30 & .70 & .27 & 1.80 & .31 & .17 & .01 & 99.86 \\
\hline $\mathrm{HO}-2$ & $\mathrm{HU}$ & hc2 & 1 & W-227933 & c & $\mathrm{RBM}+\mathrm{DAC}$ & 46.70 & 14.40 & 3.10 & 9.70 & 8.80 & 10.40 & 2.60 & .94 & .13 & 2.20 & .62 & .19 & .01 & 99.79 \\
\hline HO-3 & $\mathrm{HU}$ & hc1o & 1 & W-217450 & c & $\mathrm{RBM}$ & 47.40 & 13.10 & 9.60 & 4.10 & 10.40 & 11.00 & 1.90 & .71 & .79 & 1.80 & .29 & .17 & .01 & 101.27 \\
\hline $\mathrm{HO}-4$ & $\mathrm{HU}$ & hc2 & 1 & W-227934 & c & RBM & 46.60 & 14.40 & 2.30 & 10.40 & 8.70 & 10.50 & 2.50 & .90 & .28 & 2.20 & .37 & .18 & .07 & 99.40 \\
\hline HO-5 & HU & $\mathrm{h}$ & 1 & W-214688 & c & RBM & 46.20 & 11.80 & 1.50 & 10.40 & 13.50 & 10.10 & 2.20 & .65 & .26 & 1.90 & .22 & .20 & .02 & 98.95 \\
\hline $\mathrm{HO}-6$ & $\mathrm{ML}$ & $k 5-1859$ & 1 & ML-57 & a & JMR & 50.56 & 13.03 & 12.19 & nd & 8.46 & 10.24 & 2.04 & .39 & nd & 2.06 & .24 & .16 & nd & 99.37 \\
\hline $\mathrm{HO}-7$ & $\mathrm{HU}$ & hc10 & 1 & PA-2 & d & WSW & 46.96 & 12.94 & 1.42 & 10.31 & 10.40 & 11.42 & 1.91 & .67 & nd & 2.04 & .23 & .18 & nd & 98.48 \\
\hline $\mathrm{HO}-8$ & $\mathrm{HU}$ & h1o & 1 & W-214685 & c & $\mathrm{RBM}$ & 46.00 & 12.80 & 2.90 & 9.12 & 10.50 & 11.50 & 2.30 & .78 & .36 & 2.00 & .27 & .21 & .02 & 98.76 \\
\hline HP-1 & $\mathrm{MK}$ & lc & 1 & D-276070 & b & EWW & 50.00 & 17.40 & 11.20 & nd & 3.39 & 6.69 & 4.56 & 1.98 & .82 & 2.59 & .91 & .22 & nd & 99.76 \\
\hline HP-2 & $\mathrm{ML}$ & $k 2$ & 1 & D-276071 & $b$ & EWW & 49.50 & 12.10 & 11.90 & nd & 12.70 & 9.67 & 1.97 & .34 & .00 & 1.68 & .21 & .16 & nd & 100.23 \\
\hline HP-3 & $\mathrm{ML}$ & kiy & 1 & D-278133 & $b$ & $C D C$ & 50.60 & 13.90 & 12.40 & nd & 7.98 & 10.80 & 2.32 & .44 & .00 & 2.28 & .28 & .17 & nd & 101.17 \\
\hline HP-4 & MK & lc & 1 & D-276068 & b & EWW & 50.20 & 17.10 & 11.30 & nd & 3.96 & 6.88 & 4.83 & 1.96 & .10 & 2.59 & 1.01 & .22 & nd & 100.15 \\
\hline HP-5 & MK & lc & 1 & D-276069 & $\mathrm{b}$ & EWW & 49.40 & 17.10 & 11.90 & nd & 4.05 & 7.05 & 4.63 & 1.90 & .00 & 2.84 & .99 & .21 & nd & 100.07 \\
\hline HP-6 & $\mathrm{ML}$ & k3 & 1 & D-278129 & $b$ & $\mathrm{CDC}$ & 52.10 & 13.80 & 12.40 & nd & 7.14 & 10.70 & 2.17 & .41 & .00 & 2.22 & .28 & .17 & nd & 101.39 \\
\hline HP-7 & ML & $\mathrm{k} 3$ & 1 & D-278130 & b & $\mathrm{CDC}$ & 52.20 & 13.80 & 12.40 & nd & 7.28 & 10.70 & 2.25 & .40 & .00 & 2.19 & .26 & .17 & nd & 101.65 \\
\hline HP-8 & ML & $\mathrm{k} 3$ & 1 & D-278131 & $b$ & $\mathrm{CDC}$ & 51.90 & 13.80 & 12.20 & nd & 7.01 & 10.70 & 2.32 & .39 & .00 & 2.17 & .25 & .17 & nd & 100.91 \\
\hline HP-9 & MK & 1 & 1 & D-260896 & b & WSW & 49.30 & 16.90 & 12.50 & nd & 4.51 & 7.17 & 4.48 & 1.74 & .00 & 3.01 & .85 & .22 & .00 & 100.68 \\
\hline HP-10 & ML & k1y & 1 & D-278132 & b & $\mathrm{CDC}$ & 50.40 & 11.20 & 12.70 & nd & 14.20 & 9.19 & 1.77 & .25 & .00 & 1.75 & .21 & .17 & nd & 101.84 \\
\hline HP-11 & MK & 1 & 1 & D-260899 & $b$ & WSW & 49.90 & 17.10 & 12.00 & nd & 4.09 & 7.05 & 4.68 & 1.85 & .00 & 2.85 & .90 & .22 & .00 & 100.64 \\
\hline HP-12 & ML & $k 1 y$ & 1 & D-278134 & b & $\mathrm{CDC}$ & 52.20 & 13.30 & 12.10 & nd & 8.53 & 10.50 & 2.12 & .33 & .00 & 2.17 & .25 & .17 & nd & 101.67 \\
\hline HP-13 & MK & $\mathrm{hm}$ & 1 & $81-36$ & d & WSW & 45.99 & 13.65 & 14.14 & nd & 8.32 & 10.84 & 2.48 & .82 & .16 & 3.23 & .42 & .20 & nd & 100.25 \\
\hline
\end{tabular}


Table 1.-Chemical analyses-Continued

\begin{tabular}{|c|c|c|c|c|c|c|c|c|c|c|c|c|c|c|c|c|c|c|c|c|}
\hline $\begin{array}{c}\text { Sample } \\
\text { locality } \\
\text { No. }\end{array}$ & $\begin{array}{l}\text { Vol- } \\
\text { cano }\end{array}$ & Unit & $\begin{array}{c}\text { Map } \\
\text { sheet } \\
\text { No. }\end{array}$ & $\begin{array}{l}\text { Laboratory } \\
\text { No. }\end{array}$ & Remark & Collector & $\mathrm{SiO}_{2}$ & $\mathrm{Al}_{2} \mathrm{O}_{3}$ & $\mathrm{Fe}_{2} \mathrm{O}_{3}$ & $\mathrm{FeO}$ & $\mathrm{MgO}$ & $\mathrm{CaO}$ & $\mathrm{Na}_{2} \mathrm{O}$ & $\mathrm{K}_{2} \mathrm{O}$ & $\mathrm{H}_{2} \mathrm{O}$ & $\mathrm{TiO}_{2}$ & $\mathrm{P}_{2} \mathrm{O}_{5}$ & $\mathrm{MnO}$ & $\mathrm{CO}_{2}$ & Total \\
\hline HP-14 & MK & $\mathrm{hm}$ & 1 & $81-46$ & $\bar{d}$ & WSW & 45.36 & 14.81 & 14.11 & $\overline{\text { nd }}$ & 7.45 & 10.55 & 2.49 & .94 & .19 & 3.46 & .46 & .18 & nd & 100.00 \\
\hline HP-15 & MK & 1 & 1 & $81-32$ & d & WSW & 49.82 & 16.97 & 11.72 & nd & 4.04 & 6.88 & 4.32 & 1.84 & .10 & 2.76 & .83 & .22 & nd & 99.50 \\
\hline HP-16 & $\mathrm{HU}$ & hc10 & 1 & KM-7C & d & WSW & 47.51 & 15.22 & 1.46 & 10.72 & 6.91 & 10.72 & 2.68 & .87 & nd & 2.35 & .28 & .18 & nd & 98.90 \\
\hline HP-17 & MK & 1 & 1 & D-260883 & b & WSW & 49.90 & 16.90 & 12.00 & nd & 4.18 & 6.96 & 4.46 & 1.82 & .00 & 2.81 & .88 & .22 & .00 & 100.13 \\
\hline HP-18 & MK & $\mathrm{hm}$ & 1 & KM-9 & d & WSW & 47.75 & 13.64 & 14.95 & nd & 5.31 & 10.00 & 2.72 & .95 & .22 & 3.63 & .46 & .20 & nd & 99.83 \\
\hline HP-19 & MK & $\mathrm{hm}$ & 1 & $81-19$ & d & WSW & 47.48 & 14.21 & 14.15 & nd & 6.42 & 10.99 & 2.41 & .79 & .21 & 3.24 & .40 & .20 & nd & 100.50 \\
\hline HQ-1 & ML & k4 & 1 & D-276064 & b & EWW & 52.00 & 13.80 & 12.00 & nd & 6.80 & 10.80 & 2.25 & .43 & .00 & 2.08 & .26 & .17 & nd & 100.59 \\
\hline $\mathrm{HQ}-2$ & $\mathrm{MK}$ & lc & 1 & D-284764 & b & EWW & 52.40 & 17.60 & 10.30 & nd & 2.97 & 6.12 & 4.74 & 2.24 & .32 & 2.09 & 1.24 & .23 & nd & 100.25 \\
\hline HQ-3 & MK & $\mathrm{hm}$ & 1 & $\mathrm{H}-1$ & d & WSW & $\cdot 43.79$ & 9.57 & 13.77 & nd & 17.84 & 9.91 & 1.20 & .47 & nd & 2.41 & .30 & .19 & nd & 99.45 \\
\hline HQ-4 & MK & lc & 1 & D-276056 & b & EWW & 52.20 & 17.40 & 10.10 & nd & 3.00 & 6.11 & 5.13 & 2.33 & .39 & 2.05 & 1.24 & .23 & nd & 100.18 \\
\hline $\mathrm{HQ}-5$ & MK & $\mathrm{hm}$ & 1 & D-276057 & b & EWW & 46.80 & 14.00 & 15.10 & nd & 5.75 & 10.70 & 2.88 & .71 & .05 & 3.88 & .44 & .20 & nd & 100.51 \\
\hline $\mathrm{HQ}-6$ & MK & lc & 1 & D-276065 & b & EWW & 50.20 & 17.00 & 11.40 & nd & 3.83 & 6.79 & 4.47 & 1.99 & .11 & 2.64 & 1.01 & .21 & nd & 99.65 \\
\hline $\mathrm{HQ}-7$ & MK & $\mathrm{hmc}$ & 1 & D-284765 & b & EWW & 47.30 & 14.20 & 14.60 & nd & 6.19 & 11.20 & 2.69 & .80 & .00 & 3.65 & .48 & .20 & nd & 101.31 \\
\hline $\mathrm{HQ}-8$ & MK & $\mathrm{hm}$ & 1 & D-284774 & b & EWW & 48.00 & 14.40 & 13.70 & nd & 6.67 & 11.40 & 2.56 & .75 & .00 & 3.23 & .38 & .19 & nd & 101.28 \\
\hline HQ-9 & MK & lc & 1 & D-276067 & b & EWW & 49.70 & 17.00 & 11.40 & nd & 3.84 & 6.92 & 4.73 & 1.99 & .00 & 2.66 & 1.01 & .21 & nd & 99.46 \\
\hline $\mathrm{HQ}-10$ & MK & $\mathrm{hmc}$ & 1 & D-284766 & b & EWW & 47.70 & 14.00 & 14.60 & nd & 6.25 & 11.00 & 2.78 & .86 & .00 & 3.64 & .47 & .20 & nd & 101.50 \\
\hline $\mathrm{HQ}-11$ & $\mathrm{MK}$ & IC & 1 & D-276066 & b & EWW & 49.70 & 17.10 & 11.40 & nd & 3.78 & 6.97 & 4.70 & 1.94 & .34 & 2.69 & .99 & .21 & nd & 99.82 \\
\hline HQ-12 & MK & 1 & 1 & D-292989 & b & WSW & 49.80 & 17.20 & 11.50 & nd & 3.86 & 6.95 & 4.67 & 1.96 & .19 & 2.74 & 1.02 & .21 & nd & 100.10 \\
\hline $\mathrm{HQ}-15$ & MK & $\mathrm{hm}$ & 1 & D-284771 & b & EWW & 48.00 & 13.90 & 14.80 & nd & 5.81 & 10.70 & 2.76 & .83 & .00 & 3.73 & .44 & .20 & nd & 101.17 \\
\hline $\mathrm{HQ}-16$ & MK & $\mathrm{hm}$ & 1 & D-284770 & b & EWW & 48.70 & 13.30 & 13.60 & nd & 7.29 & 11.60 & 2.43 & .67 & .00 & 3.40 & .35 & .18 & nd & 101.52 \\
\hline $\mathrm{HQ}-17$ & MK & $\mathrm{hm}$ & 1 & D-284767 & b & EWW & 48.10 & 14.00 & 14.70 & nd & 5.64 & 10.50 & 2.94 & .98 & .00 & 3.73 & .45 & .20 & nd & 101.24 \\
\hline $\mathrm{HQ}-18$ & MK & $\mathrm{hm}$ & 1 & D-284768 & b & EWW & 48.40 & 13.30 & 13.40 & nd & 8.05 & 11.60 & 2.26 & .63 & .00 & 2.95 & .35 & .18 & nd & 101.12 \\
\hline $\mathrm{HQ}-19$ & MK & $\mathrm{hm}$ & 1 & D-284769 & b & EWW & 48.80 & 13.50 & 13.40 & nd & 6.97 & 11.90 & 2.41 & .69 & .00 & 2.99 & .36 & .19 & nd & 101.21 \\
\hline HQ-20 & MK & I & 1 & D-292990 & b & WSW & 50.70 & 17.30 & 11.30 & nd & 3.62 & 6.74 & 4.61 & 2.04 & .00 & 2.43 & 1.11 & .21 & nd & 100.06 \\
\hline $\mathrm{HQ}-22$ & MK & I & 1 & D-292986 & b & WSW & 50.20 & 17.10 & 11.10 & nd & 3.59 & 6.79 & 4.91 & 2.02 & .43 & 2.51 & 1.11 & .22 & nd & 99.98 \\
\hline $\mathrm{HQ}-23$ & MK & 1 & 1 & D-292994 & b & WSW & 52.80 & 17.30 & 9.47 & nd & 3.11 & 5.98 & 5.20 & 2.36 & .23 & 1.94 & 1.01 & .21 & nd & 99.61 \\
\hline $\mathrm{HQ}-24$ & MK & $\mathrm{hm}$ & 1 & D-292947 & $b$ & WSW & 47.00 & 14.10 & 14.60 & nd & 5.85 & 10.80 & 2.96 & 1.04 & .00 & 3.94 & .53 & .19 & nd & 101.01 \\
\hline $\mathrm{HQ}-25$ & $\mathrm{MK}$ & $\mathrm{hm}$ & 1 & D-292948 & b & WSW & 47.10 & 14.20 & 14.60 & nd & 5.81 & 10.80 & 3.00 & 1.02 & .00 & 3.96 & .53 & 19 & nd & 101.21 \\
\hline HQ-26 & $\mathrm{MK}$ & $\mathrm{hm}$ & 1 & D-292949 & $\mathrm{b}$ & WSW & 47.00 & 14.50 & 14.80 & nd & 5.99 & 11.20 & 2.57 & .68 & .00 & 3.47 & .43 & .20 & nd & 100.84 \\
\hline $\mathrm{HQ}-27$ & $\mathrm{MK}$ & $\mathrm{hm}$ & 1 & D-292943 & b & WSW & 47.70 & 14.30 & 14.70 & nd & 5.85 & 11.20 & 2.66 & .83 & .00 & 3.39 & .42 & .20 & nd & 101.25 \\
\hline $\mathrm{HQ}-28$ & MK & I & 1 & D-292993 & b & WSW & 51.00 & 17.40 & 11.00 & nd & 3.47 & 6.83 & 4.94 & 2.10 & .00 & 2.38 & 1.15 & .21 & nd & 100.48 \\
\hline $\mathrm{HQ}-29$ & MK & 1 & 1 & D-292945 & b & WSW & 50.70 & 16.90 & 10.90 & nd & 3.55 & 6.63 & 5.01 & 2.11 & .00 & 2.40 & 1.12 & .22 & nd & 99.54 \\
\hline $\mathrm{HQ}-30$ & MK & 1 & 1 & D-292946 & b & WSW & 51.20 & 17.20 & 10.90 & nd & 3.50 & 6.67 & 5.02 & 2.12 & .00 & 2.43 & 1.14 & .23 & nd & 100.41 \\
\hline $\mathrm{HQ}-31$ & MK & 1 & 1 & D-292991 & $\mathrm{b}$ & WSW & 50.50 & 17.10 & 11.40 & nd & 4.01 & 6.88 & 4.48 & 1.98 & .00 & 2.65 & .95 & .22 & nd & 100.17 \\
\hline $\mathrm{HQ}-32$ & MK & 1 & 1 & D-292961 & $\mathrm{b}$ & WSW & 51.90 & 17.30 & 10.40 & nd & 3.33 & 6.56 & 4.94 & 2.18 & .00 & 2.32 & 1.16 & .23 & nd & 100.32 \\
\hline $\mathrm{HQ}-33$ & MK & 1 & 1 & D-292957 & b & WSW & 50.60 & 17.00 & 11.30 & nd & 3.87 & 6.88 & 4.78 & 1.99 & .00 & 2.63 & .97 & .22 & nd & 100.24 \\
\hline HQ-34 & MK & 1 & 1 & D-292960 & b & WSW & 51.60 & 17.20 & 10.50 & nd & 3.67 & 6.51 & 4.77 & 2.16 & .00 & 2.38 & .99 & .21 & nd & 99.99 \\
\hline $\mathrm{HQ}-35$ & MK & I & 1 & D-292966 & b & WSW & 52.60 & 17.60 & 9.70 & nd & 3.04 & 5.94 & 5.06 & 2.37 & .02 & 2.14 & 1.06 & .22 & nd & 99.75 \\
\hline HQ-36 & MK & 1 & 1 & D-292958 & $b$ & WSW & 51.00 & 17.10 & 11.10 & nd & 3.72 & 6.67 & 4.74 & 2.05 & .00 & 2.53 & .98 & .22 & nd & 100.11 \\
\hline $\mathrm{HQ}-37$ & MK & i & 1 & D-292983 & b & WSW & 52.90 & 17.30 & 9.49 & nd & 3.05 & 6.16 & 5.03 & 2.34 & .14 & 2.08 & 1.05 & .22 & nd & 99.76 \\
\hline
\end{tabular}


Table 1.-Chemical analyses-Continued

\begin{tabular}{|c|c|c|c|c|c|c|c|c|c|c|c|c|c|c|c|c|c|c|c|c|}
\hline $\begin{array}{c}\text { Sample } \\
\text { locality } \\
\text { No. }\end{array}$ & $\begin{array}{l}\text { Vol- } \\
\text { cano }\end{array}$ & Unit & $\begin{array}{c}\text { Map } \\
\text { sheet } \\
\text { No. }\end{array}$ & $\begin{array}{l}\text { Laboratory } \\
\text { No. }\end{array}$ & Remark & Collector & $\mathrm{SiO}_{2}$ & $\mathrm{Al}_{2} \mathrm{O}_{3}$ & $\mathrm{Fe}_{2} \mathrm{O}_{3}$ & $\mathrm{FeO}$ & $\mathrm{MgO}$ & $\mathrm{CaO}$ & $\mathrm{Na}_{2} \mathrm{O}$ & $\mathrm{K}_{2} \mathrm{O}$ & $\mathrm{H}_{2} \mathrm{O}$ & $\mathrm{TiO}_{2}$ & $\mathrm{P}_{2} \mathrm{O}_{5}$ & $\mathrm{MnO}$ & $\mathrm{CO}_{2}$ & Total \\
\hline HQ-38 & $\mathrm{MK}$ & 1 & 1 & D-292956 & $\mathrm{b}$ & WSW & 50.40 & 17.20 & 11.40 & nd & 3.92 & 7.00 & 4.58 & 1.95 & .00 & 2.66 & .98 & .22 & nd & 100.31 \\
\hline HQ-39 & MK & 1 & 1 & D-292959 & b & WSW & 51.60 & 17.40 & 10.40 & nd & 3.50 & 6.55 & 4.82 & 2.17 & .00 & 2.38 & .98 & .21 & nd & 100.01 \\
\hline HQ-40 & MK & 1 & 1 & D-292955 & $b$ & WSW & 50.90 & 17.20 & 11.00 & nd & 3.87 & 6.94 & 4.58 & 2.02 & .00 & 2.59 & .97 & .22 & nd & 100.29 \\
\hline HQ-41 & MK & i & 1 & D-292984 & $b$ & WSW & 50.80 & 17.20 & 11.10 & nd & 3.62 & 6.77 & 4.85 & 2.05 & .00 & 2.49 & 1.11 & .22 & nd & 100.21 \\
\hline $\mathrm{HQ}-42$ & MK & lc & 1 & D-292967 & b & WSW & 51.00 & 17.10 & 10.90 & nd & 3.55 & 6.70 & 4.97 & 2.11 & .00 & 2.46 & 1.11 & .22 & nd & 100.12 \\
\hline HQ-43 & MK & 1 & 1 & D-284763 & b & EWW & 50.70 & 17.20 & 11.40 & nd & 3.59 & 6.92 & 4.65 & 2.06 & .00 & 2.52 & 1.12 & .22 & nd & 100.38 \\
\hline HQ-44 & MK & $i$ & 1 & D-292965 & $b$ & WSW & 51.30 & 17.40 & 10.70 & nd & 3.30 & 6.53 & 4.94 & 2.15 & .24 & 2.27 & 1.26 & .22 & nd & 100.31 \\
\hline HQ-45 & MK & 1 & 1 & D-292964 & b & WSW & 51.50 & 17.30 & 10.50 & nd & 3.41 & 6.77 & 4.72 & 2.11 & .21 & 2.30 & 1.13 & .22 & nd & 100.17 \\
\hline HQ-46 & $\mathrm{MK}$ & 1 & 1 & D-292968 & b & WSW & 50.10 & 17.40 & 11.20 & nd & 3.73 & 6.72 & 4.63 & 1.96 & .57 & 2.59 & 1.05 & .22 & nd & 100.17 \\
\hline $\mathrm{HQ}-47$ & MK & 1 & 1 & D-292962 & $\mathrm{b}$ & WSW & 50.80 & 17.10 & 11.10 & nd & 3.69 & 6.81 & 4.85 & 2.03 & .00 & 2.52 & 1.04 & .22 & nd & 100.16 \\
\hline HQ-48 & MK & hme & 1 & D-292992 & b & WSW & 46.00 & 14.80 & 14.30 & nd & 5.88 & 11.10 & 2.48 & .71 & .94 & 3.38 & .42 & .19 & nd & 100.20 \\
\hline$H Q-49$ & MK & lc & 1 & D-292963 & b & WSW & 50.70 & 16.80 & 10.90 & nd & 3.68 & 6.90 & 4.56 & 1.92 & .89 & 2.49 & 1.03 & .22 & nd & 100.09 \\
\hline HQ-50 & MK & 1 & 1 & D-292985 & b & WSW & 50.20 & 17.20 & 11.40 & nd & 3.55 & 6.82 & 4.63 & 2.03 & .24 & 2.52 & 1.14 & .22 & nd & 99.95 \\
\hline $\mathrm{HQ}-51$ & MK & 1 & 1 & D-278155 & b & EWW & 49.80 & 17.00 & 11.80 & nd & 4.06 & 7.10 & 4.67 & 1.91 & .00 & 2.82 & .95 & .21 & nd & 100.32 \\
\hline HQ-52 & MK & 1 & 1 & D-292976 & b & WSW & 49.80 & 17.00 & 11.80 & nd & 4.33 & 7.01 & 4.55 & 1.82 & .01 & 2.72 & .91 & .22 & nd & 100.17 \\
\hline HQ-53 & MK & 1 & 1 & D-292977 & b & WSW & 50.20 & 16.90 & 11.40 & nd & 4.10 & 6.90 & 4.77 & 1.99 & .00 & 2.57 & 1.04 & .22 & nd & 100.09 \\
\hline HQ-54 & MK & 1 & 1 & D-292979 & b & WSW & 49.70 & 16.80 & 11.70 & nd & 4.49 & 7.02 & 4.50 & 1.84 & .00 & 2.68 & .90 & .22 & nd & 99.85 \\
\hline HQ-55 & MK & 1 & 1 & D-292971 & b & WSW & 50.30 & 17.10 & 11.50 & nd & 3.96 & 6.89 & 4.66 & 1.94 & .00 & 2.63 & .92 & .22 & nd & 100.12 \\
\hline HQ-56 & MK & 1 & 1 & D-292982 & b & WSW & 48.80 & 17.20 & 11.80 & nd & 4.07 & 7.03 & 4.50 & 1.79 & .90 & 2.78 & .98 & .22 & nd & 100.07 \\
\hline $\mathrm{HQ}-57$ & MK & 1 & 1 & D-292978 & b & WSW & 50.10 & 16.80 & 11.50 & nd & 4.63 & 6.86 & 4.72 & 1.95 & .00 & 2.56 & 1.02 & .22 & nd & 100.36 \\
\hline HQ-58 & MK & 1 & 1 & D-292975 & b & WSW & 51.30 & 17.10 & 10.90 & nd & 3.55 & 6.44 & 4.83 & 2.11 & .00 & 2.36 & 1.01 & .22 & nd & 99.82 \\
\hline HQ-59 & MK & 1 & 1 & D-292972 & b & WSW & 50.60 & 17.20 & 11.40 & nd & 3.88 & 6.81 & 4.64 & 1.98 & .00 & 2.61 & .93 & .22 & nd & 100.27 \\
\hline$H Q-60$ & MK & 1 & 1 & D-292973 & b & WSW & 50.50 & 16.90 & 11.30 & nd & 3.72 & 6.68 & 4.68 & 1.98 & .32 & 2.54 & .94 & .22 & nd & 99.78 \\
\hline$H Q-61$ & MK & I & 1 & D-292974 & b & WSW & 50.10 & 16.60 & 11.90 & nd & 5.20 & 7.04 & 4.32 & 1.82 & .00 & 2.61 & 85 & .22 & nd & 100.66 \\
\hline HQ-62 & MK & I & 1 & D-292969 & b & WSW & 49.40 & 17.00 & 11.60 & nd & 3.94 & 6.75 & 4.60 & 1.89 & .35 & 2.67 & .93 & .21 & nd & 99.34 \\
\hline HQ-63 & MK & 1 & 1 & D-278154 & b & EWW & 50.30 & 17.00 & 11.90 & nd & 4.18 & 7.03 & 4.65 & 1.83 & .00 & 2.79 & .90 & .22 & nd & 100.80 \\
\hline HQ-64 & MK & 1 & 1 & D-292970 & b & WSW & 50.10 & 17.00 & 11.60 & nd & 3.99 & 6.83 & 4.40 & 1.94 & .25 & 2.72 & .93 & .22 & nd & 99.98 \\
\hline HQ-65 & MK & 1 & 1 & D-292980 & b & Wsw & 49.90 & 17.00 & 11.90 & nd & 4.22 & 7.13 & 4.66 & 1.86 & .00 & 2.84 & .89 & .22 & nd & 100.62 \\
\hline HQ-66 & MK & 1 & 1 & D-292981 & b & WSW & 49.80 & 16.90 & 11.90 & nd & 4.22 & 7.10 & 4.60 & 1.87 & .00 & 2.83 & .89 & .22 & nd & 100.33 \\
\hline $\mathrm{HQ}-67$ & MK & 1 & 1 & D-292987 & b & WSW & 49.60 & 17.10 & 11.70 & nd & 4.14 & 7.20 & 4.53 & 1.88 & .00 & 2.76 & .97 & .21 & nd & 100.09 \\
\hline$H Q-68$ & MK & 1 & 1 & D-292988 & b & WSW & 50.50 & 17.00 & 11.30 & nd & 3.96 & 6.68 & 4.71 & 1.97 & .08 & 2.64 & .88 & .22 & nd & 99.94 \\
\hline HR-1 & MK & lc & 1 & D-284758 & b & EWW & 50.10 & 17.00 & 11.80 & nd & 4.06 & 6.99 & 4.60 & 1.89 & .00 & 2.76 & .91 & .23 & nd & 100.34 \\
\hline HR-2 & MK & 1 & 1 & D-278094 & b & EWW & 50.40 & 17.10 & 11.30 & nd & 3.93 & 6.91 & 4.55 & 1.95 & .00 & 2.71 & .88 & .21 & nd & 99.94 \\
\hline HR-3 & MK & 1 & 1 & D-276054 & b & EWW & 51.00 & 17.40 & 10.80 & nd & 3.68 & 6.72 & 4.83 & 2.08 & .21 & 2.53 & 1.03 & .22 & nd & 100.50 \\
\hline $\mathrm{HR}-4$ & MK & lc & 1 & D-278093 & b & EWW & 50.20 & 16.90 & 11.10 & nd & 3.74 & 6.80 & 4.55 & 1.97 & .00 & 2.58 & .96 & .22 & nd & 99.02 \\
\hline $\mathrm{HR}-6$ & MK & lc & 1 & D-284756 & b & EWW & 49.80 & 17.10 & 11.70 & nd & 4.09 & 7.21 & 4.45 & 1.87 & .02 & 2.84 & .93 & .22 & nd & 100.23 \\
\hline HR-7 & MK & I & 1 & D-276051 & b & EWW & 51.40 & 17.30 & 10.90 & nd & 3.44 & 6.58 & 4.99 & 2.11 & .00 & 2.30 & 1.15 & .22 & nd & 100.39 \\
\hline HR-8 & MK & $\mathrm{hm}$ & 1 & D-284759 & b & EWW & 48.30 & 14.00 & 13.90 & nd & 7.02 & 11.80 & 2.31 & .68 & .00 & 2.92 & .36 & .19 & nd & 101.48 \\
\hline HR-9 & MK & $\mathrm{hm}$ & 1 & D-284750 & b & EWW & 48.30 & 12.90 & 13.60 & nd & 7.47 & 12.30 & 2.21 & .59 & .25 & 2.89 & .34 & .19 & nd & 101.04 \\
\hline HR-11 & MK & ly & 1 & D-284748 & b & EWW & 51.40 & 17.20 & 11.00 & nd & 3.74 & 6.66 & 4.78 & 2.04 & .00 & 2.56 & .89 & .21 & nd & 100.48 \\
\hline
\end{tabular}


Table 1.-Chemical analyses-Continued

\begin{tabular}{|c|c|c|c|c|c|c|c|c|c|c|c|c|c|c|c|c|c|c|c|c|}
\hline $\begin{array}{c}\text { Sample } \\
\text { locality } \\
\text { No. }\end{array}$ & $\begin{array}{l}\text { Vol- } \\
\text { cano }\end{array}$ & Unit & $\begin{array}{l}\text { Map } \\
\text { sheet } \\
\text { No. }\end{array}$ & $\begin{array}{l}\text { Laboratory } \\
\text { No. }\end{array}$ & Remark & Collector & $\mathrm{SiO}_{2}$ & $\mathrm{Al}_{2} \mathrm{O}_{3}$ & $\mathrm{Fe}_{2} \mathrm{O}_{3}$ & $\mathrm{FeO}$ & $\mathrm{MgO}$ & $\mathrm{CaO}$ & $\mathrm{Na}_{2} \mathrm{O}$ & $\mathrm{K}_{2} \mathrm{O}$ & $\mathrm{H}_{2} \mathrm{O}$ & $\mathrm{TiO}_{2}$ & $\mathrm{P}_{2} \mathrm{O}_{5}$ & $\mathrm{MnO}$ & $\mathrm{CO}_{2}$ & Total \\
\hline HR-14 & MK & lc & 1 & D-278118 & $\mathrm{b}$ & EWW & 51.50 & 17.10 & 10.80 & nd & 3.49 & 6.52 & 5.01 & 2.13 & .14 & 2.25 & 1.15 & .22 & nd & 100.31 \\
\hline HR-15 & MK & Iy & 1 & D-284755 & $\mathrm{b}$ & EWW & 53.40 & 17.20 & 10.10 & nd & 2.84 & 5.90 & 4.94 & 2.33 & .00 & 1.99 & 1.16 & .24 & nd & 100.10 \\
\hline HR-17 & MK & $\mathrm{hm}$ & 1 & D-284754 & $\mathrm{b}$ & EWW & 47.00 & 14.00 & 15.50 & nd & 5.60 & 10.70 & 2.67 & .69 & .00 & 3.81 & .48 & .21 & nd & 100.66 \\
\hline HR-18 & MK & 1 & 1 & D-278081 & b & EWW & 50.50 & 17.10 & 11.30 & nd & 3.77 & 6.85 & 4.84 & 1.99 & .00 & 2.61 & 1.07 & .22 & nd & 100.25 \\
\hline HR-19 & MK & 1 & 1 & D-278082 & $\mathrm{b}$ & EWW & 51.10 & 17.20 & 10.80 & nd & 3.68 & 6.51 & 4.87 & 2.11 & .02 & 2.55 & .93 & .21 & nd & 99.98 \\
\hline HR-20 & MK & I & 1 & D-276053 & $\mathrm{b}$ & EWW & 50.20 & 17.50 & 11.00 & nd & 3.61 & 6.77 & 4.74 & 1.95 & .55 & 2.53 & 1.09 & .23 & nd & 100.17 \\
\hline HR-22 & MK & 1 & 1 & D-276055 & $b$ & EWW & 50.80 & 17.30 & 11.00 & nd & 3.70 & 6.84 & 5.01 & 2.06 & .00 & 2.56 & 1.07 & .22 & nd & 100.56 \\
\hline HR-23 & MK & lc & 1 & D-284747 & $\mathrm{b}$ & EWW & 50.40 & 17.00 & 11.80 & nd & 4.06 & 7.08 & 4.57 & 1.87 & .00 & 2.79 & .92 & .22 & nd & 100.71 \\
\hline HR-25 & MK & lc & 1 & D-278080 & $\mathrm{b}$ & EWW & 50.60 & 17.20 & 10.70 & nd & 3.67 & 6.73 & 4.86 & 2.04 & .35 & 2.48 & .99 & .21 & nd & 99.83 \\
\hline HR-26 & MK & lc & 1 & D-276052 & $\mathrm{b}$ & EWW & 51.00 & 17.30 & 10.80 & nd & 3.37 & 6.37 & 5.11 & 2.13 & .38 & 2.26 & 1.16 & .22 & nd & 100.10 \\
\hline HR-27 & MK & lc & 1 & D-278119 & $\mathrm{b}$ & EWW & 50.90 & 17.10 & 11.20 & nd & 3.56 & 6.76 & 4.86 & 2.05 & .00 & 2.45 & 1.13 & .22 & nd & 100.23 \\
\hline HR-28 & MK & 1 & 1 & D-284746 & $b$ & EWW & 50.10 & 16.90 & 11.80 & nd & 4.14 & 7.06 & 4.61 & 1.83 & .00 & 2.78 & .90 & .22 & nd & 100.34 \\
\hline HR-29 & MK & ly & 1 & D-284739 & $b$ & EWW & 52.20 & 17.20 & 10.80 & nd & 3.36 & 6.36 & 4.95 & 2.11 & .00 & 2.24 & 1.08 & .23 & nd & 100.53 \\
\hline HR-30 & MK & I & 1 & D-284745 & b & EWW & 50.20 & 17.00 & 11.80 & nd & 4.11 & 7.07 & 4.55 & 1.89 & .00 & 2.84 & .89 & .22 & nd & 100.57 \\
\hline HR-31 & MK & $\mathrm{hm}$ & 1 & D-284742 & $b$ & EWW & 47.70 & 13.80 & 15.30 & nd & 5.53 & 10.50 & 2.91 & .87 & .00 & 3.93 & .49 & .21 & nd & 101.24 \\
\hline HR-32 & MK & 1 & 1 & D-278083 & b & EWW & 51.50 & 17.20 & 10.80 & nd & 3.38 & 6.49 & 5.04 & 2.16 & .00 & 2.25 & 1.19 & .23 & nd & 100.24 \\
\hline HR-33 & MK & 1 & 1 & D-278117 & b & EWW & 50.60 & 17.10 & 11.20 & nd & 3.85 & 6.98 & 4.89 & 2.00 & .00 & 2.68 & 1.02 & .22 & nd & 100.54 \\
\hline HR-34 & MK & $\mathrm{hm}$ & 1 & D-284741 & b & EWW & 47.90 & 14.10 & 15.00 & nd & 5.46 & 10.70 & 2.90 & .91 & .00 & 3.76 & .49 & .21 & nd & 101.43 \\
\hline HR-35 & MK & $\mathrm{hm}$ & 1 & D-284740 & b & EWW & 48.20 & 13.80 & 15.10 & nd & 5.65 & 10.70 & 2.93 & .87 & .00 & 3.71 & .47 & .21 & nd & 101.64 \\
\hline HR-36 & MK & lc & 1 & D-278144 & b & EWW & 50.40 & 17.00 & 11.40 & nd & 3.93 & 7.02 & 4.54 & 1.91 & .43 & 2.66 & .96 & .22 & nd & 100.47 \\
\hline HR-37 & MK & I & 1 & D-278116 & b & EWW & 49.60 & 17.20 & 11.10 & nd & 3.79 & 6.93 & 4.35 & 1.95 & .95 & 2.68 & 1.02 & .22 & nd & 99.79 \\
\hline HR-40 & MK & lc & 1 & D-278135 & b & EWW & 51.40 & 17.00 & 11.00 & nd & 3.36 & 6.29 & 5.09 & 2.20 & .00 & 2.21 & 1.34 & .23 & nd & 100.12 \\
\hline HR-41 & MK & $\mathrm{hm}$ & 1 & D-278145 & $\mathrm{b}$ & EWW & 48.70 & 13.50 & 15.40 & nd & 5.17 & 9.87 & 3.06 & 1.10 & .00 & 4.08 & .54 & .21 & nd & 101.63 \\
\hline HR-42 & MK & $\mathrm{hm}$ & 1 & D-278146 & b & EWW & 48.30 & 13.90 & 14.20 & nd & 6.69 & 12.00 & 2.22 & .60 & .00 & 3.12 & .36 & .19 & nd & 101.58 \\
\hline HR-43 & MK & $\mathrm{hmc}$ & 1 & D-278111 & b & EWW & 47.00 & 16.30 & 11.40 & nd & 7.09 & 12.40 & 2.25 & .40 & .72 & 2.73 & .31 & .16 & nd & 100.76 \\
\hline HR-44 & MK & $\mathrm{hm}$ & 1 & D-292954 & b & EWW & 47.60 & 10.60 & 13.10 & nd & 14.00 & 10.80 & 1.84 & .43 & .00 & 2.32 & .26 & .18 & nd & 101.13 \\
\hline HR-45 & MK & 1 & 1 & D-284743 & b & EWW & 50.60 & 17.20 & 11.20 & nd & 3.89 & 6.94 & 4.86 & 1.97 & .11 & 2.61 & 1.00 & .22 & nd & 100.60 \\
\hline HR-46 & MK & $\mathrm{hm}$ & 1 & $65-1$ & d & WSW & 46.38 & 8.94 & 13.34 & nd & 17.93 & 9.65 & 1.43 & .35 & .17 & 1.86 & .23 & .18 & nd & 100.46 \\
\hline HR-47 & MK & $\mathrm{hm}$ & 1 & D-284737 & b & EWW & 48.30 & 12.70 & 13.20 & nd & 8.86 & 11.70 & 2.17 & .57 & .08 & 2.79 & .33 & .18 & nd & 100.88 \\
\hline HR-50 & MK & 1 & 1 & D-284762 & b & EWW & 51.10 & 17.20 & 11.10 & nd & 3.60 & 6.70 & 5.04 & 2.09 & .00 & 2.42 & 1.10 & .22 & nd & 100.57 \\
\hline HR-51 & MK & $\mathrm{hm}$ & 1 & D-284734 & b & EWW & 47.80 & 14.80 & 13.90 & nd & 6.31 & 12.20 & 2.48 & .62 & .00 & 2.96 & .35 & .19 & nd & 101.61 \\
\hline HR-52 & MK & $\mathrm{hm}$ & 1 & D-284733 & b & EWW & 47.60 & 14.20 & 13.80 & nd & 6.59 & 12.00 & 2.46 & .69 & .00 & 3.15 & .38 & .19 & nd & 101.06 \\
\hline HR-55 & MK & 1 & 1 & D-278112 & b & EWW & 49.10 & 16.80 & 12.30 & nd & 4.52 & 7.34 & 4.47 & 1.75 & .00 & 3.06 & .86 & .21 & nd & 100.41 \\
\hline HR-56 & MK & $\mathrm{hm}$ & 1 & D-284738 & b & EWW & 48.60 & 13.60 & 15.50 & nd & 5.07 & 9.85 & 3.10 & 1.07 & .00 & 4.02 & .56 & .21 & nd & 101.58 \\
\hline HR-57 & MK & $\mathrm{hm}$ & 1 & D-292953 & b & EWW & 48.60 & 13.10 & 12.90 & nd & 7.84 & 12.00 & 2.23 & .59 & .06 & 2.87 & .33 & .18 & nd & 100.70 \\
\hline HR-58 & MK & 1 & 1 & D-284744 & b & EWW & 51.00 & 17.00 & 10.90 & nd & 3.34 & 6.77 & 5.06 & 2.15 & .00 & 2.41 & 1.35 & .22 & nd & 100.20 \\
\hline HR-59 & MK & $\mathrm{hm}$ & 1 & D-278113 & $\mathrm{b}$ & EWW & 46.30 & 13.50 & 14.00 & nd & 9.04 & 11.40 & 2.10 & .26 & 1.11 & 3.03 & .36 & .19 & nd & 101.29 \\
\hline HR-60 & MK & lc & 1 & D-284760 & b & EWW & 49.90 & 16.90 & 11.10 & nd & 3.67 & 6.44 & 4.27 & 2.01 & 1.79 & 2.35 & 1.12 & .21 & nd & 99.76 \\
\hline HR-61 & MK & $\mathrm{hm}$ & 1 & D-278114 & b & EWW & 47.70 & 13.70 & 13.50 & nd & 7.86 & 10.50 & 2.62 & .74 & .19 & 3.11 & .42 & .19 & nd & 100.53 \\
\hline HR-62 & MK & $\mathrm{hm}$ & 1 & D-278115 & b & EWW & 50.10 & 14.00 & 13.80 & nd & 3.88 & 8.06 & 3.96 & 1.49 & .00 & 3.45 & 1.28 & .22 & nd & 100.24 \\
\hline
\end{tabular}


Table 1.-Chemical analyses-Continued

\begin{tabular}{|c|c|c|c|c|c|c|c|c|c|c|c|c|c|c|c|c|c|c|c|c|}
\hline $\begin{array}{c}\text { Sample } \\
\text { locality } \\
\text { No. }\end{array}$ & $\begin{array}{l}\text { Vol- } \\
\text { cano }\end{array}$ & Unit & $\begin{array}{c}\text { Map } \\
\text { sheet } \\
\text { No. }\end{array}$ & $\begin{array}{c}\text { Laboratory } \\
\text { No. }\end{array}$ & Remark & Collector & $\mathrm{SiO}_{2}$ & $\mathrm{Al}_{2} \mathrm{O}_{3}$ & $\mathrm{Fe}_{2} \mathrm{O}_{3}$ & $\mathrm{FeO}$ & $\mathrm{MgO}$ & $\mathrm{CaO}$ & $\mathrm{Na}_{2} \mathrm{O}$ & $\mathrm{K}_{2} \mathrm{O}$ & $\mathrm{H}_{2} \mathrm{O}$ & $\mathrm{TiO}_{2}$ & $\mathrm{P}_{2} \mathrm{O}_{5}$ & $\mathrm{MnO}$ & $\mathrm{CO}_{2}$ & Total \\
\hline HR-63 & MK & 1 & 1 & D-278142 & $\bar{b}$ & EWW & 50.00 & 17.00 & 11.90 & nd & 4.23 & 7.20 & 4.70 & 1.83 & .00 & 2.87 & .89 & .21 & nd & 100.83 \\
\hline HR-64 & MK & c & 1 & D-278141 & b & EWW & 49.80 & 16.80 & 11.00 & nd & 3.86 & 6.41 & 4.35 & 2.15 & 1.80 & 2.40 & 1.10 & .21 & nd & 99.88 \\
\hline HR-65 & MK & I & 1 & D-278110 & $b$ & EWW & 48.30 & 16.80 & 12.70 & nd & 4.94 & 7.41 & 4.35 & 1.68 & .00 & 3.21 & .82 & .21 & nd & 100.42 \\
\hline HR-66 & MK & 1 & 1 & D-284761 & b & EWW & 50.90 & 17.10 & 11.00 & nd & 3.40 & 6.66 & 5.02 & 2.11 & .07 & 2.35 & 1.22 & .23 & nd & 100.06 \\
\hline HR-67 & MK & 1 & 1 & D-278108 & b & EWW & 48.20 & 16.80 & 12.50 & nd & 4.95 & 7.56 & 4.15 & 1.63 & .00 & 3.19 & .80 & .20 & nd & 99.98 \\
\hline HR-68 & MK & $i$ & 1 & D-278143 & b & EWW & 49.70 & 16.80 & 11.60 & nd & 4.33 & 7.50 & 4.53 & 1.81 & .29 & 2.90 & .87 & .21 & nd & 100.54 \\
\hline HR-69 & MK & $i$ & 1 & D-278140 & b & EWW & 50.40 & 17.00 & 11.60 & nd & 4.14 & 7.10 & 4.70 & 1.88 & .00 & 2.83 & .88 & .21 & nd & 100.74 \\
\hline HR-70 & MK & 1 & 1 & D-278109 & b & EWW & 48.10 & 16.80 & 12.60 & nd & 4.83 & 7.44 & 4.30 & 1.70 & .00 & 3.19 & .83 & .21 & nd & 100.00 \\
\hline HR-71 & MK & 1 & 1 & D-284732 & $b$ & EWW & 50.70 & 17.20 & 11.10 & nd & 3.69 & 7.05 & 4.75 & 2.06 & .00 & 2.56 & 1.08 & .22 & nd & 100.41 \\
\hline HR-72 & MK & 1 & 1 & D-278139 & b & EWW & 51.20 & 17.00 & 10.90 & nd & 3.55 & 6.67 & 4.86 & 2.04 & .14 & 2.47 & 1.01 & .22 & nd & 100.06 \\
\hline HR-73 & MK & 1 & 1 & D-278138 & b & EWW & 50.40 & 17.20 & 11.60 & nd & 4.13 & 7.17 & 4.51 & 1.91 & .00 & 2.86 & .91 & .21 & nd & 100.90 \\
\hline HR-74 & MK & lc & 1 & D-278107 & b & EWW & 49.00 & 17.00 & 12.10 & nd & 4.29 & 7.20 & 4.37 & 1.77 & 1.29 & 2.97 & .88 & .22 & nd & 101.09 \\
\hline HR-75 & MK & I & 1 & D-278095 & b & EWW & 50.20 & 17.00 & 11.60 & nd & 4.03 & 6.98 & 4.80 & 1.93 & .00 & 2.75 & .95 & .22 & nd & 100.46 \\
\hline HR-76 & MK & i & 1 & D-278137 & b & EWW & 50.60 & 17.00 & 11.50 & nd & 4.01 & 6.97 & 4.68 & 1.94 & .00 & 2.70 & .95 & .22 & nd & 100.57 \\
\hline HR-77 & MK & lc & 1 & D-278098 & b & EWW & 50.70 & 17.00 & 11.20 & nd & 3.80 & 6.79 & 4.81 & 1.99 & .00 & 2.58 & .98 & .22 & nd & 100.07 \\
\hline HR-78 & MK & 1 & 1 & D-278136 & b & EWW & 51.30 & 17.10 & 10.70 & nd & 3.51 & 6.60 & 4.68 & 2.14 & .41 & 2.32 & 1.17 & .22 & nd & 100.15 \\
\hline HR-79 & MK & I & 1 & D-278097 & $\mathrm{b}$ & EWW & 49.50 & 16.90 & 11.60 & nd & 4.22 & 7.22 & 4.63 & 1.84 & .00 & 2.86 & .95 & .21 & nd & 99.93 \\
\hline HR-80 & MK & i & 1 & D-278096 & $b$ & EWW & 51.10 & 17.30 & 10.90 & nd & 3.71 & 6.73 & 4.99 & 2.07 & .00 & 2.55 & .98 & .21 & nd & 100.54 \\
\hline HR-81 & $\mathrm{MK}$ & I & 1 & D-278099 & b & EWW & 50.90 & 16.90 & 11.10 & nd & 3.69 & 6.77 & 4.87 & 2.01 & .00 & 2.56 & .99 & .21 & nd & 100.00 \\
\hline HR-82 & MK & lc & 1 & D-278106 & b & EWW & 49.80 & 17.10 & 11.40 & nd & 3.81 & 6.79 & 4.27 & 1.93 & 1.90 & 2.65 & .99 & .22 & nd & 100.86 \\
\hline HR-84 & MK & 1 & 1 & D-278084 & b & EWW & 49.60 & 16.90 & 11.70 & nd & 4.04 & 7.03 & 4.50 & 1.78 & .06 & 2.78 & .92 & .22 & nd & 99.53 \\
\hline HR-85 & MK & 1 & 1 & D-278100 & b & EWW & 50.30 & 17.10 & 11.20 & nd & 3.75 & 7.01 & 4.48 & 1.94 & .51 & 2.58 & 1.01 & .22 & nd & 100.10 \\
\hline HR-86 & MK & $i$ & 1 & D-278085 & b & EWW & 50.80 & 17.20 & 11.20 & nd & 3.87 & 6.81 & 4.69 & 2.01 & .00 & 2.64 & .95 & .22 & nd & 100.39 \\
\hline HR-87 & MK & ly & 1 & D-278101 & b & EWW & 50.70 & 17.20 & 11.00 & nd & 3.71 & 6.81 & 4.64 & 2.03 & .17 & 2.53 & 1.01 & .22 & nd & 100.02 \\
\hline HR-88 & MK & lc & 1 & D-278088 & b & EWW & 51.20 & 17.10 & 10.80 & nd & 3.50 & 6.49 & 4.94 & 2.11 & .27 & 2.42 & 1.05 & .23 & nd & 100.11 \\
\hline HR-89 & MK & 1 & 1 & D-278089 & b & EWW & 50.80 & 17.20 & 11.20 & nd & 3.83 & 6.86 & 4.57 & 1.99 & .00 & 2.60 & .98 & .22 & nd & 100.25 \\
\hline HR-90 & MK & 1 & 1 & D-278102 & b & EWW & 51.10 & 17.00 & 10.70 & nd & 3.31 & 6.61 & 4.89 & 2.17 & .51 & 2.24 & 1.38 & .22 & nd & 100.13 \\
\hline HR-91 & MK & ly & 1 & D-278104 & b & EWW & 49.60 & 17.10 & 12.20 & nd & 4.36 & 7.24 & 4.32 & 1.75 & .04 & 2.91 & .83 & .21 & nd & 100.56 \\
\hline HR-92 & MK & $i$ & 1 & D-278090 & b & EWW & 50.40 & 16.90 & 11.50 & nd & 3.99 & 6.94 & 4.87 & 1.96 & .00 & 2.69 & .96 & .22 & nd & 100.43 \\
\hline HR-93 & MK & 1 & 1 & D-278091 & b & EWW & 51.00 & 17.00 & 11.10 & nd & 3.73 & 6.69 & 4.85 & 2.03 & .00 & 2.51 & .97 & .22 & nd & 100.10 \\
\hline HR-94 & MK & I & 1 & D-278086 & b & EWW & 50.30 & 16.90 & 11.80 & nd & 4.00 & 6.90 & 4.70 & 1.89 & .00 & 2.68 & .91 & .22 & nd & 100.30 \\
\hline HR-95 & MK & lc & 1 & D-278103 & b & EWW & 50.60 & 17.10 & 11.50 & nd & 3.80 & 6.63 & 4.71 & 1.93 & .00 & 2.54 & .97 & .23 & nd & 100.01 \\
\hline HR-96 & MK & lc & 1 & D-278087 & b & EWW & 50.90 & 17.00 & 11.20 & nd & 3.78 & 6.72 & 4.87 & 2.02 & .00 & 2.56 & .97 & .22 & nd & 100.24 \\
\hline HR-97 & MK & Iy & 1 & D-278092 & b & EWW & 51.00 & 17.10 & 11.30 & nd & 3.90 & 6.80 & 4.68 & 1.96 & .00 & 2.61 & .92 & .22 & nd $^{\circ}$ & 100.49 \\
\hline $\mathrm{HS}-1$ & MK & $i$ & 2 & D-277509 & b & WSW & 48.60 & 16.90 & 12.20 & nd & 4.44 & 7.22 & 4.30 & 1.74 & .26 & 3.02 & .89 & .22 & nd & 99.79 \\
\hline HS-2 & MK & i & 2 & D-277508 & b & WSW & 48.40 & 16.90 & 12.50 & nd & 4.56 & 7.40 & 4.28 & 1.70 & .06 & 3.16 & .85 & .21 & nd & 100.02 \\
\hline HS-3 & MK & 1 & 2 & D-277507 & b & WSW & 48.20 & 16.90 & 12.60 & nd & 4.80 & 7.51 & 4.33 & 1.66 & .00 & 3.25 & .82 & .21 & nd & 100.28 \\
\hline HS-4 & MK & 1 & 2 & D-277506 & b & WSW & 49.70 & 17.00 & 11.70 & nd & 4.02 & 6.95 & 4.60 & 1.86 & .05 & 2.79 & .95 & .22 & nd & 99.84 \\
\hline HS-5 & MK & ly & 2 & D-277505 & b & WSW & 49.40 & 16.90 & 12.00 & nd & 4.29 & 7.09 & 4.62 & 1.82 & .00 & 2.87 & .85 & .22 & nd & 100.06 \\
\hline HT-1 & MK & $i$ & 2 & D-277499 & b & WSW & 50.10 & 17.10 & 11.50 & nd & 3.71 & 6.59 & 4.55 & 1.99 & .12 & 2.59 & 1.03 & .23 & nd & 99.51 \\
\hline
\end{tabular}


Table 1.-Chemical analyses-Continued

\begin{tabular}{|c|c|c|c|c|c|c|c|c|c|c|c|c|c|c|c|c|c|c|c|c|}
\hline $\begin{array}{c}\text { Sample } \\
\text { locality } \\
\text { No. }\end{array}$ & $\begin{array}{l}\text { Vol- } \\
\text { cano }\end{array}$ & Unit & $\begin{array}{l}\text { Map } \\
\text { sheet } \\
\text { No. }\end{array}$ & $\begin{array}{l}\text { Laboratory } \\
\text { No. }\end{array}$ & Remark & Collector & $\mathrm{SiO}_{2}$ & $\mathrm{Al}_{2} \mathrm{O}_{3}$ & $\mathrm{Fe}_{2} \mathrm{O}_{3}$ & $\mathrm{FeO}$ & $\mathrm{MgO}$ & $\mathrm{CaO}$ & $\mathrm{Na}_{2} \mathrm{O}$ & $\mathrm{K}_{2} \mathrm{O}$ & $\mathrm{H}_{2} \mathrm{O}$ & $\mathrm{TO}_{2}$ & $\mathrm{P}_{2} \mathrm{O}_{5}$ & $\mathrm{MnO}$ & $\mathrm{CO}_{2}$ & Total \\
\hline HT-2 & $\mathrm{MK}$ & $\mathrm{hm}$ & 2 & D-277498 & $\mathrm{b}$ & WSW & 47.20 & 13.80 & 15.00 & $\overline{\text { nd }}$ & 5.62 & 10.60 & 2.78 & .84 & .00 & 3.83 & .49 & .20 & nd & 100.36 \\
\hline HU-1 & MK & $\mathrm{hm}$ & 2 & D-277500 & $b$ & WSW & 45.90 & 14.70 & 14.50 & nd & 6.39 & 10.50 & 2.54 & .62 & .46 & 3.83 & .35 & .19 & nd & 99.98 \\
\hline HU-2 & MK & $\mathrm{hm}$ & 2 & D-277497 & b & WSW & 46.80 & 13.60 & 15.50 & nd & 5.69 & 10.30 & 2.69 & .71 & .00 & 4.12 & .44 & .20 & nd & 100.05 \\
\hline HU-3 & MK & $\mathrm{hm}$ & 2 & D-277501 & b & WsW & 47.20 & 14.80 & 13.50 & nd & 6.57 & 11.30 & 2.29 & .35 & .34 & 3.13 & .34 & .19 & nd & 100.01 \\
\hline HU-4 & MK & $\mathrm{hm}$ & 2 & D-277493 & b & WSW & 46.90 & 14.10 & 14.80 & nd & 6.61 & 11.30 & 2.26 & .31 & .01 & 3.32 & .34 & .20 & nd & 100.15 \\
\hline HU-5 & MK & 1 & 2 & D-277495 & b & WSW & 49.40 & 17.10 & 11.80 & nd & 3.95 & 6.90 & 4.51 & 1.85 & .04 & 2.78 & .96 & .22 & nd & 99.51 \\
\hline HU-6 & MK & $\mathrm{hmc}$ & 2 & D-277496 & b & WSW & 40.20 & 13.20 & 18.00 & nd & 8.19 & 8.94 & 3.81 & 1.28 & .00 & 6.04 & .80 & .20 & nd & 100.66 \\
\hline $\mathrm{HU}-7$ & MK & $\mathrm{hm}$ & 2 & D-277491 & b & WSW & 46.00 & 10.10 & 12.60 & nd & 16.10 & 10.80 & 1.67 & .47 & .00 & 2.08 & .26 & .17 & nd & 100.25 \\
\hline HU-8 & MK & $\mathrm{hm}$ & 2 & D-277492 & b & WSW & 46.00 & 13.80 & 15.20 & nd & 5.08 & 9.26 & 3.01 & 1.20 & .50 & 4.85 & .65 & .20 & nd & 99.75 \\
\hline $\mathbb{N}-1$ & ML & k5-1859 & 1 & ML-59 & a & JMR & 50.37 & 12.64 & 12.44 & nd & 9.53 & 9.92 & 1.91 & .37 & nd & 2.00 & .22 & .17 & nd & 99.57 \\
\hline $\mathbb{N}-2$ & ML & k5-1859 & 1 & ML-58 & a & JMR & 50.63 & 12.67 & 12.45 & nd & 9.77 & 9.95 & 2.22 & .37 & nd & 1.98 & .22 & .18 & nd & 100.44 \\
\hline $10-1$ & MK & 1 & 1 & PH-1 & d & WSW & 49.48 & 16.91 & 11.85 & nd & 4.17 & 6.98 & 4.62 & 1.81 & .08 & 2.85 & .78 & .21 & nd & 99.74 \\
\hline $10-2$ & MK & 1 & 1 & D-260903 & b & WSW & 49.90 & 17.00 & 12.00 & nd & 4.26 & 7.14 & 4.74 & 1.82 & .00 & 2.92 & .84 & .22 & .00 & 100.84 \\
\hline $10-3$ & MK & $\mathrm{hm}$ & 1 & $115-3$ & d & WSW & 47.79 & 14.07 & 14.60 & nd & 5.17 & 10.24 & 2.83 & 1.00 & .31 & 3.54 & .49 & .20 & nd & 100.24 \\
\hline $10-4$ & MK & $\mathrm{hm}$ & 1 & $115-20$ & d & WSW & 47.01 & 13.73 & 13.67 & nd & 7.26 & 11.16 & 2.43 & .82 & .17 & 3.30 & .42 & .18 & nd & 100.15 \\
\hline 1O-5 & MK & $\mathrm{hm}$ & 1 & $115-4$ & d & WSW & 47.23 & 12.64 & 12.20 & nd & 11.23 & 11.63 & 1.68 & .50 & .13 & 2.13 & .27 & .16 & nd & 99.80 \\
\hline $10-6$ & MK & $\mathrm{hm}$ & 1 & $115-6$ & d & WSW & 45.73 & 14.61 & 15.83 & nd & 5.05 & 8.28 & 3.32 & 1.41 & .10 & 4.57 & .75 & .22 & nd & 99.87 \\
\hline 10-7 & MK & $\mathrm{hm}$ & 1 & D-260901 & b & WSW & 48.80 & 14.90 & 13.30 & nd & 5.81 & 10.50 & 2.96 & .93 & .00 & 3.44 & .47 & .18 & .00 & 101.29 \\
\hline 10-8 & MK & $\mathrm{hm}$ & 1 & $115-23$ & d & WSW & 47.60 & 14.21 & 13.30 & nd & 6.05 & 11.15 & 2.51 & .81 & .07 & 3.28 & .42 & .19 & nd & 99.59 \\
\hline 10-9 & MK & $\mathrm{hm}$ & 1 & $115-12$ & d & WSW & 45.17 & 10.20 & 12.44 & nd & 15.06 & 10.98 & 1.66 & .55 & .16 & 1.91 & .27 & .18 & nd & 98.58 \\
\hline $10-10$ & MK & $\mathrm{hm}$ & 1 & $115-14$ & d & WSW & 45.24 & 14.32 & 16.56 & nd & 5.09 & 8.13 & 3.34 & 1.43 & .15 & 4.71 & .74 & .23 & nd & 99.94 \\
\hline $10-11$ & MK & $\mathrm{hm}$ & 1 & $115-37$ & d & WSW & 47.60 & 13.52 & 14.44 & nd & 6.31 & 10.55 & 2.48 & .89 & .38 & 3.43 & .44 & .20 & nd & 100.24 \\
\hline $10-12$ & MK & $\mathrm{hm}$ & 1 & D-260902 & b & WSW & 44.80 & 14.20 & 17.30 & nd & 5.16 & 8.29 & 3.31 & 1.38 & .31 & 4.93 & .74 & .23 & .07 & 100.72 \\
\hline $10-13$ & MK & $\mathrm{hm}$ & 1 & $115-25$ & d & WSW & 47.84 & 13.77 & 14.81 & nd & 5.57 & 10.37 & 2.52 & .96 & .00 & 3.62 & .47 & .20 & nd & 100.13 \\
\hline $10-14$ & MK & $\mathrm{hm}$ & 1 & $115-36$ & d & WSW & 47.96 & 14.27 & 12.56 & nd & 7.20 & 11.76 & 2.13 & .67 & .11 & 2.77 & .32 & .18 & nd & 99.93 \\
\hline $10-15$ & MK & $\mathrm{hm}$ & 1 & 115-35 & $d$ & WSW & 48.26 & 14.32 & 12.34 & nd & 6.80 & 11.81 & 2.25 & .71 & .00 & 2.81 & .35 & .17 & nd & 99.82 \\
\hline $10-16$ & MK & $\mathrm{hm}$ & 1 & $115-11$ & d & WSW & 47.50 & 15.42 & 13.67 & nd & 5.25 & 11.02 & 2.74 & .83 & .17 & 3.38 & .40 & .19 & nd & 100.57 \\
\hline $\mathbb{P}-\mathbf{1}$ & MK & $\mathrm{hm}$ & 1 & D-260897 & b & WSW & 46.70 & 13.90 & 15.90 & nd & 5.60 & 10.70 & 2.83 & .74 & .00 & 3.96 & .41 & .21 & .00 & 100.95 \\
\hline IP-2 & MK & $\mathrm{hm}$ & 1 & NO-9 & d & WSW & 50.01 & 14.29 & 13.52 & nd & 4.26 & 8.78 & 3.29 & 1.35 & .29 & 3.11 & .75 & .21 & nd & 99.86 \\
\hline PP-3 & MK & $\mathrm{hm}$ & 1 & D-260887 & b & WSW & 46.60 & 14.20 & 14.10 & nd & 5.72 & 11.00 & 2.90 & .97 & .29 & 3.78 & .52 & .18 & .00 & 100.26 \\
\hline $\mathbb{P}-4$ & MK & lb & 1 & D-260888 & b & WSW & 54.70 & 17.60 & 9.13 & nd & 2.25 & 4.96 & 5.73 & 2.60 & .15 & 1.60 & .98 & .23 & .00 & 99.93 \\
\hline IP-5 & MK & $\mathrm{hm}$ & 1 & $73-4$ & d & WSW & 45.39 & 14.38 & 13.71 & nd & 8.04 & 10.93 & 2.54 & .85 & .20 & 3.26 & .42 & .19 & nd & 99.91 \\
\hline IP-6 & $\mathrm{MK}$ & $\mathrm{hm}$ & 1 & NO-11 & d & WSW & 47.34 & 15.74 & 13.09 & nd & 5.63 & 11.69 & 2.42 & .72 & .13 & 2.96 & .36 & .17 & nd & 100.25 \\
\hline IP-7 & MK & $\mathrm{hm}$ & 1 & NO-5 & d & WSW & 47.52 & 13.88 & 13.48 & nd & 6.91 & 11.35 & 2.57 & .82 & .07 & 3.32 & .40 & .19 & nd & 100.51 \\
\hline $\mathbb{P}$ P-8 & MK & $\mathrm{hm}$ & 1 & $83-17$ & d & WSW & 46.10 & 14.86 & 15.69 & nd & 5.15 & 8.31 & 3.13 & 1.42 & .34 & 4.56 & .75 & .22 & nd & 100.53 \\
\hline IP-9 & MK & $\mathrm{hm}$ & 1 & NO-13 & d & WSW & 45.54 & 14.44 & 13.81 & nd & 8.16 & 10.90 & 2.59 & .90 & .13 & 3.24 & .42 & .19 & nd & 100.32 \\
\hline $\mathbb{P}-10$ & MK & 1 & 1 & D-260882 & b & WSW & 49.90 & 17.10 & 12.10 & nd & 3.99 & 6.99 & 4.68 & 1.91 & .00 & 2.79 & 1.05 & .21 & .00 & 100.72 \\
\hline IP-11 & MK & $\mathrm{hm}$ & 1 & D-260889 & b & WSW & 47.30 & 13.60 & 14.50 & nd & 6.54 & 10.80 & 2.73 & .92 & .00 & 3.48 & .44 & .19 & .00 & 100.50 \\
\hline P-12 & MK & $\mathrm{hm}$ & 1 & NO-14 & d & WSW & 46.97 & 14.44 & 13.86 & nd & 5.74 & 10.73 & 2.93 & .98 & .19 & 3.71 & .49 & .19 & nd & 100.23 \\
\hline P -13 & MK & $\mathrm{hm}$ & 1 & D-260884 & b & WSW & 47.20 & 14.10 & 14.00 & nd & 6.14 & 10.90 & 3.00 & .99 & .00 & 3.75 & .51 & .18 & .00 & 100.77 \\
\hline
\end{tabular}


Table 1.-Chemical analyses-Continued

\begin{tabular}{|c|c|c|c|c|c|c|c|c|c|c|c|c|c|c|c|c|c|c|c|c|}
\hline $\begin{array}{c}\text { Sample } \\
\text { locality } \\
\text { No. }\end{array}$ & $\begin{array}{l}\text { Vol- } \\
\text { cano }\end{array}$ & Unit & $\begin{array}{c}\text { Map } \\
\text { sheet } \\
\text { No. }\end{array}$ & $\begin{array}{c}\text { Laboratory } \\
\text { No. }\end{array}$ & Remark & Collector & $\mathrm{SiO}_{2}$ & $\mathrm{Al}_{2} \mathrm{O}_{3}$ & $\mathrm{Fe}_{2} \mathrm{O}_{3}$ & $\mathrm{FeO}$ & $\mathrm{MgO}$ & $\mathrm{CaO}$ & $\mathrm{Na}_{2} \mathrm{O}$ & $\mathrm{K}_{2} \mathrm{O}$ & $\mathrm{H}_{2} \mathrm{O}$ & $\mathrm{TiO}_{2}$ & $\mathrm{P}_{2} \mathrm{O}_{5}$ & $\mathrm{MnO}$ & $\mathrm{CO}_{2}$ & Total \\
\hline IP-14 & MK & $\mathrm{hm}$ & 1 & $\mathrm{D}-260885$ & $\mathrm{~b}$ & WSW & 47.30 & 13.20 & 13.00 & nd & 9.73 & 11.80 & 2.23 & .66 & .00 & 2.76 & .34 & .17 & .00 & 101.19 \\
\hline IP-15 & MK & $\mathrm{hm}$ & 1 & $71-9$ & d & WSW & 47.47 & 13.98 & 13.91 & nd & 5.81 & 10.72 & 2.47 & .87 & .25 & 3.46 & .46 & .19 & nd & 99.59 \\
\hline IP-16 & $\mathrm{MK}$ & $\mathrm{hm}$ & 1 & $97-2$ & d & WSW & 47.48 & 14.14 & 12.82 & nd & 7.46 & 11.56 & 2.23 & .75 & .31 & 2.98 & .38 & .18 & nd & 100.29 \\
\hline IP-17 & $\mathrm{MK}$ & $\mathrm{hm}$ & 1 & D-260892 & $\mathrm{b}$ & WSW & 47.40 & 14.00 & 15.00 & nd & 5.61 & 10.80 & 2.79 & .93 & .00 & 3.56 & .45 & .20 & .01 & 100.75 \\
\hline IP-18 & $\mathrm{MK}$ & $\mathrm{hm}$ & 1 & D-260893 & $\mathrm{b}$ & WSW & 48.00 & 14.20 & 13.10 & nd & 6.60 & 11.60 & 2.53 & .80 & .19 & 3.17 & .40 & .18 & .00 & 100.77 \\
\hline IP-19 & MK & Ib & 1 & NO-10 & $d$ & WSW & 54.17 & 17.11 & 9.58 & nd & 2.63 & 5.00 & 5.48 & 2.55 & .14 & 1.60 & 1.07 & .22 & nd & 99.55 \\
\hline IP-20 & MK & $\mathrm{hm}$ & 1 & $83-27$ & d & WSW & 47.11 & 13.76 & 13.33 & nd & 7.79 & 10.92 & 2.75 & .83 & .13 & 3.27 & .44 & .18 & nd & 100.51 \\
\hline IP-21 & $\mathrm{MK}$ & $\mathrm{hm}$ & 1 & D-260898 & b & WSW & 47.40 & 13.90 & 12.60 & nd & 7.98 & 11.90 & 2.24 & .57 & .00 & 2.84 & .34 & .17 & .00 & 99.94 \\
\hline IP-22 & MK & 1 & 1 & D-271468 & b & WSW & 50.30 & 17.00 & 11.70 & nd & 4.00 & 6.99 & 4.80 & 1.96 & .00 & 2.76 & .96 & .22 & .00 & 100.69 \\
\hline $\mathbb{P}-23$ & MK & $\mathrm{hm}$ & 1 & D-260895 & b & WSW & 47.70 & 12.90 & 12.80 & nd & 9.84 & 11.70 & 2.14 & .69 & .00 & 2.60 & .31 & .17 & .00 & 100.85 \\
\hline IP-24 & MK & $\mathrm{hm}$ & 1 & $85-2$ & d & WSW & 47.51 & 13.75 & 13.17 & nd & 7.70 & 10.95 & 2.38 & .81 & .00 & 3.27 & .45 & .18 & nd & 100.17 \\
\hline IP-25 & MK & $\mathrm{hm}$ & 1 & D-260185 & $b$ & WSW & 46.80 & 14.10 & 13.70 & nd & 6.69 & 11.50 & 2.49 & .85 & .41 & 3.38 & .43 & .18 & .00 & 100.53 \\
\hline IP-26 & MK & $\mathrm{hm}$ & 1 & D-260186 & b & WSW & 47.50 & 13.40 & 13.00 & nd & 8.30 & 11.30 & 2.30 & .77 & .35 & 2.90 & .35 & .17 & .00 & 100.34 \\
\hline IQ-1 & MK & lb & 1 & D-276005 & b & MAK & 54.20 & 17.70 & 2.62 & 5.85 & 2.26 & 4.93 & 5.67 & 2.59 & .00 & 1.58 & .98 & .23 & .00 & 98.61 \\
\hline $\mathrm{IQ}-2$ & MK & 1 & 1 & D-276004 & b & MAK & 49.40 & 17.00 & 6.42 & 4.66 & 4.00 & 7.04 & 4.66 & 1.92 & .04 & 2.75 & .95 & .22 & .00 & 99.06 \\
\hline IQ-3 & MK & $\mathrm{hm}$ & 1 & D-276023 & $b$ & MAK & 47.10 & 14.50 & 4.03 & 8.70 & 6.74 & 12.00 & 2.44 & .52 & .00 & 3.27 & .37 & .18 & .00 & 99.85 \\
\hline IQ-4 & MK & 1 & 1 & D-276000 & $b$ & MAK & 50.60 & 17.10 & 4.31 & 6.10 & 3.74 & 6.82 & 4.86 & 2.04 & .08 & 2.60 & 1.03 & .21 & .00 & 99.49 \\
\hline $\mathrm{IQ}-5$ & MK & i & 1 & D-276003 & $b$ & MAK & 49.00 & 17.00 & 4.91 & 6.29 & 4.27 & 7.20 & 4.43 & 1.81 & .15 & 2.97 & .88 & .21 & .00 & 99.12 \\
\hline IQ-6 & MK & $\mathrm{hm}$ & 1 & D-276002 & b & MAK & 48.00 & 14.20 & 4.73 & 8.70 & 4.51 & 9.07 & 3.67 & 1.47 & .00 & 4.18 & .76 & .21 & .00 & 99.50 \\
\hline IQ-7 & MK & $\mathrm{hm}$ & 1 & D-275999 & $\mathrm{b}$ & MAK & 46.70 & 14.20 & 3.08 & 9.47 & 6.85 & 10.90 & 2.53 & .91 & .20 & 3.58 & .45 & .18 & .00 & 99.05 \\
\hline IQ-8 & MK & 1 & 1 & D-275998 & $\mathrm{b}$ & MAK & 49.80 & 17.00 & 7.76 & 3.46 & 4.00 & 7.10 & 4.75 & 1.92 & .26 & 2.74 & .95 & .21 & .02 & 99.97 \\
\hline 1Q-9 & $\mathrm{MK}$ & i & 1 & D-276001 & b & MAK & 52.90 & 17.60 & 3.20 & 6.39 & 2.61 & 5.10 & 5.23 & 2.49 & .25 & 1.76 & 1.16 & .24 & .00 & 98.93 \\
\hline IQ-10 & MK & $\mathrm{hmc}$ & 1 & D-260890 & b & WSW & 47.50 & 12.60 & 13.00 & nd & 9.95 & 11.90 & 2.12 & .59 & .26 & 2.66 & .32 & .17 & .00 & 101.07 \\
\hline IQ-11 & MK & 1 & 1 & D-275992 & $b$ & MAK & 49.20 & 17.00 & 4.31 & 6.92 & 4.34 & 7.23 & 4.57 & 1.82 & .24 & 2.97 & .92 & .21 & .00 & 99.73 \\
\hline IQ-12 & MK & $\mathrm{lbc}$ & 1 & D-275991 & $\mathrm{b}$ & MAK & 54.50 & 17.40 & 5.12 & 3.86 & 2.20 & 4.60 & 5.58 & 2.72 & .25 & 1.46 & 1.03 & .23 & .00 & 98.95 \\
\hline IQ-13 & MK & 1 & 1 & D-275996 & $\mathrm{b}$ & MAK & 49.90 & 17.00 & 5.91 & 5.12 & 3.97 & 6.98 & 4.78 & 1.94 & .00 & 2.75 & .98 & .22 & .00 & 99.55 \\
\hline IQ-14 & MK & $\mathrm{hm}$ & 1 & D-275997 & b & MAK & 47.40 & 14.00 & 4.94 & 7.97 & 6.34 & 11.50 & 2.55 & .84 & .00 & 3.40 & .41 & .18 & .00 & 99.53 \\
\hline IQ-15 & MK & $\mathrm{hmc}$ & 1 & D-275994 & b & MAK & 45.80 & 12.20 & 4.23 & 7.44 & 12.40 & 11.80 & 1.81 & .29 & .40 & 2.36 & .24 & .17 & .00 & 99.14 \\
\hline IQ-16 & $\mathrm{MK}$ & $\mathrm{hmc}$ & 1 & D-260177 & b & WSW & 47.60 & 15.20 & 13.20 & nd & 6.09 & 11.30 & 2.57 & .79 & .02 & 3.08 & .40 & .18 & .00 & 100.43 \\
\hline IQ-17 & $\mathrm{MK}$ & $\mathrm{hmc}$ & 1 & D-260176 & b & WSW & 46.80 & 13.40 & 12.60 & nd & 9.79 & 12.00 & 1.96 & .39 & .49 & 2.63 & .32 & .17 & .00 & 100.55 \\
\hline IQ-18 & MK & $\mathrm{hm}$ & 1 & D-260188 & $b$ & WSW & 47.30 & 14.30 & 13.40 & nd & 6.33 & 11.50 & 2.46 & .84 & .00 & 3.21 & .40 & .18 & .00 & 99.92 \\
\hline $\mathrm{IQ}-20$ & $\mathrm{MK}$ & 1 & 1 & D-275990 & b & MAK & 52.30 & 18.40 & 4.30 & 5.13 & 2.21 & 4.75 & 5.11 & 2.24 & 1.19 & 1.57 & 1.10 & .25 & .00 & 98.55 \\
\hline $\mathrm{IQ}-21$ & MK & $\mathrm{hm}$ & 1 & D-275995 & b & MAK & 45.10 & 11.30 & 3.77 & 8.11 & 13.70 & 12.00 & 1.39 & .22 & .66 & 2.29 & .24 & .17 & .00 & 98.95 \\
\hline IR-1 & MK & 1 & 1 & D-278153 & b & EWW & 50.70 & 17.20 & 11.10 & nd & 3.77 & 6.75 & 4.58 & 1.92 & .56 & 2.57 & 1.04 & .22 & nd & 100.41 \\
\hline IR-2 & MK & i & 1 & D-276013 & $b$ & MAK & 49.90 & 17.10 & 2.98 & 7.67 & 4.11 & 7.09 & 4.45 & 1.82 & .00 & 2.83 & .87 & .21 & .01 & 99.04 \\
\hline IR-3 & MK & $\mathrm{hm}$ & 1 & D-278150 & b & EWW & 47.40 & 13.50 & 15.50 & nd & 5.33 & 10.10 & 2.88 & 1.01 & .13 & 4.21 & .61 & .21 & nd & 100.88 \\
\hline IR-4 & $\mathrm{MK}$ & $\mathrm{hm}$ & 1 & D-278149 & b & EWW & 47.30 & 14.30 & 14.70 & nd & 6.00 & 11.10 & 2.83 & .75 & .00 & 3.58 & .45 & .19 & nd & 101.20 \\
\hline IR-5 & MK & $\mathrm{hm}$ & 1 & D-278151 & b & EWW & 48.60 & 13.50 & 15.70 & nd & 5.04 & 9.67 & 3.19 & 1.02 & .00 & 4.30 & .52 & .21 & nd & 101.75 \\
\hline IR-6 & MK & $\mathrm{hm}$ & 1 & D-278152 & b & EWW & 47.70 & 13.50 & 15.50 & nd & 5.24 & 9.93 & 2.97 & 1.01 & .00 & 4.22 & .54 & .21 & nd & 100.82 \\
\hline IR-7 & MK & ly & 1 & D-276012 & b & MAK & 50.70 & 17.10 & 3.75 & 6.88 & 3.92 & 6.73 & 4.77 & 1.95 & .00 & 2.62 & .90 & .22 & .00 & 99.54 \\
\hline
\end{tabular}


Table 1.-Chemical analyses-Continued

\begin{tabular}{|c|c|c|c|c|c|c|c|c|c|c|c|c|c|c|c|c|c|c|c|c|}
\hline $\begin{array}{l}\text { Sample } \\
\text { locality } \\
\text { No. }\end{array}$ & $\begin{array}{l}\text { Vol- } \\
\text { cano }\end{array}$ & Unit & $\begin{array}{l}\text { Map } \\
\text { sheet } \\
\text { No. }\end{array}$ & $\begin{array}{c}\text { Laboratory } \\
\text { No. }\end{array}$ & Remark & Collector & $\mathrm{SiO}_{2}$ & $\mathrm{Al}_{2} \mathrm{O}_{3}$ & $\mathrm{Fe}_{2} \mathrm{O}_{3}$ & $\mathrm{FeO}$ & $\mathrm{MgO}$ & $\mathrm{CaO}$ & $\mathrm{Na}_{2} \mathrm{O}$ & $\mathrm{K}_{2} \mathrm{O}$ & $\mathrm{H}_{2} \mathrm{O}$ & $\mathrm{TiO}_{2}$ & $\mathrm{P}_{2} \mathrm{O}_{5}$ & $\mathrm{MnO}$ & $\mathrm{CO}_{2}$ & Total \\
\hline IR-8 & MK & 1 & 1 & D-276014 & $\bar{b}$ & MAK & 49.50 & 16.90 & 3.54 & 7.43 & 4.22 & 7.34 & 4.55 & 1.83 & .00 & 2.89 & .87 & .22 & .00 & 99.29 \\
\hline IR-9 & $\mathrm{MK}$ & $i$ & 1 & D-276018 & b & MAK & 50.00 & 17.10 & 3.33 & 6.90 & 3.61 & 6.73 & 4.75 & 1.86 & .75 & 2.49 & 1.01 & .22 & .00 & 98.75 \\
\hline IR-10 & $\mathrm{MK}$ & $i$ & 1 & D-276011 & b & MAK & 50.40 & 17.10 & 2.71 & 7.46 & 3.46 & 6.76 & 4.65 & 2.07 & .00 & 2.37 & 1.25 & .22 & .00 & 98.45 \\
\hline IR-11 & MK & $i$ & 1 & D-276015 & b & MAK & 50.70 & 17.00 & 2.80 & 7.20 & 3.51 & 6.68 & 4.73 & 2.02 & .00 & 2.45 & 1.06 & .22 & .00 & 98.37 \\
\hline IR-12 & $\mathrm{MK}$ & $i$ & 1 & D-276016 & b & MAK & 51.20 & 17.20 & 3.39 & 6.58 & 3.50 & 6.71 & 4.97 & 2.12 & .00 & 2.48 & 1.08 & .22 & .00 & 99.45 \\
\hline IR-13 & $\mathrm{MK}$ & $\mathrm{Ibc}$ & 1 & D-276019 & b & MAK & 55.10 & 17.40 & 2.71 & 5.87 & 2.18 & 4.34 & 5.51 & 2.73 & .00 & 1.37 & .97 & .23 & .00 & 98.41 \\
\hline IR-14 & MK & 1 & 1 & D-276021 & b & MAK & 49.60 & 17.00 & 4.25 & 6.79 & 4.23 & 7.13 & 4.76 & 1.89 & .00 & 2.84 & .93 & .21 & .00 & 99.63 \\
\hline IR-15 & $\mathrm{MK}$ & 1 & 1 & D-276020 & $\mathrm{b}$ & MAK & 49.90 & 17.00 & 2.20 & 8.37 & 4.12 & 7.02 & 4.65 & 1.91 & .00 & 2.85 & .88 & .21 & .00 & 99.11 \\
\hline IR-16 & $\mathrm{MK}$ & $\mathrm{hm}$ & 1 & D-276022 & b & MAK & 46.80 & 13.80 & 4.84 & 8.96 & 6.11 & 9.74 & 3.20 & 1.21 & .00 & 4.07 & .60 & .20 & .00 & 99.53 \\
\hline IR-17 & MK & Iy & 1 & D-276017 & b & MAK & 50.70 & 17.00 & 3.69 & 7.03 & 3.86 & 6.71 & 4.77 & 1.98 & .00 & 2.60 & .94 & .22 & .00 & 99.50 \\
\hline $\mathbb{R}-18$ & MK & 1 & 1 & D-276006 & b & MAK & 50.30 & 17.00 & 4.07 & 6.51 & 3.85 & 6.88 & 4.61 & 1.94 & .00 & 2.65 & .95 & .22 & .00 & 98.98 \\
\hline IR-19 & $\mathrm{MK}$ & 1 & 1 & D-276008 & b & MAK & 48.70 & 17.00 & 6.78 & 4.52 & 3.93 & 7.07 & 4.37 & 1.84 & .49 & 2.81 & .95 & .21 & .00 & 98.67 \\
\hline IR-20 & MK & i & 1 & D-276009 & b & MAK & 49.30 & 17.10 & 5.56 & 5.62 & 3.87 & 7.01 & 4.54 & 1.87 & .24 & 2.77 & .99 & .22 & .00 & 99.09 \\
\hline IR-21 & MK & 1 & 1 & D-276010 & b & MAK & 49.50 & 17.10 & 2.64 & 8.33 & 3.94 & 7.04 & 4.49 & 1.89 & .15 & 2.82 & .98 & .22 & .00 & 99.10 \\
\hline IS- 1 & $\mathrm{MK}$ & $i$ & 2 & D-277503 & b & WSW & 51.30 & 17.00 & 11.10 & nd & 3.55 & 6.43 & 4.85 & 2.07 & .00 & 2.41 & 1.02 & .23 & nd & 99.96 \\
\hline IS-2 & $\mathrm{MK}$ & $i$ & 2 & D-277504 & b & WSW & 51.40 & 17.20 & 11.00 & nd & 3.60 & 6.54 & 4.78 & 2.04 & .00 & 2.42 & 1.02 & .23 & nd & 100.23 \\
\hline IS-3 & MK & $\mathrm{hm}$ & 2 & D-277502 & b & WSW & 45.40 & 13.50 & 14.20 & nd & 8.69 & 11.60 & 2.04 & .25 & 1.00 & 3.18 & .39 & .20 & nd & 100.45 \\
\hline IS -4 & $\mathrm{MK}$ & ly & 2 & D-271467 & b & WSW & 50.40 & 16.90 & 11.80 & nd & 3.81 & 6.79 & 4.68 & 1.90 & .12 & 2.67 & .92 & .23 & .00 & 100.22 \\
\hline$\pi-1$ & MK & $\mathrm{hm}$ & 2 & D-271471 & b & WSW & 46.20 & 14.90 & 14.50 & nd & 6.73 & 10.80 & 2.57 & .61 & .20 & 3.86 & .34 & .19 & .00 & 100.90 \\
\hline IT-2 & MK & 1 & 2 & D-271470 & b & WSW & 49.30 & 17.00 & 12.10 & nd & 4.09 & 7.02 & 4.49 & 1.80 & .24 & 2.86 & .93 & .22 & .00 & 100.05 \\
\hline $\mathrm{T}-3$ & MK & $\mathrm{hm}$ & 2 & D-271473 & b & WSW & 47.00 & 13.10 & 13.40 & nd & 9.70 & 11.10 & 2.18 & .68 & .45 & 2.93 & .36 & .18 & .00 & 101.08 \\
\hline$\Pi-4$ & MK & $\mathrm{hm}$ & 2 & D-271472 & b & WSW & 47.00 & 12.20 & 14.00 & nd & 11.30 & 9.73 & 2.37 & .62 & .01 & 2.98 & .39 & .18 & .00 & 100.78 \\
\hline IT-5 & MK & $\mathrm{hm}$ & 2 & D-271476 & b & WSW & 47.40 & 14.40 & 14.80 & nd & 6.17 & 10.90 & 2.63 & .67 & .00 & 3.51 & .43 & .20 & .00 & 101.11 \\
\hline$\pi-6$ & $\mathrm{MK}$ & $\mathrm{hm}$ & 2 & D-271474 & b & WSW & 46.50 & 14.30 & 15.50 & nd & 5.87 & 10.30 & 2.59 & .73 & .64 & 3.74 & .46 & .21 & .00 & 100.84 \\
\hline Tा-7 & $\mathrm{MK}$ & $\mathrm{hm}$ & 2 & D-271478 & b & WSW & 47.20 & 14.00 & 14.50 & nd & 5.20 & 10.20 & 3.16 & 1.11 & .00 & 4.84 & .63 & .20 & .00 & 101.04 \\
\hline IT-8 & $\mathrm{MK}$ & $\mathrm{hm}$ & 2 & D-271475 & b & WSW & 47.70 & 13.90 & 12.70 & nd & 8.43 & 12.50 & 2.24 & .57 & .00 & 2.65 & .32 & .17 & .00 & 101.18 \\
\hline IT-9 & MK & $\mathrm{hm}$ & 2 & D-271477 & b & WSW & 44.60 & 16.30 & 16.00 & nd & 5.61 & 8.39 & 3.05 & 1.17 & .20 & 4.22 & .62 & .21 & .00 & 100.37 \\
\hline $\mathrm{JN}-1$ & $\mathrm{KO}$ & hw & 1 & D-269449 & b & MOG & 51.90 & 16.90 & 10.70 & nd & 3.38 & 5.72 & 5.71 & 2.06 & .00 & 2.11 & 1.47 & .25 & .00 & 100.20 \\
\hline $\mathrm{JN}-2$ & $\mathrm{KO}$ & p & 1 & D-269454 & $b$ & MOG & 48.70 & 14.40 & 14.40 & nd & 5.02 & 9.48 & 3.22 & 1.04 & .18 & 3.66 & .55 & .19 & .00 & 100.84 \\
\hline $\mathrm{JN}-3$ & $\mathrm{KO}$ & pi & 1 & D-269450 & b & MOG & 47.40 & 16.70 & 13.40 & nd & 5.16 & 7.75 & 4.32 & 1.44 & .00 & 3.72 & .83 & .18 & .00 & 100.90 \\
\hline $\mathrm{JN}-4$ & $\mathrm{KO}$ & $\mathrm{pl}$ & 1 & D-269456 & b & MOG & 45.90 & 13.00 & 13.80 & nd & 11.50 & 10.70 & 2.30 & .59 & .34 & 2.61 & .35 & .18 & .50 & 101.77 \\
\hline $\mathrm{JN}-5$ & $\mathrm{KO}$ & pl & 1 & D-269489 & b & MOG & 46.00 & 12.70 & 14.40 & nd & 12.70 & 9.87 & 2.25 & .59 & .00 & 2.58 & .33 & .18 & .09 & 101.69 \\
\hline JN-6 & $\mathrm{KO}$ & $\mathrm{pl}$ & 1 & D-269455 & b & MOG & 48.30 & 14.00 & 11.90 & nd & 9.90 & 11.90 & 2.11 & .39 & .32 & 2.20 & .25 & .16 & .04 & 101.47 \\
\hline $\mathrm{JN}-7$ & $\mathrm{KO}$ & hw & 1 & D-269451 & b & MOG & 47.10 & 15.90 & 13.10 & nd & 4.43 & 7.78 & 4.91 & 1.61 & .00 & 3.05 & 2.21 & .23 & .00 & 100.32 \\
\hline $\mathrm{JN}-8$ & $\mathrm{KO}$ & pl & 1 & D-269470 & b & MOG & 47.90 & 14.10 & 15.40 & nd & 4.70 & 9.61 & 3.29 & 1.12 & .13 & 4.03 & .61 & .21 & .00 & 101.10 \\
\hline $\mathrm{JN}-9$ & $\mathrm{KO}$ & pl & 1 & D-269474 & b & MOG & 48.00 & 13.90 & 15.60 & nd & 4.88 & 9.92 & 3.17 & .97 & .00 & 3.94 & .56 & .21 & .00 & 101.15 \\
\hline $\mathrm{JN}-10$ & $\mathrm{KO}$ & $\mathrm{pl}$ & 1 & D-269460 & b & MOG & 48.50 & 14.60 & 14.40 & nd & 4.71 & 9.10 & 3.77 & 1.29 & .00 & 3.83 & .68 & .21 & .00 & 101.09 \\
\hline JO-1 & MK & $\mathrm{hm}$ & 1 & $114-9$ & d & WSW & 47.82 & 12.60 & 12.07 & nd & 10.66 & 11.72 & 1.71 & .54 & .23 & 2.40 & .30 & .16 & nd & 100.21 \\
\hline $\mathrm{JO}-2$ & $\mathrm{MK}$ & $\mathrm{hm}$ & 1 & D-260900 & b & WSW & 48.50 & 14.20 & 13.60 & nd & 6.56 & 11.50 & 2.56 & .71 & .00 & 3.09 & .37 & .18 & .00 & 101.27 \\
\hline JO-3 & $\mathrm{KO}$ & hw & 1 & D-260746 & b & MOG & 47.60 & 15.90 & 13.20 & nd & 4.59 & 7.56 & 4.85 & 1.59 & .00 & 3.02 & 2.28 & .23 & .00 & 100.82 \\
\hline
\end{tabular}


Table 1.-Chemical analyses-Continued

\begin{tabular}{|c|c|c|c|c|c|c|c|c|c|c|c|c|c|c|c|c|c|c|c|c|}
\hline $\begin{array}{c}\text { Sample } \\
\text { locality } \\
\text { No. }\end{array}$ & $\begin{array}{l}\text { Vol- } \\
\text { cano }\end{array}$ & Unit & $\begin{array}{c}\text { Map } \\
\text { sheet } \\
\text { No. }\end{array}$ & $\begin{array}{l}\text { Laboratory } \\
\text { No. }\end{array}$ & Remark & Collector & $\mathrm{SiO}_{2}$ & $\mathrm{Al}_{2} \mathrm{O}_{3}$ & $\mathrm{Fe}_{2} \mathrm{O}_{3}$ & $\mathrm{FeO}$ & $\mathrm{MgO}$ & $\mathrm{CaO}$ & $\mathrm{Na}_{2} \mathrm{O}$ & $\mathrm{K}_{2} \mathrm{O}$ & $\mathrm{H}_{2} \mathrm{O}$ & $\mathrm{TiO}_{2}$ & $\mathrm{P}_{2} \mathrm{O}_{5}$ & $\mathrm{MnO}$ & $\mathrm{CO}_{2}$ & Total \\
\hline JO-4 & $\mathrm{KO}$ & $\mathrm{pl}$ & 1 & D-260740 & $\bar{b}$ & $\overline{\text { MOG }}$ & 48.40 & 13.20 & 16.00 & nd & 5.33 & 10.00 & 2.58 & .66 & .00 & 3.78 & .49 & .21 & .00 & 100.65 \\
\hline JO-5 & MK & $\mathrm{hm}$ & 1 & KW-1 & $d$ & WSW & 47.06 & 13.54 & 13.25 & nd & 7.68 & 11.34 & 2.46 & .84 & .09 & 3.12 & .43 & .19 & nd & 100.00 \\
\hline JO-6 & $\mathrm{KO}$ & hwb & 1 & D-260745 & b & MOG & 54.50 & 17.40 & 9.45 & nd & 2.62 & 4.77 & 6.00 & 2.35 & .00 & 1.74 & 1.22 & .25 & .00 & 100.30 \\
\hline JO-7 & $\mathrm{MK}$ & $\mathrm{hm}$ & 1 & D-260744 & b & MOG & 47.90 & 14.00 & 14.40 & nd & 5.58 & 10.10 & 3.13 & 1.13 & .00 & 4.06 & .55 & .20 & .00 & 101.05 \\
\hline JO-8 & KO & $\mathrm{pl}$ & 1 & D-269447 & b & MOG & 48.40 & 14.50 & 13.60 & nd & 6.06 & 10.50 & 2.60 & .44 & .94 & 3.00 & .40 & .19 & .00 & 100.63 \\
\hline JO-9 & $\mathrm{KO}$ & plc & 1 & D-260731 & b & MOG & 50.60 & 14.00 & 13.40 & nd & 5.74 & 10.50 & 2.48 & .67 & .00 & 3.00 & .41 & .19 & .00 & 100.99 \\
\hline JO-10 & $\mathrm{KO}$ & $\mathrm{pl}$ & 1 & D-260732 & $b$ & MOG & 48.30 & 14.80 & 13.60 & nd & 7.10 & 11.00 & 2.11 & .14 & .69 & 2.74 & .29 & .19 & .00 & 100.96 \\
\hline JO-11 & $\mathrm{KO}$ & $\mathrm{pl}$ & 1 & D-260733 & b & MOG & 51.20 & 13.70 & 11.80 & nd & 8.07 & 10.90 . & 2.26 & .16 & .29 & 2.12 & .20 & .17 & .00 & 100.87 \\
\hline JO-12 & $\mathrm{KO}$ & $\mathrm{pl}$ & 1 & D-260734 & b & MOG & 50.70 & 13.80 & 12.20 & nd & 7.63 & 10.80 & 2.21 & .12 & .80 & 2.50 & .23 & .18 & .00 & 101.17 \\
\hline JO-13 & $\mathrm{KO}$ & $\mathrm{pl}$ & 1 & D-260735 & b & MOG & 48.70 & 13.80 & 12.80 & nd & 9.47 & 10.10 & 1.86 & .10 & 1.15 & 2.23 & .21 & .18 & .00 & 100.60 \\
\hline JO-14 & $\mathrm{KO}$ & $\mathrm{pl}$ & 1 & D-260736 & b & MOG & 51.80 & 14.00 & 12.10 & nd & 6.91 & 11.00 & 2.24 & .32 & .00 & 2.28 & .22 & .17 & .00 & 101.04 \\
\hline JO-15 & $\mathrm{KO}$ & $\mathrm{pl}$ & 1 & D-277479 & b & $\mathrm{SCP}$ & 47.70 & 15.60 & 13.20 & nd & 5.59 & 9.36 & 3.54 & 1.24 & .06 & 3.30 & .65 & .18 & nd & 100.42 \\
\hline JO-16 & $\mathrm{KO}$ & $\mathrm{pl}$ & 1 & D-260737 & b & MOG & 50.90 & 14.10 & 12.50 & nd & 7.28 & 11.30 & 2.19 & .12 & .24 & 2.19 & .20 & .18 & .00 & 101.20 \\
\hline JO-17 & $\mathrm{KO}$ & $\mathrm{pl}$ & 1 & D-260738 & b & MOG & 48.70 & 10.40 & 13.10 & nd & 15.90 & 8.66 & 1.61 & .25 & .04 & 1.88 & .17 & .17 & .00 & 100.88 \\
\hline JO-18 & KO & $\mathrm{pl}$ & 1 & D-260729 & b & MOG & 50.40 & 13.80 & 14.00 & nd & 5.82 & 9.68 & 2.75 & .68 & .02 & 3.27 & .44 & .19 & .00 & 101.05 \\
\hline JO-19 & $\mathrm{KO}$ & $\mathrm{pl}$ & 1 & D-260730 & b & MOG & 46.60 & 15.70 & 14.50 & nd & 5.50 & 10.80 & 2.51 & .23 & .99 & 3.25 & .42 & .18 & .00 & 100.68 \\
\hline JO-20 & KO & pl & 1 & D-260749 & b & MOG & 47.80 & 15.10 & 13.10 & nd & 6.73 & 11.40 & 2.63 & .65 & .02 & 3.06 & .39 & .17 & .00 & 101.05 \\
\hline JO-25 & $\mathrm{KO}$ & pl & 1 & D-260728 & b & MOG & 48.80 & 16.40 & 12.30 & nd & 4.49 & 9.69 & 3.45 & 1.24 & .37 & 3.16 & .63 & .18 & .13 & 100.84 \\
\hline JO-26 & $\mathrm{KO}$ & hwb & 1 & D-260719 & b & MOG & 57.10 & 18.20 & 7.60 & nd & 1.62 & 3.11 & 6.48 & 2.74 & .76 & 1.18 & .81 & .24 & .00 & 99.84 \\
\hline JO-27 & $\mathrm{KO}$ & $\mathrm{pl}$ & 1 & D-260750 & $b$ & MOG & 48.20 & 15.50 & 13.60 & nd & 6.07 & 11.20 & 2.23 & .14 & .78 & 2.71 & .31 & .19 & .00 & 100.93 \\
\hline $\mathrm{JO}-28$ & $\mathrm{KO}$ & hw & 1 & D-260720 & b & MOG & 52.80 & 17.20 & 10.40 & nd & 3.10 & 5.59 & 5.60 & 2.12 & .00 & 2.03 & 1.47 & .24 & .00 & 100.55 \\
\hline JO-29 & $\mathrm{KO}$ & $\mathrm{pl}$ & 1 & D-260741 & b & MOG & 48.80 & 14.90 & 13.30 & nd & 4.94 & 10.00 & 3.47 & 1.14 & .22 & 3.43 & .58 & .20 & .00 & 100.98 \\
\hline JO-30 & $\mathrm{KO}$ & hwb & 1 & D-260723 & b & MOG & 55.00 & 17.90 & 8.65 & nd & 2.18 & 4.00 & 5.66 & 2.82 & .95 & 1.73 & .99 & .21 & .00 & 100.09 \\
\hline JO-31 & $\mathrm{KO}$ & plc & 1 & D-260739 & b & MOG & 48.70 & 15.80 & 12.80 & nd & 4.97 & 10.40 & 3.32 & 1.07 & .00 & 3.28 & .57 & .18 & .00 & 101.09 \\
\hline $\mathrm{JO}-32$ & $\mathrm{KO}$ & hw & 1 & D-260721 & b & MOG & 49.30 & 16.60 & 12.00 & nd & 3.94 & 7.04 & 5.00 & 1.76 & .09 & 2.63 & 1.96 & .23 & .00 & 100.55 \\
\hline JO-33 & $\mathrm{KO}$ & $\mathrm{pl}$ & 1 & D-260742 & b & MOG & 48.30 & 14.60 & 13.00 & nd & 6.72 & 11.70 & 2.72 & .66 & .00 & 2.99 & .39 & .18 & .00 & 101.26 \\
\hline JO-34 & $\mathrm{KO}$ & hw & 1 & D-260722 & b & MOG & 47.80 & 17.80 & 12.60 & nd & 2.86 & 5.87 & 4.56 & 1.72 & 2.02 & 2.71 & 1.94 & .22 & .00 & 100.10 \\
\hline JO-35 & $\mathrm{KO}$ & $\mathrm{pl}$ & 1 & D-260743 & b & MOG & 48.70 & 16.20 & 12.60 & nd & 4.95 & 10.70 & 3.28 & 1.03 & .00 & 3.23 & .54 & .17 & .00 & 101.40 \\
\hline JO-36 & $\mathrm{KO}$ & hwc & 1 & D-260726 & b & MOG & 53.50 & 17.50 & 9.66 & nd & 2.69 & 4.95 & 5.76 & 2.24 & .83 & 1.87 & 1.30 & .25 & .00 & 100.55 \\
\hline JO-37 & KO & hwb & 1 & D-260748 & b & MOG & 57.40 & 19.70 & 6.82 & nd & .85 & 1.70 & 5.88 & 3.04 & 2.57 & .91 & .37 & .23 & .00 & 99.47 \\
\hline $\mathrm{JO}-38$ & $\mathrm{KO}$ & hwc & 1 & D-260725 & b & MOG & 47.10 & 18.00 & 13.10 & nd & 2.99 & 5.77 & 4.19 & 1.74 & 2.41 & 2.83 & 2.01 & .28 & .00 & 100.42 \\
\hline JO-39 & $\mathrm{KO}$ & hwc & 1 & D-260747 & b & MOG & 53.20 & 18.00 & 9.98 & nd & 2.25 & 4.85 & 5.47 & 2.20 & 1.04 & 1.89 & 1.40 & .26 & .00 & 100.54 \\
\hline $\mathrm{JO}-40$ & $\mathrm{KO}$ & hw & 1 & D-260724 & b & MOG & 51.90 & 17.90 & 9.80 & nd & 2.47 & 5.07 & 5.24 & 2.19 & 1.63 & 2.32 & 1.11 & .26 & .00 & 99.89 \\
\hline JO-41 & $\mathrm{KO}$ & hw & 1 & D-283163 & b & MOG & 48.40 & 16.50 & 12.40 & nd & 3.86 & 6.85 & 4.57 & 1.73 & 1.05 & 2.70 & 1.95 & .24 & nd & 100.25 \\
\hline JP-1 & $\mathrm{MK}$ & $\mathrm{hm}$ & 1 & $85-10$ & d & WSW & 47.10 & 10.12 & 12.72 & nd & 14.35 & 10.83 & 1.63 & .57 & .07 & 2.32 & .30 & .18 & nd & 100.19 \\
\hline JP-2 & MK & $\mathrm{hm}$ & 1 & D-260187 & b & WSW & 47.90 & 14.20 & 13.80 & nd & 6.20 & 11.20 & 2.56 & .70 & .00 & 3.17 & .38 & .19 & .00 & 100.30 \\
\hline JP-5 & $\mathrm{KO}$ & hw & 1 & D-261417 & b & SCP & 47.80 & 16.60 & 12.90 & nd & 3.50 & 6.78 & 4.55 & 1.82 & 1.09 & 2.85 & 2.13 & .23 & .00 & 100.25 \\
\hline JP-7 & MK & $\mathrm{hm}$ & 1 & D-277461 & b & SCP & 48.70 & 13.90 & 13.10 & nd & 6.99 & 11.50 & 2.33 & .64 & .05 & 2.84 & .35 & .17 & nd & 100.57 \\
\hline JP-8 & $\mathrm{KO}$ & hwc & 1 & D-261429 & b & SCP & 49.00 & 17.20 & 12.20 & nd & 3.61 & 6.29 & 4.49 & 1.77 & .91 & 2.66 & 1.87 & .24 & .00 & 100.24 \\
\hline JP-9 & $\mathrm{KO}$ & hw & 1 & D-261397 & b & SCP & 48.70 & 16.50 & 12.30 & nd & 4.07 & 7.09 & 4.93 & 1.74 & .00 & 2.71 & 2.05 & .24 & .00 & 100.33 \\
\hline
\end{tabular}


Table 1.-Chemical analyses-Continued

\begin{tabular}{|c|c|c|c|c|c|c|c|c|c|c|c|c|c|c|c|c|c|c|c|c|c|}
\hline $\begin{array}{c}\text { Sample } \\
\text { locality } \\
\text { No. }\end{array}$ & $\begin{array}{l}\text { Vol- } \\
\text { cano }\end{array}$ & Unit & & $\begin{array}{c}\text { Map } \\
\text { sheet } \\
\text { No. }\end{array}$ & $\begin{array}{c}\text { Laboratory } \\
\text { No. }\end{array}$ & Remark & Collector & $\mathrm{SiO}_{2}$ & $\mathrm{Al}_{2} \mathrm{O}_{3}$ & $\mathrm{Fe}_{2} \mathrm{O}_{3}$ & $\mathrm{FeO}$ & $\mathrm{MgO}$ & $\mathrm{CaO}$ & $\mathrm{Na}_{2} \mathrm{O}$ & $\mathrm{K}_{2} \mathrm{O}$ & $\mathrm{H}_{2} \mathrm{O}$ & $\mathrm{TiO}_{2}$ & $\mathrm{P}_{2} \mathrm{O}_{5}$ & $\mathrm{MnO}$ & $\mathrm{CO}_{2}$ & Total \\
\hline JP-10 & $\mathrm{KO}$ & hw & & 1 & D-261428 & $\mathrm{b}$ & SCP & 49.30 & 16.60 & 12.10 & nd & 3.45 & 6.14 & 4.80 & 1.79 & .40 & 2.57 & 1.97 & .24 & .00 & 99.36 \\
\hline JP-12 & $\mathrm{KO}$ & hw & & 1 & D-261424 & $\mathrm{b}$ & $\mathrm{SCP}$ & 49.10 & 17.30 & 11.60 & nd & 3.27 & 6.60 & 4.47 & 1.90 & 1.00 & 2.65 & 1.67 & .27 & .00 & 99.83 \\
\hline JP-13 & $\mathrm{KO}$ & $\mathrm{pl}$ & & 1 & D-269114 & $\mathrm{b}$ & SCP & 50.30 & 13.50 & 14.70 & nd & 4.27 & 7.93 & 2.90 & 1.17 & .96 & 3.69 & .67 & .21 & .00 & 100.30 \\
\hline JP-15 & $\mathrm{KO}$ & hw & & 1 & D-261410 & $b$ & SCP & 47.40 & 16.00 & 13.20 & nd & 4.57 & 7.53 & 4.78 & 1.58 & .00 & 3.07 & 2.25 & .23 & .00 & 100.61 \\
\hline JP-16 & $\mathrm{KO}$ & plc & & 1 & D-261427 & $b$ & SCP & 48.90 & 17.90 & 12.20 & nd & 3.63 & 6.29 & 4.31 & 1.65 & 1.30 & 3.12 & 1.12 & .21 & .00 & 100.63 \\
\hline JP-17 & $\mathrm{KO}$ & $\mathrm{pl}$ & & 1 & D-269155 & b & SCP & 48.10 & 15.80 & 13.20 & nd & 4.44 & 9.37 & 3.42 & 1.35 & .65 & 3.31 & .66 & .20 & .00 & 100.50 \\
\hline JP-18 & $\mathrm{KO}$ & $\mathrm{pl}$ & & 1 & D-269153 & b & $\mathrm{SCP}$ & 46.70 & 14.50 & 14.90 & nd & 5.09 & 9.30 & 2.80 & 1.09 & .95 & 4.30 & .69 & .20 & .00 & 100.52 \\
\hline JP-19 & $\mathrm{KO}$ & pl & & 1 & D-269115 & b & SCP & 51.10 & 13.70 & 14.60 & nd & 3.68 & 7.74 & 3.14 & 1.27 & .84 & 3.42 & .81 & .22 & .00 & 100.52 \\
\hline JP-20 & $\mathrm{KO}$ & pl & & 1 & D-269156 & $\mathrm{b}$ & SCP & 46.90 & 15.50 & 13.20 & nd & 6.33 & 10.90 & 2.66 & .79 & .70 & 3.17 & .45 & .17 & .02 & 100.79 \\
\hline JP-21 & $\mathrm{KO}$ & hwb & & 1 & D-261425 & $\mathrm{b}$ & SCP & 57.10 & 17.80 & 7.69 & nd & 1.54 & 3.46 & 6.02 & 3.07 & .76 & 1.46 & .89 & .17 & .00 & 99.96 \\
\hline JP-22 & $\mathrm{KO}$ & hw & & 1 & D-269164 & $\mathrm{b}$ & SCP & 48.30 & 16.50 & 12.70 & nd & 4.06 & 7.10 & 4.49 & 1.74 & .39 & 2.82 & 2.07 & .24 & .00 & 100.41 \\
\hline JP-23 & KO & hwb & & 1 & D-261423 & $\mathrm{b}$ & SCP & 53.80 & 18.00 & 9.24 & nd & 2.04 & 4.59 & 5.82 & 2.30 & .68 & 1.81 & 1.25 & .24 & .01 & 99.78 \\
\hline JP-24 & $\mathrm{KO}$ & pl & & 1 & D-261418 & b & SCP & 46.70 & 14.50 & 14.70 & nd & 5.04 & 8.81 & 2.66 & 1.05 & 2.16 & 4.18 & .66 & .19 & .00 & 100.65 \\
\hline JP-25 & $\mathrm{KO}$ & hwb & & 1 & D-261426 & $b$ & SCP & 58.50 & 17.50 & 6.65 & nd & 1.49 & 2.87 & 6.34 & 3.41 & 1.06 & 1.17 & .72 & .18 & .00 & 99.89 \\
\hline JP-26 & $\mathrm{KO}$ & hwt & & 1 & D-261403 & $\mathrm{b}$ & SCP & 59.10 & 17.80 & 5.72 & nd & 1.18 & 2.59 & 6.60 & 3.49 & 1.56 & .95 & .62 & .19 & .01 & 99.81 \\
\hline JP-28 & $\mathrm{KO}$ & hwb & & 1 & D-261402 & b & SCP & 53.80 & 17.20 & 9.15 & nd & 2.44 & 5.14 & 5.60 & 2.56 & .57 & 1.92 & 1.30 & .19 & .00 & 99.87 \\
\hline JP-29 & $\mathrm{KO}$ & hw & & 1 & D-261409 & $\mathrm{b}$ & $\mathrm{SCP}$ & 46.90 & 16.00 & 13.20 & nd & 4.19 & 7.51 & 4.61 & 1.58 & .60 & 3.06 & 2.35 & .23 & .00 & 100.23 \\
\hline JP-30 & $\mathrm{KO}$ & hw & & 1 & D-261400 & b & SCP & 52.00 & 17.10 & 10.70 & nd & 3.38 & 5.95 & 5.45 & 2.08 & .00 & 2.20 & 1.59 & .24 & .00 & 100.69 \\
\hline JP-31 & KO & $\mathrm{pl}$ & & 1 & D-269152 & b & $\mathrm{SCP}$ & 47.40 & 15.60 & 12.80 & nd & 6.80 & 10.60 & 2.89 & .94 & .00 & 2.95 & .50 & .17 & .00 & 100.65 \\
\hline JP-32 & $\mathrm{KO}$ & hw & & 1 & D-269106 & b & SCP & 49.20 & 16.90 & 11.40 & nd & 3.79 & 6.99 & 4.69 & 1.82 & .38 & 2.61 & 1.63 & .21 & .00 & 99.62 \\
\hline JP-33 & $\mathrm{KO}$ & hw & & 1 & D-261404 & b & SCP & 51.70 & 17.00 & 10.70 & nd & 3.27 & 5.91 & 5.28 & 2.02 & .28 & 2.21 & 1.61 & .24 & .00 & 100.22 \\
\hline JP-35 & $\mathrm{KO}$ & hw & & 1 & D-261433 & b & SCP & 50.00 & 16.70 & 11.80 & nd & 3.74 & 6.70 & 5.19 & 1.89 & .08 & 2.54 & 1.86 & .24 & .00 & 100.74 \\
\hline JP-36 & $\mathrm{KO}$ & pl & & 1 & D-269126 & b & SCP & 46.30 & 15.70 & 13.30 & nd & 6.13 & 10.70 & 2.69 & .82 & .85 & 3.19 & .45 & .17 & .00 & 100.30 \\
\hline JP-37 & $\mathrm{KO}$ & hw & & 1 & D-261419 & b & $\mathrm{SCP}$ & 48.10 & 17.20 & 12.80 & nd & 4.16 & 6.93 & 4.17 & 1.68 & .72 & 3.05 & 1.49 & .21 & .00 & 100.51 \\
\hline JP-38 & $\mathrm{KO}$ & hw & & 1 & D-261432 & $\mathrm{b}$ & SCP & 48.50 & 16.30 & 12.50 & nd & 3.97 & 6.86 & 4.61 & 1.76 & .91 & 2.76 & 2.00 & .24 & .01 & 100.42 \\
\hline JP-39 & $\mathrm{KO}$ & $\mathrm{pl}$ & & 1 & D-269116 & $\mathrm{b}$ & $\mathrm{SCP}$ & 47.40 & 14.60 & 14.60 & nd & 4.96 & 9.07 & 3.11 & 1.04 & .76 & 4.04 & .61 & .20 & .00 & 100.39 \\
\hline $\mathrm{JP}-40$ & KO & hwb & & 1 & D-261446 & b & SCP & 56.90 & 18.20 & 7.38 & nd & 1.89 & 3.60 & 6.08 & 2.55 & 1.08 & 1.51 & .68 & .25 & .00 & 100.12 \\
\hline JP-42 & $\mathrm{KO}$ & hwtd & & 1 & D-261399 & b & SCP & 58.30 & 18.10 & 6.21 & nd & 1.25 & 2.42 & 6.41 & 3.43 & 1.68 & 1.03 & .59 & .19 & .00 & 99.61 \\
\hline JP-43 & KO & hw & & 1 & D-269105 & b & SCP & 49.60 & 16.90 & 11.40 & nd & 3.67 & 7.06 & 4.88 & 1.86 & .36 & 2.63 & 1.64 & .21 & .01 & 100.22 \\
\hline JP-44 & KO & $\mathrm{pl}$ & & 1 & D-261421 & $b$ & SCP & 46.00 & 15.30 & 15.40 & nd & 6.27 & 10.50 & 2.72 & .43 & .28 & 3.50 & .46 & .20 & .00 & 101.06 \\
\hline JP-45 & $\mathrm{KO}$ & hw & & 1 & D-269104 & $b$ & SCP & 45.40 & 18.60 & 13.10 & nd & 3.38 & 4.99 & 3.30 & 1.80 & 4.35 & 2.95 & 1.81 & .26 & .00 & 99.94 \\
\hline JP-46 & $\mathrm{KO}$ & $\mathrm{pl}$ & & 1 & D-269113 & $\mathrm{b}$ & SCP & 45.30 & 16.60 & 12.90 & nd & 7.20 & 10.10 & 2.42 & .66 & 2.28 & 2.53 & .34 & .18 & .00 & 100.51 \\
\hline JP-47 & $\mathrm{KO}$ & hw & & 1 & D-269157 & b & SCP & 49.10 & 16.60 & 12.30 & nd & 4.01 & 7.06 & 4.70 & 1.81 & .00 & 2.77 & 1.68 & .22 & .00 & 100.25 \\
\hline JP-48 & $\mathrm{KO}$ & hw & & 1 & D-261435 & $\mathrm{b}$ & SCP & 48.20 & 17.30 & 12.60 & nd & 3.68 & 6.15 & 4.32 & 1.83 & 1.52 & 2.68 & 1.94 & .25 & .00 & 100.47 \\
\hline JP-49 & $\mathrm{KO}$ & $\mathrm{pl}$ & & 1 & D-269174 & b & SCP & 47.90 & 14.10 & 14.10 & nd & 5.38 & 10.00 & 3.00 & 1.04 & .47 & 3.80 & .61 & .19 & .00 & 100.59 \\
\hline JP-50 & KO & hw & & 1 & D-269162 & b & SCP & 46.40 & 17.10 & 12.70 & nd & 3.94 & 6.05 & 3.93 & 1.78 & 3.10 & 2.80 & 2.11 & .25 & .04 & 100.20 \\
\hline JP-51 & $\mathrm{KO}$ & hw & 1 & 1 & D-261401 & b & $\mathrm{SCP}$ & 47.30 & 15.90 & 13.30 & nd & 4.65 & 7.53 & 4.70 & 1.58 & .24 & 3.04 & 2.29 & .23 & .03 & 100.79 \\
\hline JP-52 & $\mathrm{KO}$ & $\mathrm{pl}$ & & 1 & D-269179 & b & SCP & 48.30 & 14.50 & 13.90 & nd & 5.32 & 9.96 & 3.16 & 1.08 & .50 & 3.75 & .60 & .19 & .00 & 101.26 \\
\hline JP-53 & KO & $\mathrm{pl}$ & & 1 & D-269173 & b & SCP & 49.00 & 15.70 & 12.80 & nd & 4.85 & 9.69 & 3.12 & 1.03 & .44 & 3.34 & .58 & .18 & .00 & 100.73 \\
\hline JP-54 & KO & hwbd & & 1 & D-261445 & b & SCP & 55.70 & 18.20 & 7.40 & nd & 2.05 & 3.23 & 6.06 & 2.61 & 2.44 & 1.49 & .64 & .28 & .02 & 100.12 \\
\hline
\end{tabular}


Table 1.-Chemical analyses-Continued

\begin{tabular}{|c|c|c|c|c|c|c|c|c|c|c|c|c|c|c|c|c|c|c|c|c|}
\hline $\begin{array}{c}\text { Sample } \\
\text { locality } \\
\text { No. }\end{array}$ & $\begin{array}{l}\text { Vol- } \\
\text { cano }\end{array}$ & Unit & $\begin{array}{c}\text { Map } \\
\text { sheet } \\
\text { No. }\end{array}$ & $\begin{array}{l}\text { Laboratory } \\
\text { No. }\end{array}$ & Remark & Collector & $\mathrm{SiO}_{2}$ & $\mathrm{Al}_{2} \mathrm{O}_{3}$ & $\mathrm{Fe}_{2} \mathrm{O}_{3}$ & $\mathrm{FeO}$ & $\mathrm{MgO}$ & $\mathrm{CaO}$ & $\mathrm{Na}_{2} \mathrm{O}$ & $\mathrm{K}_{2} \mathrm{O}$ & $\mathrm{H}_{2} \mathrm{O}$ & $\mathrm{TiO}_{2}$ & $\mathrm{P}_{2} \mathrm{O}_{5}$ & $\mathrm{MnO}$ & $\mathrm{CO}_{2}$ & Total \\
\hline JP-55 & $\mathrm{KO}$ & hw & 1 & D-261437 & b & $\mathrm{SCP}$ & 48.40 & 18.30 & 10.90 & nd & 3.20 & 4.25 & 4.71 & 2.08 & 4.56 & 2.18 & 1.49 & .27 & .04 & 100.38 \\
\hline JP-57 & $\mathrm{KO}$ & hwd & 1 & D-261405 & $b$ & SCP & 49.20 & 17.80 & 11.00 & nd & 2.88 & 5.01 & 5.07 & 1.99 & 3.03 & 2.26 & 1.57 & .24 & .01 & 100.06 \\
\hline JP-59 & $\mathrm{KO}$ & $\mathrm{pl}$ & 1 & D-269175 & $b$ & SCP & 47.70 & 14.90 & 13.60 & nd & 5.77 & 9.98 & 3.18 & 1.25 & .39 & 3.53 & .65 & .19 & .00 & 101.14 \\
\hline$J P-60$ & $\mathrm{KO}$ & hw & 1 & D-269148 & $b$ & $\mathrm{SCP}$ & 49.30 & 17.10 & 11.60 & nd & 3.59 & 7.11 & 4.54 & 1.79 & .56 & 2.83 & 1.70 & .22 & .04 & 100.38 \\
\hline JP-62 & $\mathrm{KO}$ & hw & 1 & D-261394 & b & $\mathrm{SCP}$ & 52.00 & 17.00 & 10.50 & nd & 3.22 & 5.75 & 5.45 & 2.08 & .24 & 2.12 & 1.50 & .24 & .02 & 100.12 \\
\hline JP-63 & $\mathrm{KO}$ & hwc & 1 & D-261407 & b & SCP & 51.80 & 16.80 & 10.60 & nd & 3.22 & 5.79 & 5.47 & 2.05 & .19 & 2.16 & 1.52 & .24 & .00 & 99.84 \\
\hline JP-64 & $\mathrm{KO}$ & $\mathrm{pl}$ & 1 & D-261443 & b & SCP & 47.90 & 17.60 & 12.40 & nd & 4.08 & 9.23 & 2.96 & .73 & 1.74 & 3.27 & .56 & .16 & .00 & 100.63 \\
\hline$J P-65$ & KO & hw & 1 & D-261396 & b & SCP & 52.00 & 16.80 & 10.50 & nd & 3.30 & 5.72 & 5.54 & 2.04 & .18 & 2.12 & 1.52 & .25 & .01 & 99.98 \\
\hline JP-67 & KO & hwb & 1 & D-261438 & b & $\mathrm{SCP}$ & 55.00 & 17.60 & 9.56 & nd & 2.27 & 4.40 & 5.47 & 2.75 & .23 & 1.46 & 1.03 & .24 & .01 & 100.02 \\
\hline JP-68 & $\mathrm{KO}$ & hw & 1 & D-269158 & b & SCP & 49.10 & 17.00 & 11.20 & nd & 3.59 & 5.94 & 4.67 & 1.90 & 1.91 & 2.41 & 1.70 & .24 & .01 & 99.67 \\
\hline JP-69 & $\mathrm{KO}$ & hw & 1 & D-261422 & b & SCP & 50.90 & 17.80 & 10.70 & nd & 3.03 & 5.22 & 4.84 & 1.99 & 1.21 & 2.29 & 1.60 & .23 & .00 & 99.81 \\
\hline JP-70 & $\mathrm{KO}$ & $\mathrm{pl}$ & 1 & D-269118 & $b$ & SCP & 47.50 & 15.50 & 13.10 & nd & 6.74 & 9.07 & 3.05 & 1.12 & .81 & 2.87 & .47 & .18 & .00 & 100.41 \\
\hline JP-71 & $\mathrm{KO}$ & hw & 1 & D-261420 & $\mathrm{b}$ & SCP & 46.30 & 17.70 & 13.30 & nd & 3.96 & 5.59 & 3.68 & 1.67 & 3.40 & 3.08 & 1.57 & .22 & .01 & 100.48 \\
\hline JP-72 & $\mathrm{KO}$ & $\mathrm{pl}$ & 1 & D-261439 & b & SCP & 48.10 & 15.40 & 12.80 & nd & 6.85 & 9.56 & 3.23 & 1.16 & .00 & 2.82 & .50 & .18 & .00 & 100.60 \\
\hline JP-74 & KO & pl & 1 & D-261440 & $b$ & SCP & 48.80 & 14.30 & 13.80 & nd & 5.53 & 10.40 & 3.24 & 1.11 & .00 & 3.73 & .58 & .19 & .00 & 101.68 \\
\hline JP-75 & $\mathrm{KO}$ & hw & 1 & D-269159 & b & SCP & 52.10 & 17.90 & 9.69 & nd & 2.46 & 4.81 & 4.82 & 2.22 & 1.83 & 2.27 & 1.04 & .26 & .00 & 99.40 \\
\hline JP-76 & KO & $\mathrm{pl}$ & 1 & D-269178 & b & SCP & 47.10 & 15.90 & 14.10 & nd & 5.02 & 11.00 & 2.83 & .54 & .30 & 3.40 & .44 & .19 & .00 & 100.82 \\
\hline JP-77 & $\mathrm{KO}$ & hw & 1 & D-261395 & b & SCP & 52.00 & 16.90 & 10.60 & nd & 3.27 & 5.84 & 5.59 & 2.09 & .20 & 2.15 & 1.50 & .24 & .05 & 100.43 \\
\hline JP-79 & $\mathrm{KO}$ & hw & 1 & D-261408 & b & SCP & 52.00 & 17.00 & 10.70 & nd & 3.30 & 5.78 & 5.68 & 2.06 & .00 & 2.18 & 1.53 & .25 & .00 & 100.48 \\
\hline JP-80 & $\mathrm{KO}$ & hw & 1 & D-277482 & b & $\mathrm{SCP}$ & 53.00 & 17.40 & 9.33 & nd & 2.83 & 5.50 & 5.38 & 2.16 & .48 & 2.20 & 1.04 & .23 & nd & 99.55 \\
\hline JP-83 & $\mathrm{KO}$ & hw & 1 & D-269109 & b & SCP & 47.20 & 16.90 & 12.60 & nd & 3.81 & 6.32 & 4.45 & 1.79 & 2.36 & 2.69 & 1.96 & .24 & .02 & 100.34 \\
\hline JP-84 & $\mathrm{KO}$ & $\mathrm{pl}$ & 1 & D-269140 & $\mathrm{b}$ & SCP & 48.40 & 15.30 & 12.70 & nd & 6.94 & 9.77 & 3.30 & 1.16 & .00 & 2.81 & .48 & .18 & .00 & 101.04 \\
\hline JP-85 & $\mathrm{KO}$ & pl & 1 & D-269176 & b & SCP & 48.10 & 14.90 & 13.00 & nd & 6.12 & 10.50 & 2.85 & 1.01 & .87 & 3.34 & .52 & .18 & .08 & 101.47 \\
\hline JP-86 & $\mathrm{KO}$ & $\mathrm{pl}$ & 1 & D-261441 & b & $\mathrm{SCP}$ & 48.00 & 14.90 & 13.70 & nd & 5.03 & 9.83 & 3.31 & 1.22 & .44 & 3.33 & .52 & .19 & .00 & 100.47 \\
\hline JP-87 & $\mathrm{KO}$ & hw & 1 & D-269123 & b & SCP & 51.80 & 17.00 & 10.60 & nd & 3.19 & 5.75 & 5.40 & 2.04 & .14 & 2.12 & 1.50 & .26 & .00 & 99.80 \\
\hline JP-88 & $\mathrm{KO}$ & $\mathrm{pl}$ & 1 & D-269177 & b & SCP & 48.50 & 15.20 & 14.40 & nd & 4.61 & 10.20 & 2.93 & .88 & .32 & 3.57 & .47 & .19 & .00 & 101.27 \\
\hline JP-89 & $\mathrm{KO}$ & hw & 1 & D-269143 & $b$ & SCP & 49.70 & 17.40 & 11.20 & nd & 3.17 & 5.39 & 4.65 & 1.91 & 1.50 & 2.34 & 1.68 & .26 & .00 & 99.20 \\
\hline JP-90 & $\mathrm{KO}$ & hwc & 1 & D-269112 & $b$ & SCP & 43.20 & 17.80 & 13.80 & nd & 4.01 & 6.04 & 3.64 & 1.50 & 4.63 & 2.99 & 2.27 & .26 & .02 & 100.16 \\
\hline JP-91 & KO & hw & 1 & D-269110 & b & SCP & 49.80 & 17.70 & 11.40 & nd & 2.84 & 5.28 & 4.71 & 1.91 & 1.89 & 2.29 & 1.62 & .23 & .00 & 99.67 \\
\hline JP-92 & $\mathrm{KO}$ & hw & 1 & D-269144 & b & SCP & 51.40 & 17.00 & 10.60 & nd & 3.37 & 6.00 & 5.13 & 1.94 & .65 & 2.29 & 1.66 & .24 & .03 & 100.31 \\
\hline JP-93 & $\mathrm{KO}$ & hwbd & 1 & D-269147 & $b$ & SCP & 53.60 & 18.10 & 8.53 & nd & 2.36 & 3.59 & 5.98 & 2.43 & 2.17 & 1.54 & 1.05 & .25 & .00 & 99.60 \\
\hline JP-94 & $\mathrm{KO}$ & $\mathrm{pl}$ & 1 & D-269172 & b & SCP & 46.50 & 15.60 & 13.40 & nd & 7.07 & 9.41 & 2.72 & 1.00 & 1.29 & 2.98 & .50 & .18 & .00 & 100.65 \\
\hline JP-95 & $\mathrm{KO}$ & $\mathrm{pl}$ & 1 & D-277478 & b & SCP & 46.70 & 15.00 & 13.00 & nd & 6.76 & 11.60 & 2.64 & .51 & .34 & 3.02 & .40 & .17 & nd & 100.14 \\
\hline JP-96 & $\mathrm{KO}$ & $\mathrm{pl}$ & 1 & D-261442 & $b$ & SCP & 47.90 & 14.00 & 14.90 & nd & 5.75 & 10.70 & 2.85 & .73 & .00 & 3.51 & .46 & .20 & .00 & 101.00 \\
\hline JP-97 & $\mathrm{KO}$ & hw & 1 & D-269111 & b & SCP & 47.50 & 18.00 & 11.20 & nd & 3.18 & 5.01 & 4.80 & 1.89 & 3.95 & 2.34 & 1.68 & .25 & .03 & 99.83 \\
\hline JP-99 & $\mathrm{KO}$ & hw & 1 & D-269108 & $b$ & SCP & 46.10 & 16.80 & 12.90 & nd & 3.90 & 6.56 & 4.29 & 1.69 & 2.83 & 2.78 & 2.10 & .24 & .02 & 100.21 \\
\hline JP-101 & $\mathrm{KO}$ & hw & 1 & D-269165 & $b$ & SCP & 48.30 & 17.70 & 11.30 & nd & 3.62 & 4.87 & 4.48 & 1.87 & 3.31 & 2.38 & 1.66 & .25 & .03 & 99.77 \\
\hline JP-103 & $\mathrm{KO}$ & $\mathrm{pl}$ & 1 & D-269141 & $b$ & $\mathrm{SCP}$ & 48.00 & 15.20 & 12.90 & nd & 5.31 & 9.53 & 3.49 & 1.28 & .85 & 3.20 & .66 & .18 & nd & 100.60 \\
\hline JP-104 & $\mathrm{KO}$ & hw & 1 & D-269107 & $b$ & SCP & 50.00 & 18.60 & 10.30 & nd & 3.05 & 3.85 & 4.14 & 2.16 & 3.56 & 2.35 & 1.10 & .28 & .00 & 99.39 \\
\hline JP-105 & $\mathrm{KO}$ & $\mathrm{pl}$ & 1 & D-269171 & $b$ & SCP & 45.10 & 16.10 & 12.90 & nd & 8.33 & 9.00 & 1.98 & .70 & 3.60 & 2.59 & .38 & .18 & .00 & 100.86 \\
\hline
\end{tabular}


Table 1.-Chemical analyses-Continued

\begin{tabular}{|c|c|c|c|c|c|c|c|c|c|c|c|c|c|c|c|c|c|c|c|c|}
\hline $\begin{array}{l}\text { Sample } \\
\text { locality } \\
\text { No. }\end{array}$ & $\begin{array}{l}\text { Vol- } \\
\text { cano }\end{array}$ & Unit & $\begin{array}{l}\text { Map } \\
\text { sheet } \\
\text { No. }\end{array}$ & $\begin{array}{c}\text { Laboratory } \\
\text { No. }\end{array}$ & Remark & Collector & $\mathrm{SiO}_{2}$ & $\mathrm{Al}_{2} \mathrm{O}_{3}$ & $\mathrm{Fe}_{2} \mathrm{O}_{3}$ & $\mathrm{FeO}$ & $\mathrm{MgO}$ & $\mathrm{CaO}$ & $\mathrm{Na}_{2} \mathrm{O}$ & $\mathrm{K}_{2} \mathrm{O}$ & $\mathrm{H}_{2} \mathrm{O}$ & $\mathrm{TO}_{2}$ & $\mathrm{P}_{2} \mathrm{O}_{5}$ & $\mathrm{MnO}$ & $\mathrm{CO}_{2}$ & Total \\
\hline JP-106 & $\mathrm{KO}$ & hw & 1 & D-269166 & $\mathrm{b}$ & $\overline{S C P}$ & 51.40 & 17.30 & 10.90 & nd & 3.27 & 5.51 & 4.99 & 2.02 & .43 & 2.20 & 1.54 & .26 & .00 & 99.82 \\
\hline JP-107 & $\mathrm{KO}$ & $\mathrm{pl}$ & 1 & D-269142 & b & SCP & 47.70 & 15.00 & 14.00 & nd & 5.03 & 9.46 & 3.18 & 1.32 & .96 & 3.40 & .54 & .19 & .00 & 100.78 \\
\hline JP-109 & $\mathrm{KO}$ & hw & 1 & D-269145 & b & SCP & 50.90 & 16.70 & 11.20 & nd & 3.63 & 6.00 & 5.04 & 1.94 & .46 & 2.26 & 1.62 & .27 & .08 & 100.10 \\
\hline JP-110 & $\mathrm{KO}$ & $\mathrm{pl}$ & 1 & D-277477 & b & SCP & 47.90 & 16.30 & 13.50 & nd & 4.50 & 10.40 & 2.87 & .75 & .16 & 3.28 & .44 & .18 & nd & 100.28 \\
\hline $\mathrm{JP}-111$ & $\mathrm{KO}$ & pl & 1 & D-277476 & b & SCP & 46.20 & 15.00 & 13.20 & nd & 6.78 & 11.20 & 2.46 & .54 & .79 & 3.03 & .40 & .17 & nd & 99.77 \\
\hline JP-112 & $\mathrm{KO}$ & hw & 1 & D-269146 & b & SCP & 47.60 & 18.00 & 12.00 & nd & 3.55 & 5.02 & 4.38 & 1.82 & 3.71 & 2.47 & 1.80 & .25 & .04 & 100.64 \\
\hline JP-114 & $\mathrm{KO}$ & $\mathrm{pl}$ & 1 & D-269138 & b & SCP & 48.90 & 15.90 & 13.00 & nd & 4.07 & 8.33 & 3.45 & 1.26 & 1.04 & 3.30 & .68 & .20 & .00 & 100.13 \\
\hline JP-115 & KO & hw & 1 & D-269149 & $b$ & $\mathrm{SCP}$ & 51.20 & 19.30 & 9.69 & nd & 2.16 & 3.53 & 4.75 & 2.27 & 2.78 & 1.85 & 1.24 & .25 & .00 & 99.02 \\
\hline JP-116 & $\mathrm{KO}$ & $\mathrm{pl}$ & 1 & D-283178 & b & SCP & 49.10 & 16.60 & 11.70 & nd & 6.41 & 11.10 & 2.45 & .33 & .44 & 2.30 & .23 & .15 & nd & 100.81 \\
\hline JP-117 & $\mathrm{KO}$ & $\mathrm{pl}$ & 1 & D-277487 & b & SCP & 50.30 & 13.70 & 12.20 & nd & 7.36 & 11.60 & 2.03 & .15 & .44 & 2.37 & .22 & .17 & nd & 100.54 \\
\hline JP-118 & $\mathrm{KO}$ & $\mathrm{pl}$ & 1 & D-283180 & b & SCP & 49.70 & 14.20 & 13.20 & nd & 6.54 & 11.00 & 2.28 & .21 & .28 & 2.77 & .28 & .19 & nd & 100.65 \\
\hline JP-119 & $\mathrm{KO}$ & $\mathrm{pl}$ & 1 & D-283179 & b & SCP & 51.20 & 13.60 & 11.90 & nd & 7.32 & 11.30 & 2.07 & .40 & .12 & 2.20 & .22 & .17 & nd & 100.50 \\
\hline$J P-120$ & $\mathrm{KO}$ & hw & 1 & D-269150 & b & SCP & 52.50 & 18.30 & 9.28 & nd & 2.46 & 3.85 & 5.43 & 2.28 & 2.49 & 1.78 & 1.20 & .26 & .02 & 99.85 \\
\hline JP-122 & $\mathrm{KO}$ & $\mathrm{pl}$ & 1 & D-277486 & b & SCP & 51.80 & 13.90 & 12.40 & nd & 7.14 & 11.30 & 2.31 & .18 & .00 & 1.87 & .17 & .19 & nd & 101.26 \\
\hline JP-123 & $\mathrm{KO}$ & hw & 1 & D-269151 & b & $\mathrm{SCP}$ & 52.10 & 18.40 & 9.57 & nd & 2.55 & 3.79 & 5.34 & 2.25 & 2.72 & 1.83 & 1.22 & .25 & .03 & 100.05 \\
\hline JP-124 & $\mathrm{KO}$ & hwb & 1 & D-269132 & b & SCP & 55.40 & 17.90 & 8.03 & nd & 1.94 & 3.29 & 5.66 & 3.13 & 1.94 & 1.55 & .86 & .18 & .00 & 99.88 \\
\hline JP-126 & $\mathrm{KO}$ & hwb & 1 & D-269131 & b & SCP & 55.40 & 18.10 & 7.95 & nd & 1.82 & 3.13 & 5.60 & 3.17 & 1.44 & 1.49 & .86 & .20 & .00 & 99.16 \\
\hline JP-128 & $\mathrm{KO}$ & hwb & 1 & D-269128 & b & $\mathrm{SCP}$ & 55.50 & 17.70 & 8.13 & nd & 1.69 & 3.79 & 5.70 & 2.94 & .80 & 1.56 & .95 & .21 & .00 & 98.97 \\
\hline JP-130 & $\mathrm{KO}$ & hwbd & 1 & D-269127 & b & SCP & 55.60 & 18.90 & 6.84 & nd & 1.14 & 2.15 & 6.26 & 2.98 & 3.65 & .92 & .38 & .27 & .02 & 99.11 \\
\hline JP-131 & $\mathrm{KO}$ & hw & 1 & D-277464 & $b$ & SCP & 49.60 & 15.30 & 12.40 & nd & 4.13 & 8.62 & 3.57 & 1.36 & .99 & 3.09 & .69 & .19 & nd & 99.94 \\
\hline JP-132 & $\mathrm{KO}$ & hwb & 1 & D-277465 & b & SCP & 55.40 & 17.10 & 8.43 & nd & 2.30 & 4.19 & 5.80 & 2.96 & .38 & 1.64 & 1.03 & .19 & nd & 99.42 \\
\hline JP-133 & $\mathrm{KO}$ & hwb & 1 & D-277466 & b & $\mathrm{SCP}$ & 56.60 & 17.60 & 7.29 & nd & 1.78 & 2.96 & 5.87 & 3.39 & 1.55 & 1.31 & .80 & .20 & nd & 99.35 \\
\hline JP-134 & $\mathrm{KO}$ & hw & 1 & D-277462 & b & $\mathrm{SCP}$ & 47.80 & 17.30 & 12.50 & nd & 3.33 & 6.09 & 4.30 & 1.92 & 1.54 & 2.77 & 1.92 & .25 & nd & 99.72 \\
\hline JP-135 & $\mathrm{KO}$ & hw & 1 & D-277463 & $b$ & $\mathrm{SCP}$ & 50.10 & 16.90 & 11.20 & nd & 3.31 & 6.25 & 4.93 & 2.03 & .39 & 2.40 & 1.69 & .23 & nd & 99.43 \\
\hline$J Q-1$ & MK & $\mathrm{hm}$ & 1 & D-260179 & b & WSW & 45.70 & 13.90 & 14.70 & nd & 7.68 & 10.60 & 2.19 & .50 & .86 & 3.48 & .43 & .20 & .00 & 100.24 \\
\hline $\mathrm{JQ}-2$ & MK & $\mathrm{hm}$ & 1 & D-260178 & b & WSW & 45.50 & 11.50 & 13.10 & nd & 13.50 & 10.60 & 1.76 & .55 & .00 & 2.54 & .31 & .17 & .00 & 99.53 \\
\hline $\mathrm{JQ}-3$ & MK & $\mathrm{hm}$ & 1 & D-260180 & b & WSW & 47.10 & 12.90 & 13.70 & nd & 9.29 & 11.30 & 2.24 & .56 & .00 & 3.00 & .38 & .18 & .00 & 100.65 \\
\hline $\mathrm{JQ}-4$ & MK & lb & 1 & D-260173 & $b$ & WSW & 54.70 & 18.10 & 8.99 & nd & 2.25 & 3.82 & 5.25 & 2.75 & .96 & 1.25 & .88 & .21 & .00 & 99.16 \\
\hline$J Q-5$ & MK & $\mathrm{hm}$ & 1 & D-260181 & $b$ & WSW & 46.30 & 12.60 & 13.20 & nd & 11.50 & 11.10 & 2.02 & .54 & .00 & 2.58 & .32 & .18 & .00 & 100.34 \\
\hline JQ-6 & MK & lb & 1 & D-260191 & $\mathrm{b}$ & WSW & 54.70 & 17.40 & 9.49 & nd & 2.20 & 4.62 & 5.42 & 2.67 & .34 & 1.49 & 1.06 & .24 & .00 & 99.63 \\
\hline JQ-7 & $\mathrm{KO}$ & hwc & 1 & D-261413 & $b$ & $\mathrm{SCP}$ & 45.90 & 17.10 & 12.60 & nd & 4.02 & 6.13 & 3.92 & 1.60 & 4.05 & 2.78 & 2.01 & .25 & .02 & 100.38 \\
\hline JQ-8 & MK & $\mathrm{hm}$ & 1 & D-260172 & $\mathrm{b}$ & WSW & 46.20 & 12.70 & 13.50 & nd & 10.40 & 10.80 & 2.20 & .73 & .60 & 2.91 & .37 & .18 & .00 & 100.59 \\
\hline JQ-9 & MK & $\mathrm{hm}$ & 1 & D-260171 & b & WSW & 45.90 & 14.80 & 14.20 & nd & 6.61 & 10.90 & 2.36 & .56 & .99 & 3.44 & .43 & .19 & .00 & 100.38 \\
\hline JQ-10 & MK & lb & 1 & D-260175 & b & WSW & 54.90 & 17.40 & 9.17 & nd & 2.22 & 4.46 & 5.60 & 2.74 & .00 & 1.39 & .98 & .23 & .00 & 99.09 \\
\hline JQ-11 & KO & hw & 1 & D-269121 & $\mathrm{b}$ & SCP & 46.30 & 17.10 & 13.10 & nd & 3.39 & 6.65 & 4.11 & 1.78 & 2.00 & 2.85 & 2.16 & .25 & .00 & 99.69 \\
\hline JQ-12 & $\mathrm{KO}$ & hw & 1 & D-269120 & $\mathrm{b}$ & $\mathrm{SCP}$ & 48.30 & 16.50 & 12.30 & nd & 3.99 & 7.21 & 4.77 & 1.71 & .00 & 2.73 & 2.04 & .22 & .00 & 99.77 \\
\hline JQ-13 & MK & $\mathrm{hm}$ & 1 & D-260174 & $b$ & WSW & 46.10 & 10.70 & 12.60 & nd & 15.40 & 10.60 & 1.74 & .51 & .10 & 2.06 & .25 & .17 & .00 & 100.23 \\
\hline JQ-14 & $\mathrm{KO}$ & pld & 1 & D-269119 & b & $\mathrm{SCP}$ & 48.40 & 16.10 & 12.10 & nd & 5.04 & 10.40 & 3.31 & 1.11 & .00 & 3.30 & .60 & .17 & .00 & 100.53 \\
\hline JQ-15 & $\mathrm{KO}$ & hw & 1 & D-277475 & $\mathrm{b}$ & SCP & 48.20 & 16.40 & 12.30 & nd & 4.08 & 7.27 & 4.83 & 1.70 & .14 & 2.78 & 2.05 & .22 & nd & 99.97 \\
\hline JQ-16 & MK & $\mathrm{hm}$ & 1 & D-260192 & b & WSW & 46.10 & 12.70 & 13.60 & nd & 9.79 & 11.00 & 2.26 & .75 & .26 & 2.99 & .39 & .18 & .00 & 100.02 \\
\hline
\end{tabular}


Table 1.-Chemical analyses-Continued

\begin{tabular}{|c|c|c|c|c|c|c|c|c|c|c|c|c|c|c|c|c|c|c|c|c|}
\hline $\begin{array}{c}\text { Sample } \\
\text { locality } \\
\text { No. }\end{array}$ & $\begin{array}{l}\text { Vol- } \\
\text { cano }\end{array}$ & Unit & $\begin{array}{c}\text { Map } \\
\text { sheet } \\
\text { No. }\end{array}$ & $\begin{array}{l}\text { Laboratory } \\
\text { No. }\end{array}$ & Remark & Collector & $\mathrm{SiO}_{2}$ & $\mathrm{Al}_{2} \mathrm{O}_{3}$ & $\mathrm{Fe}_{2} \mathrm{O}_{3}$ & $\mathrm{FeO}$ & $\mathrm{MgO}$ & $\mathrm{CaO}$ & $\mathrm{Na}_{2} \mathrm{O}$ & $\mathrm{K}_{2} \mathrm{O}$ & $\mathrm{H}_{2} \mathrm{O}$ & $\mathrm{TiO}_{2}$ & $\mathrm{P}_{2} \mathrm{O}_{5}$ & $\mathrm{MnO}$ & $\mathrm{CO}_{2}$ & Total \\
\hline JQ-17 & MK & $\mathrm{hm}$ & 1 & D-260184 & $\bar{b}$ & WSW & 45.10 & 11.00 & 13.10 & nd & 14.50 & 10.60 & 1.72 & .43 & .73 & 2.45 & .29 & .17 & .00 & 100.09 \\
\hline JQ-18 & KO & $\mathrm{pl}$ & 1 & D-277480 & b & SCP & 47.90 & 15.10 & 14.00 & nd & 7.46 & 10.30 & 2.57 & .42 & .03 & 2.72 & .29 & .18 & nd & 100.97 \\
\hline JQ-19 & KO & hw & 1 & D-277474 & $\mathrm{b}$ & SCP & 48.80 & 16.50 & 11.80 & nd & 3.58 & 7.08 & 4.89 & 1.76 & .32 & 2.68 & 2.00 & .22 & nd & 99.63 \\
\hline JQ-20 & MK & $\mathrm{hm}$ & 1 & D-260190 & b & WSW & 46.90 & 13.50 & 13.80 & nd & 7.97 & 11.20 & 2.59 & .84 & .09 & 3.23 & .42 & .19 & .00 & 100.73 \\
\hline JQ-21 & MK & $\mathrm{hm}$ & 1 & D-260182 & b & WSW & 45.30 & 14.70 & 13.60 & nd & 6.74 & 11.20 & 2.27 & .57 & 1.52 & 3.42 & .41 & .18 & .00 & 99.91 \\
\hline$J Q-22$ & MK & $\mathrm{hm}$ & 1 & D-277473 & b & SCP & 44.90 & 12.40 & 13.80 & nd & 11.90 & 10.20 & 1.68 & .55 & 1.59 & 2.92 & .34 & .19 & nd & 100.47 \\
\hline JQ-23 & MK & $\mathrm{hm}$ & 1 & D-277460 & b & $\mathrm{SCP}$ & 46.40 & 11.70 & 13.10 & nd & 12.20 & 11.00 & 1.93 & .62 & .71 & 2.73 & .33 & .17 & nd & 100.89 \\
\hline JQ-24 & $\mathrm{KO}$ & hw & 1 & D-277472 & $b$ & $\mathrm{SCP}$ & 47.80 & 16.40 & 12.50 & nd & 3.90 & 7.07 & 4.58 & 1.73 & .55 & 2.73 & 2.04 & .23 & nd & 99.53 \\
\hline JQ-25 & MK & $\mathrm{hm}$ & 1 & D-260183 & $b$ & WSW & 46.40 & 11.80 & 12.60 & nd & 13.30 & 11.10 & 1.93 & .52 & .04 & 2.26 & .26 & .17 & .00 & 100.38 \\
\hline JR-1 & MK & $\mathrm{hm}$ & 1 & D-271452 & b & WSW & 46.80 & 14.40 & 14.20 & nd & 6.20 & 11.40 & 2.73 & .78 & .40 & 3.52 & .44 & .19 & .00 & 101.06 \\
\hline $\mathrm{JR}-2$ & MK & $\mathrm{hm}$ & 1 & D-271451 & $b$ & WSW & 46.70 & 13.90 & 15.30 & nd & 5.26 & 9.36 & 3.26 & 1.23 & .68 & 4.33 & .65 & .21 & .00 & 100.88 \\
\hline JR-3 & MK & $\mathrm{hm}$ & 1 & D-271454 & $b$ & WSW & 47.30 & 14.40 & 13.40 & nd & 6.80 & 11.80 & 2.49 & .75 & .18 & 3.41 & .43 & .18 & .00 & 101.14 \\
\hline JR-4 & MK & $\mathrm{hm}$ & 1 & D-271455 & b & WSW & 46.50 & 14.60 & 12.60 & nd & 8.84 & 12.40 & 2.01 & .27 & .97 & 2.50 & .28 & .17 & .00 & 101.14 \\
\hline JR-5 & MK & $\mathrm{hm}$ & 1 & D-271445 & b & WSW & 46.40 & 14.50 & 14.50 & nd & 6.33 & 11.30 & 2.49 & .75 & .50 & 3.56 & .42 & .19 & .00 & 100.94 \\
\hline JR-6 & MK & 1 & 1 & D-271446 & b & WSW & 48.90 & 17.10 & 12.30 & nd & 4.14 & 7.13 & 4.24 & 1.80 & .72 & 2.94 & .97 & .22 & .00 & 100.46 \\
\hline JR-7 & $\mathrm{MK}$ & $\mathrm{hm}$ & 1 & D-271447 & b & WSW & 48.40 & 13.70 & 15.30 & nd & 4.46 & 8.38 & 3.25 & 1.32 & 1.03 & 4.10 & .67 & .23 & .00 & 100.84 \\
\hline JR-8 & MK & $\mathrm{hm}$ & 1 & D-271449 & b & WSW & 45.00 & 15.00 & 16.70 & nd & 4.48 & 7.12 & 3.22 & 1.56 & 1.05 & 5.17 & .85 & .24 & .00 & 100.39 \\
\hline JR-9 & $\mathrm{MK}$ & $\mathrm{hm}$ & 1 & D-271448 & b & WSW & 47.30 & 14.00 & 14.60 & nd & 5.78 & 10.50 & 3.19 & 1.03 & .00 & 3.88 & .54 & .20 & .00 & 101.02 \\
\hline JR-10 & MK & $\mathrm{hm}$ & 1 & D-271450 & $b$ & WSW & 47.90 & 13.80 & 13.80 & nd & 6.75 & 11.20 & 2.71 & .83 & .00 & 3.34 & .42 & .18 & .00 & 100.93 \\
\hline JR-11 & MK & $\mathrm{hm}$ & 1 & D-271453 & $b$ & WSW & 46.90 & 13.20 & 14.90 & nd & 7.50 & 9.92 & 2.98 & 1.06 & .01 & 3.82 & .55 & .20 & .00 & 101.04 \\
\hline JR-12 & MK & $\mathrm{hm}$ & 1 & D-271456 & b & WSW & 47.60 & 14.20 & 14.70 & nd & 6.07 & 10.60 & 2.93 & .80 & .18 & 3.75 & .52 & .21 & .00 & 101.56 \\
\hline JR-13 & MK & $\mathrm{hm}$ & 1 & D-260189 & b & WSW & 46.20 & 14.70 & 15.10 & nd & 5.24 & 9.58 & 2.59 & .85 & .93 & 3.62 & .52 & .21 & .00 & 99.54 \\
\hline JS-1 & MK & $\mathrm{hm}$ & 2 & D-271466 & $b$ & WSW & 46.50 & 13.70 & 15.20 & nd & 5.27 & 9.78 & 3.26 & 1.30 & .00 & 4.88 & .71 & .20 & .00 & 100.80 \\
\hline JS-2 & MK & $\mathrm{hm}$ & 2 & D-271457 & $b$ & WSW & 46.50 & 13.70 & 15.40 & nd & 5.15 & 9.54 & 3.15 & 1.34 & .25 & 5.00 & .75 & .21 & .00 & 100.99 \\
\hline JS-3 & MK & $\mathrm{hm}$ & 2 & D-271463 & $b$ & WSW & 45.90 & 13.30 & 14.90 & nd & 5.40 & 9.51 & 3.08 & 1.30 & .92 & 4.79 & .73 & .20 & .00 & 100.03 \\
\hline JS-4 & MK & $\mathrm{hm}$ & 2 & D-271464 & b & WSW & 46.60 & 14.80 & 14.30 & nd & 6.17 & 10.50 & 2.70 & .66 & .76 & 3.75 & .34 & .19 & .00 & 100.77 \\
\hline JS-5 & MK & $\mathrm{hm}$ & 2 & D-271465 & b & WSW & 46.60 & 12.90 & 14.60 & nd & 8.23 & 10.00 & 2.94 & 1.00 & .67 & 3.54 & .52 & .19 & .00 & 101.19 \\
\hline JS-6 & MK & $\mathrm{hm}$ & 2 & D-271462 & $b$ & WSW & 47.40 & 12.70 & 13.80 & nd & 9.52 & 11.00 & 2.66 & .86 & .00 & 3.22 & .44 & .18 & .00 & 101.78 \\
\hline JS-7 & MK & $\mathrm{hm}$ & 2 & D-271461 & $b$ & WSW & 48.60 & 13.40 & 13.30 & nd & 7.89 & 11.30 & 2.37 & .51 & .13 & 3.08 & .34 & .18 & .00 & 101.10 \\
\hline JS-8 & MK & $\mathrm{hm}$ & 2 & D-271459 & $b$ & WSW & 46.70 & 13.70 & 14.50 & nd & 7.25 & 9.64 & 3.53 & 1.22 & .00 & 3.83 & .64 & .19 & .00 & 101.20 \\
\hline JS-9 & MK & $\mathrm{hm}$ & 2 & D-271460 & b & WSW & 47.40 & 10.60 & 12.70 & nd & 14.40 & 11.40 & 1.74 & .43 & .09 & 2.21 & .26 & .18 & .00 & 101.41 \\
\hline JS-10 & MK & $\mathrm{hm}$ & 2 & D-271458 & b & WSW & 48.90 & 13.30 & 14.30 & nd & 7.02 & 10.80 & 2.50 & .61 & .21 & 3.27 & .39 & .19 & .00 & 101.49 \\
\hline $\mathrm{KN}-1$ & $\mathrm{KO}$ & $\mathrm{pl}$ & 1 & D-283171 & $\mathrm{b}$ & MOG & 48.00 & 14.00 & 15.20 & nd & 5.22 & 10.20 & 3.02 & .85 & .00 & 3.71 & .53 & .21 & nd & 100.94 \\
\hline $\mathrm{KN}-2$ & KO & $\mathrm{pl}$ & 1 & D-269467 & b & MOG & 49.10 & 14.30 & 14.70 & nd & 4.63 & 8.47 & 3.72 & 1.38 & .00 & 4.01 & .80 & .22 & .00 & 101.33 \\
\hline $\mathrm{KN}-3$ & $\mathrm{KO}$ & pl & 1 & D-269464 & b & MOG & 47.90 & 14.80 & 13.70 & nd & 4.92 & 9.51 & 3.65 & 1.26 & .51 & 3.60 & .68 & .19 & .00 & 100.72 \\
\hline $\mathrm{KN}-4$ & $\mathrm{KO}$ & $\mathrm{pl}$ & 1 & D-269468 & b & MOG & 49.10 & 14.60 & 14.60 & nd & 4.79 & 8.53 & 3.62 & 1.37 & .00 & 4.00 & .79 & .22 & .00 & 101.62 \\
\hline $\mathrm{KN}-5$ & KO & $\mathrm{pl}$ & 1 & D-276874 & b & MOG & 48.00 & 15.00 & 13.60 & nd & 4.87 & 9.56 & 3.44 & 1.24 & .00 & 3.58 & .67 & .19 & nd & 100.15 \\
\hline $\mathrm{KN}-6$ & KO & pl & 1 & D-269480 & b & MOG & 48.00 & 14.20 & 15.10 & nd & 4.90 & 9.79 & 3.39 & 1.12 & .00 & 4.01 & .64 & .20 & .00 & 101.35 \\
\hline $\mathrm{KN}-7$ & KO & $\mathrm{pl}$ & 1 & D-269476 & $\mathrm{b}$ & MOG & 47.70 & 16.70 & 13.60 & nd & 5.16 & 7.70 & 4.09 & 1.48 & .00 & 3.72 & .87 & .19 & .00 & 101.21 \\
\hline $\mathrm{KN}-8$ & KO & $\mathrm{pl}$ & 1 & D-269471 & $b$ & MOG & 48.30 & 14.80 & 13.50 & nd & 6.15 & 10.70 & 2.83 & .77 & .14 & 3.21 & .46 & .19 & .04 & 101.09 \\
\hline
\end{tabular}


Table 1.-Chemical analyses-Continued

\begin{tabular}{|c|c|c|c|c|c|c|c|c|c|c|c|c|c|c|c|c|c|c|c|c|}
\hline $\begin{array}{l}\text { Sample } \\
\text { locality } \\
\text { No. }\end{array}$ & $\begin{array}{l}\text { Vol- } \\
\text { cano }\end{array}$ & Unit & $\begin{array}{c}\text { Map } \\
\text { sheet } \\
\text { No. }\end{array}$ & $\begin{array}{l}\text { Laboratory } \\
\text { No. }\end{array}$ & Remark & Collector & $\mathrm{SiO}_{2}$ & $\mathrm{Al}_{2} \mathrm{O}_{3}$ & $\mathrm{Fe}_{2} \mathrm{O}_{3}$ & $\mathrm{FeO}$ & $\mathrm{MgO}$ & $\mathrm{CaO}$ & $\mathrm{Na}_{2} \mathrm{O}$ & $\mathrm{K}_{2} \mathrm{O}$ & $\mathrm{H}_{2} \mathrm{O}$ & $\mathrm{TiO}_{2}$ & $\mathrm{P}_{2} \mathrm{O}_{5}$ & $\mathrm{MnO}$ & $\mathrm{CO}_{2}$ & Total \\
\hline $\mathrm{KN}-9$ & $\mathrm{KO}$ & $\mathrm{pl}$ & 1 & D-269481 & $\mathrm{b}$ & MOG & 47.70 & 14.50 & 13.00 & nd & 7.75 & 11.20 & 2.60 & .69 & .00 & 2.86 & .42 & .18 & .00 & 100.90 \\
\hline $\mathrm{KN}-10$ & KO & $\mathrm{pl}$ & 1 & D-269475 & b & MOG & 47.20 & 14.30 & 13.30 & nd & 8.22 & 11.10 & 2.52 & .64 & .00 & 2.86 & .38 & .18 & .00 & 100.70 \\
\hline $\mathrm{KN}-11$ & KO & $\mathrm{pl}$ & 1 & D-269478 & $b$ & MOG & 48.50 & 15.40 & 13.70 & nd & 5.42 & 10.80 & 2.53 & .50 & .51 & 3.11 & .40 & .19 & .00 & 101.06 \\
\hline $\mathrm{KN}-12$ & $\mathrm{KO}$ & $\mathrm{pl}$ & 1 & D-269477 & $b$ & MOG & 49.10 & 15.00 & 13.30 & nd & 5.36 & 10.50 & 2.56 & .61 & .68 & 3.02 & .37 & .18 & .00 & 100.68 \\
\hline $\mathrm{KO}-1$ & KO & hw & 1 & D-269430 & $\mathrm{b}$ & MOG & 50.20 & 17.20 & 11.30 & nd & 3.25 & 6.20 & 4.90 & 1.89 & .39 & 2.34 & 1.75 & .26 & .00 & 99.68 \\
\hline $\mathrm{KO}-2$ & KO & hw & 1 & D-269429 & b & MOG & 49.60 & 17.20 & 11.30 & nd & 3.35 & 5.53 & 5.19 & 1.98 & 1.71 & 2.28 & 1.64 & .25 & .01 & 100.04 \\
\hline $\mathrm{KO}-3$ & KO & hw & 1 & D-269437 & $b$ & MOG & 49.40 & 16.50 & 12.10 & nd & 3.86 & 6.87 & 5.02 & 1.85 & .31 & 2.64 & 1.89 & .24 & .00 & 100.68 \\
\hline $\mathrm{KO}-4$ & KO & hw & 1 & D-269432 & $b$ & MOG & 52.70 & 17.20 & 9.57 & nd & 3.06 & 5.74 & 5.20 & 2.10 & .14 & 2.27 & 1.07 & .25 & .00 & 99.30 \\
\hline KO-5 & KO & hwt) & 1 & D-269431 & $b$ & MOG & 54.90 & 17.90 & 8.05 & nd & 2.04 & 4.15 & 5.72 & 2.36 & .96 & 1.77 & .80 & .23 & .00 & 98.88 \\
\hline $\mathrm{KO}-6$ & KO & $h w$ & 1 & D-269434 & $b$ & MOG & 49.60 & 17.00 & 11.70 & nd & 3.51 & 6.20 & 4.84 & 1.89 & 1.18 & 2.42 & 1.75 & .24 & .01 & 100.34 \\
\hline $\mathrm{KO}-7$ & $\mathrm{KO}$ & hw & 1 & D-269433 & b & MOG & 49.60 & 16.70 & 11.50 & nd & 3.63 & 6.47 & 5.01 & 1.82 & .62 & 2.48 & 1.83 & .25 & .00 & 99.91 \\
\hline $\mathrm{KO}-8$ & KO & plc & 1 & D-269438 & $b$ & MOG & 46.00 & 17.20 & 14.20 & nd & 4.68 & 7.23 & 3.63 & 1.40 & 1.36 & 3.91 & .85 & .20 & .06 & 100.72 \\
\hline $\mathrm{KO}-9$ & KO & hw & 1 & D-269448 & $b$ & MOG & 48.70 & 16.80 & 12.20 & nd & 3.29 & 6.90 & 4.75 & 1.76 & .41 & 2.63 & 1.92 & .24 & .00 & 99.60 \\
\hline KO-10 & $\mathrm{KO}$ & hw & 1 & D-283161 & b & MOG & 53.00 & 17.60 & 9.78 & nd & 2.76 & 4.83 & 5.44 & 2.16 & .65 & 1.95 & 1.34 & .27 & nd & 99.78 \\
\hline $\mathrm{KO}-11$ & $\mathrm{KO}$ & hw. & 1 & D-269435 & $b$ & MOG & 49.30 & 16.90 & 11.80 & nd & 3.66 & 6.23 & 4.73 & 1.83 & 1.21 & 2.55 & 1.87 & .25 & .00 & 100.33 \\
\hline $\mathrm{KO} \cdot 12$ & $\mathrm{KO}$ & hw & 1 & D-283167 & b & MOG & 51.50 & 17.30 & 10.90 & nd & 3.17 & 5.61 & 5.06 & 2.04 & .25 & 2.20 & 1.55 & .26 & nd & 99.84 \\
\hline $\mathrm{KO}-13$ & KO & $\mathrm{pl}$ & 1 & D-269453 & $b$ & MOG & 46.90 & 18.00 & 12.00 & nd & 4.81 & 11.30 & 3.03 & .64 & .83 & 3.00 & .48 & .16 & .00 & 101.15 \\
\hline $\mathrm{KO}-14$ & $\mathrm{KO}$ & hw & 1 & D-283164 & b & MOG & 46.50 & 16.10 & 13.80 & nd & 4.31 & 7.69 & 4.06 & 1.57 & .29 & 3.15 & 2.25 & .26 & nd & 99.98 \\
\hline $\mathrm{KO}-15$ & $\mathrm{KO}$ & hw & 1 & D-283166 & $b$ & MOG & 49.80 & 17.70 & 11.50 & nd & 2.47 & 4.85 & 4.62 & 2.04 & 2.06 & 2.35 & 1.65 & .26 & nd & 99.30 \\
\hline KO-16 & $\mathrm{KO}$ & hw & 1 & D-269436 & $b$ & MOG & 53.80 & 17.80 & 9.00 & nd & 2.41 & 5.10 & 5.14 & 2.21 & 1.26 & 2.06 & .94 & .29 & .00 & 100.01 \\
\hline KO-18 & $\mathrm{KO}$ & $\mathrm{pl}$ & 1 & D-276865 & $\mathrm{b}$ & MOG & 47.60 & 16.80 & 12.90 & nd & 4.91 & 7.69 & 4.11 & 1.51 & .00 & 3.53 & .94 & .18 & nd & 100.17 \\
\hline KO-19 & $\mathrm{KO}$ & hw & 1 & D-269439 & b & MOG & 45.00 & 16.70 & 13.80 & nd & 3.82 & 6.75 & 4.12 & 1.65 & 1.73 & 3.17 & 2.29 & .25 & .01 & 99.29 \\
\hline KO-21 & $\mathrm{KO}$ & $\mathrm{pl}$ & 1 & D-269479 & b & MOG & 47.40 & 16.00 & 13.10 & nd & 6.41 & 7.71 & 3.26 & 1.41 & .57 & 2.97 & .69 & .19 & .00 & 99.71 \\
\hline KO-22 & $\mathrm{KO}$ & hw & 1 & D-283165 & $\mathrm{b}$ & MOG & 51.40 & 18.20 & 9.65 & nd & 2.78 & 4.26 & 4.64 & 2.20 & 3.17 & 2.22 & 1.03 & .28 & nd & 99.83 \\
\hline $\mathrm{KO}-23$ & $\mathrm{KO}$ & hw & 1 & D-269442 & b & MOG & 51.50 & 18.30 & 9.11 & nd & 2.59 & 4.24 & 4.99 & 2.25 & 3.46 & 2.05 & .88 & .25 & .01 & 99.63 \\
\hline KO-24 & $\mathrm{KO}$ & pl & 1 & D-269441 & b & MOG & 48.50 & 14.40 & 14.80 & nd & 4.61 & 9.08 & 2.88 & .97 & .80 & 3.66 & .53 & .20 & .00 & 100.43 \\
\hline KO-25 & $\mathrm{KO}$ & hw & 1 & D-269440 & $b$ & MOG & 41.90 & 17.60 & 14.70 & nd & 3.83 & 5.81 & 3.23 & 1.66 & 4.55 & 3.45 & 2.50 & .25 & .02 & 99.50 \\
\hline KO-26 & $\mathrm{KO}$ & $\mathrm{pl}$ & 1 & D-269459 & b & MOG & 49.20 & 15.40 & 13.30 & nd & 5.22 & 10.50 & 2.91 & .85 & .00 & 3.22 & .42 & .18 & .00 & 101.20 \\
\hline KO-27 & $\mathrm{KO}$ & plm & 1 & D-283172 & b & MOG & 51.70 & 18.00 & 10.50 & nd & 1.95 & 5.03 & 5.42 & 2.14 & 1.43 & 2.09 & 1.47 & .25 & nd & 99.98 \\
\hline KO-28 & $\mathrm{KO}$ & $\mathrm{pl}$ & 1 & D-269469 & b & MOG & 47.00 & 14.40 & 13.50 & nd & 8.32 & 10.60 & 2.70 & .78 & .00 & 3.02 & .43 & .17 & .00 & 100.92 \\
\hline KO-29 & $\mathrm{KO}$ & hwb & 1 & D-269443 & b & MOG & 53.30 & 17.90 & 8.89 & nd & 2.22 & 4.92 & 5.23 & 2.25 & 1.25 & 2.02 & .95 & .22 & .00 & 99.15 \\
\hline $\mathrm{KO}-30$ & $\mathrm{KO}$ & $\mathrm{pl}$ & 1 & D-276875 & b & MOG & 47.90 & 14.60 & 14.70 & nd & 4.53 & 8.78 & 3.54 & 1.32 & .00 & 3.87 & .69 & .21 & nd & 100.14 \\
\hline KO-31 & $\mathrm{KO}$ & $\mathrm{pl}$ & 1 & D-269458 & b & MOG & 48.70 & 14.20 & 15.00 & nd & 5.09 & 9.58 & 2.97 & .93 & .25 & 3.66 & .50 & .21 & .00 & 101.09 \\
\hline KO-32 & KO & pim & 1 & D-276870 & b & MOG & 50.60 & 18.20 & 10.70 & nd & 2.49 & 4.85 & 5.11 & 2.10 & 1.49 & 2.08 & 1.48 & .26 & nd & 99.36 \\
\hline KO-33 & KO & $\mathrm{pl}$ & 1 & D-269461 & b & MOG & 49.30 & 15.10 & 13.60 & nd & 5.05 & 10.30 & 2.78 & .84 & .34 & 3.26 & .48 & .19 & .00 & 101.24 \\
\hline KO-34 & $\mathrm{KO}$ & hw & 1 & D-276863 & $b$ & MOG & 45.80 & 17.00 & 13.50 & nd & 3.18 & 6.41 & 4.05 & 1.76 & 2.28 & 3.03 & 2.15 & .26 & nd & 99.42 \\
\hline KO-35 & $\mathrm{KO}$ & $\mathrm{pl}$ & 1 & D-269465 & $b$ & MOG & 48.20 & 15.10 & 13.90 & nd & 4.84 & 9.23 & 3.37 & 1.24 & .15 & 3.69 & .67 & .19 & .00 & 100.58 \\
\hline KO-36 & $\mathrm{KO}$ & $\mathrm{pl}$ & 1 & D-269463 & b & MOG & 48.40 & 14.60 & 14.60 & nd & 5.07 & 10.20 & 2.93 & .90 & .00 & 3.59 & .52 & .20 & .00 & 101.01 \\
\hline KO-37 & KO & plm & 1 & D-269462 & b & MOG & 52.10 & 18.00 & 10.50 & nd & 1.89 & 4.99 & 5.31 & 2.19 & 1.24 & 2.06 & 1.49 & .20 & .00 & 99.97 \\
\hline $\mathrm{KO}-38$ & KO & $\mathrm{pl}$ & 1 & D-269485 & $\mathrm{b}$ & MOG & 46.50 & 13.90 & 15.20 & nd & 5.68 & 10.80 & 2.76 & .56 & .86 & 3.66 & .50 & .22 & .00 & 100.64 \\
\hline
\end{tabular}


Table 1.-Chemical analyses-Continued

\begin{tabular}{|c|c|c|c|c|c|c|c|c|c|c|c|c|c|c|c|c|c|c|c|c|}
\hline $\begin{array}{c}\text { Sample } \\
\text { locality } \\
\text { No. }\end{array}$ & $\begin{array}{l}\text { Vol- } \\
\text { cano }\end{array}$ & Unit & $\begin{array}{l}\text { Map } \\
\text { sheet } \\
\text { No. }\end{array}$ & $\begin{array}{c}\text { Laboratory } \\
\text { No. }\end{array}$ & Remark & Collector & $\mathrm{SiO}_{2}$ & $\mathrm{Al}_{2} \mathrm{O}_{3}$ & $\mathrm{Fe}_{2} \mathrm{O}_{3}$ & $\mathrm{FeO}$ & $\mathrm{MgO}$ & $\mathrm{CaO}$ & $\mathrm{Na}_{2} \mathrm{O}$ & $\mathrm{K}_{2} \mathrm{O}$ & $\mathrm{H}_{2} \mathrm{O}$ & $\mathrm{TiO}_{2}$ & $\mathrm{P}_{2} \mathrm{O}_{5}$ & $\mathrm{MnO}$ & $\mathrm{CO}_{2}$ & Total \\
\hline KO-39 & $\mathrm{KO}$ & $\mathrm{pl}$ & 1 & D-269466 & $\mathrm{b}$ & MOG & 48.00 & 14.30 & 15.10 & nd & 5.34 & 10.30 & 2.83 & .85 & .00 & 3.75 & .52 & .22 & .00 & 101.21 \\
\hline KO-40 & KO & $\mathrm{pl}$ & 1 & D-269487 & $b$ & MOG & 48.00 & 15.40 & 14.30 & nd & 4.64 & 8.04 & 3.60 & 1.34 & .43 & 3.96 & .77 & .20 & .00 & 100.68 \\
\hline KO-41 & KO & $\mathrm{pl}$ & 1 & D-269486 & b & MOG & 47.10 & 14.60 & 15.30 & nd & 5.18 & 10.30 & 2.83 & .73 & .33 & 3.78 & .54 & .22 & .00 & 100.91 \\
\hline KO-42 & $\mathrm{KO}$ & $\mathrm{pl}$ & 1 & D-269488 & $\mathrm{b}$ & MOG & 48.00 & 14.70 & 14.20 & nd & 5.81 & 10.20 & 2.75 & .72 & .34 & 3.47 & .50 & .21 & .00 & 100.90 \\
\hline KO-43 & KO & $\mathrm{pl}$ & 1 & D-283170 & $b$ & MOG & 48.30 & 14.70 & 13.10 & nd & 4.67 & 8.46 & 3.53 & 1.39 & 1.35 & 3.62 & .74 & .21 & nd & 100.07 \\
\hline KO-44 & $\mathrm{KO}$ & $\mathrm{pl}$ & 1 & D-276871 & b & MOG & 48.80 & 14.40 & 13.20 & nd & 5.61 & 10.50 & 2.90 & .91 & .00 & 3.45 & .51 & .19 & nd & 100.47 \\
\hline KO-45 & $\mathrm{KO}$ & hwb & 1 & D-276864 & b & MOG & 54.00 & 17.20 & 8.91 & nd & 2.80 & 5.44 & 5.63 & 2.21 & .10 & 2.02 & .94 & .24 & nd & 99.49 \\
\hline KO-46 & $\mathrm{KO}$ & pl & 1 & D-269472 & b & MOG & 48.50 & 15.50 & 12.50 & nd & 6.22 & 10.90 & 2.68 & .79 & .05 & 2.93 & .42 & .17 & .00 & 100.66 \\
\hline $\mathrm{KO}-47$ & KO & pl & 1 & D-283169 & $b$ & MOG & 47.30 & 16.80 & 13.50 & nd & 5.04 & 7.62 & 3.72 & 1.43 & .19 & 3.69 & .86 & .19 & nd & 100.34 \\
\hline $\mathrm{KO}-48$ & KO & $\mathrm{pl}$ & 1 & D-276872 & b & MOG & 47.40 & 14.80 & 13.90 & nd & 5.02 & 7.87 & 3.63 & 1.37 & .61 & 3.77 & .77 & .20 & nd & 99.34 \\
\hline KO-49 & KO & $\mathrm{pl}$ & 1 & D-269484 & b & MOG & 47.30 & 16.70 & 13.50 & nd & 4.94 & 7.66 & 3.81 & 1.45 & .41 & 3.68 & .85 & .19 & .00 & 100.49 \\
\hline KO-50 & $\mathrm{KO}$ & plc & 1 & D-269482 & $b$ & MOG & 48.70 & 14.40 & 14.50 & nd & 5.51 & 10.50 & 2.81 & .52 & .11 & 3.35 & .41 & .21 & .00 & 101.02 \\
\hline KO-51 & $\mathrm{KO}$ & $\mathrm{pl}$ & 1 & D-276873 & b & MOG & 47.80 & 13.90 & 12.70 & nd & 6.20 & 10.60 & 2.65 & .87 & 1.51 & 3.12 & .49 & .15 & nd & 99.99 \\
\hline KO-52 & $\mathrm{KO}$ & $\mathrm{pl}$ & 1 & D-269445 & b & MOG & 47.70 & 14.90 & 14.10 & nd & 5.10 & 7.99 & 3.59 & 1.34 & 1.01 & 3.83 & .77 & .22 & .02 & 100.57 \\
\hline KO-53 & $\mathrm{KO}$ & $\mathrm{pl}$ & 1 & D-276868 & $\mathrm{b}$ & MOG & 46.80 & 16.50 & 13.60 & nd & 5.08 & 7.67 & 3.80 & 1.42 & .13 & 3.72 & .85 & .19 & nd & 99.76 \\
\hline KO-54 & $\mathrm{KO}$ & plc & 1 & D-269473 & $b$ & MOG & 49.90 & 15.00 & 13.20 & nd & 5.30 & 10.50 & 2.72 & .70 & .00 & 3.00 & .38 & .18 & .01 & 100.89 \\
\hline KO-55 & $\mathrm{KO}$ & $\mathrm{pl}$ & 1 & D-276869 & b & MOG & 47.90 & 13.80 & 13.20 & nd & 6.98 & 9.97 & 2.71 & .85 & 1.24 & 3.06 & .49 & .19 & nd & 100.39 \\
\hline KO-56 & KO & pl & 1 & D-276866 & b & MOG & 48.40 & 14.20 & 14.70 & nd & 4.23 & 8.10 & 3.75 & 1.33 & .00 & 4.02 & .81 & .21 & nd & 99.75 \\
\hline KO-57 & $\mathrm{KO}$ & pl & 1 & D-276867 & b & MOG & 48.60 & 13.10 & 13.00 & nd & 8.18 & 10.80 & 2.39 & .70 & .00 & 2.91 & .39 & .18 & nd & 100.25 \\
\hline KP-1 & $\mathrm{KO}$ & $\mathrm{pl}$ & 1 & D-277468 & $b$ & $\mathrm{SCP}$ & 50.00 & 13.40 & 12.10 & nd & 9.70 & 10.90 & 1.97 & .18 & .45 & 2.14 & .21 & .16 & nd & 101.21 \\
\hline $\mathrm{KP}-2$ & $\mathrm{KO}$ & hw & 1 & D-277485 & $b$ & SCP & 53.20 & 16.80 & 9.71 & nd & 2.90 & 5.37 & 5.74 & 2.55 & .00 & 1.99 & 1.33 & .21 & nd & 99.80 \\
\hline $\mathrm{KP}-3$ & $\mathrm{KO}$ & hw & 1 & D-277484 & b & SCP & 52.40 & 17.30 & 10.20 & nd & 2.94 & 5.56 & 5.46 & 2.27 & .25 & 2.06 & 1.40 & .24 & nd & 100.08 \\
\hline $\mathrm{KP}-4$ & KO & $\mathrm{pl}$ & 1 & D-277470 & $b$ & SCP & 48.00 & 11.00 & 12.60 & nd & 15.60 & 9.21 & 1.68 & .18 & .04 & 1.78 & .17 & .17 & nd & 100.43 \\
\hline $\mathrm{KP}-5$ & KO & $\mathrm{pl}$ & 1 & D-277469 & $b$ & SCP & 50.80 & 13.40 & 12.40 & nd & 7.83 & 11.10 & 2.28 & .35 & .00 & 2.43 & .24 & .17 & nd & 101.00 \\
\hline $\mathrm{KP}-6$ & KO & pl & 1 & D-277471 & $b$ & SCP & 49.40 & 14.30 & 12.60 & nd & 7.27 & 11.60 & 2.31 & .15 & .35 & 2.45 & .24 & .18 & nd & 100.85 \\
\hline KP-7 & KO & hw & 1 & D-269137 & b & SCP & 53.00 & 17.30 & 9.08 & nd & 2.81 & 5.08 & 5.33 & 2.19 & 1.34 & 2.09 & .99 & .26 & .00 & 99.47 \\
\hline $\mathrm{KP}-8$ & $\mathrm{KO}$ & $\mathrm{pl}$ & 1 & D-269136 & b & SCP & 47.10 & 14.40 & 14.00 & nd & 4.16 & 9.62 & 2.89 & .92 & 1.88 & 4.15 & .56 & .16 & .00 & 99.84 \\
\hline KP-9 & $\mathrm{KO}$ & hw & 1 & D-277467 & b & SCP & 52.60 & 17.50 & 9.20 & nd & 2.87 & 4.90 & 5.27 & 2.22 & 1.78 & 2.13 & 1.02 & .26 & nd & 99.75 \\
\hline KP-11 & KO & $\mathrm{pl}$ & 1 & D-269134 & b & SCP & 48.90 & 14.10 & 13.40 & nd & 6.12 & 10.40 & 2.85 & .74 & .00 & 2.94 & .40 & .19 & .00 & 100.04 \\
\hline $\mathrm{Ki}-1$ & MK & $\mathrm{hm}$ & 2 & $\mathrm{Ki}-1$ & $d$ & WSW & 45.73 & 13.06 & 13.79 & nd & 9.82 & 10.82 & 2.23 & .64 & nd & 3.09 & .36 & .18 & nd & 99.72 \\
\hline $\mathrm{Ki}-2$ & MK & $\mathrm{hm}$ & 2 & $\mathrm{Ki}-2$ & d & WSW & 45.48 & 12.78 & 13.90 & nd & 10.20 & 10.77 & 2.42 & .64 & nd & 3.11 & .35 & .18 & nd & 99.83 \\
\hline $\mathrm{Ki}-3$ & MK & $\mathrm{hm}$ & 2 & $\mathrm{Ki}-3$ & d & WSW & 44.38 & 7.63 & 13.30 & nd & 24.24 & 7.38 & 1.08 & .08 & nd & 1.55 & .16 & .19 & nd & 99.99 \\
\hline $\mathrm{Ki}-4$ & MK & $\mathrm{hm}$ & 2 & $\mathrm{Ki}-4$ & d & WSW & 45.99 & 9.99 & 13.40 & nd & 17.22 & 9.47 & 1.40 & .21 & nd & 2.17 & .21 & .17 & nd & 100.23 \\
\hline $\mathrm{Ki}-5$ & MK & $\mathrm{hm}$ & 2 & $\mathrm{Ki}-5$ & $d$ & WSW & 46.78 & 9.76 & 12.90 & nd & 16.91 & 9.39 & 1.25 & .39 & nd & 2.09 & .21 & .19 & nd & 99.87 \\
\hline $\mathrm{Ki}-6$ & MK & $\mathrm{hm}$ & 2 & $\mathrm{Ki}-6$ & d & WSW & 45.65 & 13.10 & 13.89 & nd & 9.14 & 10.84 & 2.45 & .70 & nd & 3.28 & .39 & .18 & nd & 99.62 \\
\hline $\mathrm{Ki}-7$ & MK & $\mathrm{hm}$ & 2 & $\mathrm{Ki}-7$ & $d$ & WSW & 45.71 & 13.41 & 13.93 & nd & 8.68 & 11.08 & 2.17 & .66 & nd & 3.41 & .38 & .19 & nd & 99.62 \\
\hline $\mathrm{Ki}-8$ & MK & $\mathrm{hm}$ & 2 & $\mathrm{Ki}-8$ & d & WSW & 46.25 & 8.78 & 12.71 & nd & 17.89 & 10.29 & 1.76 & .12 & nd & 1.81 & .19 & .16 & nd & 99.96 \\
\hline $\mathrm{Ki}-9$ & MK & $\mathrm{hm}$ & 2 & Ki-9 & d & WSW & 47.22 & 13.57 & 13.76 & nd & 8.60 & 11.23 & 2.27 & .17 & nd & 2.76 & .29 & .19 & nd & 100.06 \\
\hline $\mathrm{Ki}-10$ & MK & $\mathrm{hm}$ & 2 & $\mathrm{Ki}-10$ & $\mathrm{~d}$ & WSW & 48.19 & 14.14 & 13.24 & nd & 6.38 & 11.25 & 2.55 & .70 & nd & 3.03 & .35 & .18 & nd & 100.01 \\
\hline $\mathrm{Ki}-11$ & MK & $\mathrm{hm}$ & 2 & $\mathrm{Ki}-11$ & $d$ & WSW & 46.23 & 7.61 & 12.31 & nd & 20.13 & 10.66 & .95 & .23 & nd & 1.46 & .15 & .16 & nd & 99.89 \\
\hline
\end{tabular}


Table 1.-Chemical analyses-Continued

\begin{tabular}{|c|c|c|c|c|c|c|c|c|c|c|c|c|c|c|c|c|c|c|c|c|}
\hline $\begin{array}{c}\text { Sample } \\
\text { locality } \\
\text { No. }\end{array}$ & $\begin{array}{l}\text { Vol- } \\
\text { cano }\end{array}$ & Unit & $\begin{array}{c}\text { Map } \\
\text { sheet } \\
\text { No. }\end{array}$ & $\begin{array}{c}\text { Laboratory } \\
\text { No. }\end{array}$ & Remark & Collector & $\mathrm{SiO}_{2}$ & $\mathrm{Al}_{2} \mathrm{O}_{3}$ & $\mathrm{Fe}_{2} \mathrm{O}_{3}$ & $\mathrm{FeO}$ & $\mathrm{MgO}$ & $\mathrm{CaO}$ & $\mathrm{Na}_{2} \mathrm{O}$ & $\mathrm{K}_{2} \mathrm{O}$ & $\mathrm{H}_{2} \mathrm{O}$ & $\mathrm{TiO}_{2}$ & $\mathrm{P}_{2} \mathrm{O}_{5}$ & $\mathrm{MnO}$ & $\mathrm{CO}_{2}$ & Total \\
\hline $\mathrm{Ki}-101$ & $\mathrm{MK}$ & $\mathrm{hm}$ & 2 & 13730 & $e$ & KJM & 47.07 & 9.73 & 2.52 & 9.31 & 18.68 & 8.48 & 1.59 & .32 & .21 & 1.90 & .20 & .18 & .01 & 100.20 \\
\hline La1 & MK & $\mathrm{hm}$ & 2 & La-1 & d & WSW & 45.97 & 13.82 & 14.00 & nd & 8.49 & 10.35 & 2.74 & .55 & .70 & 3.26 & .49 & .20 & nd & 100.57 \\
\hline La2 & MK & $\mathrm{hm}$ & 2 & La-2 & d & WSW & 47.72 & 14.60 & 14.20 & nd & 6.62 & 10.84 & 2.44 & .19 & .82 & 3.17 & .34 & .19 & nd & 101.13 \\
\hline La3 & MK & $\mathrm{hm}$ & 2 & La-3 & d & WSW & 49.37 & 13.24 & 13.20 & nd & 6.61 & 10.68 & 2.32 & .54 & .38 & 2.93 & .30 & .17 & nd & 99.74 \\
\hline $\operatorname{La}-4$ & MK & $\mathrm{hm}$ & 2 & $\mathrm{La}-4$ & d & WSW & 47.16 & 10.32 & 12.89 & nd & 14.20 & 10.79 & 1.84 & .45 & .11 & 2.31 & .25 & .18 & nd & 100.50 \\
\hline La 5 & MK & $\mathrm{hm}$ & 2 & La-5 & d & WSW & 46.72 & 10.66 & 14.40 & nd & 12.57 & 9.62 & 2.20 & .71 & .30 & 2.83 & .34 & .20 & nd & 100.55 \\
\hline La 6 & MK & $\mathrm{hm}$ & 2 & La 6 & d & WSW & 44.43 & 10.47 & 14.34 & nd & 15.90 & 8.82 & 1.74 & .32 & .43 & 2.53 & .31 & .19 & nd & 99.48 \\
\hline $\operatorname{La} 7$ & MK & $\mathrm{hm}$ & 2 & La-7 & $d$ & WSW & 47.62 & 13.34 & 13.56 & nd & 7.77 & 9.73 & 3.13 & 1.05 & .81 & 3.17 & .50 & .19 & nd & 100.87 \\
\hline La8 8 & MK & $\mathrm{hm}$ & 2 & La-8 & d & WSW & 46.58 & 13.20 & 13.80 & nd & 8.55 & 9.92 & 3.17 & .92 & .32 & 3.17 & .48 & .19 & nd & 100.30 \\
\hline La9 & MK & $\mathrm{hm}$ & 2 & La-9 & d & WSW & 45.56 & 12.97 & 14.00 & nd & 9.66 & 10.74 & 2.85 & .72 & .26 & 3.20 & .38 & .19 & nd & 100.53 \\
\hline La-10 & MK & $\mathrm{hm}$ & 2 & La-10 & $d$ & WSW & 45.49 & 13.03 & 14.00 & nd & 9.64 & 10.74 & 2.44 & .69 & .44 & 3.19 & .38 & .19 & nd & 100.23 \\
\hline La-11 & MK & $\mathrm{hm}$ & 2 & La-11 & d & WSW & 49.46 & 13.43 & 13.58 & nd & 6.05 & 10.30 & 2.51 & .60 & .14 & 3.02 & .32 & .19 & nd & 99.60 \\
\hline La-101 & MK & $\mathrm{hm}$ & 2 & 14174 & e & $\mathrm{KJM}$ & 45.50 & 13.56 & 5.22 & 7.85 & 9.88 & 9.66 & 2.33 & .47 & 1.81 & 3.20 & .39 & .18 & .01 & 100.06 \\
\hline La-102 & $\mathrm{MK}$ & $\mathrm{hm}$ & 2 & $\mathrm{I} 3726$ & $e$ & KJM & 47.28 & 13.98 & 3.27 & 9.00 & 8.07 & 11.25 & 2.40 & .43 & .70 & 3.03 & .34 & .18 & .00 & 99.93 \\
\hline La-103 & MK & $\mathrm{hm}$ & 2 & 13727 & $e$ & $\mathrm{KJM}$ & 45.32 & 14.86 & 3.90 & 11.25 & 4.96 & 7.89 & 3.41 & 1.65 & .75 & 4.79 & .88 & .22 & .01 & 99.89 \\
\hline La-104 & MK & $\mathrm{hm}$ & 2 & I3728 & $e$ & KJM & 47.93 & 14.44 & 3.16 & 10.35 & 5.79 & 10.58 & 2.89 & .84 & .11 & 3.37 & .42 & .20 & .01 & 100.09 \\
\hline La-105 & MK & $\mathrm{hm}$ & 2 & K3580 & e & $\mathrm{KJM}$ & 44.70 & 8.42 & 3.40 & 8.86 & 22.03 & 8.22 & 1.17 & .14 & .86 & 1.79 & .18 & .18 & .04 & 99.99 \\
\hline Mut2 & $\mathrm{MK}$ & $\mathrm{hm}$ & 2 & Mu-2 & d & WSW & 47.68 & 14.98 & 13.25 & nd & 6.82 & 11.91 & 2.14 & .20 & .29 & 2.62 & .27 & .19 & nd & 100.35 \\
\hline Mu-3 & $\mathrm{MK}$ & $\mathrm{hm}$ & 2 & Mu-3 & d & WSW & 47.83 & 14.35 & 14.52 & nd & 5.93 & 10.79 & 2.52 & .31 & .31 & 3.32 & .35 & .19 & nd & 100.42 \\
\hline $\mathrm{Mu}-4$ & MK & $\mathrm{hm}$ & 2 & $\mathrm{Mu}-4$ & $d$ & Wsw & 46.65 & 13.96 & 13.73 & nd & 8.05 & 11.51 & 2.04 & .19 & .49 & 3.03 & .31 & .17 & nd & 100.13 \\
\hline Mu-5 & MK & $\mathrm{hm}$ & 2 & $\mathrm{Mu}-5$ & d & WSW & 45.93 & 14.32 & 13.49 & nd & 7.93 & 10.18 & 2.80 & .88 & .09 & 3.28 & .41 & .17 & nd & 99.48 \\
\hline Mut6 & MK & $\mathrm{hm}$ & 2 & $\mathrm{Mu}-6$ & d & WSW & 44.28 & 15.24 & 16.22 & nd & 5.76 & 9.29 & 2.84 & .67 & .80 & 4.73 & .70 & .20 & nd & 100.73 \\
\hline Mu-7 & MK & $\mathrm{hm}$ & 2 & $\mathrm{Mu}-7$ & $d$ & WSW & 47.47 & 14.28 & 14.55 & nd & 6.28 & 11.13 & 2.36 & .40 & .38 & 3.25 & .34 & .19 & nd & 100.63 \\
\hline $\mathrm{Mu}-8$ & MK & $\mathrm{hm}$ & 2 & $\mathrm{Mu}-8$ & $\mathrm{~d}$ & WSW & 47.02 & 12.19 & 13.76 & nd & 10.76 & 11.24 & 1.39 & .18 & .71 & 2.74 & .29 & .19 & nd & 100.47 \\
\hline Mu-9 & MK & $\mathrm{hm}$ & 2 & $\mathrm{Mu}-9$ & d & WSW & 45.93 & 9.62 & 13.53 & nd & 15.93 & 10.80 & 1.26 & .22 & .53 & 2.04 & .22 & .19 & nd & 100.27 \\
\hline $\mathrm{Mu}-10$ & MK & $\mathrm{hm}$ & 2 & $\mathrm{Mu}-10$ & d & WSW & 45.48 & 11.58 & 14.01 & nd & 14.28 & 9.75 & 1.71 & .21 & .43 & 2.53 & .29 & .18 & nd & 100.45 \\
\hline $\mathrm{Mu}-11$ & MK & $\mathrm{hm}$ & 2 & $\mathrm{Mu}-11$ & d & WSW & 46.18 & 14.61 & 15.74 & nd & 5.15 & 8.57 & 2.90 & 1.20 & 1.56 & 4.83 & .61 & .21 & nd & 101.56 \\
\hline $\mathrm{Mu}-13$ & MK & $\mathrm{hm}$ & 2 & $\mathrm{Mu}-13$ & d & WSW & 47.12 & 14.82 & 14.41 & nd & 6.03 & 10.62 & 2.66 & .22 & .85 & 3.29 & .33 & .19 & nd & 100.54 \\
\hline $\mathrm{Mu}-14$ & MK & $\mathrm{hm}$ & 2 & $\mathrm{Mu}-14$ & d & WSW & 46.85 & 15.01 & 14.96 & nd & 6.25 & 10.01 & 2.61 & .21 & 1.30 & 3.42 & .35 & .20 & nd & 101.17 \\
\hline Mu-15 & MK & $\mathrm{hm}$ & 2 & $\mathrm{Mu}-15$ & $d$ & WSW & 46.57 & 14.48 & 14.46 & nd & 6.74 & 11.42 & 2.18 & .18 & .54 & 3.32 & .32 & .20 & nd & 100.41 \\
\hline PuuAna & $\mathrm{HU}$ & $w$ & 1 & & $f$ & & 62.02 & 18.71 & 4.30 & .10 & .40 & .86 & 6.90 & 4.93 & 1.11 & .31 & .24 & .15 & .00 & 100.03 \\
\hline PuuWaa & $\mathrm{HU}$ & wc & 1 & & $g$ & & 62.19 & 17.43 & 1.65 & 2.64 & .40 & .86 & 8.28 & 5.03 & .53 & .37 & .14 & .32 & .02 & 99.86 \\
\hline
\end{tabular}


lable 2.--Radiocarbon ages

[Radiocarbon ages determined mostly from charcoal collected beneath dated lava flows, spatter deposits, or tephra deposits. Quadrangle code: see figure 1. Volcano: HU, Hualalai; KI, Kilauea; MK, Mauna Kea; ML, Mauna Loa. Map. unit designations correspond to geologic map units shown on Map A. Sample collectors: JPL, J.P. Lockwood; PWL, P.W. Lipman; KJM, K.J. Murata; RRD, R.R. Doell; NGB, N.G. Banks; MDJ, M.D. Jackson; RTH, R.T. Holcomb; CAN, C.A. Neal; RIT, R.I. Tilling; RME, R.M. Easton; FAT, F.A. Trusdell; RBM, R.B. Moore; DMD, D. Mueller-Dombois; CDC, C.D. Condit; DAC, D.A. Clague; RID, R.I. Dorn; EWW, E.W. Wolfe; GAM, G.A. Macdonald; JBB, J.M. Buchanan-Banks; WSW, W.S. Wise; MAK, M.A. Kuntz; PMV, P.M. Vitousek.

Remarks:

a Charcoal ages reported by Rubin and others (1987).

b Charcoal ages determined by U.S. Geological Survey Radiocarbon Laboratory, Reston, Va. (M. Rubin, written commun., 1987-1991)

c Charcoal ages determined by accelerator mass spectrometry, University of Arizona (M. Rubin, written commun.,, 1988-1993).

d Varnish radiocarbon age (Dorn and others, 1992).

e Charcoal age reported by Mueller-Dombois and Lamoreux (1967).]

\begin{tabular}{|c|c|c|c|c|c|c|c|}
\hline $\begin{array}{l}\text { Quadrangle } \\
\text { code }\end{array}$ & Volcano & $\begin{array}{c}\text { Dated map } \\
\text { unit }\end{array}$ & $\begin{array}{l}\text { Map sheet } \\
\text { No. }\end{array}$ & $\begin{array}{c}\text { Laboratory } \\
\text { No. }\end{array}$ & Remark & Collector & $\begin{array}{c}{ }^{14} \mathrm{C} \text { age (years } \\
\text { B.P.) }\end{array}$ \\
\hline AP & ML & $\mathrm{k}$ & 3 & W 3487 & a & JPL & $11,780 \pm 100$ \\
\hline AP & ML & $\mathrm{k} 10$ & 3 & W4351 & a & PWL & $7,750 \pm 70$ \\
\hline BP & ML & $\mathrm{kc} 4$ & 3 & W 4238 & a & PWL & $300 \pm 70$ \\
\hline $\mathrm{BQ}$ & ML & $\mathrm{k} 1 \mathrm{y}$ & 3 & W856 & a & KJM & $3,740 \pm 250$ \\
\hline$B Q$ & ML & $\mathrm{k} 1 \mathrm{y}$ & 3 & W2016 & a & RRD & $3,620 \pm 250$ \\
\hline $\mathrm{BQ}$ & ML & $\mathrm{k} 1 \mathrm{o}$ & 3 & W 3930 & a & PWL & $6,160 \pm 110$ \\
\hline$B Q$ & ML & $\mathrm{k}$ & 3 & W3935 & a & PWL & $31,100 \pm 900$ \\
\hline$B Q$ & ML & $\mathrm{k} 2$ & 3 & W4015 & a & PWL & $2,180 \pm 60$ \\
\hline $\mathrm{BQ}$ & ML & $\mathrm{k}$ & 3 & W4 160 & a & PWL & $10,290 \pm 150$ \\
\hline$B Q$ & ML & $\mathrm{k} 2$ & 3 & W 4377 & a & PWL & $2,650 \pm 50$ \\
\hline$C P$ & ML & $\mathrm{k} 4$ & 3 & W4025 & a & PWL & $640 \pm 45$ \\
\hline $\mathrm{CQ}$ & ML & $\mathrm{k} 1 \mathrm{o}$ & 3 & W 3813 & a & PWL & $7,950 \pm 110$ \\
\hline $\mathrm{CQ}$ & ML & $\mathrm{k} 2$ & 3 & W4008 & $\mathbf{a}$ & PWL & $2,300 \pm 60$ \\
\hline $\mathrm{CQ}$ & ML & $k 1 y$ & 3 & W4009 & a & PWL & $3,900 \pm 80$ \\
\hline $\mathrm{CQ}$ & ML & k3 & 3 & W4012 & a & PWL & $740 \pm 60$ \\
\hline $\mathrm{CQ}$ & ML & $\mathrm{k} 2$ & 3 & W4022 & a & PWL & $1,860 \pm 70$ \\
\hline $\mathrm{CQ}$ & ML & $\mathrm{k} 1 \mathrm{y}$ & 3 & W4 132 & a & PWL & $3,900 \pm 90$ \\
\hline $\mathrm{CQ}$ & ML & k3 & 3 & W4 137 & a & PWL & $890 \pm 60$ \\
\hline $\mathrm{CQ}$ & ML & $\mathrm{k} 2$ & 3 & W4142 & a & PWL & $2,440 \pm 60$ \\
\hline $\mathrm{CQ}$ & ML & $\mathrm{k} 1 \mathrm{y}$ & 3 & W4 152 & a & PWL & $3,800 \pm 90$ \\
\hline $\mathrm{CQ}$ & ML & $\mathrm{k} 3$ & 3 & W4 156 & $\mathbf{a}$ & PWL & $740 \pm 60$ \\
\hline $\mathrm{CQ}$ & ML & $\mathrm{k} 1 \mathrm{o}$ & 3 & W4 201 & a & PWL & $9,170 \pm 100$ \\
\hline $\mathrm{CQ}$ & ML & $\mathrm{k} 1 \mathrm{y}$ & 3 & W4223 & $\mathbf{a}$ & PWL & $3,600 \pm 70$ \\
\hline $\mathrm{CQ}$ & ML & $\mathrm{k} 1 \mathrm{y}$ & 3 & W 4224 & a & PWL & $4,770 \pm 90$ \\
\hline $\mathrm{CQ}$ & ML & k3 & 3 & W4231 & a & PWL & $910 \pm 60$ \\
\hline $\mathrm{CR}$ & ML & k3 & 3 & W 3857 & a & PWL & $1,470 \pm 60$ \\
\hline $\mathrm{CR}$ & ML & k3 & 3 & W3858 & a & PWL & $1,400 \pm 60$ \\
\hline $\mathrm{CR}$ & $\mathrm{KI}$ & $\mathrm{p} 40$ & 3 & W 3860 & a & PWL & $670 \pm 60$ \\
\hline CR & $\mathrm{KI}$ & $\mathrm{p} 1 \mathrm{y}$ & 3 & W 3884 & a & PWL & $3,610 \pm 60$ \\
\hline $\mathrm{CR}$ & $\mathrm{KI}$ & $\mathrm{p} 40$ & 3 & W4404 & a & NGB & $530 \pm 70$ \\
\hline $\mathrm{CR}$ & $\mathrm{KI}$ & $\mathrm{p} 40$ & 3 & W4410 & a & NGB & $660 \pm 70$ \\
\hline $\mathrm{CR}$ & ML & k3 & 3 & W4414 & a & NGB & $1,330 \pm 60$ \\
\hline $\mathrm{CR}$ & ML & $\mathrm{k} 10$ & 3 & W44 19 & a & NGB & $9,300 \pm 130$ \\
\hline CR & ML & k3 & 3 & W5 147 & a & NGB & $890 \pm 70$ \\
\hline CR & $\mathrm{KI}$ & $\mathrm{p} 40$ & 3 & W5218 & a & NGB & $550 \pm 70$ \\
\hline CR & $\mathrm{KI}$ & $\mathrm{p} 1 \mathrm{y}$ & 3 & W5324 & a & NGB & $3,500 \pm 200$ \\
\hline CR & $\mathrm{ML}$ & k3 & 3 & W5325 & a & NGB & $1,370 \pm 200$ \\
\hline CR & ML & $\mathrm{k} 3$ & 3 & W6037 & $b$ & MDJ & $1,290 \pm 120$ \\
\hline $\mathrm{CS}$ & $\mathrm{KI}$ & $\mathrm{p} 2$ & 2 & W4119 & $\mathrm{a}$ & RTH & $1,800 \pm 80$ \\
\hline $\mathrm{CS}$ & $\mathrm{KI}$ & p 40 & 2 & W5932 & $b$ & CAN & $462 \pm 111$ \\
\hline $\mathrm{DQ}$ & $\mathrm{ML}$ & $\mathrm{k} 2$ & 3 & W4017 & a & PWL & $2,010 \pm 70$ \\
\hline $\mathrm{DQ}$ & ML & $\mathrm{k} 2$ & 3 & W 4161 & $\mathrm{a}$ & PWL & $2,000 \pm 70$ \\
\hline $\mathrm{DQ}$ & ML & $\mathrm{k} 2$ & 3 & W4 163 & a & PWL & $1,980 \pm 80$ \\
\hline $\mathrm{DQ}$ & $\mathrm{ML}$ & $\mathrm{k} 1 \mathrm{y}$ & 3 & W4 164 & a & PWL & $3,750 \pm 70$ \\
\hline
\end{tabular}


Table 2.-Radiocarbon ages-Continued

\begin{tabular}{|c|c|c|c|c|c|c|c|}
\hline $\begin{array}{l}\text { Quadrangle } \\
\text { code }\end{array}$ & Volcano & $\begin{array}{l}\text { Dated map } \\
\text { unit }\end{array}$ & $\begin{array}{c}\text { Map sheet } \\
\text { No. }\end{array}$ & $\begin{array}{l}\text { Laboratory } \\
\text { No. }\end{array}$ & Remark & Collector & $\begin{array}{c}{ }^{14} \mathrm{C} \text { age (years } \\
\text { B.P.) }\end{array}$ \\
\hline DR & $\mathrm{ML}$ & $\mathrm{k} 3$ & 3 & W3000 & $\bar{a}$ & RIT & $1,330 \pm 70$ \\
\hline DR & ML & $\mathrm{k} 2$ & 3 & W3841 & $\mathrm{a}$ & PWL & $2,950 \pm 80$ \\
\hline DR & $\mathrm{KI}$ & $\mathrm{p} 4 \mathrm{o}$ & 3 & W 3842 & $\mathrm{a}$ & PWL & $450 \pm 60$ \\
\hline DR & ML & $\mathrm{k} 2$ & 3 & W 3845 & a & PWL & $2,880 \pm 70$ \\
\hline DR & $\mathrm{KI}$ & $\mathrm{p} 4 \mathrm{y}$ & 3 & W3938 & a & $\mathrm{RTH}$ & $210 \pm 60$ \\
\hline DR & ML & k3 & 3 & W 4430 & $\mathrm{a}$ & NGB & $1,270 \pm 70$ \\
\hline DR & ML & $\mathrm{k} 10$ & 3 & W 4821 & $\mathrm{a}$ & JPL & $8,550 \pm 90$ \\
\hline DR & ML & k3 & 3 & W5458 & $\mathrm{a}$ & JPL & $1,640 \pm 150$ \\
\hline DR & ML & k3 & 3 & W5461 & $\mathrm{a}$ & JPL & $1,510 \pm 110$ \\
\hline DR & ML & $\mathrm{k} 3$ & 3 & W5679 & $\mathrm{a}$ & MDJ & $1,360 \pm 150$ \\
\hline DR & $\mathrm{KI}$ & $\mathrm{p} 40$ & 3 & W5 757 & $b$ & CAN & $410 \pm 150$ \\
\hline DR & $\mathrm{KI}$ & $\mathrm{p} 4 \mathrm{o}$ & 3 & W5771 & $b$ & CAN & $590 \pm 100$ \\
\hline DR & ML & k3 & 3 & W5982 & $b$ & MDJ & $1,460 \pm 100$ \\
\hline DR & ML & $\mathrm{k} 3$ & 3 & W6012 & $b$ & MDJ & $1,360 \pm 160$ \\
\hline DR & ML & $\mathrm{k} 1 \mathrm{o}$ & 3 & W6038 & $\mathrm{b}$ & MDJ & $8,370 \pm 200$ \\
\hline DS & $\mathrm{KI}$ & pha & 2 & W 3801 & a & RME & $22,500 \pm 300$ \\
\hline DS & $\mathrm{KI}$ & pha & 2 & W 3814 & $\mathrm{a}$ & RME & $22,800 \pm 340$ \\
\hline DS & $\mathrm{KI}$ & $\mathrm{p} 40$ & 2 & W4402 & a & NGB & $700 \pm 70$ \\
\hline DS & $\mathrm{KI}$ & p3 & 2 & W5 5135 & a & NGB & $1,150 \pm 70$ \\
\hline DS & $\mathrm{KI}$ & $\mathrm{p} 40$ & 2 & W5 145 & $\mathrm{a}$ & NGB & $500 \pm 100$ \\
\hline DS & $\mathrm{KI}$ & $p 40$ & 2 & W5152 & a & NGB & $660 \pm 70$ \\
\hline DS & $\mathrm{KI}$ & p3 & 2 & W5212 & $\mathrm{a}$ & NGB & $1,140 \pm 70$ \\
\hline DS & $\mathrm{KI}$ & $\mathrm{p} 40$ & 2 & W5 5785 & $\mathrm{~b}$ & $\mathrm{CAN}$ & $600 \pm 100$ \\
\hline DS & $\mathrm{KI}$ & $\mathrm{p} 40$ & 2 & W5973 & $\mathrm{b}$ & $\mathrm{CAN}$ & $740 \pm 100$ \\
\hline DS & $\mathrm{KI}$ & p 3 & 2 & W5975 & $\mathrm{b}$ & CAN & $900 \pm 120$ \\
\hline DT & $\mathrm{KI}$ & p4 & 2 & W 4337 & $\mathrm{a}$ & JPL & $620 \pm 70$ \\
\hline DT & $\mathrm{KI}$ & p4 & 2 & W5 110 & $\mathrm{a}$ & JPL & $230 \pm 60$ \\
\hline DT & $\mathrm{KI}$ & $\mathrm{p} 4 \mathrm{o}$ & 2 & W5983 & $b$ & RTH & $550 \pm 100$ \\
\hline DU & $\mathrm{KI}$ & $\mathrm{p} 2$ & 2 & W5052 & $\mathrm{a}$ & $\mathrm{RBM}$ & $2,360 \pm 90$ \\
\hline $\mathrm{DU}$ & $\mathrm{KI}$ & $p 4 y$ & 2 & W6269 & $\mathrm{b}$ & FAT & $350 \pm 90$ \\
\hline EN & ML & $\mathrm{k}$ & 3 & W4 128 & a & PWL & $31,400 \pm 2,000$ \\
\hline EN & ML & $\mathrm{k} 2$ & 3 & W6058 & b & JPL & $2,940 \pm 200$ \\
\hline EO & ML & $\mathrm{k} 4$ & 3 & W5627 & $\mathrm{b}$ & JPL & $750 \pm 200$ \\
\hline EO & ML & $\mathrm{k} 2$ & 3 & W5630 & $b$ & JPL & $2,050 \pm 150$ \\
\hline EO & ML & k3 & 3 & W6049 & $b$ & JPL & $1,170 \pm 100$ \\
\hline EO & ML & $\mathrm{k} 2$ & 3 & W6056 & $\mathrm{b}$ & JPL & $2,120 \pm 160$ \\
\hline EO & ML & $\mathrm{k} 1 \mathrm{y}$ & 3 & W6064 & $\mathrm{b}$ & JPL & $3,270 \pm 180$ \\
\hline EP & ML & $\mathrm{k} 3$ & 3 & W5537 & a & JPL & $1,120 \pm 200$ \\
\hline EQ & $\mathrm{ML}$ & $\mathrm{k} 3$ & 3 & W3910 & $\mathrm{a}$ & JPL & $1,270 \pm 60$ \\
\hline EQ & ML & k3 & 3 & W5083 & a & JPL & $1,030 \pm 60$ \\
\hline ER & ML & k3 & 3 & W 4047 & a & JPL & $910 \pm 70$ \\
\hline ER & ML & $\mathrm{k} 2$ & 3 & W4116 & $\mathrm{a}$ & JPL & $1,840 \pm 60$ \\
\hline ER & ML & $\mathrm{k} 4$ & 3 & W4 4183 & $\mathrm{a}$ & JPL & $300 \pm 60$ \\
\hline ER & ML & $\mathrm{k} 4$ & 3 & W 4790 & $\mathrm{a}$ & JPL & $540 \pm 70$ \\
\hline ER & ML & k3 & 3 & W4863 & a & JPL & $780 \pm 70$ \\
\hline ER & ML & $\mathrm{k} 4$ & 3 & W6001 & b & JPL & $720 \pm 100$ \\
\hline ER & ML & $\mathrm{k} 2$ & 3 & W6004 & $\mathrm{b}$ & JPL & $1,910 \pm 120$ \\
\hline ER & ML & k3 & 3 & W6014 & $\mathrm{b}$ & MDJ & $1,310 \pm 160$ \\
\hline ES & ML & $(\mathrm{k} 1 \mathrm{o})$ & 2 & W3853 & $\mathrm{a}$ & PWL & $8,550 \pm 100$ \\
\hline ES & $\mathrm{KI}$ & pa2 & 2 & W3859 & $\mathrm{a}$ & PWL & $2,080 \pm 70$ \\
\hline ES & ML & $\mathrm{k} 4$ & 2 & W 3880 & a & JPL & $530 \pm 60$ \\
\hline ES & ML & $\mathrm{k} 4$ & 2 & W4006 & $a$ & PWL & $290 \pm 70$ \\
\hline ES & ML & $\mathrm{k} 4$ & 2 & W4360 & a & JPL & $300 \pm 60$ \\
\hline ES & ML & $(k 4)$ & 2 & W 4367 & $\mathrm{a}$ & RBM & $570 \pm 70$ \\
\hline ES & ML & $(k 1 y)$ & 2 & W4803 & a & JPL & $8,740 \pm 100$ \\
\hline ES & ML & $\mathrm{k} 4$ & 2 & W4805 & $\mathrm{a}$ & JPL & $340 \pm 60$ \\
\hline ES & ML & $\mathrm{k} 4$ & 2 & W 4845 & $\mathrm{a}$ & JPL & $640 \pm 70$ \\
\hline
\end{tabular}


Table 2.-Radiocarbon ages-Continued

\begin{tabular}{|c|c|c|c|c|c|c|c|}
\hline $\begin{array}{l}\text { Quadrangle } \\
\text { code }\end{array}$ & Volcano & $\begin{array}{l}\text { Dated map } \\
\text { unit }\end{array}$ & $\begin{array}{l}\text { Map sheet } \\
\text { No. }\end{array}$ & $\begin{array}{l}\text { Laboratory } \\
\text { No. }\end{array}$ & Remark & Collector & $\begin{array}{c}{ }^{14} \mathrm{C} \text { age (years } \\
\text { B.P.) }\end{array}$ \\
\hline$\overline{E S}$ & $\mathrm{ML}$ & $\mathrm{k} 4$ & 2 & W5106 & $a$ & JPL & $300 \pm 80$ \\
\hline ES & ML & $\mathrm{k} 2$ & 2 & W58 15 & $b$ & JPL & $2,270 \pm 130$ \\
\hline ES & $\mathrm{KI}$ & $(p 4 o)$ & 2 & W6007 & $b$ & JPL & $580 \pm 100$ \\
\hline ES & $\mathrm{KI}$ & pa2 & 2 & GM394 & $e$ & DMD & $2,170 \pm 200$ \\
\hline ET & $\mathrm{KI}$ & $(p 4 o)$ & 2 & W3999 & a & PWL & $730 \pm 80$ \\
\hline ET & $\mathrm{KI}$ & $(\mathrm{p} 2)$ & 2 & W5345 & a & JPL & $2,770 \pm 150$ \\
\hline ET & $\mathrm{KI}$ & $(p 4 o)$ & 2 & W5897 & $b$ & CAN & $550 \pm 150$ \\
\hline EU & $\mathrm{KI}$ & $\mathrm{p} 4$ & 2 & WW 117 & c & FAT & $320 \pm 70$ \\
\hline EU & $\mathrm{KI}$ & $\mathrm{p} 4$ & 2 & WW 117 & c & FAT & $340 \pm 80$ \\
\hline EV & $\mathrm{KI}$ & p 5-1790 & 2 & W4639 & a & RBM & $<200$ \\
\hline EV & $\mathrm{KI}$ & $p c 3$ & 2 & W 4674 & a & $\mathrm{RBM}$ & $1,270 \pm 70$ \\
\hline EV & $\mathrm{KI}$ & p 40 & 2 & W4688 & a & RBM & $490 \pm 60$ \\
\hline EV & $\mathrm{KI}$ & pc3 & 2 & W4690 & a & $\mathrm{RBM}$ & $900 \pm 70$ \\
\hline $\mathrm{EW}$ & $\mathrm{KI}$ & $\mathrm{p} 4 \mathrm{y}$ & 2 & W5048 & a & RBM & $340 \pm 60$ \\
\hline FN & $\mathrm{HU}$ & h2 & 1 & W4388 & a & $\mathrm{RBM}$ & $2,290 \pm 70$ \\
\hline FN & $\mathrm{HU}$ & $\mathrm{h}$ & 1 & W4391 & a & RBM & $10,370 \pm 150$ \\
\hline FN & ML & $\mathrm{k}$ & 1 & W4392 & a & RBM & $13,800 \pm 300$ \\
\hline $\mathrm{FO}$ & ML & k3 & 1 & W5519 & a & RBM & $1,150 \pm 200$ \\
\hline $\mathrm{FO}$ & $\mathrm{HU}$ & h4 & 1 & W5531 & a & RBM & $710 \pm 150$ \\
\hline FO & ML & $\mathrm{k} 4$ & 1 & W5568 & a & JPL & $510 \pm 150$ \\
\hline $\mathrm{FO}$ & ML & $\mathrm{k} 3$ & 1 & W5625 & $b$ & JPL & $1,100 \pm 110$ \\
\hline $\mathrm{FO}$ & ML & $\mathrm{k} 2$ & 1 & W5633 & $b$ & JPL & $1,600 \pm 200$ \\
\hline FO & ML & $\mathrm{k} 2$ & 1 & W6054 & b & RBM & $1,540 \pm 120$ \\
\hline FO & ML & k3 & 1 & W6055 & b & FAT & $1,150 \pm 150$ \\
\hline FP & ML & $\mathrm{k} 1 \mathrm{y}$ & 1 & W5542 & $b$ & JPL & $3,200 \pm 200$ \\
\hline FP & ML & $\mathrm{k} 3$ & 1 & W5818 & $b$ & CDC & $940 \pm 100$ \\
\hline FP & ML & $\mathrm{k} 3$ & 1 & W5829 & $b$ & $\mathrm{CDC}$ & $1,020 \pm 100$ \\
\hline FR & ML & $\mathrm{k} 4$ & 1 & W4 118 & a & JPL & $580 \pm 80$ \\
\hline FR & ML & $\mathrm{k} 4$ & 1 & W4 175 & a & JPL & $300 \pm 50$ \\
\hline FR & ML & $\mathrm{k} 4$ & 1 & W4576 & a & JPL & $480 \pm 60$ \\
\hline FR & ML & $\mathrm{k} 2$ & 1 & W4579 & $\mathrm{a}$ & JPL & $2,120 \pm 70$ \\
\hline FR & ML & $\mathrm{k} 4$ & 1 & W4916 & a & JPL & $560 \pm 70$ \\
\hline FS & ML & $\mathrm{k} 4$ & 2 & W3790 & a & JPL & $420 \pm 70$ \\
\hline FS & ML & $\mathrm{k} 4$ & 2 & W3793 & a & JPL & $450 \pm 60$ \\
\hline FS & ML & k3 & 2 & W4237 & a & JPL & $1,320 \pm 50$ \\
\hline FS & ML & $\mathrm{k} 4$ & 2 & W4338 & a & JPL & $590 \pm 50$ \\
\hline FS & ML & $\mathrm{k} 3$ & 2 & W4340 & a & JPL & $1,400 \pm 70$ \\
\hline FS & $\mathrm{ML}$ & $\mathrm{k} 4$ & 2 & W4341 & $\mathrm{a}$ & JPL & $330 \pm 60$ \\
\hline FS & ML & $\mathrm{k} 2$ & 2 & W4 344 & a & JPL & $1,690 \pm 70$ \\
\hline FS & $\mathrm{ML}$ & k3 & 2 & W4357 & a & JPL & $1,490 \pm 50$ \\
\hline FS & $\mathrm{ML}$ & $\mathrm{k} 1 \mathrm{y}$ & 2 & W4358 & a & JPL & $4,070 \pm 70$ \\
\hline FS & ML & $\mathrm{k} 3$ & 2 & W 4794 & a & JPL & $880 \pm 60$ \\
\hline FS & $\mathrm{ML}$ & $\mathrm{k} 2$ & 2 & W4810 & $\mathrm{a}$ & JPL & $2,550 \pm 80$ \\
\hline FS & $\mathrm{ML}$ & $\mathrm{k} 2$ & 2 & W4985 & a & JPL & $1,760 \pm 60$ \\
\hline FS & $\mathrm{ML}$ & $\mathrm{k} 1 \mathrm{o}$ & 2 & W4991 & a & JPL & $7,960 \pm 110$ \\
\hline FS & $M L$ & $\mathrm{k}$ & 2 & W4995 & $\mathrm{a}$ & JPL & $10,400 \pm 150$ \\
\hline FT & KI & $\mathrm{p} 4$ & 2 & W5631 & $b$ & JPL & $580 \pm 200$ \\
\hline FT & $\mathrm{ML}$ & $\mathrm{k}$ & 2 & W5942 & $\mathrm{b}$ & JPL & $10,660 \pm 400$ \\
\hline FU & ML & $\mathrm{k} 1 \mathrm{o}$ & 2 & W3862 & a & PWL & $5,650 \pm 90$ \\
\hline FU & $\mathrm{KI}$ & $\mathrm{p} 4$ & 2 & W 3881 & a & PWL & $260 \pm 70$ \\
\hline FU & $\mathrm{KI}$ & p 4 & 2 & W3941 & a & $\mathrm{RTH}$ & $450 \pm 60$ \\
\hline $\mathrm{FU}$ & ML & $\mathrm{k} 10$ & 2 & W4536 & $\mathrm{a}$ & JPL & $5,250 \pm 100$ \\
\hline $\mathrm{FU}$ & $\mathrm{KI}$ & p 4 & 2 & W4661 & a & $\mathrm{RBM}$ & $320 \pm 70$ \\
\hline GN & $\mathrm{HU}$ & h1y & 1 & W4376 & a & $\mathrm{RBM}$ & $3,600 \pm 70$ \\
\hline GN & $\mathrm{HU}$ & hc $1 y$ & 1 & W4378 & a & RBM & $4,720 \pm 80$ \\
\hline GN & $\mathrm{HU}$ & h5-1800-01 & 1 & W4634 & $\mathrm{a}$ & $\mathrm{RBM}$ & $<200$ \\
\hline GN & HU & $\mathrm{h}$ & 1 & W5056 & a & RBM & $12,950 \pm 150$ \\
\hline
\end{tabular}


Table 2.-Radiocarbon ages-Continued

\begin{tabular}{|c|c|c|c|c|c|c|c|}
\hline $\begin{array}{l}\text { Quadrangle } \\
\text { coxle }\end{array}$ & Volcano & $\begin{array}{l}\text { Dated map } \\
\text { unit }\end{array}$ & $\begin{array}{l}\text { Map sheet } \\
\text { No. }\end{array}$ & $\begin{array}{l}\text { Laboratory } \\
\text { No. }\end{array}$ & Remark & Collector & $\begin{array}{c}{ }^{14} \mathrm{C} \text { age (years } \\
\text { B.P.) }\end{array}$ \\
\hline $\mathrm{GN}$ & $\mathrm{HU}$ & h2 & 1 & W5068 & $\bar{a}$ & $\mathrm{RBM}+\mathrm{DAC}$ & $2,350 \pm 80$ \\
\hline GN & HU & h2 & 1 & W5068 & a & $\mathrm{RBM}+\mathrm{DAC}$ & $2,350 \pm 80$ \\
\hline GN & $\mathrm{HU}$ & h5-1800-01 & 1 & W5124 & a & DAC & $<200$ \\
\hline GN & $\mathrm{HU}$ & h1y & 1 & W5127 & a & $\mathrm{RBM}+\mathrm{DAC}$ & $3,100 \pm 80$ \\
\hline GN & HU & h3 & 1 & W5130 & a & $\mathrm{RBM}+\mathrm{DAC}$ & $1,030 \pm 60$ \\
\hline GN & $\mathrm{HU}$ & h2 & 1 & W5788 & b & $R B M+D A C$ & $1,590 \pm 100$ \\
\hline GN & $\mathrm{HU}$ & h1y & 1 & W5789 & $b$ & $\mathrm{RBM}+\mathrm{DAC}$ & $4,700 \pm 350$ \\
\hline GN & $\mathrm{HU}$ & h2 & 1 & W5796 & $\mathrm{b}$ & RBM & $2,700 \pm 100$ \\
\hline GN & $\mathrm{HU}$ & h1y & 1 & W5801 & $\mathrm{b}$ & RBM & $3,020 \pm 150$ \\
\hline GN & $\mathrm{HU}$ & h2 & 1 & W5828 & b & RBM & $2,140 \pm 100$ \\
\hline GN & HU & h1o & 1 & W5978 & $b$ & RBM & $7,520 \pm 200$ \\
\hline GN & $\mathrm{HU}$ & h1o & 1 & W5981 & $b$ & RBM & $6,260 \pm 180$ \\
\hline GN & $\mathrm{HU}$ & h1o & 1 & AA4 387 & c & RBM & $7,000 \pm 95$ \\
\hline GN & $\mathrm{HU}$ & h2 & 1 & AA 4388 & c & RBM & $2,740 \pm 100$ \\
\hline GN & $\mathrm{HU}$ & h2 & & 1 & RD & $d$ & $\mathrm{RD}$ \\
\hline GO & HU & hc 2 & 1 & W5073 & a & $\mathrm{RBM}+\mathrm{DAC}$ & $2,390 \pm 60$ \\
\hline GO & $\mathrm{HU}$ & h2 & 1 & W5076 & $\mathrm{a}$ & $\mathrm{RBM}+\mathrm{DAC}$ & $2,670 \pm 80$ \\
\hline GO & $\mathrm{HU}$ & h1y & 1 & W5132 & a & RBM & $3,990 \pm 70$ \\
\hline GO & $\mathrm{HU}$ & h1o & 1 & W5297 & a & $\mathrm{RBM}+\mathrm{DAC}$ & $6,360 \pm 100$ \\
\hline GO & $\mathrm{HU}$ & he 10 & 1 & W5299 & a & $\mathrm{RBM}+\mathrm{DAC}$ & $8,770 \pm 200$ \\
\hline GO & HU & h3 & 1 & W5491 & a & RBM & $900 \pm 110$ \\
\hline GO & $\mathrm{HU}$ & h3 & 1 & W5522 & $a$ & RBM & $1,180 \pm 200$ \\
\hline GP & ML & k3 & 1 & W5809 & b & EWW & $1,500 \pm 100$ \\
\hline GP & ML & k3 & 1 & AZ2 & c & JPL & $1,267 \pm 100$ \\
\hline GQ & $M L$ & $\mathrm{k} 4$ & 1 & W4 186 & $\mathrm{a}$ & JPL & $220 \pm 60$ \\
\hline GQ & ML & $\mathrm{k} 4$ & 1 & W 4537 & a & JPL & $270 \pm 60$ \\
\hline $\mathrm{GQ}$ & ML & $\mathrm{k} 4$ & 1 & W5792 & $b$ & JPL & $1,500 \pm 150$ \\
\hline $\mathrm{GQ}$ & ML & k3 & 1 & W5797 & b & JPL & $1,570 \pm 100$ \\
\hline GQ & $M L$ & k4 & 1 & W5803 & b & JPL & $390 \pm 100$ \\
\hline $\mathrm{GQ}$ & ML & k4 & 1 & W5976 & $\mathrm{b}$ & JPL & $330 \pm 100$ \\
\hline GQ & ML & k3 & 1 & W5989 & $\mathrm{b}$ & JPL & $1,500 \pm 130$ \\
\hline $\mathrm{GQ}$ & ML & $\mathrm{k} 2$ & 1 & $\mathrm{AZ} 1$ & c & JPL & $1,891 \pm 99$ \\
\hline GR & ML & $\mathrm{k} 4$ & 1 & W5079 & $\mathrm{a}$ & JPL & $410 \pm 60$ \\
\hline GR & MK & ly & 1 & W5541 & $\mathrm{a}$ & JPL & $5,630 \pm 200$ \\
\hline GR & $\mathrm{ML}$ & $\mathrm{k} 2$ & 1 & W5566 & a & JPL & $2,240 \pm 150$ \\
\hline GR & MK & ly & 1 & W5773 & $b$ & EWW & $5,320 \pm 150$ \\
\hline GR & $\mathrm{MK}$ & ly & 1 & W5776 & b & EWW & $4,510 \pm 150$ \\
\hline GR & MK & ly & 1 & W5779 & b & EWW & $4,550 \pm 150$ \\
\hline GR & ML & $\mathrm{k} 2$ & 1 & W5949 & $\mathrm{b}$ & JPL & $1,880 \pm 200$ \\
\hline GR & ML & $k 2$ & 1 & W5985 & $b$ & JPL & $1,580 \pm 120$ \\
\hline GS & ML & $\mathrm{k}$ & 2 & W5092 & $\mathrm{a}$ & JPL & $10,090 \pm 120$ \\
\hline GS & $M L$ & $k 1 y$ & 2 & W5112 & $\mathrm{a}$ & JPL & $3,110 \pm 60$ \\
\hline GS & ML & $\mathrm{k} 1 \mathrm{y}$ & 2 & W5565 & a & JPL & $3,790 \pm 200$ \\
\hline GS & ML & $k 1 y$ & 2 & W5567 & $a$ & JPL & $4,790 \pm 250$ \\
\hline GT & ML & $\mathrm{k} 2$ & 2 & W 477 & $\mathrm{a}$ & GAM & $2,000 \pm 250$ \\
\hline GT & $M L$ & $\mathrm{k} 2$ & 2 & W478 & $\mathrm{a}$ & GAM & $2,070 \pm 250$ \\
\hline GT & ML & $k 10$ & 2 & W 4529 & a & JPL & $9,540 \pm 110$ \\
\hline GT & ML & $\mathrm{k} 1 \mathrm{y}$ & 2 & W 4574 & a & JPL & $3,700 \pm 70$ \\
\hline GT & ML & $k 1 y$ & 2 & W4624 & $a$ & JBB & $3,380 \pm 80$ \\
\hline GT & ML & $k 1 y$ & 2 & W4957 & a & JPL & $4,170 \pm 90$ \\
\hline GT & ML & k1o & 2 & W4969 & $\mathrm{a}$ & JPL & $8,030 \pm 70$ \\
\hline GT & ML & $\mathrm{k} 1 \mathrm{o}$ & 2 & W5087 & a & JPL & $9,960 \pm 70$ \\
\hline GT & ML & k & 2 & W5091 & a & JPL & $14,080 \pm 160$ \\
\hline GT & ML & k & 2 & W5094 & a & JPL & $28,120 \pm 400$ \\
\hline GT & ML & $\mathrm{k} 4$ & 2 & W5098 & a & JPL & $570 \pm 60$ \\
\hline GT & ML & $k 1 y$ & 2 & W5107 & a & JPL & $3,450 \pm 60$ \\
\hline GT & ML & $\mathrm{k}$ & 2 & W5452 & a & JPL & $28,200 \pm 600$ \\
\hline
\end{tabular}


Table 2.-Radiocarbon ages-Continued

\begin{tabular}{|c|c|c|c|c|c|c|c|}
\hline $\begin{array}{l}\text { Quadrangle } \\
\text { code }\end{array}$ & Volcano & $\begin{array}{l}\text { Dated map } \\
\text { unit }\end{array}$ & $\begin{array}{l}\text { Map sheet } \\
\text { No. }\end{array}$ & $\begin{array}{l}\text { Laboratory } \\
\text { No. }\end{array}$ & Remark & Collector & $\begin{array}{c}{ }^{14} \mathrm{C} \text { age (years } \\
\text { B.P.) }\end{array}$ \\
\hline GT & $\mathrm{ML}$ & k & 2 & W6062 & b & JPL & $14,240 \pm 400$ \\
\hline GU & ML & k3 & 2 & W4343 & a & JPL & $1,280 \pm 70$ \\
\hline GU & ML & $\mathrm{k}$ & 2 & W4620 & a & JBB & $14,500 \pm 200$ \\
\hline GU & $M L$ & k & 2 & W4623 & a & JBB & $10,610 \pm 150$ \\
\hline GU & $\mathrm{ML}$ & $\mathrm{k}$ & 2 & W4627 & a & JBB & $13,530 \pm 180$ \\
\hline GU & ML & $k 1 y$ & 2 & W4629 & a & JBB & $3,360 \pm 80$ \\
\hline GU & ML & $\mathrm{k} 3$ & 2 & W4631 & a & JBB & $1,110 \pm 60$ \\
\hline GU & ML & $k 10$ & 2 & W 4884 & a & JBB & $9,780 \pm 140$ \\
\hline GU & ML & $\mathrm{k}$ & 2 & W4971 & a & JBB & $14,080 \pm 150$ \\
\hline GU & $M L$ & $\mathrm{k}$ & 2 & W4973 & a & JBB & $14,370 \pm 190$ \\
\hline GU & ML & k & 2 & W4977 & a & JBB & $14,530 \pm 120$ \\
\hline GU & $M L$ & k3 & 2 & W4981 & $\mathbf{a}$ & JPL & $1,470 \pm 50$ \\
\hline GU & ML & $k 1 y$ & 2 & W5077 & a & JBB & $4,410 \pm 120$ \\
\hline GU & ML & k3 & 2 & W5597 & a & JBB & $1,140 \pm 300$ \\
\hline $\mathrm{HN}$ & HU & h2 & 1 & W5559 & a & $\mathrm{DAC}$ & $3,030 \pm 200$ \\
\hline $\mathrm{HO}$ & HU & h2 & 1 & W4171 & a & RBM & $2,030 \pm 80$ \\
\hline $\mathrm{HO}$ & HU & $\mathrm{h}$ & 1 & W4365 & a & RBM & $12,230 \pm 150$ \\
\hline $\mathrm{HO}$ & HU & h1o & 1 & $W 4371$ & a & RBM & $9,490 \pm 100$ \\
\hline $\mathrm{HO}$ & ML & $k 1 y$ & 1 & W5536 & a & JPL & $3,360 \pm 200$ \\
\hline $\mathrm{HO}$ & $\mathrm{HU}$ & hly & 1 & W5562 & a & DAC & $3,610 \pm 200$ \\
\hline $\mathrm{HQ}$ & ML & $\mathrm{k} 4$ & 1 & W5772 & b & EWW & $300 \pm 100$ \\
\hline HS & MK & ly & 2 & W5823 & b & WSW & $4,850 \pm 150$ \\
\hline IR & MK & ly & 1 & W5821 & b & MAK & $7,100 \pm 150$ \\
\hline IR & MK & ly & 1 & W5825 & b & MAK & $4,410 \pm 150$ \\
\hline IS & MK & ly & 2 & AA6795 & c & PMV & $5,085 \pm 97$ \\
\hline
\end{tabular}

\section{REFERENCES CITED}

Cross, C.W., 1904, An occurrence of trachyte on the Island of Hawaii: Journal of Geology, v. 12, p. 510-523.

Dorn, R.I., Jull, A.T.J., Donahue, D.J., Linick, T.W., Toolin, L.J., Moore, R.B., Rubin, M., Gill, T.E., and Cahill, T.A., 1992, Rock varnish on Hualalai and Mauna Kea volcanoes, Hawaii: Pacific Science, v. 46, no. 1 , p. 11-34.

Jackson, M.D., Endo, E.T., Delaney, P.T., Arnadottir, Thora, and Rubin, A.M., 1992, Ground ruptures of the 1974 and 1983 Kaoiki earthquakes Mauna Loa Volcano, Hawaii: Journal of Geophysical Research, v. 97, no. B6, p. 8,775-8,796.

Mueller-Dombois, Dieter, and Lamoreux, C.H., 1967, Soil-vegetation relationships in Hawaiian kipukas: Pacific Science, v. 21, p. 286-289.
Rubin, Meyer, Gargulinski, L.K., and McGeehin, J.P., 1987, Hawaiian radiocarbon dates, in Decker, R.W., Wright, T.L., and Stauffer, P.H., eds., Volcanism in Hawaii: U.S. Geological Survey Professional Paper 1350, v. 1, p. 213-242.

Shapiro, Leonard, 1975, Rapid analysis of silicate, carbonate, and phosphate rocks-revised edition: U.S. Geological Survey Bulletin 1401, 76 p.

Washington, H.S., 1923, Petrology of the Hawaiian Islands: American Journal of Science, 5th series, v. 6, p. 100-126.

Wolfe, E.W., 1996, Chemical analyses for the geologic map of the Island of Hawaii: U.S. Geological Survey Open-File Report OF-96-504. 
